\section{P3-403 Thyroid}

Vasculitis as Complication in Two Children Receiving Propylthiouracyl for Graves' Disease

C. Jeandel*1; A. Kucharska*2; K. Grela*2;

B. Rymkiewicz-Kluczynska ${ }^{2}$; J. Astruc ${ }^{* 1} ;$ R. Dumas ${ }^{* 1} ;$ Ch. Sultan ${ }^{1}$

1 Unité d’Endocrinologie-Gynécologie Pédiatriques, Service de

Pédiatrie 1, Hôpital A. de Villeneuve, Montpellier, France; ${ }^{2}$ Pediatrics

Endocrinology, Medical Department, University of Warsaw, Poland

In adults, the use of propylthiouracyl (PTU) in patients with Graves' disease has been associated with multiple complications including rash, leucocytoclastic vasculitis, pulmonary hemorrhage, glomerulonephritis, and the presence of perinuclear antineutrophilic cytoplasmic antibodies (pANCA). We report 2 children (1 from France and 1 from Poland) who developed vasculitis during PTU treatment for Graves' disease. Case 1: Eleven-year-old girl, with Graves' disease treated for 3 years with PTU. Successively occurred fever with polyarthralgia and thinning, autoimmune hemolytic anemia, glomerulonephritis (HSF) and multiple pulmonary excavations. She presented bilateral sinusitis and rhinitis. Her serum was positive for homogeneous nuclear antibodies (1/ $500 \mathrm{e})$, native anti-DNA antibodies $(61 \mathrm{UI} / \mathrm{ml})$ and perinuclear anti neutrophilic cytoplasmic antibodies (pANCA 1/8000e). The diagnosis of Wegener's granulomatosis was settled. She required cyclophosphamid and systemic corticotherapy. Graves' disease was well controlled and PTU was stopped. All clinical manifestations of vasculitis disappeared but biological anomalies of autoimmunity remained (ANA, pANCA, native anti DNA antibodies). Case 2: Forteen-year-old girl with Down syndrome treated for 13 months with PTU for Graves' disease. She developped consecutively peritonitis, pericarditis and pleuritis. SLE diagnosis was established with positive antinuclear and anti neutrophil cells antibodies (ANA: 1/160, pANCA 1/80). PTU treatment was stopped and glucocorticoid therapy was started. After 6 weeks of treatment hyperthyroidism reappeared and radioiodine treatment was prescribed. Conclusion: Although the relationship between Graves' disease, PTU treatment and vasculitis hasn't yet been clearly understood, these two privileged case reports point out the usefulness of clinical and biological follow-up of patients with Graves' disease treated with PTU for their risk of developing vasculitis, SLE or Wegener's granulomatosis.

\section{P3-404 Thyroid}

\section{Neonatal Hyperthyroidism in Twins following Maternal Thyroidectomy}

V. Borras*1; P. Gussinyé*1; M. Peñas*1; A. Zuasnabar*1;

M. Català ${ }^{*}$; Introduced by L. Castaño

${ }^{1}$ Hospital General de Granollers, Barcelona, Spain

Introduction: Neonatal hyperthyroidism is a relatively rare condition usually produced by the transplacental passage of thyroid-stimulating immunoglobulins. Most commonly, this antibodies are a components of active maternal Graves' disease. However, such antibodies may still be produced after ablation of the thyroid through surgery, radioiodine, or other inmunologic mechanisms. Case Reports: Twins were born after 33 weeks of gestation to a mother with Graves' disease. The mother had undergone a subtotal thyroidectomy one month before becoming pregnant. Both twins showed hyperthyroidism symptoms at the first week of life (tachycardia, poor weight gain, exophthalmos, hyperkinesis). Their initial thyroid function tests were: $\mathrm{T}_{4} \mathrm{~L}>6 \mathrm{ng} / \mathrm{dl}(0.7-1.87)$ TSI $>95(<10)$ and TSH male: $<0.03$ and female: $0.34 \mu \mathrm{U} / \mathrm{ml}(0.25-4.67)$. Hormone analysis and treatment evolution are shown in the following figure:
Commentary: Transfer of thyroid-stimulating immunoglubulins to the fetus during pregnancy is possible even in mothers who have undergone a subtotal thyroidectomy as a permanent treatment of their hyperthyroidism.

\section{P3-405 Thyroid \\ Graves' Disease (GD) in Children and Adolescents - Critical Evaluation of the Therapeutic \\ S. Nesi-França*1; M.P. Khaled*1; C. Albino*2; R. Sandrini ${ }^{3}$. \\ L. De Lacerda*4; H. Graf*1; Introduced by M.C.S. Boguszewski \\ Curitiba, Brazil; ${ }^{2}$ Estadual University of Maring, Maring, Brazil; \\ ${ }^{3}$ Paediatric Endocrinology Unit, Curitiba, Brazil; ${ }^{4}$ Curitiba, Brazil}

GD is the most common cause of thyrotoxicosis in children and adolescents, characterised by diffuse goiter, hyperthyroidism and ophthalmopathy. There is no specific cure for the illness, and potential complications are associated with each therapeutic option (antithyroid drugs (ATD), ${ }^{131} \mathrm{I}$ and surgery). Our aims in this study were the clinical, laboratory and therapeutic evaluation of 65 children and adolescents with GD, followed in two endocrinology units, from 1988 to 1998 . The results found in our study were: 65 patients (52 females and 13 males) were evaluated, 18 prepubertal and 47 pubertal. The average age was $12.6 \pm 4$ years (range from 2.7 to 18 years) and $44.6 \%(29 / 65)$ had familiar thyroid disease. All the patients had behavioural disturbances (irritability, poor academic performance, hyperactivity) and diffuse goiter (average $50 \mathrm{~g}$ ); 30/65 had weight loss, $36 / 65$ increase of appetite and 44/65 ophthalmopathy (mild to moderate). The initial mean values of T3 $(\mathrm{ng} / \mathrm{dl}), \mathrm{T} 4(\mu \mathrm{g} / \mathrm{dl})$ and ${ }^{131} \mathrm{I}$ uptake $(24 \mathrm{~h}, \%)$ were: $505.6 \pm 205.7(160.0-1230.0), 21.9 \pm 6.6(10.0-40.7)$ and $59.9 \pm 16.8(8.9-87.4)$, respectively, with the mean laboratory values slightly higher in prepubertal patients. Sixty-three patients received ATD (28 propylthiouracil-PTU and 35 methimazole-MTZ), with posterior addition of levothyroxine in 44/63, two patients were submitted to surgery as first choice. The side effects of the ATD, specially PTU, were present in 19\%(12/63) of the patients, such as agranulocitosis, arthritis, arthralgia, rash, purpura, vasculitis, lupus-like syndrome, hepatitis and purpura fulminans. Twenty-nine patients that received ATD were then submitted to ${ }^{131} \mathrm{I}$, from which 23 developed hypothyroidism and 4 patients were submitted to surgery, two of them developed permanent hypoparathyroidism. Our data indicate that the therapy with ATD, specially PTU, is associated with a high incidence of side effects and that the therapy with radioiodine, although associated to a high incidence of hypothyroidism, is a safe therapy in children and adolescents.

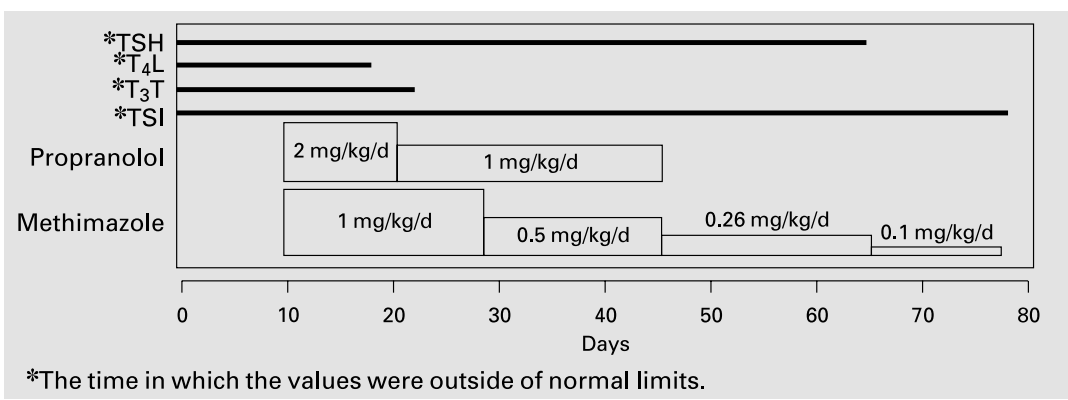




\section{P3-406 Thyroid}

Longitudinal Study of Type 1 Diabetes (T1D) Risk Markers in PTS with Autoimmune Thyroid Disease (ATD)

D. Larizza ${ }^{1} ;$ M. Locatelli*2; C. Calcaterra*1; M. Martinetti*3.

E. Lenta* ${ }^{1} ;$ G. d'Annunzio*1; R. Lorini ${ }^{4}$

${ }^{1}$ Dip. di Scienze Pediatriche, Univ. di Pavia; ${ }^{2}$ Direzione Scientifica, Ospedale Pediatrico Bambino Gesù, Roma; ${ }^{3}$ Servizio di Immunoematologia e Trasfusione IRCCS Policlinico S. Matteo, Pavia; ${ }^{4}$ Dip. di Pediatria, Univ. di Genova, IRCCS G. Gaslini, Genova, Italy

Patients with ATD are at risk of developing other autoimmune diseases, such as T1D. This study is aimed to evaluate prospectively the risk for T1D conferred by immunological and genetic markers, in a cohort of 90 patients with ADT (77 females and 13 males, median age 13.8 yrs, range 4.3-25.5). Among these patients, 68 are affected by autoimmune thyroiditis (AT) and 22 by Graves' disease (GD). At the time of recruitment, first phase insulin response (FPIR) to an intravenous glucose tolerance test, protein tyrosine phosphatase (IA2A), glutamic acid decarboxylase (GADA) and islet cell antibodies (ICA) were determined. HLA-DQ $\alpha$ and $\mathrm{DQ} \beta$ typing was also performed in 87 of them. All patients were followed up for $2.8 \pm 1.2 \mathrm{yrs}$, and a second evaluation of FPIR, GADA and IA2A has been repeated in 54 patients. None of the patients of this cohort developed TID. On the basis of HLA-DQ $\alpha$ and DQ $\beta$ genotypes, the patients were divided in: reduced (R), low $(\mathrm{L})$, moderate $(\mathrm{M})$ and high $(\mathrm{H})$ risk for T1D. A summary of the results is reported in the following table.

\begin{tabular}{|c|c|c|c|c|}
\hline & $\begin{array}{l}\text { ICA } \\
\text { (+/all) }\end{array}$ & $\begin{array}{l}\text { GADA } \\
\text { (+/all) }\end{array}$ & $\begin{array}{l}\text { IA2A } \\
\text { (+/all) }\end{array}$ & $\begin{array}{l}\text { FPIR } \\
(<1 \text { st cent/all) }\end{array}$ \\
\hline I evaluat. (90 patients) & $5 / 60$ & $1 / 86$ & $1 / 76$ & $2 / 90$ \\
\hline $\mathrm{R}$ risk $\mathrm{n}=40$ & 2 & 1 & 0 & 1 \\
\hline $\mathrm{L}$ risk $\mathrm{n}=46$ & 3 & 0 & 1 & 1 \\
\hline $\mathrm{M}$ risk $\mathrm{n}=1$ & 0 & 0 & 0 & 0 \\
\hline $\mathrm{H}$ risk $\mathrm{n}=0$ & - & - & - & - \\
\hline Risk unknown $\mathrm{n}=3$ & 0 & 0 & 0 & 0 \\
\hline Follow-up. (54 patients) & n.d. & $2 / 46$ & $1 / 44$ & $1 / 54$ \\
\hline $\mathrm{R}$ risk $\mathrm{n}=25$ & & 2 & 0 & 0 \\
\hline L risk $n=29$ & & 0 & 1 & 1 \\
\hline
\end{tabular}

The prevalence of islet-related autoantibodies in ATD patients is similar to that found in general population. Moreover the frequency of protective genes was higher in our patients than in general population, while the frequency of susceptible ones was similar. On the basis of our data, patients with ATD seem to have low risk of developing T1D.

\section{P3-407 Thyroid}

Transient Congenital Hypothyroidism (TCH): Characteristics and Estimated Incidence

C. Mengreli ${ }^{1}$;H. Tsokas ${ }^{2}$; A. Vagenakis ${ }^{* 3}$; C. Dacou-Voutetakis ${ }^{2}$

${ }^{1}$ Inst. of Child Health, ${ }^{2}$ Aghia Sophia Childrens Hospital, Athens;

${ }^{3}$ Medical Univ., Patras, Greece

Neonatal screening programme for $\mathrm{CH}$ has revealed also cases with $\mathrm{TCH}$ persisting for a variable period after birth and requiring thyroxine (T4) treatment. The pathogenetic mechanism varies. In some cases it is present in newborns of women with autoimmune thyroid disease. We present data of TCH in 9 children, $(5 \mathrm{M}, 4 \mathrm{~F})$, who were detected after screening 1,310,071 newborns born to mothers with Hashimoto thyroditis. Mean serum values of T4 were $5.1 \mu \mathrm{g} / \mathrm{dl}$ (r.r. 6-16) and TSH $444 \mathrm{mU} / 1$ (r.r. 0.3-5). TSH receptor-blocking Abs (TRAB) were found in 6 of 6 newborns checked for, whereas the antithyroid Abs (anti-TPO and/or anti-Tg) were present in 8 cases. T4 replacement therapy was given from a mean age of 15 days till the first year of life. There was no need for T4 dose adaptation during this period. Thyroid echogram and/or scintiscan in 4 of these children showed a normal thyroid gland. Serial measurements of serum levels of Abs revealed disappearance of TRAB by the 4 th month of life and of the anti-TPO and anti-Tg between the 3rd and 9th month depending on the initial concentration. On the basis of our data we conclude that the incidence of TCH is $1: 145,563$ newborns and constitutes $2.3 \%$ of newborns with $\mathrm{CH}$. A rather characteristic finding is low $\mathrm{T} 4$ requirement with advancinq age. Maternal autoimmune thyroid disease should raise the question for $\mathrm{TCH}$ in the offspring.

39th Annual Meeting of the ESPE

\section{P3-408 Thyroid}

Relation of Etiology to Treatment in Children with Congenital Hypothyroidism

A. Hanukoglu*1; K. Perlman*2; I. Shamis*2; L. Brnjac*2; J. Rovet*2; D. Daneman*2 ${ }^{*}$ Introduced by $A$. Rosler

${ }^{1} \mathrm{E}$. Wolfson Hospital, Holon, Israel; ${ }^{2}$ University of Toronto, Toronto, Canada

Severity of congenital hypothyroidism $(\mathrm{CH})$, determined by the degree of skeletal delay or hormonal derangement, may effect the outcome of $\mathrm{CH}$. These factors may also be related to etiology of $\mathrm{CH}$. Objective: To examine whether the pattern of TSH, T4 concentrations, and treatment schedules should differ by etiology determined by radionuclide imaging studies. Patients and Methods: We evaluated 125 children $(50 \mathrm{M}, 75 \mathrm{~F})$ with $\mathrm{CH}$ from diagnosis to 4 years of age who were divided into three groups: (i) Athyrotic $\mathrm{CH}$ : $\mathrm{N}=31$; (ii) Dysgenetic $\mathrm{CH}: \mathrm{N}=54$; (iii) Dyshormonogenetic $\mathrm{CH}: \mathrm{N}=40$. Follow-up evaluation was carried out at 2-4 wk, and 3, 6, 9, 12, 24, 36, 48 months of age. Results: The median gestational age (40 wk), age at onset of therapy (12-13 days) and starting L-T4 dose $(9.2-9.4 \mu \mathrm{g} / \mathrm{kg})$ were similar in all three groups. The median screening TSH levels were significantly higher $(\mathrm{p}<0.02)$ and confirmatory $\mathrm{T} 4$ lower in infants with athyreosis than the other two groups $(\mathrm{p}<0.01$ vs. dysgenetic, $\mathrm{p}<0.05$ vs. dyshormonogenetic $\mathrm{CH})$. During the first 6 months of therapy mean TSH levels remained higher in the athyrotic group than the other two groups. At 12 months dysgenetic patients had the highest TSH. During the entire study period in patients with dyshormonogenesis, the mean TSH levels remained lowest and normalized earlier $(9 \mathrm{mo})$. TSH elevations in different groups and ages, corresponded with the number and percentage of patients who required a dose increase in the same group. Mean T4 levels normalized rapidly and remained so throughout the study in all groups. The athyrotic group received the highest and dyshormonogenetic group the lowest dose. Conclusion: Treatment and follow-up schedules for $\mathrm{CH}$ have to consider different hormonal patterns and responses to therapy in the three etiologic categories. While the need for close supervision especially in children with athyreosis is crucial particularly early in life, those with dysgenesis and dyshormonogenesis require more attention later-on.

P1-409 Bone, Ca, PTH and Vitamin D

Development of Bone Density of Spine in Children Aged 3 to 6 Years - A Prospective Study with Dual-Energy X-Ray Absorptiometry

P. Arikoski*1; J. Komulainen ${ }^{1} ;$ R. Voutilainen ${ }^{1}$; H. Kröger ${ }^{* 2}$; Introduced by J. Komulainen

Departments of ${ }^{1}$ Pediatrics and ${ }^{2}$ Surgery, Kuopio University Hospital, Kuopio, Finland

Normative data of bone mineral density (BMD) accretion in young children are scarce. Objective: To evaluate prospectively the development of spinal BMD in healthy growing children aged 3 to 6 years. Patients and Methods: Lumbar spine (L2-L4) BMDareal (g/cm²) was measured by dual-energy x-ray absorptiometry (DEXA) in twenty children (males $n=10$ ) aged 3.3-6.9 (median 4.8) years at baseline and after a median follow-up of 1.0 year (range $0.8-1.1$ years). Apparent volumetric BMD (BMDvol, $\mathrm{g} / \mathrm{cm}^{3}$ ) was calculated to minimize the effect of bone size on BMD in growing spine. Results: During the follow-up, height, weight, lumbar BMDareal and BMDvol increased significantly (table). No significant difference in BMDareal and BMDvol was observed between males and females.

\begin{tabular}{lcc}
\hline & Baseline & 1-year follow-up \\
\hline Height $(\mathrm{cm})$ & $110.6(7.6)$ & $116.8(7.6)^{* *}$ \\
Weight $(\mathrm{kg})$ & $19.8(4.3)$ & $22.6(4.6)^{* *}$ \\
Lumbar BMDareal $\left(\mathrm{g} / \mathrm{cm}^{2}\right)$ & $0.621(0.082)$ & $0.658(0.092)^{* * *}$ \\
Lumbar BMDvol $\left(\mathrm{g} / \mathrm{cm}^{3}\right)$ & $0.262(0.034)$ & $0.271(0.038)^{*}$ \\
\%-change in BMDareal & & $6.0(4.3) \% * * *$ \\
\%-change in BMDvol & & $3.3(3.8) \% *$ \\
\hline
\end{tabular}

$* \mathrm{p}<0.01, * * \mathrm{p} \leq 0.01, * * * \mathrm{p}=0.0001$ vs. baseline (Wilcoxon test), values as mean (SD).

Conclusions: A significant increase in both areal and apparent volumetric BMD was observed in children aged 3 to 6 years during a follow-up of 1 year. The increase in volumetric BMD indicated that there was a real accretion of BMD in growing spine measured by DEXA. 
P1-410 Bone, Ca, PTH and Vitamin D

Contribution of the Vitamin-D Receptor, Collagen lal and Estrogen Receptor Gene Polymorphisms to Bone Mineral Density in Children and Adolescents with Bone Tumors

A. Patiño*1; E. Ruza*1; L. Sierrasesumaga*1;E. Sotillo*1;

M. Zalacain*1; C. Azcona ${ }^{1}$

1 Pamplona, Spain

Bone mineral density (BMD) is a phenotypic trait codified by a number of genes that individually contribute to the final phenotype. It has been demonstrated that genetic polymorphisms in the vitamin D receptor (VDR), estrogen receptor (ER) and collagen IaI (Col IaI) genes may codify both BMD and growth or final height. Objectives: 1 . To characterise the polymorphisms in the above genes in a cohort of paediatric patients with bone tumours and to compare them with a control group sex and age matched. 2. To relate the genotypes detected with the BMD. 3. To evaluate the effect of the chemotherapy in BMD. Materials and Methods: DNA was obtained from 111 paediatric patients with bone tumours (63 osteosarcomas (OS) and 48 Ewings Sarcomas (ES)) and from 88 healthy children. The following polymorphisms were analysed by restriction analysis: VDR gene: Fok I, Apa I and Taq I, Col IaI: Msc I and ER: Pvu II and Xba I. Absence of restriction was represented by uppercase and presence with lower case. BMD measurement was performed in 35 patients with bone tumours by DEXA (Dual-Energy-X ray Absorptiometry, HOLOGIC QDR 4500 Elite, Hologic, Inc, Wolthman, MA). Results: The genotype distribution and allele frequencies were similar in both the group of patients with cancer and the control group. In the group of patients with OS a statistically higher frequency of heterozygotes Ff (VDR gene) $(61.9 \%)$ was observed in comparison to the control group $(43.4 \%)(p=0.026)$. BMD in children with cancer, who have undergone chemotherapy, is lower than in healthy individuals. This lower BMD is less marked in OS with the Ff genotype compared to homozygous FF or ff OS $p=0.049$ ). The genotype that has been correlated in the literature with higher BMD (bbaaTT) is also more frequent among our group of patients with osteosarcoma. Conclusions: Children and adolescents with bone tumours in remission have a lower BMD than healthy individuals. The group of patients with OS have an increased frequency of the Ff genotype, which is statistically related to a higher BMD at remission compared to homozygotes (FF or ff). Patients with OS who have the genotype bbaaTT seem to have a higher BMD at remission.

P1-411 Bone, $\mathrm{Ca}$, PTH and Vitamin D

Bone Mineral Density and Body Composition in Children with Precocious Puberty (CPP) before, during, and after Stop of Treatment with GnRH-Agonist I.M. Van Der Sluis*1; A.M. Boot*1; E.P. Krenning ${ }^{* 2}$; S.L.S. Drop ${ }^{1}$; S.M.P.F. De Muinck Keizer-Schrama ${ }^{1}$

${ }^{1}$ Sophia Children's Hospital, Rotterdam; ${ }^{2}$ University Hospital

Dijkzigt, Rotterdam, The Netherlands

Introduction: During puberty bone mineral density (BMD), body composition (BC) and height change markedly. Treatment (Rx) of CPP with GnRHagonist causes a decline in gonadal sex steroids. Aim: To evaluate BMD and BC in children with CPP during and after GnRH-agonist therapy. Methods: 49 Patients ( 5 boys and 44 girls) with CPP participated, all treated with depot leuprolide-acetate. The mean age at start: 8.3 years (range $2.8-11.9$ ). BMD of lumbar spine (LS) and total body (TB), body composition (DEXA, Lunar), and biochemical bone parameters were assessed. Bone Mineral Apparent Density $\left(\mathrm{BMAD}=\mathrm{BMD}_{\mathrm{LS}}\left[4 /\left(\pi^{*}\right.\right.\right.$ width $\left.\left.)\right]\right)$ was calculated to correct for bone size. Results are expressed as SD Scores (SDS). Results: Table presents SDS during $\mathrm{Rx}$ (months). Mean (SD).

\begin{tabular}{llllll}
\hline & $\begin{array}{l}\mathrm{t}=0 \\
(\mathrm{n}=46)\end{array}$ & $\begin{array}{l}\mathrm{t}=6 \\
(\mathrm{n}=47)\end{array}$ & $\begin{array}{l}\mathrm{t}=12 \\
(\mathrm{n}=45)\end{array}$ & $\begin{array}{l}\mathrm{t}=24 \\
(\mathrm{n}=39)\end{array}$ & $\begin{array}{l}\mathrm{t}=36 \\
(\mathrm{n}=19)\end{array}$ \\
\hline BMD $_{\mathrm{LS}}$ & $0.60(1.19) \#$ & $0.70(1.29) \#$ & $0.65(1.12) \#$ & $0.48(1.14) \#$ & $0.25(1.15)$ \\
BMAD $_{\mathrm{LS}}$ & $0.29(1.18)$ & $0.38(1.31)$ & $0.41(1.17) \#$ & $0.38(1.10) \#$ & $-0.05(1.14)$ \\
BMD $_{\mathrm{TB}}$ & $0.06(1.45)$ & $0.33(1.28)^{*}$ & $0.50(1.13) \# *$ & $0.38(1.17)$ & $-0.11(1.27)^{*}$ \\
LBM & $0.81(1.52) \#$ & $0.68(1.50) \#^{*}$ & $0.71(1.33) \# *$ & $0.49(1.57)^{*}$ & $0.37(1.55)^{*}$ \\
\% fat & $0.44(1.19) \#$ & $0.96(1.25) \# *$ & $1.28(1.21) \# *$ & $1.31(1.32) \# *$ & $1.13(1.01)^{*}$ \\
Height & $1.01(1.36) \#$ & $0.92(1.50) \#$ & $0.81(1.51) \#$ & $0.95(1.45) \# *$ & $0.57(1.78)^{*}$ \\
\hline
\end{tabular}

\# Sign. diff. from zero, ${ }^{*}$ sign. diff. compared to $t=0 . \mathrm{LBM}=$ Lean body mass.

During Rx: BMD corrected for bone age was sign. lower than normal, $\mathrm{BMD}_{\mathrm{TB}}$ increased. Parameters of bone turnover (alkaline phosphatase, anorganic phosphate, PICP, ICTP, osteocalcin and 1,25 diOH vitamin D) decreased sign. After stop of Rx ( 1 yr: $n=17$ and 2 yrs: $n=9)$ BMD, BMAD ${ }_{\text {LS }}$ and LBM did not change, but \%fat decreased. Conclusion: Although children with CPP have normal BMD for chronological age, their BMD for bone age is decreased. During treatment, BMAD $\mathrm{LS}_{\mathrm{LS}}$, BMD and bone turnover decreased sign. LBM decreased and \%fat increased during treatment. Long-term follow-up is required for further evaluation of BMD and body composition after cessation of therapy, to answer the question whether these children will reach their optimal peak bone mass.

\section{P1-412 Bone, Ca, PTH and Vitamin D \\ Real Time PCR for Assessment of 1,25 Dihydroxy Vitamin D Receptor (VDR) Function in Cultured Skin Fibroblasts \\ E.M. Nitsche ${ }^{* 1} ;$ H. Wagner ${ }^{* 1} ;$ S. Nissen ${ }^{* 1} ;$ N. Getschmann ${ }^{* 1}$; O. Hiort ${ }^{1} ; K_{\text {K. }}$ Kruse $^{1}$ \\ ${ }^{1}$ Klinik für Kinder- und Jugendmedizin, Lübeck, Germany}

Background: Dose dependent induction of 25 OH-Vitamin D-24 Hydroxylase (24-OHase) activity by $1.25(\mathrm{OH}) 2$-vitamin D3 (1.25 D3) in cultured skin fibroblasts is established for investigation of VDR function in vitro. Northern Blot analysis recently showed 24-OHase to be upregulated by 1.25 D3 on mRNA level in skin fibroblasts of healthy individuals. We applied a quantitative PCR-based method, real time PCR, for faster and easier functional assessment of intact and defective VDR in vitro. Patients and Method: Skin fibroblasts were obtained from a patient with vitamin D resistant rickets and a healthy control. The fibroblasts were cultured with increasing doses $(0-10 \mathrm{nM})$ of 1.25 D3 as described before and total RNA harvested after six hours (1.2). The RNA of each condition was analyzed by RT-PCR using specific primers for qualitative expression of VDR and 24-OHase mRNA. Real time PCR using specific primers and internal probe and Northern Blot using a 319 Bp radioactive DNA probe were used for semiquantitative analysis of 24-OHase expression. Results: By RT PCR of the coding region of the VDR mRNA, fragments of the right length could be amplified from RNA samples of both fibroblast populations. After 1.25 D3 treatment, RT PCR for 24-OHase was positive in all samples. Real time PCR following reverse transcription showed dose dependent stimulation of 24-OHase expression in both populations. Maximum stimulation in the fibroblasts of healthy controls was significantly higher than in fibroblasts of the patient with vitamin D resistance. This result was confirmed by Northern Blot using $10 \mathrm{~g}$ total RNA of each condition. Conclusion: The results of our study confirm the observation, that 24-OHase stimulation by $1.25 \mathrm{D} 3$ is regulated on mRNA level. The results of real time PCR and Northern Blot equally showed the significant difference in 1.25 D3 stimulated 24OHase levels that has been published for enzyme activity measurement. We therefore conclude, that real time PCR is an easy to perform, valid and rapid method for assessing induction of 24-OHase in vitro as an expression of VDR function. 
P1-413 Bone, Ca, PTH and Vitamin D

\section{Autosomal Dominant Hypocalcemia: Phenotype: Genotype Relationships}

A. Lienhardt*1; M.-L. Kottler*2; M. Garabédian*3; Introduced by M.O. Savage

${ }^{1}$ Limoges, France; ${ }^{2} \mathrm{CHU}$ Pitié-Salpétrière, Paris, France; ${ }^{3}$ Hôpital St Vincent de Paul, Paris, France

Autosomal dominant hypocalcemia $(\mathrm{ADH})$ and sporadic hypoparathyroidism have been related to activating mutations of the Calcium Sensing Receptor gene (CaSR), a GPCR involved in calcium metabolism. Objective: To evaluate phenotype: genotype relationships in $\mathrm{ADH}$ associated with CaSR mutations. Patients and Methods: A large French collaborative study included 4 unrelated kindred presenting ADH and 3 children with sporadic hypoparathyroidism (18 affected and 39 unaffected members). Clinical and biological features were studied. In affected probants, the CaSR gene was studied using direct sequence analysis of PCR products. Segregation analysis of a mutation was conducted using either enzyme restriction sites followed by gel electrophoresis or direct sequencing of the DNA. Results: Seven CaSR mutations have been identified, 3 involving the CaSR extracellular domain (ECD) - L125P, C129F, P221L - and 4 the intracellular domain (ICD) - F788C, E799K, A843K, DelS895. An homozygous mutation was found in one patient and heterozygous mutations were found in all the others patients. A total concordance between genotype (presence or absence of mutation) and biological phenotype (presence or absence of hypocalcemia) was observed. The severity of the initial clinical features was negatively correlated to the initial serum calcium levels $(r=-0.73, p=0.007)$ but not to the iPTH levels. However, at the time of diagnosis, affected patients had various clinical presentations ranging from non symptomatic forms to mild signs of hypocalcemia (muscles cramps, asthenia) or severe neonatal seizures. Moreover, affected members harbouring a same mutation could exhibit different clinical presentations and calcium levels. In addition, the 3 patients with sporadic hypoparathyroidism had severe neonatal seizures and mutations are located either in the ECD, the ligand binding domain or in the ICD, involved in the signal transduction, indicating no correlation between the phenotype and the functional part of the CaSR which is altered. Conclusions: ADH is associated with a wide range of the clinical presentation of hypocalcemia. No CaSR-mutation: phenotype relationships for ADH or sporadic hypoparathyroidism could be demonstrated in our study.

P1-414 Bone, Ca, PTH and Vitamin D

\section{Bone Mineral Density in Transsexuals Treated with Cross-Sex Hormones}

S.U. Kalintchenko*1; G.I. Kozlov*1;O.V. Remisov*1; Introduced by A. Tiulpacov

${ }^{1}$ Endocrinological Research Center, Moscow, Russian Federation

Introduction: Sex steroids play important role in regulation of bone metabolism and bone mineral density (BMD). Hypogonadism is a substantial risk factor for bone loss. What do reflect adequacy of hormone substitution for maintenance of bone mass in hypoganodal patients? Patients and Methods: We monitored BMD for 100 transsexuals underwent gonadectomy, with a mean age (sd) of $22.4 \mathrm{yr}$ (range, 17-31): 30 with female-to-male (F-M) TS, treated with oestrogens and 30 with male-to-female (M-F) TS, treated with androgens. The duration of cross-sex hormone administration after gonadectomy was $3.5 \pm 1.1 \mathrm{yr}$. Results: In oestrogens-treated M-F BMD don't change. But all M-F receved high doses of oestrogens ( 15 patients had the increase of the prolactin level). The serum level of estradiol was normal, the serum level of $\mathrm{LH}$ was normal or decreased. In androgentreated F-M BMD had decreased significantly. The serum level of total testosterone in F-M was normal, but serum level of LH was elevated. The change of BMD correlated inversely with serum level of LH in F-M. Conclusion: In cases of hypogonadism serum level of $\mathrm{LH}$ is a better marker of adequate substitution and prevention of bone loss than serum levels of sex steroids.

P1-415 Bone, Ca, PTH and Vitamin D

\section{Albright's Hereditary Osteodystrophy: Seven Novel} Mutations in the GNAS1 Gene

W. Ahrens ${ }^{* 1} ;$ O. Hiort ${ }^{2} ;$ T. Kirschner*2; K. Kruse ${ }^{2}$

${ }^{1}$ Medical University of Lübeck, ${ }^{2}$ Lübeck, Germany

Albright's hereditary osteodystrophy (AHO) is a genetic disorder characterized by phenotypic signs of brachydactyly, subcutaneous calcification, short stature, obesity, and mental retardation. Patients with AHO, pseudohypoparathyroidism, and associated endocrinopathies like hypothyroidism (PHP Ia) can be differentiated from patients showing signs of $\mathrm{AHO}$ without hormone resistance (pseudoPHP). Both autosomal dominant inherited disorders can occur in the same family, a genomic imprinting is described. Patients with PHP Ia and pseudo-PHP show a reduced activity of the adenylyl cyclase stimulating protein Gsalpha (Gs $\alpha$ ). Different inactivating mutations have been identified in the gene encoding Gs $\alpha$ (GNAS1) without real 'hot spots'. Patients and Methods: Six children and one adult with $\mathrm{AHO}$ and hypothyroidism from different families were investigated. Five patients (P 1, 2, 4, 6, 7) were classified as PHP Ia, calcium metabolism of two boys (P 3, 5) showed no alteration until now. Compared to healthy controls, Gs $\alpha$ protein activity in erythrocyte membranes was analyzed in vitro adapted from the method of Levine et al. (Biochem Biophys Res Commun 1980, 94: 1319-1324). Using genomic DNA, exons (E) 1-13 of GNAS1 were investigated by PCR-SSCA and direct sequencing of PCR-products. Results: Reduced Gs $\alpha$ protein activity and different novel mutations in the GNAS1 gene were found in the seven patients: P1: Gsa activity 67\%, Ala102Val (E4); P2: 54\%, Ala102Gln (E4); P3: 58\%, Arg231Cys (E9); P4: 48\%, Ala298Pro (E11); P5: 55\%, Pro313Leu (E11); P6: 64\%, splice site mutation I6/E7; P7: 56\%, frame shift mutation with stop codon in position 309 (E11). In addition, the mothers of P 1, 2, 5, and 6 with pseudo-PHP and the sister of P6 with PHP Ia were investigated. They showed reduced Gs $\alpha$ activity and the same mutation in the GNAS1 gene compared to their children whereas in healthy relatives with normal Gs $\alpha$ activity no alterations were detected. Conclusions: Five novel missense and two nonsense mutations in the GNAS1 gene of patients with AHO were detected. The results confirm that there seems to be no 'hot spot' for these inactivating mutations in the GNAS1 gene. A clear cut genotype/phenotype correlation is not possible.

P1-416 Bone, Ca, PTH and Vitamin D

Pubertal Gymnasts Increase Whole Body Mineral Content in Contrast to Swimmers during One Year of Intensive Training

R. Damsgaard*1; C. Mølgaard*2; J. Bencke*3; J Müller ${ }^{1}$

${ }^{1}$ University Hospital of Copenhagen; ${ }^{2}$ Royal V' Agricultural University; ${ }^{3}$ University of Copenhagen, Copenhagen,

Denmark

It is well established that bone mineral content (BMC) is increased in physical active children compared to non-active children. However, a few studies of girls in gymnastics and ballet have shown that intensive training may have an adverse effect on BMC. Objective: To evaluate changes in whole body BMC in pubertal athletes during one year of training at a competitive level. Subjects and Methods: 20 female swimmers (Sw) (median age 12.1, range 10.6-13.4) and 19 female gymnasts (Gy) (median age 12.2, range 10.8-13.2) took part in the study. Whole body BMC and bone area (BA) were determined twice with one year apart by DXA (Hologic 1000/W). The Mann-Whitney test and Wilcoxon signed-rank test were used to compare the results of the two measurements. Results: Median z-scores for height, BA according to height, $\mathrm{BMC}$ according to $\mathrm{BA}$, and $\mathrm{BMC}$ were calculated using Danish reference data (Table). Median training hours per week in both sports was $10 \mathrm{~h}$ (range 5-18). Swimmers were taller than gymnasts at both investigations $\left({ }^{1} \mathrm{p}<0.01\right.$ and ${ }^{2} \mathrm{p}<0.04$, respectively). Conversely, gymnasts had greater bone mass corrected for size (BMC-for-BA) than swimmers $\left({ }^{3} p<0.01\right)$. No significantly changes in median $\mathrm{z}$-scores were found in any of the bone-related variables during the investigation period. The pubertal development, estimated by breast stage, was not different between the groups.

\begin{tabular}{|c|c|c|c|c|c|}
\hline & Investigation & Height & BA-for-height & BMC-for-BA & $\mathrm{BMC}$ \\
\hline \multirow[t]{2}{*}{ Sw } & 1 & $0.36^{1}$ & -0.82 & -0.32 & 0.15 \\
\hline & 2 & $0.22^{2}$ & -0.44 & $-0.56^{3}$ & 0.18 \\
\hline \multirow[t]{2}{*}{ Gy } & 1 & $-0.65^{1}$ & 0.06 & 0.12 & -0.82 \\
\hline & 2 & $-0.46^{2}$ & 0.11 & $0.37^{3}$ & -0.60 \\
\hline
\end{tabular}

Conclusion: We have shown that girl gymnasts, training at a competitive level, have normal whole body BMC when corrected for their short bones. We also showed that one year of training did not affect BMC adversely in gymnasts. In fact, the high impact loading in gymnastics seems to have a positive effect on bone mass (BMC-for-BA) when compared to the non-impact loading in swimming. 
P1-418 Bone, Ca, PTH and Vitamin D

Reduced Bone Strength as a Consequence of Undermineralisation in GH Deficient Transgenic (TGR) Rats

J. Warner*1; T. Wells*2; C. Elford ${ }^{1} ;$ B. Evans ${ }^{1} ;$ S. Evans ${ }^{* 1}$ J. Gregory 1

${ }^{1}$ University of Wales College of Medicine; ${ }^{2}$ School of Bioscience,

Cardiff, United Kingdom

Introduction: Growth hormone deficiency (GHD) in childhood is associated with osteopenia, though little is known about its effects on bone strength and fracture risk. The Tgr rat is a new model for GHD in which expression of hGH in growth hormone release factor neurones produces a 30\% reduction in $\mathrm{GH}$ secretion and skeletal growth similar to $\mathrm{GH}$ insufficiency in childhood. The aim of this study was to measure the effect of GH insufficiency in Tgr rats on bone strength and bone mineral content (BMC) by comparison with normal (AS) control rats and examine associations with anthropometric measures. Methods: The left femur was dissected from male and female Tgr and AS rats (aged 15-23 weeks). Bone strength was measured by three point bending to the point of fracture (failure load). BMC was measured by dual energy X-ray absorptiometry (DEXA). BMC was adjusted for bone area (BA), femur length, body weight, age and sex using multiple regression and data from the AS animals and expressed as standard deviation scores (SDS). Results: Measurements were made in 15 male and 14 female Tgr rats and 20 male and 13 female $\mathrm{AS}$ rats. BMC was correlated with $\mathrm{BA}(\mathrm{R} 2=0.98, \mathrm{p}<0.001)$ with significant differences in the slope between AS and Tgr animals (coefficient $=-0.063$ ( $\mathrm{SE}=$ $0.016), \mathrm{p}<0.001)$. Tgr rats were severely osteoporotic with a mean (SD) BMC SDS of $-4.1(1.2)$ and -3.5 (1.1) for males and females respectively. Tgr rats had a reduced mean (SD) failure load compared to AS controls (males: 91.0 $(10.5)$ vs. $140.4(19.9) \mathrm{N}$, females $91.0(7.8)$ vs. $117.8(7.8) \mathrm{N}$ respectively, $\mathrm{p}<$ $0.001)$. Failure load was correlated with BMC SDS, $r=0.63$ for all animals combined, $\mathrm{p}<0.001$. Conclusion: Bone strength and BMC are significantly reduced in $\mathrm{GH}$ insufficient Tgr rats. The differences in the relationship between BMC and BA suggest that the Tgr bones are undermineralised compared to AS animals. Greater bone strength was associated with increasing BMC SDS. These data suggest that GHD is associated with undermineralisation and subsequent reduced bone strength. The clinical implication is that fracture threshold is reached at a lower level in GHD, leading to increased fracture risk. It remains to be seen if $\mathrm{GH}$ therapy can restore bone mineralisation and strength to normal in Tgr animals.

\section{P1-419 Bone, Ca, PTH and Vitamin D \\ Bone Mineral Density and Metabolism in HIV-Infected Children}

F. Antoniazzi ${ }^{1}$; A. Dall'Agnola*1; E. Concia*1; R. Luzzati*1. S. Lauriola*1 ; G. Zamboni*1;F. Mengarda*1;F. Bertoldo*1; ${ }^{*}$. Tatò ${ }^{1}$

1 University of Verona, Verona, Italy

As human immunodeficiency virus (HIV) has effects on nutritional status, producing malnutrition, wasting and depletion of lean body mass, the present pilot study was conducted to obtain preliminary information on the effect of HIV on bone metabolism of HIV-infected children. In thirteen prebubertal HIV-positive patients (4 males; 16 months-13 years of age) nutritional assessement, auxological parameters (weight, height, weight and height velocity, Tanner staging), insulin-like growth factor (IGF-I) production, GH secretion (after GH-RH stimulation), bone age and bone densitometry (by DEXA), were determined before the start of antiretroviral therapy with protease inhibitors. Twelve children (4 males), machted for age and sex, were studied as controls. IGF-I was determined by RIA. Bone age was measured radiologically on the left hand and wrist. Bone densitometry measurement was performed by total body and spinal BMD (L2-L4) by DEXA (Lunar). Nutrional status, auxological parameters, hypophyseal function and GH secretion were adequate for age and sex. In all HIV-infected children IGF-I levels showed a significant positive correlation with DEXA values $(\mathrm{r}=0.687 ; \mathrm{p}<0.0005)$. In 6 patients blood IGF-I levels $(99.17 \pm 58.76 \mu \mathrm{g} / \mathrm{l})$ and DEXA $\left(0.59 \pm 0.15 \mathrm{~g} / \mathrm{cm}^{2}\right)$ values were lower than in controls $(p<0.0261)$. Two patients of this group suffered from severe bone pains, and 1 had spontaneous fracture of the left clavicule. In the remaining 7 HIV-infected patients IGF-I levels $(279.71 \pm 162.45 \mu \mathrm{g} / \mathrm{l})$ and DEXA values $\left(0.940 .13 \mathrm{~g} / \mathrm{cm}^{2}\right)$ were in the range of controls. We hypothesize that HIV itself could have negative side-effect on mineral metabolism, by riducing IGF-I pro- duction with consequent alteration of bone mineralization. Additional studies are in progress to assess the potential additional risks connected with antiretroviral therapy, as side-effects of protease inhibitors on endocrine-metabolism system have been demonstrated.

\section{P1-420 Bone, Ca, PTH and Vitamin D \\ A Short-Term Double-Blind Study of Serum Osteocalcin in Schoolchildren \\ A.J.Schou ${ }^{* 1} ;$ C. Heuck*2;O.D. Wolthers ${ }^{2}$ \\ ${ }^{1}$ Arhus, Denmark; ${ }^{2}$ Randers, Denmark}

Assessment of serum osteocalcin, which reflects osteoblast activity, may be a sensitive marker of bone formation. In adults bone turnover modulating therapies such as glucocorticoids and vitamin-D have been found to affect circulating osteocalcin levels. Objective: To assess whether short term administration of vitamin-D to healthy children affects serum osteocalcin. Furthermore, the procollagen peptides of bone (type I) collagen turnover: the amino (PINP) and carboxy (PICP) terminal propeptides (formation markers)and the carboxy terminal pyridinoline cross-linked telopeptide (ITCP) (a degradation marker) in serum were measured. Patients and Methods: The study was a doubleblind, placebo-controlled cross-over trial with 4 weeks treatment and 2 weeks run in and washout periods. Twelve girls and 8 boys, all healthy, aged 6.2-13.7 (mean 9.8) years were enrolled. Seventeen were Tanner stage 1, 1 was stage 2, 3 and 4, respectively. Active treatment was $600 \mathrm{IU}(15 \mu \mathrm{g})$ vitamin-D given as one tablet ABCDin ${ }^{\circledR}$ per day. Blood samples were taken on the last day of the treatment periods. Results: Osteocalcin, PINP, PICP and ITCP (mean \pm SEM) were 54(6), 578(56), 437(44) and 13(0.8) $\mu \mathrm{g} / \mathrm{l}$ during vitamin-D treatment; and 52(4), 614(64), 429(41) and 14(0.8) $\mu \mathrm{g} / 1$ during placebo treatment (NS). Conclusion: Short term vitamin-D administration to healthy children does not affect serum osteocalcin or procollagen peptides of bone collagen.

\section{P3-421 Bone, Ca, PTH and Vitamin D \\ Bone Mineral Density in Adult Ullrich-Turner Patients: Preliminary Data of Peripheral Quantitative Computer Tomography \\ S.M. Bechtold ${ }^{* 1} ;$ V. Noelle*1;C. Neu ${ }^{* 2} ;$ S. Dohnhauser*1; E. Schönau ${ }^{2} ;$ H.P. Schwarz \\ 1 University Childrens Hospital, Munich, Germany; ${ }^{2}$ University \\ Childrens Hospital, Cologne, Germany}

Introduction: Adult women with Ullrich-Turner syndrome (UTS) often show radiographic signs of osteopenia. Earlier studies have suggested that osteopenia is a frequent finding in adult UTS patients. In recent studies, however, normal $\mathrm{BMD}$ after growth hormone $(\mathrm{GH})$ therapy was reported. The aim of our study was to investigate BMD in UTS patients after completion of GH therapy. Methods: BMD was measured in 12 adolescent or adult women with UTS at a mean age of 19.2 years (range 17 to 25.4 years). Ten had a karyotype of 45 ,XO and two a mosaic of $45, \mathrm{XO} / 46, \mathrm{XX}$. BMD was selectively measured in trabecular, cortical and total bone using peripheral quantitative computer tomography (pQCT). All patients had been treated with GH for a mean period of 4 years (range 1.4 to 5.7 years). Treatment was started at a mean age of 12.7 years and stopped at the mean age of 16.7 years. Estrogens were substituted at a mean age of 14.1 years and a corresponding mean bone age of 12.4 years. Results: Total bone mineral density measured at the distal radial bone of the nondominant arm was -1.9 SDS, trabecular bone density was -0.2 SDS. Cortical area, cortical thickness and cortical density, however, measured at the proximal forearm, was reduced to $-1.6 \mathrm{SDS},-1.4 \mathrm{SDS}$ and $-2.8 \mathrm{SDS}$, respectively. Even after correction for bone size cortical thickness remained low at -0.96 SDS. Muscular area was not reduced for age and height, but cortical area for muscular area was low at -0.76 SDS. Conclusions: PQCT allows selective measurement of metabolic active cortical and trabecular bone. Cortical density and thickness seem to be more affected in UTS than trabecular bone. Underestimation of cortical density could be partially due to the small size of the patients (partial volume effect). The reduction of cortical thickness is not caused by a reduced muscular area representing muscular mass. We conclude from our preliminary data that cortical area and total BMD in UTS patients treated with growth hormone and estrogens is reduced, whereas trabecular BMD is normal. 
P3-422 Bone, Ca, PTH and Vitamin D

Bone Mineral Density in Children and Adolescents Survivors of Malignant Solid Tumours

C. Azcona ${ }^{1}$; S. Raggio*1; P. Fiz*1; J. Gimeno*1; J. Boan*1; L. Sierrasesumaga*1

${ }^{1}$ Pamplona, Spain

Introduction: The improvement of diagnostic and therapeutic modalities in children with cancer have led to increased survival rates. Although cured of their malignancies, some of these children are at risk of developing late complications after receiving some therapies which can induce hormonal, metabolic and nutritional effects and therefore interfer with normal bone accretion. DualEnergy-X-Ray absorptiometry (DEXA) has been the most common method to assess bone mineral density (BMD) in most of the studies. Up-to-date there are no data of bone mineralization determined by quantitative digital ultrasounds (QUS) in children with cancer. Objectives: (1) To assess BMD in children and adolescents with malignant solid tumours at remission. (2) To compare two methods to assess BMD (DEXA and QUS) in this group of patients. Material and Methods: 53 patients ( 24 females and 29 males) survivors of solid malignant tumours (22 osteosarcomas, 13 Ewings sarcomas, 6 lymphomas, 11 other tumours) were studied. Results are expressed as mean (confidence interval). BMD was assessed by DEXA (HOLOGIC QDR 4500 Elite, Hologic, Inc, Wolthman, Mass.) at lumbar spine and by QUS at the distal metaphysis of proximal phalanxes of the last four fingers of the non-dominant hand, obtaining the mean bone transmission speed of sound (SOS). A DMB Sonic 12000 ultrasound densitometer was used. Results: The mean age of patients at BMD determination was 16.5 years $(15.1 ; 17 ; 9)$. Body mass index at BMD determination was $21.6(20.2 ; 23.0)$. Mean time interval since completion of therapy was 4.75 years. Lumbar spine BMD (SDS) determined by DEXA $(n=44)$ was $-0.71(-0.42 ;-1.01)$. SOS at the phalanxes assessed by QUS $(n=35)$ was $1966.37 \mathrm{~m} / \mathrm{s}(1929.9 ; 2002.9)$. BMD values assessed by DEXA $\left(\mathrm{g} / \mathrm{cm}^{2}\right)$ and QUS $(\mathrm{m} / \mathrm{s})$ were significantly correlated $(\mathrm{n}=27 ; \mathrm{r}=0.71 ; \mathrm{p}<0.0001)$. Conclusions: Patients survivors of malignant solid tumours have a lower BMD than controls, assessed by two different methods which show a significant correlation. QUS is an easy, fast and inexpensive method to measure BMD in children and adolescents, without exposing the patient to sources of irradiation.

P3-423 Bone, Ca, PTH and Vitamin D

The Assessment of Bone Mineral Density in Children with Growth Hormone Deficiency

D. Daniec-Janus ${ }^{* 1} ;$ H. Dziatkowiak*1;E. Czerwinski*1; Introduced by B. Rymkiewicz-Kluczynska

${ }^{1}$ Polish-American Children Hospital, Krakow, Poland

Children with isolated growth hormone deficiency or with multiple pituitary hormone deficiency have reduced bone mineral density. Administration of recombinant human growth hormone ( $\mathrm{rhGH}$ ) is known to stimulate bone turnover. Objective: The assessment of bone mineral density in children with growth hormone deficiency ( GHD) before treatment and treated with rhGH. Patients and Methods: We assessed 62 children: 21 girls: 17 rhGH treated $1-10$ years ( 8 before the onset of puberty aged 6-18 years and 9 after the onset of puberty aged 11-20 years). There were 4 girls with GHD before rhGH treatment aged 5-12 years. We assessed 41 boys: 29 rhGH treated $1-16$ years (13 before the onset of puberty aged 7-20 years and 16 boys after the onset of puberty aged $12-23$ years. There were 12 boys aged $5-20$ years with diagnosed GHD before treatment. The control assessment of bone mineral density after 1 year of observation was made in a group of 30 children ( 11 girls and 19 boys). The evaluation of the BMD of lumbar spine was made with dual-energy X-ray absorptiometry. Lumbar spine BMD was calculated to correct for bone age. Pubertal stage was assessed according to Tanner score. Results: In pretreatment children reduced lumbar BMD was found in 2 out of 4 girls and 8 out of $12(66 \%)$ boys. In the group of rhGH treated children before the onset of puberty reduced BMD was found in 5 out of $13(38 \%)$ boys and 4 out of $8(50 \%)$ girls. In the group of pubescent children reduced BMD was found in 5 out of 16 $(31 \%)$ boys and 2 out of $9(22 \%)$ girls. In both boys and girls BMD correlated to Tanner stages. After 1 year of observation, treatment with rhGH, Vitamin D3 and Calcium we found an increment of BMD in $73 \%$ of girls and $68 \%$ of boys.
Conclusion: Children with GHD have reduced BMD. In our group of patients growth hormone therapy together with Calcium and Vitamin D3 intake increased bone mass. During puberty the increment of BMD was higher in girls. The increase in BMD correlated positively with growth velocity and body mass index in both boys and girls.

P3-424 Bone, Ca, PTH and Vitamin D

Effect of Long-Term Gluten-Free Diet on Bone Density and Bone Metabolism in Children with Celiac Disease: A Longitudinal Study

S. Mora*1; S. Beccio*2; G. Barera*2; L. Menni*2;M.C. Proverbio*2; G. Weber ${ }^{2}$; G. Chiumello 2

${ }^{1}$ Laboratory of Pediatric Endocrinology, Milan, Italy; ${ }^{2}$ Milan, Italy

Osteoporosis is a frequent complication of celiac disease. We evaluated the impact of long-term gluten-free diet (GFD) initiated during childhood on bone density and metabolism. Nineteen celiac patients were studied at diagnosis (mean age $9.3 \pm 0.7 \mathrm{yrs}$ ), and after $4.3 \pm 0.6 \mathrm{yrs}$ of GFD. We also studied 211 healthy children, aged 1.58 to $19.2 \mathrm{yrs}$, as control group. Bone mineral density BMD) was measured in celiac patients at diagnosis and after GFD, while bone metabolism indexes were measured only after dietary treatment. Bone density values were obtained with a dual-energy x-ray absorptiometer (DPX-L, Lunar Radiation Corp.) at the lumbar spine and in the whole skeleton. Intact parathyroid hormone (PTH) was measured in serum by an immunoradiometric assay. Bone formation was assessed by serum measurements of bone-specific alkaline phosphatase (BALP), and bone resorption was estimated by urine measurements of N-terminal telopeptide of type I collagen (NTx). Multivariate analyses were performed to evaluate the differences between celiac patients and control subjects, after controlling for confounding variables (age, gender, anthropometric measurements). At diagnosis mean spinal BMD of celiac patients was 0.708 $\pm 0.034 \mathrm{~g} / \mathrm{cm}^{2}$, and mean whole body BMD was $0.875 \pm 0.02 \mathrm{~g} / \mathrm{cm}^{2}$. Bone density values of celiac patients were significantly lower than those of control subjects $(\mathrm{p}=0.015$, and $\mathrm{p}=0.04$, respectively). After GFD the mean spinal BMD was $0.9040 .219 \mathrm{~g} / \mathrm{cm}^{2}$, and whole body BMD was $1.007 \pm 0.123 \mathrm{~g} / \mathrm{cm}^{2}$. The differences of BMD between patients and control subjects were no more significant ( $\mathrm{p}=0.64$, and $\mathrm{p}=0.55$, respectively). None of the patients on GFD showed elevated values of PTH $(15.7 \pm 4.2 \mathrm{pg} / \mathrm{ml})$. Celiac patients on GFD showed BALP $(110.2 \pm 67.2 \mathrm{U} / \mathrm{l})$ and NTx levels $(261.9 \pm 187.8 \mathrm{nmol} \mathrm{BCE} /$ $\mathrm{mmol}$ creatinine) significantly higher that those of control subjects $(\mathrm{p}=0.009$, and $p=0.01$, respectively). In conclusion, the present study confirms a marked reduction of bone density in untreated celiac patients, which is completely restored after long-term GFD. Our data seem to indicate that the normal bone density is maintained at the expenses of a greater bone turnover (i.e. increased bone formation and resorption), not sustained by secondary hyperparathyroidism. 
P3-425 Bone, Ca, PTH and Vitamin D

The Risk Factors for Vitamin D Deficiency in Breastfed Newborns and Their Mothers

N. Andiran ${ }^{* 1} ;$ N. Yordam $1 ;$ A. Özön*1

${ }^{1}$ Hacettepe University, Ankara, Turkey

Objective: To evaluate the current vitamin D status of healthy breastfed newborns and their nursing mothers. Patients and Methods: The study was conducted during the period of October-November 1999. 54 healthy breastfed newborns randomly chosen among those recalled from the "congenital hypothyroidism screening program' in our hospital and their nursing mothers were evaluated. Blood were drawn from the babies and their mothers for 25-hydroxy vitamin-D (25-OHvitD), calcium, phosphorus and alkaline phosphatase measurements. Relationship between 25-OHVitD levels, and socioeconomic status, educational level and dressing habits (covered vs uncovered) were also analysed. Covered as a dressing habit meant covering the hair and sometimes part of the face with a scarf (different than veiled) and wearing clothes that cover the arms and legs completely. In mothers with a serum 25-OHvitD level below $25 \mathrm{nmol} / \mathrm{l}$ bone mineral density was measured with dual energy x-ray absorptiometry to determine the extent of bone mineralisation. Results: Serum calcium, phosphorus, and alkaline phosphatase levels were within the normal levels for all newborns and their mothers. Mean serum 25-OHVitD level of the mothers was $29,1110.47 \mathrm{nmol} / 1.85 \%$ of the mothers

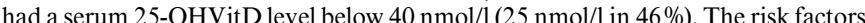
for low maternal serum 25-OHvitD level were: Low socioeconomic class $(\mathrm{OR}=$ $8.1, \mathrm{p}=0.000)$, covered $(\mathrm{OR}=4.3, \mathrm{p}=0.023)$ and low educational level $(\mathrm{OR}=3.5$, $\mathrm{p}=0.033$ ). Mean serum 25-OHVitD level of the newborns was $18,628.00 \mathrm{nmol} / 1$. Eighty percent of the newborns had serum 25-OHvitD levels lower than $25 \mathrm{nmol} / 1$. There was significant correlation between the serum 25-OHvitD levels of the newborns and their mothers $(\mathrm{r}=0.63, \mathrm{p}=0.01)$. The most important risk factor for low serum 25-OHvitD level in the newborn was the maternal level of 25-OHvitD lower than $25 \mathrm{nmol} / 1(\mathrm{OR}=15.2, \mathrm{p}=0.002)$, while the calculated risk of a low 25 OHVitD level was $6.8(\mathrm{OR}, \mathrm{p}=0.011)$ in babies of covered mothers. Bone mineral density revealed osteopenia in $40 \%$ of the women with a low VitD level. All women with osteopenia were from low socioeconomic class and $80 \%$ of them was covered. Conclusion: Vitamin D deficiency is still a common problem in reproductive women and their babies in developing countries at the gateway to a new millennium.

P3-426 Bone, Ca, PTH and Vitamin D

\section{Cortical Bone Modeling and Remodeling in Idiopathic} Juvenile Osteoporosis (IJO)

F. Rauch ${ }^{1} ;$ R. Travers ${ }^{* 2}$; F.H. Glorieux ${ }^{* 2}$

${ }_{1}^{1}$ University Childrens Hospital, Koeln, Germany; ${ }^{2}$ Shriners Hospital for Children, Montreal, Canada

We have previously shown that IJO is characterized by a decreased cancellous bone volume and a very low bone formation rate on cancellous surfaces. Whether IJO similarly affects cortical bone is unknown. We therefore compared tetracycline double labeled transfixing iliac crest bone biopsies from 8 children with typical clinical features of IJO (6 girls; age 10 to 12 years) and from 9 children ( 4 girls; age 9 to 12 years) without metabolic bone disease. Histomorphometric parameters were measured semi-automatically using a digitizing tablet and the Osteomeasure ${ }^{\circledR}$ software (Osteometrics Inc., Atlanta, USA). No differences in intracortical remodeling activity were detected. Both structural parameters reflecting intracortical remodeling (cortical porosity, active canal diameter, quiescent canal diameter) and bone surface based remodeling parameters (osteoid, osteoblast, mineralizing, osteoclast and eroded surfaces, bone formation rate) were similar in IJO patients and controls ( $p>0.2$ each, t-test). While the internal cortex of the biopsy core was thinner in IJO patients than in controls $(660 \pm 170 \mu \mathrm{m}$ vs. $980 \pm 320 \mu \mathrm{m} ; \mathrm{p}=0.02)$, there was no difference in the width of the external cortex $(p=0.36)$. As in growing children both cortices exhibit an external modeling drift, the difference in internal cortical width points to a decreased modeling activity on the endocortical surface of the internal cortex. In fact, bone formation rate on this surface was decreased by $48 \%$ in IJO patients $\left(82 \pm 45 \mu^{3} / \mu m^{2} / y\right.$ vs. $\left.159 \pm 162 \mu \mathrm{m}^{3} / \mu^{2} / \mathrm{y}\right)$. However, this difference did not achieve statistical significance $(\mathrm{p}=0.21)$ due to the high variability of bone formation rate on modeling surfaces. In conclusion, the disturbance of bone remodeling in IJO is limited to cancellous bone, but there may be a modeling defect affecting the internal cortex. Thus, the process causing IJO appears to mainly affect bone surfaces which are in contact with the bone marrow cavity. This may explain, why fractures in IJO predominantly occur in vertebral bodies and long bone metaphyses rather than in diaphyses.
P3-427 Bone, Ca, PTH and Vitamin D

Osteopetrosis and Renal Tubular Acidosis with Normal Carbonic Anhydrase II Activity

N. Kandemir ${ }^{1} ;$ R. Topaloglu*1; M. Yurdakök ${ }^{* 1} ;$ N. Yordam $^{1}$; A. Bakkaloglu 1 ; W.S. Sly ${ }^{* 2}$

${ }^{1}$ Hacettepe University, Ankara, Turkey; ${ }^{2}$ St Louis University, St. Louis, Mo., USA

Carbonic anhydrase II deficiency (CAII) is an autosomal recessive disorder manifest by osteopetrosis, renal tubular acidosis and cerebral calcification. It is usually not recognized until late infancy or early childhood. We present a family who has a boy with osteopetrosis and renal tubular acidosis and a girl with only renal tubular acidosis. The parents were first cousins. The first child of the family was admitted to our hospital with respiratory distress, difficulty in feeding and hypocalcemia when he was 10 days old. Generalized increased bone density, with a 'bone within a bone' appearance in the tubular bones was present and osteopetrosis was diagnosed. He had anemia $(\mathrm{Hb} 7.2 \mathrm{~g} / \mathrm{dl})$ and leucocytosis $\left(32 \times 10^{9}\right)$. Peripheral blood smear showed numerous promyelocytes, myelocytes, metarnyelocytes and normoblasts. His blood $\mathrm{pH}$ was 7.25 , bicarbonate content $11.2 \mathrm{mmol} / 1$, urine $\mathrm{pH}$ 7.5. He had severe hypokalemia as low as $1.5 \mathrm{mEq} / \mathrm{l}$ and urinary $\mathrm{Ca} / \mathrm{Cr}$ was 0.45 (normal $<0.2$ ). Renal USG revealed increased renal medullar echogenicity. Although he was on K supplementation and $\mathrm{HCO}_{3}$ treatment he had severe growth retardation. His weight was $3500 \mathrm{~g}$ and height was $53 \mathrm{~cm}$ when he died at 12 months old. His sister diagnosed renal tubular acidosis when she was 3.5 months old. Her blood pH was 7.28 , bicarbonate content $13.1 \mathrm{mmol} / 1$, urine $\mathrm{pH} 7$, urinary $\mathrm{Ca} / \mathrm{Cr} 0.18$. She had hypopotassemia and medullar nephrocalcinosis was found in renal USG. Potassium supplementation and $\mathrm{HCO}_{3}$ treatment has commenced. Her skeletal $\mathrm{X}$ ray was normal. She had less severe growth failure than her brother. Her weight was $6000 \mathrm{~g}$ and height was $70 \mathrm{~cm}$ when she was 12 months old. Activity of CAII was found normal in both patients and parents. This is the first report of patients with osteopetrosis and renal tubular acidosis with normal CAII activity. Two different genetic diseases in this family is likely but the possibility of a single unknown defect that can cause both pathologies can not be completely ruled out.
P3-428 Bone, Ca, PTH and Vitamin D

\section{Mutations in THA Gs $\alpha$ Gene in 8 Families with} Albright's Hereditary Ostedystrophy

G. Mantovani*1; R. Romoli*1; G. Weber ${ }^{2}$; V. Brunelli*3; E. De Menis* ${ }^{*}$; P. Beck-Peccoz*1;A. Spada*1

${ }^{1}$ Institute of Endocrine Sciences, Milano, Italy; ${ }^{2}$ Ospedale San Raffaele, Milano, Italy; ${ }^{3}$ Pediatria-Ospedale Buzzi, Milano, Italy;

${ }^{4}$ Ospedale Regionale Veneto, Treviso, Italy

Albright hereditary osteodystrophy (AHO) is an inherited disorder characterized by short stature, obesity, rounded face, brachydactyly and subcutaneous calcifications which can be found as an isolated defect (pseudopseudo-hypoparathyroidism: PPHP) or associated with multiple hormone resistance (pseudohypoparathyroidism type Ia: PHP Ia). In many cases (about 50\%) inactivating mutations in the Gs $\alpha$ gene can bedetected in affected subjects. Objective: We collected clinical, biochemical and molecular data in 8 unrelated families affected by PHP Ia and PPHP in order to study the prevalence of Gs $\alpha$ mutations in these patients. Patients and Methods: Eight families, 4 of which presenting members with clinical features of PHP Ia and 4 with isolated PPHP, were screened for mutations in the 13 exons of the Gs $\alpha$ gene, using polymerase chain reaction (PCR) and direct sequencing of the amplified products. Results: We detected mutations in the Gs $\alpha$ gene in the affected members of the 4 families in which PHP Ia was present. In 2 families, 2 previously reported deletions (a 4-bp deletion in exon 7 and a 1-bp deletion in exon 5) known to be responsible for a partial functional defect of the Gsa protein, were found. In the other 2 families, 2 novel frame shift deletions were identified in exons 1 and 11 involving 1 and $2 \mathrm{bp}$, respectively, and determining a premature stop codon in the mutant allele, thus giving raise to a truncated and unfunctional protein. No mutation was detected in patients with isolated PPHP. Conclusion: Here we report the first Italian study on a series of patients with PHP Ia and PPHP and we describe two novel inactivating frame shift mutations in the Gs $\alpha$ gene responsible for the disease. Furthermore, we confirm that these mutations cannot be detected in families with isolated PPHP, suggesting that these forms of AHO are genetically distinct from PHP Ia. 
P3-429 Bone, Ca, PTH and Vitamin D

The Development of the Distal Radius - a Look at the Bone behind the 'Density'

F. Rauch ${ }^{1}$;C. Neu ${ }^{* 1}$; F. Manz ${ }^{* 1}$;E. Schönau ${ }^{1}$

${ }^{1}$ University of Cologne, Cologne, Germany

It is well known that areal bone mineral density (aBMD) of the distal radius increases in healthy children and adolescents. However, the relative contributions of trabecular and cortical bone as well as of changes in bone size are not known at present. In this study we used peripheral quantitative computed tomography (XCT-2000, Stratec Inc., Germany) to determine total volumetric BMD (vBMD-tot), trabecular vBMD (vBMD-trab) and bone cross-sectional area (CSA) at the ' $4 \%$ site' of the distal radius in a group of 371 children and adolescents (186 females, aged 6 to 23 years) as well as 99 adults aged 25 to 40 years ( 80 females). Based on the physiologic fact that cortical vBMD varies very little and using a cylindrical model of the distal radius, aBMD, bone diameter and cortical thickness were estimated from the primary measures. In accordance with studies using dual X-ray absorptiometry we found that between the age of 6 years and adulthood aBMD increases by $79 \%$ and $105 \%$ in females and males, respectively. The changes in aBMD are due to an increase in bone diameter (by $31 \%$ in females and by $47 \%$ in males) and in vBMD-tot (by 37\% in females and by $40 \%$ in males). The increase in vBMD-tot is the effect of an increase in cortical thickness (by $191 \%$ in females and by $212 \%$ in males), whereas vBMD-trab varied little $(5 \%$ decrease in females, $9 \%$ increase in males). Thus, aBMD at the distal radius increases, because bone size and cortical thickness increase. These changes are the result of modeling at the periosteal surface. In conclusion, 'bone mineral accumulation' at the distal radius during growth is the result of modeling by periosteal osteoblasts rather than of endosteal remodeling.

P3-430 Bone, Ca, PTH and Vitamin D

Control Study of Bone Mineral Density in Adults with Childhood Onset Atopic Dermatitis Treated with Topical Glucocorticoids

J.A. Ellison*1; L. Patel*2; T.J. David*2; J.E. Adams*2; M.Z. Mughal*2 Introduced by P.E. Clayton

${ }^{1}$ Leighton Hospital, Cheshire; ${ }^{2}$ Manchester, UK

Bone loss is a serious adverse effect of systemic glucocorticoid (GC) treatment and is thought to be related to the dose and duration of treatment. Topical GCs are the most useful form of treatment for atopic dermatitis (AD) but long term use through childhood may contribute to reduced bone mineralisation. Objective: To determine whether long term percutaneous absorption of topical GC in $\mathrm{AD}$ is associated with adverse effects on bone mineralisation. Patients and Methods: A control study of 19 patients (age 18.2-29.9 years, median 22.6; mean height standard deviation score (SDS) -0.3) with childhood onset AD; 18 healthy adults ( 10 men, 8 women, median age 19.6; mean height SDS 0.2); and 10 adults with asthma but no other illness ( 2 men, 8 women, median age 20.4 ; mean height SDS 0.42) was undertaken. All AD patients had used topical GC for median 20.2 years (4-29.1 years). 17 AD patients also had coexisting asthma and had received inhaled GC for median 10.5 years and 13 of 17 had received up to 6 short courses of oral GC for exacerbations of asthma. The asthmatic subjects had received inhaled GC for median 8.5 years and 7 had received up to 7 short courses of oral GC. Bone mineral density (BMD) was measured by dual-energy X-ray absorptiometry (DEXA) at the lumbar spine and left hip (Hologic QDR-4500 DXA), peripheral quantitative computed tomography (pQCT) of the distal radius (Stratec-Norland XCT 2000) and broadband ultrasound attenuation (BUA) of the left calcaneum (McCue Contact Ultrasound Bone Analyser). SDS were calculated using normal reference data. Comparisons between BMD SDS were made using one-way ANOVA and 2-sample t-test. Results: BMD SDS were significantly reduced at left femoral neck, left hip and right calcaneum in AD group when compared to asthma group (mean -1.19 vs $-0.12,-0.77$ vs $0.15,-1.04$ vs $0.11 ; p$ values $<0.03$ ) and healthy controls (mean -1.19 vs $-0.32,-0.77$ vs $0.22,-1.04$ vs 0.17 ; $p$ values $<$ 0.01 ). In the AD group, lumbar spine BMD SDS were lower than in the asthma group (mean -0.92 vs $0.22 ; p<0.02$ ), and lower in those treated with potent than mild or moderate topical GCs (mean -1.33 vs -0.23 ; $\mathrm{p} \leq 0.03$ ). There were no differences between asthma and healthy controls. BMD was not influenced by diet, exercise, smoking or oral contraceptive pill use. Conclusions: Topical GC treatment with potent preparations during childhood is associated with poor bone mineralisation. This may contribute to reduced peak bone mass, with the attendant risk of earlier onset of osteoporosis in adult life.

\section{P3-431 Bone, Ca, PTH and Vitamin D \\ An Anusual Combination of Pseudohypoparathyroidism (PHP) Type $1 \mathrm{~A}$ and Subaortic Stenosis in a Girl with a de novo Mutation in GNAS1 Gene \\ V.L. Brunelli*1; G. Mantovani ${ }^{2}$; A. Nardini*1; L. Zannini*3; A. Colli ${ }^{1}$; Introduced by L. Gargantini \\ 1 Ospedale Buzzi, ICP, Milano, Italy; ${ }^{2}$ Ospedale Maggiore, Milano, \\ Italy; ${ }^{3}$ Ospedale Gaslini, Genova, Italy}

Introduction: PHP1A is due to mutations in GNAS1 gene-20q13.2-coding for Gs $\alpha$ subunit of GS proteins, coupled with cellular membrane receptors. Parental imprinting (Davies, Hughes, 1993) or a tissue specific imprinting (Yu et al. 1998) seem to be involved in expression of the disease. Subaortic stenosis is usually an isolated cardiac congenital malformation often becoming symptomatic in adolescence or adulthood. It was observed to be transmitted with dominant inheritence in a Turkish family (Onat, 1984, OMIM 271960) as a part of a multisystem disorder with short stature, obstructive lung disease and hoarseness. Genes involved in Onat syndrome (OS) are not yet identified. Case Report: E.C., the first child of unrelated healthy Egyptian parents, was born after an uneventful pregnancy (PN: $3040 \mathrm{~g}$ ). At 4 years of age she underwent surgical excision of a subcutaneous calcification at the right ankle and consequently pediatric evaluation. She had normal intelligence, rounded facies, ipoplastic enamel, brachimetaphalangism, short stature $(20 \mathrm{pct})$ related to the genetic target $(75 \mathrm{pct})$ and an ejection murmur radiating to the neck $(4 / 6)$. Hypocalcemia $(7.4 \mathrm{mg} \%)$, hyperphosphoremia $(7.8 \mathrm{mg} \%)$ and high PTH plasma levels $(263 \mathrm{pg} / \mathrm{ml})$ suggested the diagnosis of PHP. Further investigations excluded ocular and cerebral anomalies. A subaortic stenosis was evident at a cardiac ultrasound (pic gradient $33 \mathrm{~mm} \mathrm{Hg}$ ). Molecular analysis of GNAS1 gene showed a previously described 4-bp deletion in exon 7 in the proband, while no mutation was found in the other members of the family. After 1 year of treatment with $1,25 \mathrm{OHD} 3(20 \mathrm{ng} / \mathrm{kg} / \mathrm{die})$, calcium supplementation $(750 \mathrm{mg} /$ die per os) and hypophosphoremic diet (P:650 mg/die) good control of $\mathrm{Ca} / \mathrm{P}$ metabolism was achieved. Due to rapid progression (pic gradient $70 \mathrm{~mm} \mathrm{Hg}$ ) she was referred to surgery: a typical fibrous ring was excised successfully and no calcication noted. Surgically corrected. Discussion: To our knowledge, the case is the first description of subaortic stenosis in pseudohypoparathyroidism. Is association between PHP and subaortic stenosis casual or could it be explained on a genetic ground? However, as subaortic stenosis can be asymptomatic in childhood, we suggest to consider a clinical cardiac evaluation in children with PHP 
P3-432 Bone, Ca, PTH and Vitamin D

\section{GNAS1 Mutational Analysis in 8 Italian Albright Hereditary Osteodystrophy (AHO) Patients: Identification of $\mathbf{2}$ Novel Mutations}

L. de Sanctis*1; F. Buzi2; G. Scire*3; D. Romagnolo*1; R. Lala*1; C. de Sanctis* 1

${ }^{1}$ University of Turin, Turin, Italy; ${ }^{2}$ University of Brescia, Brescia,

Italy; ${ }^{3}$ University Tor Vergata, Roma, Italy

Gsalpha protein deficiency has been described in patients with Albright hereditary osteodystrophy (AHO), a genetic disorder that comprises two variants, Pseudohypoparathyroidism type Ia (PHP Ia) and Pseudopseudohypoparathyroidism (PPHP). So far several mutations have been identified in GNAS1 gene encoding Gsalpha protein and the same mutation has been found associated with both PHP Ia and PPHP. Objective: To evaluate GNAS1 mutational analysis in 8 Italian AHO patients. Patients and Methods: We studied 8 subjects from 6 unrelated Italian families. Criteria used for the diagnosis of AHO include: short stature, obesity, brachydactyly, subcutaneous ossifications and mental retardation. All patients showed 3-5 AHO signs; 1 patient has only a PTH resistance, whereas 6 patients have PTH, TSH and FSH/LH resistance. Genomic DNA was isolated from leukocytes; PCR amplification of exons 2-13 were performed using GNAS1 specific primers and conditions; the PCR products were directly sequenced. DNA sequence abnormalities were confirmed by restriction analysis. Results: Two new mutations (delC185 and R336W) and a polymorphism ( $\left.{ }^{131} \mathrm{I}\right)$ have been identified in 3 patients. DelC185 is a deletion that causes a frame shift, premature stop codon and it results in a truncated protein. It has been found in the 2 affected kindred and in the PPHP mother. $\mathrm{R} 336 \mathrm{~W}$ is a missense mutation $(\mathrm{C} \rightarrow \mathrm{T})$ in exon 12 that changes Arg to Trp. It has been found in a patient and excluded in both parents. ${ }^{131} \mathrm{I}$ has been found in the same patient and in the mother. Conclusions: GNAS1 mutational analysis allowed to make diagnosis of PHP-Ia in 3 patients. Moreover, these molecular data demonstrate the genetic heterogeneity for Gsalpha protein deficiencies also in the Italian population, confirm the phenotypic variability, as PHP-Ia and PPHP are found within the same family, and suggested genomic imprinting as potencial mechanism to explain the co-existence of both diseases in the same family. The single patient with PTH resistance and the 3 patients with PTH and multi hormone resistance could harbour mutations in exon 1 or in the promoter region of GNAS1 or could be diagnosed as PHP type Ib or Ic. The patient with isolated AHO showed brachydactyly, obesity and mental retardation, features quite nonspecific or present in other disorders (Turner's syndrome, acrodysostosis, or a deletion at $2 \mathrm{q} 37$ ). This study confirms the AHO heterogeneity and evidences the role of molecular analysis to make a definite diagnosis of PHP-Ia and PPHP.

\section{P3-433 Bone, $\mathrm{Ca}$, PTH and Vitamin D}

Idiopathic Hypercalcemia Associated with Eosinophilic Fasciitis

P. Backeljauw ${ }^{* 1} ;$ M. Rutter ${ }^{* 1}$; Introduced by M. Maes

${ }^{1}$ Children's Hospital Medical Center, Cincinnati, Ohio, United States

Background: A previously healthy 15-year old boy developed symmetrical swelling of his wrists and ankles, involving his knees within a month. He had a sensation of 'tightness' and limitation of movement. His milk intake was up to two litres per day ( $2.3 \mathrm{~g}$ calcium/day) and he took a food supplement containing glucosamine chondroitin as well. He had polyuria. Physical Findings: Height and weight were 90th percentile. He appeared anaemic, but was otherwise well, afebrile and normotensive, without rash, nor lymphadenopathy. He had symmetrical pitting edema in a glove-and-stocking distribution. Remaining exam was normal. Investigations: Serum calcium ranged from 11.5 to $12.7 \mathrm{mg} / \mathrm{dl}$, phosphate was $3.3 \mathrm{mg} / \mathrm{dl}$, alkaline phosphatase $67 \mathrm{IU} / 1$. Intact-PTH was $2 \mathrm{pg} /$ $\mathrm{ml}$. PTHrP was $<0.2$ and $0.6 \mathrm{pmol} / \mathrm{l}(\mathrm{nl} .<1.3)$. Serum albumin and total protein were 3.0 and $6.1 \mathrm{~g} / \mathrm{dl}$, respectively. He had mild alkalosis and BUN and creatinine were 30 and $1.4 \mathrm{~g} / \mathrm{dl}$. Creatinine clearance was normal $(103 \mathrm{ml} / \mathrm{min} /$ $\left.1.73 \mathrm{~m}^{2}\right)$, but he had increased urinary calcium excretion $(13.8 \mathrm{mg} / \mathrm{kg} / 24 \mathrm{~h})$ and protein excretion (protein/creatinine ratio 0.38 ). He had normochromic normocytic anaemia (hemoglobin $10.7 \mathrm{~g} / \mathrm{dl}$ ) and elevated eosinophil count $(13 \%)$, but remaining blood count and smear were negative. Sedimentation rate was high $(30 \mathrm{~mm} / \mathrm{h})$. Thyroid tests, cortisol, LDH, and vitamin A and D concentrations were normal. Angiotensin converting enzyme was 52 U/1 (nl. 9-33.4). Aldolase was high (21 IU/l; nl. 2.9-8.6). Long bone radiographs, bone scan, abdominal ultrasound, CT chest/abdomen/pelvis and MRI of the head were negative. Bone marrow biopsy revealed eosinophilic myelosis without evidence of malignancy. MRI of the forearms and biopsy were consistent with eosinophilic fasciitis. Steroid treatment resulted in significant clinical improvement and resolution of the hypercalcemia. Conclusion: An association between eosinophilic fasciitis and hypercalcemia has not been reported previously. The co-existance of an underlying (pre-)malignant condition needs to be considered, despite a negative evaluation. The pathophysiology of this patient's hypercalcemia remains obscure. Possible explanations must include increased cytokine production, such as interleukin-1, -5 or -6 , associated with the underlying inflammatory process of fasciitis.

P3-435 Bone, Ca, PTH and Vitamin D

\section{Prenatal Diagnosis of the New HRD}

(Hypoparathyroidism Retardation and Dysmorphism)

\section{Syndrome}

E. Hershkovitz $^{1} ;$ R. Hershkovitz ${ }^{* 2} ;$ R. Gorodischer ${ }^{* 1}$; L. Hertzug ${ }^{* 1}$; R. Parvari*3.

1 Pediatric Department; ${ }^{2}$ Obstetric and Gynecology Department:

${ }^{3}$ Molecular Genetics Laboratory, Faculty of Health Sciences, Soroka

Medical Center, Ben-Gurion University of the Negev, Beer Sheva,

Israel

The new syndrome of Hypoparathyroidism, growth and mental Retardation, and Dysmorphism (HRD, MIM 241410) has been recently described in patients of Arab origin. We have recently mapped the human HRD gene locus to a $1 \mathrm{cM}$ interval on the long arm of chromosome I (1q42-43) However, the genomic structure of the HRD gene and its product are unknown Objective: Using linkage analysis, we report the first prenatal diagnosis in four unrelated families with HRD. In addition, we describe some prenatal sonographic findings of anaffected fetus with HRD. Patients and Methods: Since the mapping of the HRD gene, four mothers who previously gave birth to at least one affected child were referred with a request for prenatal diagnosis in five ongoing pregnancies. Blood samples were taken from the parents, the affected children and the healthy siblings). Amniocentesis was done at 16-18 weeks of gestation. Results: Five prenatal diagnoses were done using the linked markers on chromosome 1q42-43. D1S235 had a lod score of 4.11 at a recombination fraction of 0 . The use of at least 4 markers including both centromeric and telomeric regions to the HRD map position enabled the segregation analysis of the haplotype of the HRD locus and thus the prediction of the embryo status. Three fetuses were found to be carriers and were subsequently born healthy. The parents of the two affected fetuses were advised about the results but due to religious and cultural reasons one couple preferred not to terminate the pregnancy. Clinical and biochemical studies confirmed the diagnosis in one affected newborn while the other pregnancy has been terminated. No parathyroid gland could be demonstrated in the aborted fetus. Sonographic evaluation of one affected fetus was normal at 12 weeks of gestation. However, significant growth retardation was found at 18 weeks of gestation. The growth retardation was symmetric; head diameters (BPD, OFD) were compatible with 16 weeks of gestation. Abdominal circumference and long bones measurements were similarly retarded. No major malformations were discovered. Conclusion: Linkage analysis by informative markers was useful in making a reliable prenatal diagnosis in families who had previously at least one affected child with HRD. Hopefully, uncovering the gene structure and its product will enable us to provide accurate genetic counseling to the other family members. 
P3-436 Bone, Ca, PTH and Vitamin D

Total Bone Mineral Content throughout Puberty. Dual-Energy X-Ray Absorptiometry Study

D. Yeste ${ }^{1} ;$ N. Garcia Reyna1; L. Del Rio ${ }^{1} ;$ M. Gussinyé1;

M.A. Albisu ${ }^{1} ;$ E. Vicens-Calvet ${ }^{1} ;$ A. Carrascosa ${ }^{1}$

${ }^{1}$ Hospital Universitario Materno-infantil, Barcelona, Spain

Objective: Technologic advances and informatic support in DEXA devices permit study of whole-skeleton bone mineral content and its anatomical distribution pattern in a shorter time and with minimal exposure to ionizing radiation. Bone mass evolution throughout puberty was analysed using this technique in a population of healthy children and adolescents in our area. Subjects and Methods: Cross-sectional study of 116 children and adolescents ( 55 boys and 61 girls) with ages ranging from 7 to 17 years, classified by Tanner stages. Analysis of total bone mineral content (TBMC), expressed as grams (g) of hydroxyapatite, and its distribution pattern in the skeleton (head, trunk, upper and lower limbs) was made by DEXA (Lunar DPX-L, software 1.3y). Results: TBMC of the skeleton increases progressively and statistically significantly in puberty in both sexes. TBMC of boys was $1,567 \mathrm{~g}$ at onset of puberty, reaching Tanner stage $\mathrm{V}$ of $2,544 \mathrm{~g}$ (increment: $62 \%$ ). Girls passed from TBMC of $1,143 \mathrm{~g}$ to $2,018 \mathrm{~g}$ in the final stages (increment: $76 \%$ ). The increase in TBMC in girls was produced progressively and staggered throughout puberty, whereas in boys statistically-significant increments were produced between Tanner stages II and III and between III and IV. The percentage of TBMC contributed by each anatomical region to the TBMC of the skeleton showed significant variations with the progression of puberty. TBMC of the skull remained practically invariable, while significant increases in trunk and limbs were observed in both sexes. Mineral salt deposits in the skeleton per centimetre of height gain in puberty was identical in both sexes (35.2 g hydroxyapatite/cm height). Conclusions: Significant variations in acquisition rate and anatomic distribution of skeletal mineral salt deposit are produced in puberty. The bone mass acquisition pattern during puberty appears to show a sexual dimorphism.

\section{P3-437 Bone, Ca, PTH and Vitamin D \\ Reduced Bone Mineral Density in Patients with \\ Thalassemia major: Comparison between Spontaneous and Hormonally Induced Puberty \\ M. Cisternino ${ }^{1}$; L. Bassi*1; P. Comoli*1 1; M. Baldi*2 ; \\ O. Tomarchio *2; M. Zecca*1 \\ ${ }^{1}$ University of Pavia, Pavia, Italy; ${ }^{2}$ IRCCS Fondazione Medicina del \\ Lavoro, Pavia, Italy}

A low bone mineral density (BMD) mainly due to endocrine complications has been reported in patients affected by thalassemia major. The aim of this study was to evaluate the BMD in thalassemic patients and to compare the bone density of patients with spontaneous puberty with that of patients affected by hypogonadism treated with hormonal replacement therapy (HRT). A cohort of 33 transfusion-dependent patients $(14 \mathrm{~F}, 19 \mathrm{M}$, aged $13.5-35$ years) received spinal BMD scan. Ten patients $(6 \mathrm{~F}, 4 \mathrm{M})$ had a spontaneous puberty followed by normal hormonal gonadal production, the remaining 23 patients $(8 \mathrm{~F}, 15 \mathrm{M})$ had either an arrest of the spontaneous puberty or a lack of puberty and therefore have been treated with HRT (HCG/HU-FSH or testosterone in males and transdermal estradiol plus oral medroxyprogesterone in females). In 14/33 patients $(6 \mathrm{~F}, 8 \mathrm{M})(42 \%)$ a severely low bone mass $(\mathrm{Z}$ score $<-2.5)$ and in other $14 / 33(7 \mathrm{~F}, 7 \mathrm{M})(42 \%)$ cases a low bone mass ( $Z$ score between -1 and -2.5 ) was found, only the remaining 5 cases $(4 \mathrm{M}, 1 \mathrm{~F})(15 \%)$ had a normal $\mathrm{Z}$ score $(-1)$. The mean $\mathrm{Z}$ score of females tended to be lower, but not significantly, than that of males (F: $-2.44 \pm 0.95$ vs M: $-1.94 \pm 1.33$ ). The mean BMD of patients with spontaneous puberty was not significantly different from that of hypogonadal treated ones in both sexes (M:-1.23 \pm 1.64 vs $-2.13 \pm 1.23 ; \mathrm{F}:-2.5 \pm$ 0.87 vs $-2.39 \pm 1.06$ ). One out of 5 patients with normal BMD had a spontaneous puberty, the remaining 4 were receiving HRT. No correlations were found between the patients $\mathrm{Z}$ score, the ferritin levels and the hepatic liver enzymes. In conclusion, an impaired BMD has been found in $85 \%$ of patients affected by thalassemia major. The bone mass loss was not significantly different in patients with normal gonadal function with respect to those with hypogonadism treated with HRT. This finding may suggest that the HRT may prevent a further bone mass loss due to the hygonadism in thalassemia major.
P3-438 Bone, Ca, PTH and Vitamin D

\section{Evaluation of Two Different Bisphosphonates in the} Treatment of Osteoporosis in Childhood

S. Bertelloni ${ }^{1}$; G.I. Baroncelli*1; S. Navari*1; G. Saggese ${ }^{1}$

${ }^{1}$ Santa Chiara Hospital, Pisa, Italy

Background: No therapy is universally accepted for osteoporosis in childhood. Bisphosphonates may be of value, but the paediatric experience has been limited. Objective: To evaluate the effects on bone mineral density (BMD) of two bisphosphonates in paediatric patients with osteoporosis. Patients and Methods: Eleven patients (mean age $14.9 \pm 3.7$ years; 4 males) with severe osteoporosis (areal BMD $<-2.5$ SDS for age and sex) were studied; bone fractures or vertebral collapses were present in 5 subjects and bone pains in 9 . Diagnoses were: idiopathic juvenile osteoporosis $(n=2)$, osteogenesis imperfecta $(n=2)$; steroid induced $(n=2)$; survivor of paediatric malignancy $(n=4)$, dysmorphic syndrome $(\mathrm{n}=1)$. The patients were treated by pamidronate (PM: $0.5-1.0 \mathrm{mg} / \mathrm{kg}$ i. $\mathrm{v}$. for 3 days every $4-6$ months; $\mathrm{n}=6$ ) or alendronate (AL: $10 \mathrm{mg} /$ daily; $\mathrm{n}=5$ ). Areal BMD (aBMD) was measured at lumbar spine (L2L4; Lunar DPX) and volumetric BMD (vBMD) was calculated by Kroger's formula (Bone Miner, 1993). Data are in SDS according to age and sex. Results: aBMD and vBMD significantly increased $(\mathrm{p}<0.02)$ in both PM and AL treated patients; no difference was present at 1 year follow-up between the groups (table). No patient had additional fractures.

\begin{tabular}{llllll}
\hline Months & 0 & & & 12 & \\
\cline { 2 - 3 } \cline { 5 - 6 } & aBMD & vBMD & & aBMD & vBMD \\
\hline PM & $-4.5 \pm 1.2$ & $-4.9 \pm 3.1$ & & $-1.8 \pm 0.9$ & $-1.4 \pm 1.1$ \\
AL & $-5.0 \pm 1.6$ & $-3.1 \pm 1.1$ & & $-1.5 \pm 1.2$ & $-0.9 \pm 1.2$ \\
\hline
\end{tabular}

Bone pains recovered. No important side effects occurred. Conclusions: Bisphosphonates are able to increase BMD in paediatric patients with osteoporosis from different aetiologies and to improve disease-related symptoms PM and AL seem to be equally effective.

P3-439 Bone, Ca, PTH and Vitamin D

\section{Late Catch-Up of Bone Strength in Prematurely-Born Children. Preliminary Results}

Z. Zadik ${ }^{1}$; A. Shamaev*2; D. Geva*2

${ }^{1}$ Kapla Medical Center, Rehovot, Israel; ${ }^{2}$ Sunlight Ultrasound

Technologies Ltd., Rehovot, Israel

Premature-born infants (PBI) are prone for developing osteopenia. In order to follow the extent of osteopenia and the recovery process. The standard methods for bone density (BD) determinations are based on X ray irradiation. Most of the prematures were exposed to numerous $\mathrm{X}$ rays. In addition to that $\mathrm{BD}$ technics utilizing $X$ ray can not be used in a regular office or at the bedside. To prevent additional $X$ ray exposure we evaluated a new system that uses ultrasound. BD was measured by the Sunlight Omnisense (Omnisense), a QUS device designed to measure SOS at multiple skeletal sites by axial transmission method. 504 children (197 male and 307 females) were studied. Twenty-two were PBI (14 male and 8 female) and 482 sex- and age-matched children served as controls. Age range was $1-8$ years (mean age $4.2 \pm 2.0$ ). Participants were measured at the Mid-Shaft Tibia by the Omnisense. The measured SOS of the PBI group was found to be lower than that of full-term born infants. The catchup of bone mineralization continued until the age of 6 in the PBI, while older children seem to keep to the normal curve. Conclusion: It seems that the diminished BD noted in premature infants may last up to 6 years. Additional studies in these children are needed in order to investigate the metabolic basis of the reduced BD. The Omnisense which is devoid of $\mathrm{X}$ ray irradiation and known biohazards, easy to operate, is an ideal device for following BD changes in premature born infants. 
P3-440 Bone, Ca, PTH and Vitamin D

Results of Bone Analysis in Adolescents Suffering from Anorexia nervosa

O. Fricke ${ }^{* 1} ;$ B. Tutlewski*1;E. Schönau ${ }^{1}$

${ }^{1}$ University of Cologne, Cologne, Germany

Influence of anorexia nervosa to the muscle-bone interaction is unknown. Objective: To analyse the muscle-bone interaction in female adolescents who suffered from anorexia nervosa. Patients and Methods: In a retrospective study 25 female adolescents who had suffered from anorexia nervosa and who had been admitted to our hospital more than 3 years ago were analysed for bone density, for bone geometry, for parameters of the calcium-phosphor homoeostasis and for parameters of the muscle-bone interaction. Peripheral quantitative CT and an instrument for measuring muscle strength were used for the analysis of bone and muscle at the forearm. Results: $20 \%$ of the adolescents had a BMI lower than $17.5 \mathrm{~kg} / \mathrm{m}$. The bone density was decreased (total bone density $\mathrm{T}=-1.55, \mathrm{Z}=-1.38$, trabecular bone density $\mathrm{T}=-0.78, \mathrm{Z}=-0.66$ ). The correlation between bone strength (SSI) and muscle force was high significant (distal $r=0.41$, proximal $r=0.44$ ). Furthermore the correlation between bone strength and BMI was high significant $(\mathrm{r}=0.66)$. All patients had normal values for parameters of the calcium-phosphor homoeostasis. Conclusions: Adolescents have also a decreased bone mass a couple of years after the treatment of anorexia nervosa. In comparison to the normal adolescents the females who suffered from anorexia nervosa have a decreased correlation between bone strength and muscle force. Maybe the lack of estrogen in anorectic females reduces the sensitivity of bone for mechanical signals.

P3-441 Bone, Ca, PTH and Vitamin D

\section{Hypocalcemia and Hypoparathyroidism in Children with 22q11.2 Deletion Syndrome}

A. de Gonneville*1; C. Kindermans*1; J. Lebidois*1; S. Lyonnet*1.

M. Prieur ${ }^{* 1} ;$ R. Brauner ${ }^{1}$

1 Hôpital Necker-Enfants Malades, Paris, France

The aim of this study was to evaluate the prevalence of hypocalcemia and hypoparathyroidism in children with $22 \mathrm{q} 11.2$ deletion syndromes. A cross-sectional study was performed over a 14-month period, in 39 children ( 20 girls) at a mean age of 9.7 years $(2.5-20)$. The initial symptoms were: cardiac defect $(n=$ $29)$, facial dymorphism $(n=6)$, seizures $(n=7)$ and/or hypoplasia of the thymus $(\mathrm{n}=6)$. Serum biochemical tests (serum ionized and total calcium, phosphatemia, intact PTH) were performed by means of standard laboratory methods. Seven children ( $18 \%$ ) presented hypocalcemia and hyperphosphatemia associated with low $(n=2)$ or inappropriately low-normal $(n=5)$ PTH. Ten children $(25 \%)$ presented low-normal calcemia with inappropriately low normal PTH. Among these 10 patients, only 2 had transiently low PTH at birth. We thus found a high prevalence (43\%) of biological feature of hypoparathyroidism in children with $22 \mathrm{q} 11.2$ deletion syndromes. It is must be stressed that in 10 out of these 17 patients with a biological hypoparathyroidism, there was no clinical symptom of hypocalcemia previously reported. Conclusion: Our results suggest that children with 22q 11.2 deletion syndromes are at risk of clinical symptoms of hypoparathyroidism. This risk may be enhanced in case of previous hypocalcemia, surgical stress or other stresses. We suggest that calcium PTH axis must be carefully assessed during the medical follow-up of these patients.

P3-442 Bone, Ca, PTH and Vitamin D

\section{Clinical and Genetic Heterogeneity in Two Patients with Hypophosphatasia}

M. Hauschild ${ }^{* 1} ;$ D. Schnabel ${ }^{* 2}$; E. Mornet ${ }^{* 3} ;$ P. Freisinger ${ }^{* 4}$;

A. Grüters ${ }^{2} ;$ P.H. Heidemann ${ }^{1}$

${ }^{1}$ Zentralklinikum, Augsburg, Germany; ${ }^{2}$ Universitätsklinikum

Charité, Berlin, Germany; ${ }^{3}$ Université de Versailles, Versailles,

France; ${ }^{4}$ Technische Universität, München, Germany

Introduction: Hypophosphatasia is a rare inherited disorder with decreased activity of alkaline phosphatase leading to deficient ossification of the skeleton.
Mutations in the tissue-nonspecific alkaline phosphatase gene are associated with a variable phenotypic expression ranging from congemital lethal form to the milder hypophosphatasia tarda. The factors leading to this heterogeneity are not known. Patients: Pat. 1 was diagnosed at the age of $2 \frac{1 / 2}{2}$ months with muscular hypotonia, feeding problems, dystrophy $(3980 \mathrm{~g})$ and wide fontanels. Laboratory and radiologic results revealed hypophosphatasia (ALP $61 \mathrm{U} / 1$; normal 145-420), hypercalcemia $(4.06 \mathrm{mmol} / \mathrm{l})$, hypercalciuria (calcium-creatinine-ratio: $4.1 \mathrm{mg} / \mathrm{mg}$ ), nephrocalcinosis, severe bone demineralisation and multiple fractures. Experimental therapy with calcitonin, hydrochlorothiazide and pamidronate could not influence ossification. He died at the age of 13 months. Screening for mutations revealed compound heterozygousity with maternal missense mutation M45L and paternal single nucleotide deletion $1172 \mathrm{delC}$ leading to a stop codon. Pat. 2 presented at the age of 11 months with growth retardation, a wide anterior fontanelle and painful movements of both legs and arms. Diagnostics revealed hypophosphatasia (65 U/1), normocalcemia $(2.5 \mathrm{mmol} / \mathrm{l})$ normocalciuria (ratio: $0.21 \mathrm{mg} / \mathrm{mg}$ ) and severe bone demineralisation. Without specific therapy he achieved a catch-up growth and normal development until the age of presently three years. Screening for mutations revealed compound heterozygousity with maternal A23V and paternal G232V missense mutations. Discussion: Both A23V and G232V mutations were previously found in patients with lethal hypophosphatasia. However, G232V seems to allow residual activity (around 15\%). The 1172 del C mutation is thought to result in the absence of any enzymatic activity, but M45L may allow residual ALP activity. The distinct course of the two patients, both carrying potentially lethal mutations raises the questions of the existence of modifier genes and the role of other factors involved in bone mineralisation.

P3-443 Bone, $\mathrm{Ca}$, PTH and Vitamin D

Variable Calcium Status in Partial Di George Syndrome C. Jeandel ${ }^{* 1}$; V. Sadauskaite*1;P. Sarda*2; M. Voisin ${ }^{* 1}$; J. Astruc*2; R. Dumas ${ }^{* 1} ;$ Ch. Sultan ${ }^{1}$

1 Kaunas University Clinic, Kaunas, Lithuania; ${ }^{2}$ Hôpital

A. de Villeneuve, Montpellier, France

Introduction: Di George Syndrome (DGS) or sequence is characterised by the variable association of hypocalcemia, craniofacial anomalies, cardiac defects, mental disability, growth retardation and abnormal $\mathrm{T}$ and $\mathrm{B}$ cell function. Affected individuals have an interstitial deletion of chromosome 22 q11. Although hypocalcemia is a cardinal symptom of the DGS, it is not always decreased at time of diagnosis. Aim: This work was designed to evaluate the frequency and the age of onset of hypocalcemia in patients with DGS confirmed by 22q11del. Patients and Results: Eighteen children ( 3 newborn, 4 infants, 11 children) with DGS were studied. As expected, craniofacial anomalies were detected in $17 / 18$ cases, cardiac defects were present in $15 / 18$ cases, mental disability in 8112 cases and growth retardation in $7 / 18$ cases. Dysimmunity was present in $7 / 18$. Hypocalcemia was noted in 8 children: calcium status is reported on the following table:

\begin{tabular}{|c|c|c|c|c|c|c|c|}
\hline \multirow[t]{2}{*}{ Case } & \multirow{2}{*}{$\begin{array}{l}\text { Age/ } \\
\text { diagnostic }\end{array}$} & \multicolumn{3}{|c|}{ Hypocalcemia } & \multirow{2}{*}{$\begin{array}{l}\text { Craniof. } \\
\text { anomal. }\end{array}$} & \multirow{2}{*}{$\begin{array}{l}\text { Cardiac } \\
\text { defect }\end{array}$} & \multirow{2}{*}{$\begin{array}{l}\text { Dys- } \\
\text { immun. }\end{array}$} \\
\hline & & neon & trans & perman. & & & \\
\hline 1 & 3 days & + & + & - & + & + & - \\
\hline 2 & 4 days & 1 & 1 & - & + & + & - \\
\hline 3 & 6 years & + & - & + & + & - & - \\
\hline 4 & 3 days & + & - & - & + & + & $?$ \\
\hline 5 & 1 month & + & + & - & + & - & + \\
\hline 6 & 11 years & + & + & - & + & + & - \\
\hline 7 & 9 months & + & + & - & + & - & + \\
\hline 8 & 1 month & + & + & - & + & + & + \\
\hline
\end{tabular}

Conclusion: The analysis of 18 cases point out the large spectrum of presentation of DGS, from cases where the most prominant feature of syndrome is hypocalcemia to cases with asymptomatic latent or late-onset hypocalcemia. We propose to systematically include serum calcium in the care of patients with DGS especially during infancy, adolescence and even during potential pregnancy. 
P3-444 Bone, Ca, PTH and Vitamin D

\section{Athletic Role, Puberty and Bone Assessment in Elite} Adolescent Soccer Players

A. Marchi*1,2;P. Baiardi*3; R. Tavana*4; G. Levizzani*4;

M. Bozzola 1 ; L. Cuzzone ${ }^{* 2}$; L. Bacchella*3; A. Pisati*3.

O. Tomarchio*3

${ }^{1}$ Policlinico S. Matteo; ${ }^{2}$ Sports Medicine Center, ${ }^{3}$ University of

Pavia, Pavia, Italy; ${ }^{4}$ AC Milan Medical Team, Milan, Italy

Modality, intensity and duration of physical exercise are the essential components of the effects of exercise itself on bone assessment, particularly in pubertal age. To evaluate the relationship between puberty and bone metabolism in young athletes, a team of 69 male elite soccer players ( 14 to 16 years), training 3 times a week for 6 years, was tested at two agonistic steps (out and in training) as regards: serum Ca, alkaline phosphatase (AP), 1,25OH-D, 25OH-D, BGP, lumbar spine BMD and BMC (out training), androstenedione (A), DHEAS, free testosterone/cortisol ratio (FT/C), PRL and ACTH. Differences among different athletic roles (10 goal-keepers (GK), 18 full-backs (FB), 22 centre-backs (CB) and 19 forwards (FW)) are stressed. On the whole team, a reduction in AP $(-7.5 \%, \mathrm{p}=0.04)$ and an increase in PRL $(+28 \%, \mathrm{p}<0.001)$ were observed, testimonial of a normal growth despite physical stress. Bone evaluation showed an increase in $1,25 \mathrm{OH}-\mathrm{D}(+10.5 \%, \mathrm{p}<0.05)$, unchanged values for $\mathrm{Ca} / \mathrm{P}$ ratio $(\cong 2.5)$ and good levels of BMD and BMC: evidence of an exercise optimal bout. Moreover, a lucky balance between anabolic and catabolic metabolism was pointed out by FT/C ratio (not significant changes from out to in). Subgroup analysis according to athletic role confirmed the increase in PRL as well as the reduction in AP for all the playing roles. Opposite trends in 1,25OH-D for $\mathrm{CB}$ (steady) and in $\mathrm{FT} / \mathrm{C}$ ratio for GK (decreasing) were respectively observed. $\mathrm{Ca} / \mathrm{P}$ ratio, always unchanged, but higher for $\mathrm{FW}(\cong 2.7)$ and lower for $\mathrm{GK}(\cong 2.3)$. $\mathrm{CB}$ opposite course was balanced by a FT/C ratio slowly increasing, while GK behavior, comparable to FW one, may be due to the exasperation of their athletic tasks (extreme defender compared to great striker). They present an incomplete recovery, a prevailing catabolism probably linked to exercise and pubertal stresses, despite a good bone status. Periodization of hormonal monitoring of training (FT and C) and a contemporary bone and growth speed parameter evaluation in sports adolescents allow to prevent dangerous overtraining.

P3-445 Bone, Ca, PTH and Vitamin D

Type I Collagen N-Telopeptide Changes in Adolescents during Treatment with Isotretinoin for Severe Acne vulgaris

G. Trifiro ${ }^{1}$; M. Bevilacqua ${ }^{1}$; G. Norbiato ${ }^{1}$

${ }^{1}$ University Hospital L. Sacco, Milan, Italy

Isotretinoin, a synthetic 13-cis-retinoic acid, is highly effective in treatment of severe acne vulgaris, but a prolonged use of retinoids has been associated with significant skeletal toxic effects and changes of bone biochemical markers. Among collagen markers, type I collagen N-telopeptide (NTx) has never been studied. NTx is present in all tissues that contain type I collagen, mostly in bone but also in cutaneous tissue. Aim of our study was to evaluate the effects of oral isotretinoin on bone remodeling markers in 10 adolescents (4 males and 6 females, mean age 17.8 years), treated for severe nodulo-cystic acne, resistent to conventional therapy. Patients received a dose of $0.5 \mathrm{mg} / \mathrm{kg} /$ day for three months. We measured urinary NTx as bone resorption marker, alkaline phosphatase (ALP) and osteocalcin (OC) as markers of bone formation, parathyroid hormone $(\mathrm{PTH})$ and metabolites of vitamin $\mathrm{D}$ as $25-\mathrm{OH}-\mathrm{D}_{3}$ and 1,25 $(\mathrm{OH})_{2} \mathrm{D}_{3}$. Determinations were performed before and after isotretinoin treatment. Results: No significant variations were observed in serum ALP, OC, calcium, PTH nor in vitamin D metabolites. A significant decrease of urinary NTx was found $(831.4 \pm 145$ vs $502.9 \pm 56 \mathrm{nmol} \mathrm{BCE} / 24 \mathrm{~h}, \mathrm{p}<0.05)$. Conclusions: The significant decrease of NTx paralleled the clinical remission of acne, thus suggesting that the variation is related to the cutaneous component of type I collagen and not to bone. In dermatologic diseases assessment of NTx could provide informations about skin collagen.
P3-446 Bone, Ca, PTH and Vitamin D

Bone Mineral Status in Adults with Cystic Fibrosis J. De Schepper ${ }^{1}$; S. De Man*1;I. Dab*1;A. Malfroot*1;

Y. Vandenplas ${ }^{*} ; O$. Louis ${ }^{* 2}$

Departments of ${ }^{1}$ Pediatrics and ${ }^{2}$ Radiology, Academic Hospital

VUB, Brussels, Belgium

Impaired skeletal growth and mineralisation have been described previously in children with cystic fibrosis (CF). Objective: To evaluate whether appendicular bone mineral in CF normalizes at end of growth and to look for potential nutritional, metabolic and clinical risk factors for osteopenia at the peripheral skeleton at an adult age in CF. Patients and Methods: Total bone density (BD), trabecular bone density (tBD) and cortical-subcortical bone density (cBD) were measured by peripheral quantitative computed tomography at the distal radius in 25 ( 10 males and 15 females) non selected adult CF patients with a mean age of $27 \pm 6$ years and with a normal height. Results for BD and tBD were also expressed as z-score for age. Nutritional status ( BMI z-score, serum vitamin $\mathrm{E}$, vitamin A, albumin, IGF-1), vitamin D status ( serum 25OHvit D, alcaline phosphatase, calcium and phosphorus), respiratory status (forced vital capacity), liver status (liver ultrasound) and glucose metabolism (fasting glycemia, HbAlc) were analyzed at the same time. Results: Mean BD was $382 \pm 94$, mean tBD $195 \pm 94$ and mean cBD $533 \pm 132 \mathrm{mg} / \mathrm{cm}^{3}$. Mean tBD z-score was $-0.41 \pm 1.47$ with 4 patients presenting values below $-2 \mathrm{SD}$. No sex difference was found in the studied bone mineral parameters. $\mathrm{cBD}$ was correlated with $\operatorname{tBD}(\mathrm{r}=0.69 ; \mathrm{p}=0.0001)$ and age $(\mathrm{r}=0.47 ; \mathrm{p}=0.01)$. BD $\mathrm{z}$-score as well as tBD z-score were positively correlated with vit $\mathrm{E}$ and negatively with alcaline phoshatase levels. $\mathrm{CBD}$ results were lowest in patients with ultrasound signs of cirrhosis (mean value: $333 \pm 21 \mathrm{mg} / \mathrm{cm}^{3}$ ). Conclusions: A decreased trabecular bone density at the radius was found in $16 \%$ of CF adults. The lowest bone mineral values for age can be expected in the patients with the most important fat malabsorption, reflected by a decreased vitamin E level. Cortical bone density appears still increasing after the age of 20 years. CF patients with liver cirrhosis, presenting the highest alcaline phosphatase levels, appear at greatest risk for a decreased cortical density of their peripheral skeleton.

P3-447 Bone, Ca, PTH and Vitamin D

Intravenous Bisphosphonate Treatment in Osteogenesis imperfecta

D. Göksen*1; M. Coker*1; S. Kara*2; S. Darcan*1

Departments of ${ }^{1}$ Pediatric Endocrinology and Metabolism and

2 Orthopedics, Ege University School of Medicine, Izmir, Turkey

Osteogenesis imperfecta is a disorder characterized by osteopenia, frequent fractures, progressive deformity loss of mobility and chronic bone pain. Objective: To assess the effects of pamidronate on bone resorption. Patients and Methods: In an uncontrolled study involving six children (4 female, 2 male) ages ranging from 1.5 years to 8.5 years (mean $5.1 \pm 3.18$ years) pamidronate was administered intravenously $20 \mathrm{mg} /$ body surface area monthly. Calcium and vitamin $\mathrm{D}$ were administered in appropriate doses according to the serum levels. Clinical status, biochemical characteristics, bone mineral density of the lumbar spine on L2-L4 vertebra and radiological changes were assessed on the sixth month. Results: Administration of pamidronate resulted in a reduction in serum ALP levels from $650 \pm 201$ to $452 \pm 99$ on the sixth month. BMD values increased from $0.200 \pm 068 \mathrm{~g} / \mathrm{cm}^{2}$ to $0.290 \pm 0.010 \mathrm{~g} / \mathrm{cm}^{2}$ with an increase of $45 \%$ on the sixth month. Treatment with parnidronate altered the rate of fractures from 4-5 to none annually. Mobility and ambulation improved in all of the patients shown with a scoring system. Conclusion: Cyclic administration of intravenous pamidronate with calcium and vitamin D supportive therapy improved clinical outcomes, reduced bone resorption and increased bone density. 
P2-448 Fat Metabolism and Obesity

\section{Obesity and Alterations of Cardiac Rhythm Regulation} in Children

A.P Averianov*1; N.V. Bolotova*1; E.V. Panfilova*1

Department of Pediatric Endocrinology, State Medical University,

Saratov, Russian Federation

The issue of autonomic disorders in children with obesity is still to be clarified. Application of heart rate variability analysis (HRV) to evaluate the status of autonomic nervous regulation appears to be of practical interest. Objective: To determine the nature of autonomic disorders and regulatory alterations of cardiac rhythm in obesity at the pediatric age. Patients and Methods: 64 children aged 9-15 were examined. All of them underwent HRV. The children were divided into 2 groups on the basis of the diagnosis. Group 1 included 23 patients, suffering from alimentary-constitutional obesity (ACO). Group 2 comprised 41 patients with complicated obesity -hypothalamic syndrome (HS). Results: The children with HS showed predominance of III class of obesity, the peripheral symptoms of autonomous disorders such as hyperhydrosis, "marble' skin, acrocyanosis. According to HRV increased sympathetic status and activity of dyencephalic nervous centers were observed in $78 \%$ children with HS vs $17.4 \%$ in ACO. Hypersympathetic response to standing-up test was registered more often in HS, which testified to regulatory mechanisms strain. HRV analysis in accordance with BMI revealed that in children with III class of obesity increase of cardiac rhythm disorders such as intensive arrythmia, rhythm stabilization $(\mathrm{dRR}<0.10 \mathrm{sec})$ were registered more often, while cases of balanced autonomic state were less common. Conclusions: Disorders of autonomic homeostasis are common in children with complicated obesity. The prevalent type of autonomic tone is sympathetic one. The increase of BMI correlates with the intensity of the regulatory cardiac rhythm alterations.

P2-449 Fat Metabolism and Obesity

Lipoprotein a (LpA) in Obese Children with Familial Risk of Cardiovascular Disease (CVD)

N. Cabrinety*1; M.J. Pisonero*1;A. Armenteras*1; J.M. Borras*1.

J. Ajram*1;J.M. Cuatrecasas ${ }^{2}$

${ }^{1}$ Hospital Sagrat Cor., Barcelona, Spain; ${ }^{2}$ C.M. Teknon, Barcelona Spain

The Lipoprotein A (LpA) has close relationship with low density lipoproteins (LDL). Levels up to $30 \mathrm{mg} / \mathrm{dl}$ indicate high risk of CVD. Its relationship with infantile obesity and familial prevalence seem to be evident. Objective: To evaluate the relationship between $\mathrm{LpA}$ levels with familial prevalence of CVD and infantile obesity. Patients and Methods: Levels of LpA in 837 prepubertal obese children (442 girls and 395 boys) aged from 6 to 8 years, were determined after two days with low fat diet and after 12 hours with no food intake. The familial risk of CVD was evaluated by complete anamnesis of the relatives aged less than 50 years. The LpA levels were determined by nephelometry technique. Student $t$ was used for statistic signification between groups. Man-Whitney test was used for non parametric values. For variable correlation ratio Spearman rank test was employed. Results:

\begin{tabular}{lcl}
\hline Obese BMI & $>30\left(\mathrm{~kg} / \mathrm{m}^{2}\right)$ & \\
LpA $(\mathrm{mg} / \mathrm{dl})$ & $27.01 \pm 0.7$ & Fathers with CVD \\
& $32.07 \pm 1.2$ & Fathers and Grandfathers CVD \\
& $17.12 \pm 0.1$ & No familial CVD \\
Obese BMI & $<30\left(\mathrm{~kg} / \mathrm{m}^{2}\right)$ & \\
LpA $(\mathrm{mg} / \mathrm{dl})$ & $21.19 \pm 1.2$ & Fathers with CVD \\
& $27.07 \pm 0.8$ & Fathers and Grandfathers CVD \\
& $15.19 \pm 1.5$ & No familial CVD \\
\hline
\end{tabular}

Conclusions: The high levels of LpA are significatively elevated $(p<0.01)$ in those cases of infantile obesity with very high familial CVD prevalence.
P2-450 Fat Metabolism and Obesity

Early Onset of Type 2 Diabetes in an Obese Paediatric Population

A. Drake*1;L. Greenhalgh*1;R. Newbury-Ecob*1;I.S. Farooqi*2; J.P.H. Shield*1; E.C. Crowne ${ }^{1}$

1 The Royal Hospital for Sick Children, Bristol, United Kingdom;

2 University of Cambridge, Cambridge, United Kingdom

The rapidly increasing prevalence of obesity in adults is now being reflected in the childhood population. Initial published studies report on children from minority populations with an established increased incidence of non-insulin dependent diabetes mellitus (NIDDM). There are few data in children from non-minority populations concerning the increasing prevalence of obesity, and the potential increased risk of insulin resistance and NIDDM. A regional multidisciplinary paediatric obesity clinic has been established and we present a cross sectional study of the results of patients referred with pathological obesity (BMI $>2 \mathrm{SD})$ during the first year. All patients had height, weight and blood pressure measurements and were seen and assessed by clinicians with interests in clinical genetics, metabolism and endocrinology. Fasting blood was sent for insulin, proinsulin, split proinsulin and leptin. Results: 36 patients with prepubertal onset of pathological obesity were assessed, (17 males; 19 females; age range $3.6-17.9$ years; 17 prepubertal). The median (range) of BMI standard deviation score was $3.47 \mathrm{~kg} / \mathrm{m}^{2}(2.04-6.36)$. 13/36 children had both systolic and diastolic blood pressures above the 90 th centile for age and sex. Dysmorphic features were noted in eight individuals, five of whom had a recognisable syndrome or cytogenetic abnormality. Learning difficulties were present in 14 children. 4/17 of the prepubertal children had an elevated fasting insulin level $(60<\mathrm{pmol} / \mathrm{l})$ and $7 / 17 \mathrm{had}$ elevated proinsulin levels $(>5 \mathrm{pmol} / \mathrm{l})$. Of the pubertal children, $7 / 19$ had fasting insulin levels $>120 \mathrm{pmol} / 1$ and $14 / 19$ had elevated proinsulin levels. One had already developed frank glucose intolerance. Conclusions: This pilot study demonstrates that a significant number of severely obese children have evidence of fasting hyperinsulinaemia, pancreatic dysfunction and hypertension. They are at risk for the early development of NIDDM and its complications and clearly need assessment and management. A number of children seen had syndromic obesity. Longitudinal studies are required to assess the long-term risk factors in these children and the effectiveness of any active intervention.

P2-451 Fat Metabolism and Obesity

\section{Eating Behavior of Children with Simple Obesity}

G. Massa ${ }^{1}$; A. Vanoppen*2; P. Gillis*1; P. Aerssens*1; M. Raes*1; P. Alliet ${ }^{* 1}$

Departments of ${ }^{1}$ Pediatrics and ${ }^{2}$ Nutrition, Virga Jesseziekenhuis, Hasselt, Belgium

Aim Obesity mostly results from a net positive energy balance (energy intake $>$ energy expenditure). The role of macronutrient (fat, protein, carbohydrates) composition of the diet in the development and maintenance of obesity remains unclear. The purpose of this study was to investigate the relationship between body weight and caloric and macronutrient intake in obese children. Subjects and Methods: Forty-five children ( 23 boys) with simple obesity were included. Weight was expressed as relative weight (RW), calculated as the percentage of the ideal body weight (IBW = median weight for height, Dutch references, Roede \& van Wieringen, 1985). Dietary intake was assessed from a 7-day self-reported diet record. Total energy intake and macronutrient composition was calculated with the Becel Nutrition Program (vs. 5.0) and compared with age and gender specific norms. As the norms do not take into account weight and height of the subjects, caloric intake was corrected for the overweight as follows: caloric intake/RW $\times$ 100. Results: Mean \pm SD age of the subjects was $9.8 \pm 2.4$ years. Relative weight was $158 \pm 22 \%$ of IBW (boys $168 \pm 18 \%$; girls $148 \pm 23 \% ; \mathrm{p}<0.05$ ). Daily caloric intake was $114 \pm 18 \%$ (range: $89-169 \%$ ) of the norm $(\mathrm{p}<0.0005)$. Only eighteen children $(40 \%)$ had a daily caloric intake $110 \%$ of the norm. The caloric intake expressed as $\%$ of the norm, was positively related to RW ( $\mathrm{r}=0.52 ; \mathrm{p}<0.0005)$. When the caloric intake was corrected for the overweight, it was $73 \pm 11 \%$ of the norm $(\mathrm{p}<0.0001)$ and it was inversely related to RW $(\mathrm{r}=-0.45 ; \mathrm{p}<0.002)$. Compared to the age and gender specific guidelines the food of the obese children contained more proteins $(13.5 \pm 2.2 \%$ vs $10.9 \%$; $p<0.0001)$, more fat $(38.4 \pm 4.9 \%$ vs $34.0 \% ; \mathrm{p}<0.0001)$ and less carbohydrates $(48.0 \pm 4.8 \%$ vs $54.8 \% ; \mathrm{p}<0.0001)$. There was a strong inverse relationship between the fat and carbohydrate content of the food $(r=-0.90 ; p<0.0001)$. Conclusion: These data suggest that the daily caloric intake of most obese children is not excessively high, especially when the overweight is taken into account. The macronutrient composition of the food with a too high fat and protein content may contribute independently of total energy intake to childhood obesity. 
P2-452 Fat Metabolism and Obesity

$11 \beta$-Hydroxysteroid Dehydrogenase (11 HSD) Activity and Body Composition in Hypothalamic Obesity (HyOb)

D. Tiosano ${ }^{1}$; I. Isenstadt ${ }^{1}$; D. Militiano ${ }^{1} ;$ C.H. Shackleton ${ }^{2}$; G.P. Chrousos ${ }^{3} ;$ A. Hochberg ${ }^{1}$

${ }^{1}$ Rambam Medical Center, Haifa, Israel; ${ }^{2}$ Childrens Hospital,

Oakland, Calif.; ${ }^{3}$ NICHD/PREB, Bethesda, Md., USA

Following the removal of suprasellar tumors, some children develop severe $\mathrm{HyOb}$. Observation of Cushingoid habitus in these patients despite minimal glucocorticoid replacement, led us to characterize 11HSD activity and body composition. Eleven patients with $\mathrm{HyOb}$ and MPHD after removal of suprasellar tumors, aged 9-22 y, were studied. Five of these patients received GH replacement. Whole body DEXA and CT evaluation of visceral fat were compared to 6 patients with simple obesity $(\mathrm{SmOb})$. Glucocorticoid metabolites after a single oral dose of hydrocortisone acetate $\left(\mathrm{F}, 12 \mathrm{mg} / \mathrm{m}^{2}\right)$ or cortisone acetate $\left(\mathrm{E}, 15 \mathrm{mg} / \mathrm{m}^{2}\right)$ were compared to those of 5 patients with primary glucocorticoid deficiency (GD), receiving a similar dose. A complete 24-hour urine collection was analyzed by gas chromatography/mass spectrometry for 'A-ring' reduced $\mathrm{F}$ and $\mathrm{E}$ metabolites, i.e. tetrahydrocortisols (THF and allo-THF) and THE. In addition, urinary free cortisol (UFF) and free cortisone (UFE) were quantified using ${ }^{3} \mathrm{H}$-labeled internal standards. Total body fat (BF) was $47 \pm$ $8 \%$ and $57.2 \pm 2.47 \%$ (Mean \pm SD) in $\mathrm{HyOb}$ on and without GH therapy, resp. (n.s.), as compared to $49 \pm 7 \%$ in $\mathrm{SmOb}$ (n.s.). Lean body mass (LBM) was $53 \pm 7 \%$ and $39 \pm 6 \%$ in $\mathrm{HyOb}$ on and without $\mathrm{GH}$ therapy, resp (n.s), and $51 \pm 6 \%$ in $\mathrm{SmOb}$ (n.s.). Truncal /extremeties $\mathrm{BF}$ ratio was $0.9 \pm 0.09$ and $0.8 \pm 0.08$ in $\mathrm{HyOb}$ on and without $\mathrm{GH}$, resp (n.s.) and $1 \pm 0.07$ in $\mathrm{SmOb}(\mathrm{p}<$ 0.02 vs. $\mathrm{GH}$ treated $\mathrm{HyOb}$ ). Visceral fat was $74 \pm 58,44 \pm 25$ and $58 \pm 15$ $\mathrm{gr} / \mathrm{cm}^{2}$, resp. (n.s.). Visceral/subcutaneous fat ratio at the umbilical level was $0.2 \pm 0.05,0.19 \pm 0.02$ in $\mathrm{HyOb} \pm \mathrm{GH}$ (n.s.) and $0.14 \pm 0.03$ in $\mathrm{SmOb}(\mathrm{p}<$ $0.07, \mathrm{p}<0.06$, resp.). UFF/UFE ratio was calculated as a marker of renal $11 \mathrm{HSD} 2$ activity, and THF + allo THF/THE ratio as a marker of peripheral $11 \mathrm{HSD} 1$ activity. After oral loads of $\mathrm{F}$ or $\mathrm{E}$ :

\begin{tabular}{llllllll}
\hline & Load & $\mathrm{HyOb}-\mathrm{GH}$ & $\mathrm{HyOb}+\mathrm{GH}$ & $\mathrm{p}$ & $\mathrm{GD}$ & $\mathrm{p}-\mathrm{GH}$ & $\mathrm{p}+\mathrm{GH}$ \\
\hline UFF/UFE & $\mathrm{F}$ & $1.22 \pm 0.80$ & $0.85 \pm 0.74$ & 0.4 & $0.48 \pm 0.07$ & 0.03 & 0.4 \\
UFF/UFE & $\mathrm{E}$ & $1.23 \pm 0.67$ & $0.54 \pm 0.18$ & 0.09 & $0.78 \pm 0.6$ & 0.47 & 0.47 \\
THF/THE & $\mathrm{F}$ & $2.80 \pm 1.35$ & $2.35 \pm 1.27$ & 0.15 & $0.95 \pm 0.57$ & 0.01 & 0.11 \\
THF/THE & $\mathrm{E}$ & $1.92 \pm 1.00$ & $1.06 \pm 0.53$ & 0.18 & $1.76 \pm 0.6$ & 0.2 & 0.8 \\
\hline
\end{tabular}

Stratifying the results according to therapy, GH deficiency contributed $24 \%$ of the gained 11HSD1 activity. 11HSD1 activity correlated with visceral fat $(\mathrm{r}=$ $0.699, \mathrm{p}<0.01)$. Thus, $\mathrm{HyOb}$ is at least partially related to enhanced $11 \mathrm{HSD} 1$ activity, converting $\mathrm{E}$ to $\mathrm{F}$ in the liver and adipose tissue. GH deficiency partially accounts for this enhanced enzymatic activity and may be reversed by replacement therapy. Other hypothalamic controls are currently unknown. Lower dose and $\mathrm{E}$ rather than $\mathrm{F}$ replacement may minimize $\mathrm{HyOb}$

\section{P2-453 Fat Metabolism and Obesity}

Prevalence of Overweight and Obesity in North-Belgian Children and Adolescents

G. Massa ${ }^{1}$; E. Dezeure ${ }^{* 2}$;D. Lambert*2;P. Houben*2

${ }^{1}$ Virga Jesseziekenhuis, Hasselt, Belgium; ${ }^{2}$ Dept of School Health Care, Genk, Belgium

Aim: To assess the prevalence of overweight and obesity in children and adolescents of a North-Belgian area. Subjects and Methods: Height and weight were measured in 4704 children (age 3-14 years) during a regular visit at the centre for School Health Care between September 1998 and June 1999. The body mass index $\left(\mathrm{BMI}=\right.$ weight $/$ height $\left.^{2}\right)$ was calculated and compared to the Dutch references (Cole \& Roede, Ann Hum Biol, 1999), which are claimed to be relatively free of obesity. BMI-SDS was calculated by the LMS-method. Overweight was defined as BMI-SDS $>+1.3$ (percentile 90) and obesity as BMI-SDS $>+2$ (percentile 98). Results: Mean BMI SDS and percentage of children with overweight and obesity are reported in the table.

\begin{tabular}{|c|c|c|c|c|c|c|c|c|}
\hline \multirow{2}{*}{$\begin{array}{l}\text { Age } \\
\text { years }\end{array}$} & \multicolumn{4}{|c|}{ Boys } & \multicolumn{4}{|c|}{ Girls } \\
\hline & $\mathrm{n}$ & $\begin{array}{l}\text { BMI } \\
\text { SDS }\end{array}$ & $\begin{aligned} & \% \\
> & 1.3 \mathrm{SDS}\end{aligned}$ & $\begin{aligned} & \% \\
> & 2 \mathrm{SDS}\end{aligned}$ & $\mathrm{n}$ & $\begin{array}{l}\text { BMI } \\
\text { SDS }\end{array}$ & $\begin{aligned} & \% \\
> & 1.3 \mathrm{SDS}\end{aligned}$ & $\begin{aligned} & \% \\
> & 2 \mathrm{SDS}\end{aligned}$ \\
\hline $3-4$ & 505 & -0.06 & 10.5 & 4.0 & 446 & +0.01 & 10.1 & 4.3 \\
\hline $5-6$ & 472 & +0.10 & 15.5 & 7.4 & 454 & +0.21 & 16.3 & 6.8 \\
\hline $7-8$ & 486 & +0.30 & 18.1 & 7.8 & 478 & +0.31 & 20.3 & 9.6 \\
\hline $9-10$ & 340 & +0.44 & 26.2 & 14.1 & 301 & +0.40 & 24.9 & 12.6 \\
\hline $11-12$ & 467 & +0.56 & 31.0 & 18.8 & 386 & +0.47 & 29.8 & 11.1 \\
\hline $13-14$ & 208 & +0.59 & 31.3 & 17.3 & 161 & +0.59 & 29.8 & 14.9 \\
\hline
\end{tabular}

3-4-year-old children had a BMI distribution comparable to the obesity-free reference. The mean BMI starts to increase from the age of 5 years to reach a value of +0.59 SDS at adolescence. From the age of 5 years the prevalence of overweight increased progressively to reach a plateau of $30 \%$ at the age of 11 years. About one child in every 2 with overweight was obese resulting in an obesity prevalence of $17.3 \%$ and $14.9 \%$ in adolescent boys and girls, respectively. Conclusion: In line with data from other countries our findings show an increased overweight and obesity prevalence in children living in the North of Belgium. The prevalence starts to increase from the age of 5 years. Hence preventive measures have to begin at an early age, i.e. before the age of 5 years.

\section{P2-454 Fat Metabolism and Obesity \\ Prader-Willi Syndrome (PWS): Glucose Homeostasis and Insulin Secretion Remain Unchanged after 3 Years of Treatment with hGH as an Effect of Improved Body Composition \\ D. L'Allemand ${ }^{1}$; U. Eiholzer ${ }^{1}$; M. Schlumpf*1; T. Torresani ${ }^{2}$; J. Girard ${ }^{3}$ \\ ${ }^{1}$ Foundation Growth Puberty Adolescence, Zurich, Switzerland; \\ 2 University of Zurich, Zurich, Switzerland; ${ }^{3}$ Institute of Paediatric \\ Endocrinology, Basel, Switzerland}

In PWS, fasting insulin (FIns) levels (1) and beta-cell response (2) are reduced, in spite of obesity, being ascribed to a hypothalamic GH deficiency. However, since GH increases insulin (Ins) resistance, it has been questioned, if GH therapy (GHT) might precipitate the development of type 2 diabetes in PWS. Objective: We examined, if hyperinsulinism and related metabolic alterations develop on the long term under therapy with $3.4 \mathrm{U} \mathrm{hGH} / \mathrm{m}^{2} / \mathrm{d}$ during 36 months (m), measuring weight, fat mass (by DEXA), triglycerides (TG), HbA1c, FIns and glucose in 17 children (age 1.5-14.6 years) with PWS. Oral glucose tolerance and Ins secretion were tested (OGTT) in a subgroup of 13 and 12 children at 0 and $12 \mathrm{~m}$ of GHT, respectively. Results ( $\bar{x} \pm$ SEM): Before GHT, Ins secretion in OGTT (area under the curve, $\mathrm{AUC}_{\mathrm{Ins}}=536 \pm 123$ $\mathrm{pmol} / \mathrm{I}^{*} \mathrm{~h}$ ) was lower than shown in obese children, but markedly delayed $\left(\mathrm{t}_{\max }\right.$ $81 \pm 12 \mathrm{~min}$ ) compared to reference data (3), and did not significantly change under GHT $\left(661 \pm 68 \mathrm{pmol} / \mathrm{l}^{*} \mathrm{~h}\right)$. FIns, being normal (4) in 16 children before GHT $(46 \pm 14 \mathrm{pmol} / \mathrm{l})$, significantly increased at $12 \mathrm{~m}(72+12 \mathrm{pmol} / \mathrm{l})$ and

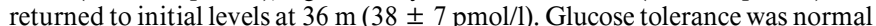
in 12 of 13 children before therapy, and in all under GHT; fasting glucose as well as HbAlc and TG were always normal. Fat mass before GHT was increased $(39 \pm 3 \%)$ and dropped into the upper normal range $(28 \pm 2 \%)$ under $36 \mathrm{~m}$ of GHT, being correlated with FIns $(\mathrm{r}=0.85, \mathrm{p}<0.001$, controlled for sex and age) at 0 and $12 \mathrm{~m}$. The increase of lean mass z-score, adjusted for age, sex and height, was significant only after $12 \mathrm{~m}$ of GHT (from $-1.4 \pm 0.2$ to $-0.85 \pm 0.3)$, but no more after $36 \mathrm{~m}(-1.1 \pm 0.4)$. Conclusions: In children with PWS, insulin secretion is low in relation to obesity and GH therapy. It is shown for the first time, that insulin secretion in OGTT is not elevated under $\mathrm{GH}$, and that the increase of fasting insulin is only transient; GHT during 3 years does not impair carbohydrate metabolism, but rather counteracts the potential $\mathrm{GH}$-induced insulin resistance by decreasing fat and increasing lean mass, which represents the metabolically active compartment.

1 Eiholzer U et al (1998). Eur J Pediatr 157:890-893.

2 Lindgren AC et al (1999). Horm Res 51:157-161.

3 Deschamps et al (1977). Diabetes 26:89-93.

4 Lautala P et al (1985). Acta Paediatr Scand 318(suppl):127-133. 
P2-455 Fat Metabolism and Obesity

The Lipodystrophic Syndrome in HIV-Infected Children D. Jaquet ${ }^{* 1} ;$ M. Levine ${ }^{* 2} ;$ E. Ortega*1; A. Faye*2; M. Polak ${ }^{1}$;

C. Lévy-Marchal*1

${ }^{1}$ INSERM U457, Paris, France; ${ }^{2}$ Robert Debré Hospital, Paris, France

The lipodystrophic syndrome described in HIV-infected adults encompasses different associations between abnormal fat redistribution, lipid and glucose metabolism disturbances. A cross-sectional study was implemented in HIVinfected children in order to investigate whether the lipodystrophic syndrome develops during childhood. 39 children, aged 3-18 years, underwent a clinical examination, fasting lipid measurement and an OGTT. Anthropometric data were expressed according to the French reference values for gender and age. Clinical lipodystrophy (LD) was defined as peripheral fat wasting (facial and/or buttock and/or limb atrophy with arm skinfold thicknesses below the 3rd percentile) and/or truncal adiposity (breast enlargement and/or buffalo neck and/ or relative abdominal obesity with trunk/arm skin fold ratio $2 \mathrm{SD}$ ). LD was observed in 13 children (33\%): 8 demonstrated truncal lipohypertrophy, 3 peripheral lipoatrophy and 2 combined lipodystrophy. Mean age did not significantly differ between the LD and non-LD groups $(9.6 \pm 3.8$ vs $8.9 \pm 4.3$ years, $\mathrm{p}=0.62$ ), but the combined LD were observed in adolescent only. Additionally, $\mathrm{LD}$ were more pronounced in the combined forms. Antiviral therapy, CD4 cell count and HIV viral load did not significantly differ between the LD and nonLD groups. The metabolic profiles of the two groups are given on the table.

\begin{tabular}{llll}
\hline Lipodystrophy & Present (13) & Absent (26) & $\mathrm{p}$ \\
\hline Triglycerides 95th percentile* $(\%)$ & 15 & 11 & 0.76 \\
Cholesterol 95th percentile* $(\%)$ & 23 & 15 & 0.59 \\
Fasting insulin $(\mu \mathrm{U} / \mathrm{ml})$ & $7.0 \pm 8.5$ & $3.0 \pm 2.3$ & 0.07 \\
Fasting insulin/glucose ratio & $1.58 \pm 1.9$ & $0.68 \pm 0.46$ & 0.07 \\
NGT $(\%) \neq$ & 100 & 100 &
\end{tabular}

* Reference values for gender and age Christensen et al.; $\$$ ADA criteria.

About $50 \%$ of the children demonstrated clinical and/or biological disturbances. Hyperinsulinemia occurs in LD children only, leading to frank insulinresistance in subjects with combined LD. Dyslipidemia were found in $23 \%$ of the non-LD children. Conclusion: The LD syndrome prevails in HIVinfected children in the three clinical forms initially described in adults but appears less pronounced before the initiation of puberty. Hyperinsulinemia associated with LD is also dramatically increased in the pubertal cases. We hypothesized therefore that puberty plays a critical role in the worsening of the whole syndrome. Finally, our data suggest that dyslipidemia observed in nonLD children reflect subclinical alterations of the adipose tissue.

\footnotetext{
P2-456 Fat Metabolism and Obesity
}

\author{
Responsiveness of Serum Leptin to Oral L-Dopa \\ Administration in GH-Deficient Children \\ T. Nakanishi*1; Y. Nakagawa*1;K. Toya ${ }^{1} ;$ A. Endoh ${ }^{* 1} ;$ K. Nasuda*1; \\ Y.-J. Liu*1;R.-S. Li*1;H. Saegusa*1;Y. Inaba*1 1; T. Ohzeki*1 \\ (Introduced by T. Torresani) \\ ${ }^{1}$ Hamamatsu, Japan
}

Recently, leptin was demonstrated to be controlled by factors other than fat cells. Objective: To evaluate whether serum leptin levels change in response to oral L-dopa administration. Patients and Methods: Serum leptin concentrations were measured using a radioimmunoassay kit (Linco Research Co.) in short children $(n=22)$ before and after L-dopa stimulation at growth hormone $(\mathrm{GH})$ provocative tests. The mean height and weight were $-2.7 \pm 0.7 \mathrm{SD}$ and $2.6 \pm 9.4 \%$ of the standard weight, respectively. Results: GH secretion was normal $(<10 \mathrm{ng} / \mathrm{ml})$ in 16 children and the peak values were below the limit in 6 patients. Serum leptin concentrations $(\mathrm{ng} / \mathrm{ml})$ were decreased after L-dopa (before $3.82 \pm 3.65$, after $3.47 \pm 4.84)$. In children with normal $\mathrm{GH}$ secretion, leptin levels were significantly $(\mathrm{p}=0.0137)$ lower after the stimulation $(2.36 \pm$
$0.76)$ than the basal values $(3.11 \pm 1.51)$. However, in GH-deficient patients, there was no significant difference $(p=0.5167)$ in leptin levels before and after oral L-dopa administration. Conclusions: Leptin was decreased in normal short children by direct or indirect stimulation with L-dopa.

\section{P2-457 Fat Metabolism and Obesity \\ Leptin, the Peripheral IGF-I System and Nutritional Parameters in Adolescent Female Athletes and Ballet Dancers}

M.T. Muñoz ${ }^{* 1}$; G. Garrido ${ }^{2}$; M. Buño*1; M. Chamorro*2; V. Barrios ${ }^{1}$; J.Pozo ${ }^{1} ; J^{\text {J.A }}$ rgente ${ }^{1}$

${ }^{1}$ Hospital Niño Jesús, Madrid, Spain; ${ }^{2}$ National Institute of Physical

Education, Madrid, Spain

In high competition athletes body fat stores tend to be greatly reduced. This could impede the normal development of adolescent athletes. Leptin and specific members of the GH-IGF-I system are thought to be good markers of nutritional status, as well as of the growth and reproduction status. Objectives: The objectives of this study were: 1) To determine serum leptin levels and their relationship to body mass in athletes. 2) To analyze possible alterations in the GH-IGF-I axis and their relationship to the nutritional status of these athletes. Patients and Methods: We have studied 38 females, 12 gymnasts aged 152 years (Group 1), 19 ballet dancers aged $15 \pm 2$ years (Group 2) and 7 triathelon athletes aged $16 \pm 1$ years (Group 3). The control group consisted of 37 healthy adolescents of the same sex and age. All athletes participated in physical exercise for more than 18 hours/week. Nutritional intake was determined via a questionnaire analyzing 5 days with the Nutrionist IV ${ }^{\circledR}$ program. The body mass index (BMI) was calculated and expressed as standard deviation for the age and sex. Serum IGF-I, IGFBP-3, GHBP and leptin levels were determined by RIA and IGFBP-1 by ELISA. The presence of menarche and regular menstrual cycles was also analyzed. Results: Serum IGF-I, IGFBP-I and 3 levels were normal in all three groups. GHBP levels were significantly decreased in those athletes that presented primary ammenorhea or irregular menstrual cycles $(\mathrm{p}<0.01)$. There was also a significant correlation between BMI and GHBP concentrations $(\mathrm{p}<0.05)$. Leptin level were significantly reduced in all athletes compared to controls and there was a significant correlation between leptin and BMI ( $\mathrm{r} 0.59, \mathrm{p}<0.01)$. The nutritional intake in groups 1 and 2 was adequate for their age and sex, but probably inadequate for the levels of physical exercise. Conclusions: 1) The gymnasts and ballet dancers present alterations in parameters of the peripheral GH-IGF axis which could be related to their nutritional state, and even more to the hypoestrogenism. 2) Leptin is a good biochemical marker of the nutritional status in these athletes, being reduced in parallel with the reduction in total body fat. 
P2-458 Fat Metabolism and Obesity

\section{Whole Body Air Displacement Plethysmography for Body Composition Analysis in Obese Children and Adolescents}

C. Azcona ${ }^{1}$; S. Raggio*11; M. Ramos*1; E. Ruza*1; G. Frühbeck*1 ${ }^{1}$ Pamplona, Spain

Introduction: Obesity is the most frequent type of malnutrition among children and adolescents from developed countries. Body composition analysis is of great value in this group of patients in order to make an accurate diagnose and guide medical intervention. The proportions of fat mass (FM) and fat free mass (FFM) can be derived from body density (mass/volume) using Archimedes' principle, assuming constant densities for measuring body density. This approach involves full submersion of the subject under water. The recent development of whole body air displacement plethysmography is minimally invasive and does not require highly trained investigators. Objective: To assess body composition in obese children and adolescents using whole body air displacement plethysmography. Material and Methods: Twelve patients ( 7 males and 5 females) were included in our study. Obesity was defined with regard to body mass index (BMI) (2SDS). Whole body air displacement plethysmography was performed using BOD-POD body composition system. Resting energy expenditure (REE) was assessed by indirect calorimetry. Serum levels of cholesterol, triglycerids, glucose, insulin, leptin and lipoprotein 'a' were also measured. Results:

\begin{tabular}{lllll}
\hline & Mean & CI: 95\% & CI: $5 \%$ & Normal \\
\hline Age & 11.6 & 10.2 & 13.1 & \\
Weight (SDS) & 2.3 & 1.8 & 2.8 & \\
BMI (SDS) & 2.7 & 2.4 & 3.0 & \\
FM\% & 41.2 & 37.0 & 45.4 & $<20 \%$ \\
FFM \% & 58.9 & 54.7 & 63 & \\
REE\% & 111.2 & 102.6 & 119.8 & \\
REE (kcal/day) & 1794.8 & 1638.6 & 1950.8 & \\
Cholesterol (mg/dl) & 181.7 & 163.9 & 199.5 & $<2.2$ \\
LDL/HDL & 2.3 & 1.7 & 2.8 & \\
Triglycerids (mg/dl) & 90.9 & 65.5 & 116.4 & \\
Glucose (mg/dl) & 93.2 & 89.9 & 96.5 & $5-25$ \\
Insulin (UI/l) & 20.9 & 16.1 & 95.8 & \\
Leptin ( $\mu$ g/l) & 29.9 & 18.7 & 41.2 & 0 \\
Lipoprotein 'a' (mg/dl) & 42.6 & 24.4 & 60.8 & $0-30$ \\
\hline
\end{tabular}

Weight $(\mathrm{kg}), \mathrm{BMI}$ and leptin are significantly correlated with $\mathrm{FM} \%(\mathrm{r}=0.80$; $\mathrm{p}<0.05)$. Weight $(\mathrm{kg})$ and insulin are correlated with REE $(\mathrm{kcal} /$ day $)(\mathrm{r}=0.72$; $\mathrm{p}<0.01$ and $\mathrm{r}=0.56 ; \mathrm{p}<0.05$, respectively).

Conclusion: FM percentage determined by whole body air displacement plethysmography was performed using BOD-POD body composition system BOD-POD is increased in children with obesity. REE in obese children and adolescents is is increased in children with obesity. REE in obese children and adolescents is
elevated. BOD-POD system is an easy and non-invasive method to assess body composition in children and adolescents with obesity.

P3-459 Fat Metabolism and Obesity

Serum Leptin in Formerly Small for Gestational Age (SGA) Children: Relationship to Gender, Puberty, Body Composition, Insulin Sensitivity, and Serum Uric Acid F. Pulzer*1; J. Kratzsch ${ }^{* 1}$; V. Richter ${ }^{* 1} ;$ F. Rassoul*1; W. Kiess ${ }^{1}$; E. Keller ${ }^{1}$;

${ }^{1}$ University of Leipzig, Leipzig, Germany

Objective: Serum leptin levels have been described to be associated with serum uric acid in humans. Furthermore, former SGA subjects are reported to have an impaired renal function and are known to have a higher risk of developing complications related to syndrome $\mathrm{X}$. We therefore aimed to evaluate the interrelationship between leptin and markers of the metabolic syndrome in former SGA children. Methods: The relation between serum leptin concentration, body composition, lipid parameters, calculated insulin resistance (HOMA), and uric acid (UA) levels was studied in 50 former SGA children: 17 boys (mean age $15.5 \pm 2.9$ years), and 33 girls (mean age $15.0 \pm 3.4$ years). Each subject underwent an oral glucose tolerance test. Results: We found a strong positive association between leptin and estimates of body fatness, and female gender. Eleven children with impaired glu- cose tolerance (IGT) had higher UA levels than subjects with normal glucose tolerance $(4.9 \pm 1.0$ vs. $4.2 \pm 1.1 \mathrm{mg} / \mathrm{dl}, \mathrm{p}<0.05)$, and showed the strongest relation between serum leptin and UA $(r=0.78, p<0.001)$. Multiple regression analysis demonstrated that body fat mass (partial $\mathrm{r}=0.88, \mathrm{p}<0.01$ ), gender (partial $\mathrm{r}=$ $0.83, \mathrm{p}<0.01)$, birth weight (partial $\mathrm{r}=-0.81, \mathrm{p}<0.01)$, fasting glucose $($ partial $\mathrm{r}=$ $0.68, \mathrm{p}<0.05)$, and uric acid (partial $\mathrm{r}=0.70, \mathrm{p}<0.05$ ) were independently associated with serum leptin $\left(\mathrm{R}^{2}=0.89, \mathrm{p}<0.001\right)$ in former SGA children with IGT. Conclusions: Serum leptin levels tend to be higher in children with IGT and do correlate positively with serum uric acid levels, and indices of body fatness, and inversely with birth weight. This suggests that renal leptin degradation, and uric acid excretion might be impaired already in formerly intrauterine retarded subjects with dysglycemia at younger age.

\section{P3-460 Fat Metabolism and Obesity \\ Obesity in Septo-Optic Dysplasia (SOD), and Response to GH \\ J. Kirk ${ }^{1} ;$ K. Waldron* \\ ${ }^{1}$ Birmingham Children's Hospital, Birmingham, United Kingdom}

Introduction: Septo-optic dysplasia (SOD) consists of a triad of optic nerve hypoplasia $(\mathrm{ONH})$, hypopituitarism and midline defects (including absent septum pellucidum). Although most present with failure to thrive, within our clinic we have been increasingly recognising patients who are significantly obese. Patients: Eight patients have now been recognised ( 5 male). All have ONH and hypopituitarism (anterior in 3, posterior in 1, combined in 4), with appropriate replacement with hydrocortisone, thyroxine and DDAVP. Six had anterior pituitary function, $\&$ all are $\mathrm{GH}$-insufficient (peak $\mathrm{GH}<15 \mathrm{mU} / 1$ ). In all 8 patients serum IGF-1 has been within the normal range for age. Auxology: Birthweight was normal: 10-50th centile in 7,90th centile in 1 . At the most recent assessment (or pre-GH therapy) patients were a mean of 6.1 years (range 2.5-12.6). Mean BMI SDS was +3.45 (range +1.71 to +6.5 ). Despite evidence of hypopituitarism only 3 patients were short (height SDS $<-1.8$ ), and 4 slowly growing (HV $<-0.8$ SDS). GH Therapy: 3 patients ( 2 slowly growing) have been treated with replacement doses of $\mathrm{GH}$. During the first year of therapy all showed an increase in $\mathrm{HV}<2 \mathrm{~cm} / \mathrm{y}$, but in addition an increase in BMI SDS. Conclusions: These data indicate that a number of patients with SOD are obese, and continue to grow normally and maintain a normal IGF-1 despite evidence of GH-insufficiency; so called 'eating themselves out of trouble'. Administration of GH may improve growth rate, but has no effect on obesity.

\section{P3-461 Fat Metabolism and Obesity}

Sex Hormone Binding Globulin (SHBG) Levels Are Low in Obese Prepubertal Children with Hyperinsulinaemia

P. Galloway*1; A.M. Wallace*2 ${ }^{*}$ M. Donaldson ${ }^{1}$ (Introduced by $\overline{M . D . C . ~ D o n a l} d s o n)$

${ }^{1}$ Royal Hospital for Sick Children, and ${ }^{2}$ Institute of Biochemistry,

Glasgow Royal Infirmary, Glasgow, United Kingdom

Insulin resistance (IR) is implicated in the pathogenesis of polycystic ovary syndrome, type 2 diabetes mellitus and ischaemic heart disease. Childhood obesity, which is becoming increasingly common, may be associated with IR. A screening test for IR in obese children would help target those most in need of intervention. SHBG, a stable glycoprotein under control of insulin, androgens and thyroxine, is a possible 'marker' of IR. In a previous study, normative SHBG concentrations were established in 57 pre-pubertal children (30F:27M; age 5-10 years) of 35-168 nmol/ 1 in males and $44-143 \mathrm{nmol} / 1$ in females. In a further 71 children of pubertal age (11-17 years) SHBG levels decreased, coincident with a period of relative insulin resistance. Objective: To examine the relationship between SHBG and hyperinsulinaemia in obese prepubertal children. Patients and Methods: SHBG was measured by immunoradiometric assay in 32 healthy prepubertal children, comprising 25 obese children (15F:10M; age 3.2-13 years; BMI 2SD for age) and 7 controls (4F:3M; age $6.1-10.1$ years) following an oral glucose tolerance test. Hyperinsulinaemia was defined as area under insulin/time curve $100 \mathrm{pmol} / 30$ min. Results: The non-obese controls had normal SHBG levels and normal insulin responses to oral glucose load. Of the 25 obese children, 14 (10F:4M) had hyperinsulinaemia with SHBG $35 \mathrm{nmol} / 1$ in all but two (46 and $50 \mathrm{nmol} / \mathrm{l})$. SHBG levels in the 11 children $(5 \mathrm{~F}: 6 \mathrm{M})$ with normal insulin responses were normal in all but two girls (19 and $36 \mathrm{nmol} / 1)$. Conclusion: SHGB measurement in this group of prepubertal obese children had a specificity of $89 \%$ and a sensitivity of $86 \%$ in predicting hyperinsulinaemia. 


\section{P3-462 Fat Metabolism and Obesity}

\section{Leptin Serum Concentration Increases after Major} Surgery

J. Dötsch*1; R. Wagner ${ }^{1} ;$ M. Grösch ${ }^{1}$; J. Scharf1; H. Singer ${ }^{1}$;

N. Katz' ; H.G. Dörr ${ }^{1}$;W. Rascher ${ }^{1}$;

${ }^{1}$ University of Erlangen-Nürnberg, Erlangen, Germany

Besides a central action, the peripheral regulation of leptin has become a new focus of research. Glucocorticoids and catecholamines, both involved in blood pressure control, have been shown to regulate leptin synthesis. The objective of the present study was to examine the impact of major heart surgery with cardiopulmonary bypass $(\mathrm{CPB})$ in childhood on leptin serum concentrations in relation to glucocorticoids and adrenaline. Leptin, cortisol and adrenaline concentrations of twenty patients between 1 month and 15 years who underwent open heart surgery were compared to 18 children between 1 month and 17 years with major surgery not necessitating CPB (control group). Leptin and cortisol were measured by radioimmunoassay, adrenaline was determined by HPLC. Leptin serum concentrations gradually increased from $0.4 \pm 0.1 \mathrm{ng} / \mathrm{ml}$ (mean \pm SEM) preoperatively to $1.5 \pm$ $0.7 \mathrm{ng} / \mathrm{ml} 12$ hours after surgery $(\mathrm{p}<0.001)$ and declined thereafter in the group with CPB. Showing similar kinetics, leptin slowly rose from $0.5 \pm 0.2 \mathrm{ng} / \mathrm{ml}$ before surgery to $2.2 \pm 1.1 \mathrm{ng} / \mathrm{ml}$ after 12 hours in the control group $(\mathrm{p}=0.001)$. Preoperatively, plasma cortisol was $48 \pm 11 \mu \mathrm{g} / \mathrm{dl}$ in the CPB group, where all patients received prednisone during surgery, and consequently gradually decreased to $11 \pm$ $2 \mu \mathrm{g} / \mathrm{dl} 12$ hours after surgery $(\mathrm{p}=0.06)$. In the control group cortisol concentrations were $28 \pm 10 \mu \mathrm{g} / \mathrm{dl}$ before and $13 \pm 3 \mu \mathrm{g} / \mathrm{dl} 12$ hours after surgery $(\mathrm{p}=0.20)$. Adrenaline increased from $26 \pm 3 \mathrm{pg} / \mathrm{ml}$ before to $289 \pm 66 \mathrm{pg} / \mathrm{ml} 12$ hours after surgery $(\mathrm{p}<0.001)$ in the CPB group. In the control group adrenaline increased from $21 \pm 5$ to $86 \pm 24 \mathrm{pg} / \mathrm{ml}(\mathrm{p}=0.03)$. There is a significant increase in leptin serum concentration after major surgery with and without CPB. Patients with CPB showed a similar increase in serum leptin concentrations than the controls. Neither was adrenaline able to suppress postoperative leptin secretion nor could glucocorticoids stimulate leptin secretion. Therefore, a more powerful stimulus of leptin secretion after major surgery or anaesthesia has to be postulated. These results may be discussed with regard to the role of leptin in raising blood pressure.

\section{P3-463 Fat Metabolism and Obesity}

\section{Fasting GH Increases after a 7 Month Weight Loss in} Obese Adolescents, Independently of Free IGF I

J.C. Souberbielle ${ }^{* 1} ; H$. Thibault ${ }^{* 2} ;$ F. Chevenne ${ }^{* 1} ;$ L. Bussieres ${ }^{* 1}$; D. Roinsol ${ }^{* 3} ;$ D. Soulie ${ }^{* 3} ;$ M.F. Cachera ${ }^{2} ;$ P. Serog ${ }^{* 3}$ (Introduced by Raja Brauner)

${ }^{1}$ Hôpital Necker Enfants Malades, Paris, France; ${ }^{2}$ ISTNA - CNAM,

Paris, France; ${ }^{3}$ 'Les Oiseaux', Sanary/mer, France

In obese subjects, a blunted GH secretion which recovers during weight loss is a well-known feature. An increase in the free fraction of IGF I due to reduced IGFBP1 as a consequence of hyperinsulinism has been said to be partly responsible IGFBP1 as a consequence of hyperinsulinism has been said to be partly responsible
for a negative feed-back on GH secretion. We aimed to clarify this hypothesis by measuring fasting serum GH (by means of a new ultrasensitive immunoassay with a detection limit of $5 \mathrm{pg} / \mathrm{ml}$ ), total and free IGF I, IGF-BP1, 2 and 3, leptin and insulin in 90 obese adolescents ( 24 boys, 66 girls; aged $14.3 \pm 1.3$ years at inclusion) before and after 2 weeks and $7.1 \pm 1.1$ months of a weight-reducing program which consisted in a low-energy diet $(1750 \mathrm{kcal} / \mathrm{d})$ and a high level of exercise. Results (mean \pm sem) are summarized in the following table:

\begin{tabular}{lccc}
\hline & Before & 2 Weeks & 7 Months \\
\hline Weight $(\mathrm{kg})$ & $97.1 \pm 1.4$ & $91.2 \pm 1.9^{*}$ & $69.8 \pm 1.0^{*}$ \\
BMI $\left(\mathrm{kg} / \mathrm{m}^{2}\right)$ & $36.0 \pm 0.5$ & $34.4 \pm 0.5^{*}$ & $25.1 \pm 0.3^{*}$ \\
Serum GH $(\mathrm{pg} / \mathrm{ml})$ & $868 \pm 184$ & $633 \pm 151$ & $1842 \pm 321^{*}$ \\
Serum tot. IGF I (ng/ml) & $442 \pm 16$ & $441 \pm 17$ & $445 \pm 17$ \\
Serum free IGF I (ng/ml) & $3.1 \pm 0.2$ & $2.8 \pm 0.1$ & $3.0 \pm 0.1$ \\
Serum IGFBP1 (ng/ml) & $17.2 \pm 1.3$ & $18.0 \pm 1.5$ & $35.8 \pm 2.1^{*}$ \\
Serum IGFBP2 (ng/ml) & $256 \pm 21$ & $287 \pm 23$ & $391 \pm 18$ \\
IGFBP3 (mg/l) & $4.2 \pm 0.1$ & $4.1 \pm 0.1$ & $4 . \pm 0.1$ \\
Serum insulin (mU/l) & $23.6 \pm 1.1$ & $18.1 \pm 0.6^{*}$ & $9.3 \pm 0.4^{*}$ \\
Serum leptin (ng/ml) & $37 \pm 2$ & $23 \pm 1^{*}$ & $11 \pm 1^{*}$ \\
\hline
\end{tabular}

* $\mathrm{p}<0.05$ or less vs. 'before' (Wilcoxon test).
Fasting serum GH was not correlated with total or free IGF I at any timepoint. Similarly, we found no correlation between change in serum GH and change in other biological parameters or weight or BMI at $5 \mathrm{mo}$. Taken together, these preliminary results cannot demonstrate that increase in fasting serum $\mathrm{GH}$ during weight loss in obese adolescent is primarily dependent on free IGF I. The exact physiological meaning of fasting serum $\mathrm{GH}$ as well as the real specificity of the free IGF I assay used in our study deserve further evaluation.

P3-464 Fat Metabolism and Obesity

\section{Circulating Monocyte and T-Lymphocyte-Specific} Chemokines Are Elevated in Overweight Adolescents

I. Magaziotou*1;D. Domianaki*1; I. Elefsiniotis*2; E. Economou*2

A. Mariolis*2; C. Stefanadis*2;P. Toutouzas ${ }^{* 2}$ (Introduced by H. Theodoridis)

1 Tzanio Hospital, Athens, Greece; ${ }^{2}$ University of Athens,

Hippokration Hospital, Athens, Greece

The long-term risks that arise from overweight, in children and adolescents, are associated, among others, with atherosclerosis. Representing the very early step of endothelial activation, monocyte- and T-lymphocyte-specific chemotaxis mostly and eosinophil-specific chemotaxis much less, all involved in inflammatory response, are of fundamental importance in the development of atherosclerosis. Monocyte chemoattractant protein-1 (MCP-1), RANTES and EOTAXIN (EOX) are monocyte, T-lymphocyte and eosinophil-specific chemokines involved in the directed locomotion ta and trasmigration across to the endothelium at the sites of inflammation. Objective: To evaluate the potential role of circulating forms of MCP-1, RANTES and EOX as a risk factor for cardiovascular disease in overweight adolescents. Patients and Methods: Twenty-four (24) overweight adolescents (10-14 years, 13 females and 11 males) with body mass index (BMI) greater than the 95th percentile on the growth charts from the National Center of Health Statistics, were evaluated for serum levels of MCP-1, RANTES and EOX and compared to fourteen (7 females and 7 males) age-, sex- and BMI-matched lean adolescents with BMI less than the 75th percentile. Serum levels of MCP-1 RANTES and EOX were measured by Blisa. Adolescents with genetic syndromes or endocrine causes of obesity, inflammatory or systemic diseases were excluded from the study. Results: The results can be summarized as follows:

\begin{tabular}{llll}
\hline Study group & MCP-1 $(\mathrm{pg} / \mathrm{ml})$ & RANTES $(\mathrm{pg} / \mathrm{ml})$ & EOX $(\mathrm{pg} / \mathrm{ml})$ \\
\hline Overweight & $240 \pm 14.9^{\mathrm{a}}$ & $562 \pm 54.4^{\mathrm{a}}$ & $82.2 \pm 5.4^{\mathrm{a}}$ \\
Lean & $207 \pm 22.7$ & $543 \pm 72.9$ & $104 \pm 5.0$
\end{tabular}

Significantly different (Mann-Whitney $U, p<0.05$ compared to those of lean group).

The significant correlations between MCP-1/EOX, MCP-1/RANTES and EOX/ RANTES serum levels noticed in lean group $(\mathrm{r}=0.6, \mathrm{p}=0.0001, \mathrm{r}=0.54, \mathrm{p}=$ $0.02, r=0.6, p=0.009)$ were found to be still active in overweight group $(r=$ $0.39, \mathrm{p}=0.021, \mathrm{r}=0.64, \mathrm{p}=0.0001, \mathrm{r}=0.48, \mathrm{p}=0.01)$ emerging the concept that the complex interactions among monocytes, T-lymphocytes and eosinophils possibly forming a functional unit, are still active. Conclusions: The results suggest that, although cross talk among monocytes, T- lymphocytes and eosinophils seems to be unaltered, the most important types of leukocyte chemotaxis for the development of atherosclerosis, namely monocyte- and T-lymphocyte-specific chemotaxis are both elevated in overweight adolescents. This may indicate that the very early step of endothelial inflammation has already started. 
P3-465 Fat Metabolism and Obesity

\section{Plasma Levels of Total Homocysteine Are Elevated in} Overweight Adolescents

J. Magaziotou $^{* 1} ;$ D. Damianaki*1;E. Economou*2; J. Elefsiniotis ${ }^{* 2}$; A. Mariolis*2; C. Stefanadis*2;P. Toutouzas*2 (Introduced by

H. Theodoridis)

${ }^{1}$ Tzanio Hospital, Piraeus, Greece; ${ }^{2}$ University of Athens,

Hippokration Hospital, Athens, Greece

Childhood and adolescent obesity is proposed to predict atherosclerosis and cardiovascular disease. At the same time mild to moderate elevation of plasma total homocysteine is regarded as and independent risk factor for cardiovascular disease. Objective: To evaluate levels of total-homocysteine (t-HCY) in plasma of overweight adolescents. Patients and Methods: Twenty-four (24) overweight adolescents (10-14 years, 13 females and 11 males) with body mass index (BMI) greater than the 95th percentile on the growth charts from the National Center of Health Statistics, were evaluated for EDTA-plasma levies of t-HCY and compared to fourteen ( 7 females and 7 males) age-, sex- and BMImatched lean adolescents with BMI less than the 75th percentile. The EDTAplasma levels of t-HCY were measured on the Abbott IMx analyzer using fluorescence polarization immunoassay technology. Adolescents with genetic syndromes or endocrine causes of obesity, hyperlipidemia and deficiency of enzymes involved in the metabolization of homocysteine were excluded from the study. Results: Plasma levels of t-HCY were found to be significantly higher in overweight than in lean adolescents $(8.4 \pm 0.5 \mu \mathrm{mol} / 1 \mathrm{vs} 7 \pm 0.3 \mu \mathrm{mol} / \mathrm{l}$, $\mathrm{p}=0.02$ ). No significant differences in plasma levels of $\mathrm{t}-\mathrm{HCY}$ were found between females and males in both overweight $(8.3 \pm 0.5$ and $8.4 \pm 0.9 \mu \mathrm{mol} / \mathrm{l}$, $\mathrm{p}=0.9)$ and lean $(6.9 \pm 0.5$ and $7 \pm 0.5 \mu \mathrm{mol} / \mathrm{l}, \mathrm{p}=0.9)$ groups. Although significant relation was noticed in lean group between plasma levels of t-HCY and BMI $(r=0.54, p=0.02)$, this was not noticed in over- weight group. Conclusions: An unrelated to BMI elevation of plasma t-HCY is noticed in overweight adolescents and this may confer an independent risk for cardiovascular disease.

P3-466 Fat Metabolism and Obesity

\section{Tumor Necrosis Factor (TNF- $\alpha$ ) and Leptin Levels in} Childhood Obesity

E. Vlachopapadopoulou ${ }^{1} ;$ F. Karachaliou $^{1} ;$ A. Fotinou ${ }^{* 2}$. E. Paraskaki*2;E. Konstadellos*3; S. Michalacos*1

Depts of ${ }^{1} \mathrm{Growth}$ and Development and ${ }^{2}$ Microbiology, Children's Hospital P \& A Kyriakou, Athens, Greece; ${ }^{3}$ General Hospital of

Nikaia, Athens, Greece

TNF- $\alpha$ is a cytokine with multiple actions. It has been shown that TNF- $\alpha$ mRNA expression is increased in fat cells in obese rodents as well as in humans. On the contrary activation of the TNF- $\alpha$ system has been associated with weight loss in humans. Objective: To investigate whether circulating levels of TNF- $\alpha$ are related to serum leptin levels, percentage of body fat and insulin levels in obese prepubertal children. Patients and Methods: The study population consisted of 33 prepubertal children (14 boys) aged $9.2 \pm 1.9$ years with exogenous obesity (BMI $>2$ SDs for age and sex) and 23 prepubertal children ( 9 boys) of normal weight who served as controls. After an overnight fast, insulin, leptin and TNF- $\alpha$ levels were drawn and body composition was assessed using bioelectrical impedance (STA/BIA, Akern). Leptin was measured in duplicate by radioimmunoassay (Linco, MA) and TNF- $\alpha$ with a microelisa kit (R-D systems). Statistical analysis was performed using non-parametric methods (SPSS 8.0). Results: (All values are expressed as mean \pm SEM). BMI was $26.09 \pm$ 0.3 and $\mathrm{BMI}_{\text {SDS }} 2.8 \pm 0.08$. Insulin levels were $1.5 \pm 1.4 \mu \mathrm{IU} / \mathrm{ml}$. Leptin levels were $22.8 \pm 2.2 \mathrm{ng} / \mathrm{ml}$ and TNF- $\alpha$ was $9.9 \pm 1.9 \mathrm{pg} / \mathrm{ml}$. Fat percentage was $36.1 \pm 3.8 \%$. Leptin levels were significantly higher in the obese children than in the lean $(\mathrm{p}<0.001)$. However, TNF- $\alpha$ levels did not differ between the obesity and the control group. It is of interest though that 8 obese children had elevated values of TNF- $\alpha$ with no apparent reason. TNF- $\alpha$ did not correlate with BMI, fat percentage insulin or leptin levels. Leptin levels correlated with $\operatorname{BMI}(r=0.4, p=0.002)$ and with fat percentage $(r=0.7, p<0.001)$. Conclusions: Our data suggest that there is no correlation of TNF- $\alpha$ levels with insulin or leptin levels and with BMI, or body fat percentage in obese prepubertal children.

39th Annual Meeting of the ESPE
P3-467 Fat Metabolism and Obesity

Study of Insulin Resistance Parameters in Childhood Obesity

O. Noizet*1; J. Weill1; M. Cartigny*1; C. Stuckens*1

Pediatric Endocrine Unit, University Hospital, Lille, France

Introduction: Obesity induces insulin resistance (IR) and hyperinsulinism (HI). Aims of the Study: To characterize the frequency of IR in childhood obesity and to correlate insulinemia with anthropometric data and with other assumed IR criteria: IGF BP-1 and triglycerid plasma levels during an oral glucose tolerance test (OGTT: 1.75 g glucose/kg BW). Patients/Methods: 36 frankly obese children without glucose intolerance during OGGT; 19 girls, 16 boys; age 3 to 15.5 years $(5.2+3.28) ; 12$ pubertal, 23 prepubertal or early pubertal; weight/height 0.11 to 11 $\mathrm{SD}(6.1 \pm 2.52)$. Results:

\begin{tabular}{lllll}
\hline & Median & SD & $\begin{array}{l}\text { 5th } \\
\text { percentile }\end{array}$ & $\begin{array}{l}\text { 95the } \\
\text { percentile }\end{array}$ \\
\hline Fasting insulinemia (mUI/l) & 8.15 & 8.87 & 0 & 28.8 \\
Insulinemia OGTT peak (mUI/l) & 71.8 & 72.45 & 28 & 197 \\
Fasting IGF BP-1 (mg/l) & 3.8 & 6.38 & 0 & 19 \\
Minimum OGTT IGF BP-1 (mg/l) & 0 & 1.97 & 0 & 4.1 \\
Fasting triglyceridemia (mg/l) & 0.78 & 0.45 & 0.37 & 1.76 \\
Triglyceridemia CGTT peak (mg/l) & 1.01 & 0.52 & 0.43 & 2.29 \\
\hline
\end{tabular}

Another worthy correlation $(\mathrm{R}=-0.99, \mathrm{p}=0.0001)$ was found between log basal insulinemia and quotient log basal insulinemia/glycemia, which validates insulinemia as an approach of IR. No correlation was found between basal insulin level and insulin peak level, anthropometric data, IGF BP-1, triglycerids, nor in basal state neither during OGTT. However, when glycemia was superior to $0.90 \mathrm{~g} / 1, \mathrm{HI}$ was constant and some patients with low insulin levels were also characterized by low IGF BP-1 levels. Conclusions: Insulin resistance is independant from amplitude and time obesity data. A still normal, more than $0.90 \mathrm{~g} / \mathrm{l}$ fasting glycemia, is indicative of insulin resistance and suggests a risk for later non insulin-dependent diabetes. A normal basal insulinemia does not exempt from performing OGTT. Some patients with low insulinemia and IGF BP-1 levels should be obese because hypersensitive to insulin.

\section{P3-468 Fat Metabolism and Obesity \\ Relations of Cortisol with Insulin Resistance in Childhood Obesity}

O. Noizet*1; J. Weill ${ }^{1}$; M. Cartigny*1; C. Stuckens ${ }^{* 1}$

${ }^{1}$ Pediatric Endocrine Unit, University Hospital, Lille, France

Introduction: Cortisol status is not clear in obesity, especially in childhood. Aims of the Study: To characterize the status of cortisol and its binding protein, CBG, in obese children and to assess during an oral glucose test tolerance (OGTT) its relation with insulin resistance, an important hallmark of obesity. Patients: 35 frankly obese children without glucose intolerance; 19 girls and 16 boys; age 3 to 158.5 years $(5.2 \pm 3.28) ; 12$ pubertal and 23 prepubertal or early pubertal; weight/height 0.11 to $11 \mathrm{SD}(6.1 \pm 2.52)$. Results:

\begin{tabular}{lllllc}
\hline & Median & SD & $\begin{array}{l}\text { 5th } \\
\text { percentile }\end{array}$ & $\begin{array}{l}\text { 95th } \\
\text { percentile }\end{array}$ & $\begin{array}{l}\text { Normal } \\
\text { values }\end{array}$ \\
\hline 0.8 a.m. cortisolemia (g/l) & 130 & 41.6 & 60 & 194 & $90-220$ \\
0.4 p.m. cortisolemia (g/l) & 50 & 26.9 & 18 & 91 & $40-120$ \\
24 h free urinal cortisol $(\mu \mathrm{g} / \mathrm{D})$ & 45 & 19.8 & 19 & 81 & $20-110$ \\
Fasting CBG (nmol/l) & 987 & 114 & 670 & 1053 & $675-900$ \\
Fasting insulin (mUI/l) & 8.15 & 8.87 & 0 & 28.8 & inf to 20 \\
Insulinemia OGTT peak (mUI/l) & 71.8 & 72.4 & 28 & 197 & inf to 133 \\
\hline
\end{tabular}

Correlations: There was no correlation between insulin resistance parameters (basal and peak insulinemia, Insulin Growth Factor Binding Protein-1, triglycerids) and cortisol parameters, excepted 04 p.m. cortisolemia $(\mathrm{p}=0.016)$. Conclusions: Contrarily to previous assertions, cortisol is not high but rather low in childhood obesity. These low levels are not due to the decrease of its main binding protein, CBG. A higher 04 p.m. cortisol level is related to insulin resistance, although not affecting global free cortisol urinary excretion. 
P3-469 Fat Metabolism and Obesity

\section{Phenotypic Characterization and Analysis of MC4-R Gene in $\mathbf{3 0}$ Children Presenting with Precocious Obesity}

B. Jouret ${ }^{1}$; M. Gayrard ${ }^{1} ;$ F. Marin $^{1}$; P. Frogue ${ }^{2}$; M. Tauber ${ }^{1}$

${ }^{1}$ Hôpital des Enfants, Toulouse, France; ${ }^{2}$ Institute Pasteur de Lille, Lille, France

We have precisely analyzed the clinical, biological phenotype and the melanocortin 4 receptor gene (MC4-R) - a key element in the control of food consumption - in 30 children ( 23 girls +7 boys) presenting with severe and precocious obesity (before 6 years). Mean BMI at inclusion was $22.8 \mathrm{~kg} / \mathrm{m}^{2}(6.40$ SDS for BMI), mean waist circumference was $70 \mathrm{~cm}$ and mean waist/hip ratio was 0.94 mean father's BMI was $27.2 \mathrm{~kg} / \mathrm{m}^{2}$ and mean mother's BMI $25.6 \mathrm{~kg} / \mathrm{m}^{2} .35 \%$ of the children had one obese parent and $40 \%$ two obese parents. We found a positive correlation between the BMI of the mother but not the father and the BMI of the child at inclusion and from 3 years old $(p=0.037$ and $p<0.0001)$. BMI at inclusion positively correlated with the waist circumference $(p<$ $0.0001)$, lean body mass $(\mathrm{p}=0.006)$, fat mass $(\mathrm{p}=0.007)$ measured by dual absorptiometry. Mean fasting insulin was $6 \mathrm{IU} / 1$ and mean insulin peak on OGTT was very elevated at $73 \mathrm{IU} / 1$. Mean plasma POMC value was elevated at $141 \mathrm{IU} / \mathrm{ml}$ while ACTH and free urinary cortisol were normal. The single exon of the MC4-R gene was analyzed by denaturant gel electrophoresis (DGGE) after PCR amplification for the 30 patients. Two abnormal migration profiles were found. The first one corresponded to a known polymorphism V103I (found in $6.9 \%$ of obese adults vs $4.3 \%$ lean adults). The BMI of this patient was $20.5 \mathrm{~kg} / \mathrm{m}^{2}$ (4.5 SDS of BMI), her father's BMI was $26.5 \mathrm{~kg} / \mathrm{m}^{2}$. The other abnormal migration profile, newly described, was currently investigated. The BMI of this second patient was $19.2 \mathrm{~kg} / \mathrm{m}^{2}$ (3.2 SDS for BMI), her father's BMI was $27.7 \mathrm{~kg} / \mathrm{m}^{2}$.
P3-471 Fat Metabolism and Obesity

Orlistat Treatment in Obese Prepubertal Children

S. Norgren*1; P. Danielsson*1; M. Lötborn*1;R. Jurold*1.

C. Marcus ${ }^{1}$

${ }^{1}$ Huddinge Hospital, Stockholm, Sweden

Background: Pharmaceutical treatment of obesity in children is controversial due to risks of potential side effects. Objective: To investigate orlistat treatment in obese prepubertal children with regard to compliance, psychological well-being, and safety. Patients and Methods: Eleven healthy, obese prepubertal children 8.3-12.3 years, BMI SDS: 5.3-9.2) were treated with orlistat (Xenical $^{\circledR}, 120 \mathrm{mg}$ to each meal) for twelve weeks. Before treatment, a dietitian informed the participants and guardians regarding sources and recommended intake of dietary fat. The participants were evaluated by a psychologist and a pediatrician before and after treatment. Results: The summed weight loss of the participants was $48.9 \mathrm{~kg}$ with a median of $4.0 \mathrm{~kg}$ (range -12.7 to $+2.5 \mathrm{~kg}, \mathrm{p}=$ $0.016)$. The decreases in body weight and fat mass were strongly correlated ( $\mathrm{r}=$ $0.953, \mathrm{p}<0.01)$. Diarrhea was reported only after intake of high-fat products. Two children reported mildly increased flatulens. Increased avoidance of fattening food, and control of eating behavior $(p=0.011)$ were demonstrated by the Childrens Eating Attitudes Test. There was no sign of depression before or after treatment. After treatment, the children identified their own physique with a thinner silhouette $(\mathrm{p}=0.020)$, while the silhouette they aspired remained the same. In addition, they perceived loosing weight less difficult after treatment $(\mathrm{p}=0.043)$, and there was a trend toward increased motivation to loose weight. All children and guardians expressed a wish to continue the treatment after the study period. Conclusion: The participants could comply with and appreciated the treatment. Thus, the results prompt a larger long-term placebocontrolled study of the effectiveness of orlistat in the treatment of childhood obesity.
P3-470 Fat Metabolism and Obesity

\section{Anthropometric Predictors of Cardiovascular Risk Factors in Prepubertal Children: The Role of Waist Circumference \\ C. Maffeis ${ }^{* 1} ;$ A. Pietrobelli*1; A. Grezzani*1; S. Provera*1; ${ }^{*}$. Tatò ${ }^{1}$ \\ ${ }^{1}$ University of Verona, Verona, Italy}

Estimating body fat distribution might help to identify subjects with the highest risk of adverse lipid profile and hypertension. Waist has the advantage to be an easy and reproducible measure in the clinical practice. In order to explore the relationship between anthropometric variables, lipid concentrations and blood pressure we studied $845(\mathrm{M} / \mathrm{F}=1.1$; age: $3-11$ years) prepubertal children. The most of the correlations between body composition parameters, lipids and blood pressure were significant. ApoA1/B, HDL-ch, total cholesterol /HDL-ch, systolic blood pressure (SBP), diastolic blood pressure (DBP), adjusted for age, sex, weight and height (covariates) were significantly associated with waist, triceps and subscapular skinfolds using multivariate generalized linear model analysis. Four sets of multiple regression analyses were run, using ApoA1/ ApoB, HDL-ch, SBP and DBP as the dependent variables, respectively. Waist was used as a independent variable with age and gender as covariates. The four regression models were highly significant, accounting for $\approx 8 \%, 14 \%, 18 \%$, and $9 \%$ of the variance in ApoA1/ApoB, HDL-ch, SBP and DBP, respectively. Adding triceps or subscapular skinfold as an independent variable did not increase the prediction variance of the equations. The same analyses were run in a sub sample of 109 obese subjects (RBW > 120\%) confirming our findings with higher significance levels, despite of the lower number of subjects (accounting for $22 \%, 25 \%$ of the variance in ApoA1/ApoB and HDL-ch, respectively ). In conclusion, waist circumference may be a useful parameter to identify prepubertal children with adverse blood lipids and higher blood pressure.

\author{
P2-472 Diabetes and Glucose Metabolism \\ Increased Circulating Nitric Oxide in Young Patients \\ with Type 1 Diabetes mellitus and Persistent \\ Microalbuminuria: Relation with Glomerular \\ Hyperfiltration \\ F. Chiarelli ${ }^{1}$; S. Tumini ${ }^{1}$; L. di Ricco ${ }^{1}$; M. Pomilio ${ }^{1}$; A. Mohn ${ }^{1}$ \\ ${ }^{1}$ University of Chieti, Chieti, Italy
}

$\mathrm{NO}$ is candidate for mediating hyperfiltration and increased vascular pemeability induced by diabetes. Objective: Firstly, to evaluate whether serum NO levels and GFR values are increased in type 1 diabetic adolescents with and without persistent microalbuminuria; secondly, to assess if NO concentrations are influenced by chronic hyperglycaemia in these subjects. Finally, to study whether NO plays a role in the development of glomerular hyperfiltration and persistent microalbuminuria in type 1 diabetic patients with early nephropathy. Patients and Methods: Serum nitrite and nitrate $\left(\mathrm{NO}_{2}^{-}+\mathrm{NO}_{3}^{-}\right)$concentrations were assessed as index of NO production in 30 type 1 diabetic adolescents and young adults, 15 with and 15 without microalbuminuria (AER between 20 and $200 \mu \mathrm{g} / \mathrm{min}$ ) compared to a matched group of healthy controls. In all subjects glomerular filtration rate (GFR) was detemlined by radionuclide imaging (DTPA $\mathrm{TC}^{\mathrm{m}}{ }^{99}$ ). Results: NO serum concentrations and GFR values were significantly higher in microalbuminuric diabetic patients than in the other two groups. GFR resulted significantly and positively related to AER levels $\left(\mathrm{r}^{2}=0.75, \mathrm{p}<0.0001\right)$, whereas $\mathrm{NO}_{2}^{-}+\mathrm{NO}_{3}^{-}$serum content was independently associated with both AER and GFR values $(b=2.086, p=0.05, b=1.273, p=$ 0.0085 , respectively). Mean HbA1c serum concentrations was significantly higher in microalbuminuric than in normoalbuminuric diabetic subjects $(\mathrm{p}<$ 0.05 ) and was independently associated with AER values. HbAlc serum concentrations resulted significantly and positively related to $\mathrm{NO}_{2}+\mathrm{NO}_{3}$ serum content $\left(r^{2}=0.45, p=0.0063\right)$ and GFR values $\left(r^{2}=0.57, p=0.0011\right)$. Conclusions: In young type 1 diabetic patients with early nephropathy chronic hyperglycaemia is associated with an increased NO biosynthesis and action that contributes to glomerular hyperfiltration and persistent micro albuminuria. 
P2-473 Diabetes and Glucose Metabolism

Endothelial Perturbation in Children and Adolescents with Type 1 Diabetes: Role of the Immune-Inflammatory Reaction

M. Pomilio ; M. Romano ${ }^{4}$;S. Vigneri ${ }^{5}$; A. Falco ${ }^{2}$; P. Lelli Chiesa ${ }^{3}$; F. Chiarelli ${ }^{1} ;$ G. Davi $^{2}$

Department of Medicine, Division of ${ }^{1}$ Pediatrics, ${ }^{2}$ Hematology, and

${ }^{3}$ Pediatric Surgery, University of Chieti, ${ }^{4}$ Department of Human

Pathology, University of Messina; ${ }^{5}$ Department of Medicine,

University of Palermo, Italy

Vascular endothelin is a primary target of the unbalanced glycaemic metabolism in type 1 diabetes mellitus. Objective: To investigate on early events that could influence the development of diabetic angiopathy. Patients and Methods: We examined endothelial perturbation (both vWF:Ag and t-PA:Ag higher than 2SD above control values) in 40 young type 1 diabetic patients without retinopathy and/or nephropathy, subdivided according to the duration of diabetes in two groups. Group A (patients with diabetes duration no longer than 1 year) and group B (patients with diabetes duration longer than 1 year) were compared to a matched control group. Sixteen out of the 20 children belonging to group A were re-examined after 12 months. We also detennined $\mathrm{F}_{1+2}$, ICA and TNF- $\alpha$ levels, as index of prothrombotic state, immune and inflammatory response, respectively. Results: Group A children had a significant increase in vWF and tPA levels compared to either healthy subjects or group B patients. $70 \%$ of group A but only $20 \%$ of group B patients showed endothelial perturbation. Group A patients also showed increased levels of $F_{1+2}$, ICA and TNF- $\alpha$. Significant direct correlations between TNF- $\alpha$. and either $\mathrm{VWF}$ (Rho $=0.685$, $\mathrm{p}=0.0001)$, tPA $(\mathrm{Rho}=0.469, \mathrm{p}=0.0034)$ or $\mathrm{F}_{1+2}$ levels $(\mathrm{Rho}=0.61, \mathrm{p}=$ $0.0001)$ were observed. No statistically significant correlation was found between $\mathrm{HbA}_{1 \mathrm{c}}$ and either vWF, tPA or $\mathrm{F}_{1+2}$. After 1 year, 16 of the 20 group A patients had a significant reduction in $\mathrm{VWF}, \mathrm{tPA}, \mathrm{F}_{1+2}$ and TNF- $\alpha$ levels and 5 of these subjects the reversal of endothelial perturbation. Conclusions: Endothelial perturbation represents an early, and in some cases reversible, event in the chronology of type 1 diabetes in children. A correlation might exist between the initial immune-inflammatory reaction and the appearance of endothelial perturbation.

P2-474 Diabetes and Glucose Metabolism

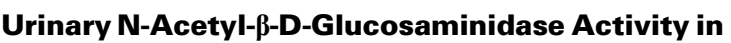
Childhood and Adolescents with IDDM: Relation to Albuminuria, Glycaemic Control and Puberty

B. Yüksel*1;M. Bakman*1;N.Ö. Mungan*1; G. Özer*1; Introduced by $P$. Cinas

${ }^{1}$ Cukurova University Medical Faculty, Adana, Turkey

Objective: The aim of this study was to assess the relationship between the urinary N-acetyl-beta-D-glucosaminidase (NAG) and urinary albumin excretion rate (AER), renal surface area, glycaemic control, puberty. Patients and Methods: This study was preformed in 44 children and adolescents with insulin dependent diabetes mellitus (IDDM), aged in 6-20 years with a disease duration from 2-11 years and in 44 age and sex matched healthy controls. The urinary NAG activity in morning urine in 36 diabetic patients and 14 controls was determined by colorimetric methods (Boehringer Mannheim, Germany). In all patients and controls we evaluated AER in overnight urine, which was measured by immunoturbidimetric methods. Microalbuminuria was defined as an AER above $20 \mu \mathrm{g} / \mathrm{min}$ and less than $200 \mu \mathrm{g} / \mathrm{min}$ in two of three overnight urine collections. Renal surface area was calculated by using kidney height and width, which were measured with ultrasonography. Results: In the diabetic patients urinary NAG/Cr ratios $(5.6 \pm 0.6 \mathrm{U} / \mathrm{gCr})$ were significantly higher than in controls $(1.6 \pm 0.1 \mathrm{U} / \mathrm{gCr})(\mathrm{p}>0.05)$. The urinary NAG/Cr ratios in the pubertal diabetic patients were significantly higher than in the prepubertal diabetic patients $(\mathrm{p}<0.05)$. No correlation was observed between urinary NAG/Cr ratio levels and sex, duration of disease, body mass index, HbAlc, glomerular filtration rate, blood pressure, AER, renal surface area. There was a significant correlation between AER and HbAlc, blood pressure, duration of disease, renal surface area. Microalbuminuria was detected in $15.9 \%$ of patients who were in pubertal stages III-V. Conclusions: Increased urinary NAG excretion in children and adolescents with IDDM doesn't show to be associated with early markers of diabetic nephropathy and glycaemic control. Puberty is involved in the appearance of microalbuminuria and in the elevations of urinary NAG in IDDM patients.
P2-475 Diabetes and Glucose Metabolism

Insulin Improves Clinical Status of Patients with Cystic Fibrosis Related Diabetes mellitus

A. Galli-Tsinopoulou ${ }^{1}$; S. Nousia-Arvanitakis*1; M. Karamouzis*1

Departments of ${ }^{1}$ Pediatrics and ${ }^{2}$ Biochemistry, Aristotle University Thessaloniki, Greece

Cystic fibrosis (CF) related diabetes mellitus (DM) is frequently underdiagnosed and associated with deterioration of the overall clinical status in $\mathrm{CF}$. Objective: The purpose of this study is to investigate the influence of insulin on nutrition, lung function and clinical status of CF patients. Patients and Methods: For a period of five years and at 6 monthly intervals body mass index $(\mathrm{BMI})$, spirometry $\left(\mathrm{FEV}_{1}\right.$ : \%), Schwachman score (SS), first phase insulin response (FPIR: IU/l) and $\mathrm{K}$ rate $(\% / \mathrm{min})$ were determined in $30 \mathrm{CF}$ patients (age range: 13 to 31 years) having exocrine pancreatic insufficiency. Results: During the study period six patients (age range: $15-22$ years, 3 males and 3 females) developed CF-DM (FPIR, mean \pm standard error: $23.9 \pm 1.97, \mathrm{~K}$ rate $0.51 \pm 0.02$ ) and required insulin therapy. During the prediabetic phase, there was marked deterioration of their nutritional and clinical status [BMI $\left.16.36 \pm 1.34, \mathrm{FEV}_{1} 50.66 \pm 6.68, \mathrm{SS} 66.33 \pm 3.84\right]$ which improved significantly shortly after the institution of insulin [BMI $19.07 \pm 1.06(\mathrm{t}=5.1694, \mathrm{p}=$ $0.0018), \mathrm{FEV}_{1} 70.83 \pm 5.4(\mathrm{t}=3.8183, \mathrm{p}=0.0062), \mathrm{SS} 84.50 \pm 4.41(\mathrm{t}=$ $6.6958, \mathrm{p}=0.0006)$ (paired t student's test)]. Positive correlation (Pearson) between FPIR and BMI $(r=0.759)$ was detected. Conclusions: A clear association of clinical status deterioration with insulin hyposecretion is demonstrated in CF. Insulin has a significant anabolic effect resulting in weight gain and improved pulmonary function. It is important to identify CF individuals at risk to develop DM by strict monitoring of glucose metabolism so that early insulin therapy is instituted.
P2-476 Diabetes and Glucose Metabolism

Analysis of the Factors Influencing Partial Remission in Children with Insulin Dependent Diabetes mellitus (IDDM)

F. Lombardo*1; M. Valenzise ${ }^{* 1} ;$ T. Arrigo ${ }^{1} ;$ F. De Luca ${ }^{1}$

1 Institute of Pediatrics, University of Messina, Messina, Italy

After diagnosis and insulin therapy onset a large proportion of IDDM children is known to pass into a phase of partial remission (PR), which has significant implications in terms of potential pharmacological and immune intervention. Aim of the present retrospective study was to evaluate the frequency of PR in 67 children aged between 1.4 and 17.3 years $(7.9 \pm 4.0)$ at the time of IDDM diagnosis and to analyze the factors influencing its duration. We abstracted insulin requirement data for the first 2 years after diagnosis (at 3, 6, 12 and 24 months) and defined PR as insulin requirement $<0.5 \mathrm{U} / \mathrm{kg} /$ day. PR duration was related to: a) patients' socioeconomic status; b) their clinical and metabolic status at the time of IDDM diagnosis (age, BMI, length of history of symptoms, blood $\mathrm{pH}$ and base excess, Hbalc concentrations, C-peptide levels 6 min after glucagon stimulation test); c) insulin requirements 3 months after diagnosis. In the entire cohort only 13 patients ( 4 aged less than 3 years at diagnosis) did not experience a PR. At 6 months 52 of cohort $(77.6 \%)$ were still in remission. By 12 and 24 months these figures had fallen to $41(61.2 \%)$ and $23(34.3 \%)$ respectively. The duration of PR correlated positively with: a) age at diagnosis ( $\mathrm{r}=$ $2.7, \mathrm{p}<0.05)$; b) BMI at diagnosis $(\mathrm{r}=3.1, \mathrm{p}<0.05)$; c) 6-min C-peptide levels $(\mathrm{r}=2.9, \mathrm{p}<0.05)$. A strong negative relationship was found between PR duration and insulin requirements at 3 months after diagnosis $(r=-0.77, p<$ 0.0001). No other variables significantly influenced PR duration. Conclusions: 1 ) more than $60 \%$ of our IDDM children experienced a prolonged ( $>12$ months) PR; 2) PR duration increased with age and BMI at diagnosis; 3) PR duration was positively influenced by the entity of residual B-cell function at diagnosis; 4) the greater is the reduction of insulin requirements soon after IDDM diagnosis, more prolonged is metabolic remission. 
P2-477 Diabetes and Glucose Metabolism

Presence of the IRS-1 G972R Variant in Children with a Glucokinase Mutation Is Associated to Differences in Insulin Secretion and Glucose Tolerance

O. Massa*1;I. Rabbone*2; R. Bonfanti*1; F. Barbetti*1; Introduced by M. Cappa

${ }^{1}$ IRCCS H S Raffaele, Milan, Italy; ${ }^{2}$ University of Turin, Turin, Italy

Mutations in the glucokinase (GCK) are the more common cause of Maturity Onset Diabetes of the Young (MODY) in Italian children. Fasting hyperglycemia (from 6.1 to $7.8 \mathrm{mmol}$ ) is a consistent metabolic feature of these patients in which, however, large variations in glucose levels at $120 \mathrm{~min}$ of OGTT (ranging from normal to diabetes) were observed. These differences are not readily explained as a direct consequence of the glucokinase mutation. Objective: The common variant G972R of the IRS-1 gene has been associated to subtle abnormalities of insulin secretion and action in vivo and in vitro. We searched for the presence of the R972 variant in children with MODY 2 mutations in order to evaluate the metabolic differenecs - if any - between simple MODY 2 mutants and double heterozygous (MODY 2/R972) individuals. Patients and Methods: The R972 variant was ascertained by RFLP-PCR in 71 children (47 GCK mutations) from different Italian families and in 56 of their affected adult relatives. Results: We identified twenty patients ( 11 children, 9 adults) bearing the R972 variant (15.7\%; 18 heterozygous, 2 children homozygous; MODY 2/R972). Mean fasting plasma glucose was similar in MODY 2 children with or without the R972 variant. Differently, MODY 2 bearing the R972 variant showed a higher mean plasma glucose levels at $120 \mathrm{~min}$ of OGTT when compared to MODY $2(10.0 \pm 2.0 \mathrm{vs} .8 .41 \pm 1.97 \mathrm{mmol} / 1)$. The first-phase insulin response $(1 \mathrm{~min}+3 \mathrm{~min})$ at the intravenous glucose tolerance test was impaired $(<1$ st percentile of normal Italian children) in 44\% of MODY $2(15 / 34)$ as compared to the $70 \%(7 / 10)$ of MODY2/R972. We are currently analysing metabolic data of affected relatives. Conclusions: These results indicate that the presence of IRS-1 R972 variant seems to influence the insulin secretion/action of individuals with MODY 2 mutations.

P2-478 Diabetes and Glucose Metabolism

\section{Type 2 Diabetes in U.K. Children - An Emerging \\ Problem \\ S. Ehtisham ${ }^{* 1} ;$ T. Barrett*1;N. Shaw ${ }^{1}$ \\ ${ }^{1}$ Birmingham Children's Hospital, Birmingham, United Kingdom}

Introduction: The prevalence of obesity in childhood is increasing and is a recognised risk factor for the development of diabetes. Children from ethnic minorities are now presenting with obesity and Type II diabetes. Aims: The aim of this study was to identify and characterise children with Type II diabetes in the Midlands. Methods: Children were identified by contacting paediatricians responsible for diabetes in five hospitals. Details were collected on demographics, mode of presentation, investigations and treatment on a standard proforma. Results: We identified eight girls aged 9-16 years who were of Pakistani, Indian or Arab origin. They were all overweight (percentage weight for height 140-206\%) and had a family history of diabetes in at least two generations. They presented insidiously with hyperglycaemia and glycosuria without ketosis and five were asymptomatic. Islet cell antibodies measured in six patients were negative. Four had acanthosis nigricans which is a cutaneous marker of insulin resistance and the other four had high plasma levels of insulin and/or C-peptide. These patients are distinct from those with maturity onset diabetes of youth (MODY) who do not show insulin resistance. All were managed with dietary measures. Seven were treated with a biguanide, three with sulphonylureas, one with an alpha-glucosidase inhibitor and two require insulin. Conclusions: We present the first UK case reports of Type II diabetes in children. Paediatricians need to be aware of the risk of Type II diabetes developing in childhood in high risk ethnic groups, particularly in association with obesity and a positive family history.
P2-479 Diabetes and Glucose Metabolism

Diabetic Cerebral Oedema, What Role Plays Taurine?

S. Rose*1;E. Davies*2; Introduced by M.O. Savage

${ }^{1}$ Heartlands Hospital, Birmingham, United Kingdom; ${ }^{2}$ University of

Birmingham, Birmingham, United Kingdom

Taurine is well known to act as an intracellular osmoregulator, especially in the central nervous system. In diabetic ketoacidosis neurones become hyperosmolar causing an influx of taurine. Objective: To determine whether during rehydration in diabetic rats there is a dislocation between water influx and taurine efflux causing cerebral oedema. Methods: Male Wistar rats were rendered diabetic using streptozotocin. They were sacrificed on day 6. One group was rehydrated with $15 \%$ of body weight 60 minutes before sacrifice. Brain taurine and water content were measured and compared to normal and rehydrated controls. Results: Brain taurine ( $\mathrm{mmol} / \mathrm{g}$ dry weight) was greater in diabetic rats than in controls, $24.6+0.81$ vs. $20.69+1.21(\mathrm{p}<0.05)$ There was no change with rehydration. Brain water content ( $\%$ total weight) was greater in controls than in diabetic rats, $84.63+1.29$ vs. $73.62+2.20$. Following rehydration there was a significant increase in brain water content in diabetic rats but not in controls 73.62 to $82.90(\mathrm{p}<0.01)$. Conclusions: These data are consistent with the hypothesis that taurine accumulates in the brain during hyperglycaemia and the lack of temporal correlation between taurine and water flux may be a factor in diabetic cerebral oedema. Modification of taurine flux may offer a possibility of prevention or treatment of this devastating complication of childhood diabetes.
P2-480 Diabetes and Glucose Metabolism

\section{Anti-Goat Immunoglobulin Antibodies in Adult Diabetic Patients \\ M. Haroun ${ }^{* 1}$; Introduced by M. Maes \\ ${ }^{1}$ University of Alexandria, Alexandria, Egypt}

Anti-goat antibodies in diabetic patients. Objective: To evaluate the antiruminant antibodies effect in diabetic patients. This protein-protein interaction interferes with immuno-precipitation assays leading to obscure the analysis of one specific type of protein found in human serum samples. Patients and Method: The study population consisted of 21 normal adult individuals (group 1), 21 diabetic patients (group 2) and the same diabetic individual serum samples purified from goat IgG (group 3) were used for analysis of $\mathrm{IgG}$ levels by ELISA and turbidity assay. Results: The results showed that the IgG found in goat bound to the diabetic serum immunoglobulins which in turn leading to an overestimation in the amount of immunoglobulins that the increase determined by turbidity assay was varied between $10 \%$ and $115 \%$. Initial results before purification from the interfering of goat antibodies suggested that diabetic patients had increment levels of IgG in their sera (group 2 have a high level of $\operatorname{IgG}$ while both group 1 and 3 have low levels of $\operatorname{IgG}$ ). It was found that control individuals had a mean $\mathrm{IgG}$ level of $9.1 \mathrm{mg} / \mathrm{ml}$ and diabetic individuals had a mean IgG level of $13.6 \mathrm{mg} / \mathrm{ml}$. There were significant increases in IgG level in diabetic patients $(\mathrm{p}<0.0005)$ while the mean level of $\mathrm{IgG}$ in diabetic patients after purification from goat IgG was $9.5 \mathrm{mg} / \mathrm{ml}$. Therefore there was no significant difference $(p<0.4)$ in IgG levels between normal and diabetic adults purified from goat IgG. Conclusion: There was an antiruminant antibody's interaction arised during the analyses of immunoglobulins, mainly IgG. This sort of interaction led to overestimation of immunoglobulin levels in diabetic patients which in turn produce unreliable results. 
P2-481 Diabetes and Glucose Metabolism

Subclinical Diabetic Neuropathy in Adolescent Type 1 Diabetics Diagnosed with the Rydel-Seiffer Tuning Fork

T.M. Kapellen*1, 2 ; A. Galler*1; W. Kiess ${ }^{1}$

${ }^{1}$ Children's Hospital, University of Leipzig, and ${ }^{2}$ University of

Giessen, Germany

Objective: In adults easily usable instruments like the Rydel-Seiffer tuning fork and the Semmes-Weinstein monofilament are widely used for screening of subclinical diabetic neuropathy. In children and adolescents these methods are not well established. Patients and Methods: 93 patients with type 1 diabetes ( 37 females, 56 males, aged 10-20 years) with a mean diabetes duration of 72 months (range 3-220 months) and a mean long term HbA1c of 7,8\% were compared to a group of 48 adolescent peers ( 25 females, 23 males, age matched). We compared scores for vibration sensation measured with the Rydel-Seiffer tuning fork and thermal sensation with the tip therm ${ }^{\circledR}$ (Axon GmbH, Germany). In all diabetic patients a detailed neurological examination including light touch perception with monofilament was performed. Results: 17 patients showed diminished reflexe response, absent reflexes were never seen. Slight signs of coordination problems were seen in 16 diabetic adolescents. Monofilament testing was normal in all patients. The examination of the thermal sensation revealed no differences between patients and controls. In contrast there were significant differences between patients and controls in vibration sensation at all measured sites (right 1 st metatarsale $(\mathrm{p}<0.001)$, left 1 st metatarsale $(\mathrm{p}<0.05)$, at the right malleolus medialis $(\mathrm{p}<0.0001)$ and at $2 \mathrm{nd}$ metacarpale of the right hand $(\mathrm{p}<0.0001))$. However, there was no correlation between vibration sensation and long-term metabolic control nor with blood glucose levels at examination nor with age or diabetes duration. Pathological vibration sensation defined as measurements under 2 standard deviations of the control were found in 23 diabetic patients $(25 \%)$. These diabetics had a longer mean diabetes duration than patients with normal vibration perception $(85 \pm 35 \mathrm{vs}$. $66 \pm 41$ months $(\mathrm{p}<0.05))$ but no differences in long term metabolic control. Conclusion: In this study only the measurement of vibration sensation with the Rydel-Seiffer tuning fork was able to define patients with subclinical diabetic neuropathy. This technique can easily be used in daily clinical practice to screen diabetic adolescents for early signs of neuropathy.

P2-482 Diabetes and Glucose Metabolism

Cystic Fibrosis (CF) Related Diabetes (CFRD): Clinical Consequences before Insulin Treatment and Management of the Insulin Therapy

M.A. Rolon*1;K. Benali*1;A. Munck*2;J. Navarro*2;A. Clement*3. N. Tubiana-Rufi*1; P. Czernichow ${ }^{1} ;$ M. Polak ${ }^{1}$

Departments of ${ }^{1}$ Pediatric Diabetology and ${ }^{2}$ Pediatric

Gastroenterology, Hôpital Robert Debré, and ${ }^{3}$ Department of

Pediatric Pneumology, Hôpital Trousseau, Paris, France

The prevalence of diabetes increases with increasing age of the CF patients. Diabetes may have in its preclinical phase a deleterious effect on the clinical status of the CF patients. Aim: To evaluate the repercussions of glucose intolerance on the nutritional state and respiratory function in a group of $\mathrm{CF}$ patients treated by insulin, in the period before this therapy was started. We also aim at describing the management of the insulin therapy and its effects in a french cohort of insulin treated CF patients (IT-CFRD). Patients and Methods: In a retrospective study, two groups were compared: group I, CF patients $(n=14)$ with diabetes, studied in the 5 years preceeding insulin therapy, and group II, matched CF patients $(n=14)$ with normal glucose tolerance (OGTT). The following parameters: body mass index (BMI), forced vital capacity (FVC), forced expiratory volume in 1 second $\left(\mathrm{FEV}_{1}\right)$ were collected during the same period in both groups. In the IT-CFRD group, parameters of insulin treatment (mean annual doses in units/kg/day), glycaemic control (mean annual HbAlc in \%) and frequence of hypoglycemia were collected during the five years following the onset of insulin therapy. Results: Before onset of insulin therapy, BMI, FVC and $\mathrm{FEV}_{1}$ values in the IT-CFRD deviated increasingly from those in the control patients. This decline in the clinical parameters of the IT-CFRD group reaches statistical significance in the 6- (for FVC and $\mathrm{FEV}_{1}$ ) or 3- (for BMI) month period preceeding the onset of insulin therapy. Insulin dosage increased from 0.62 (first year) to 1.25 after five years, in favor of a progressive decline of endogenous insulin production. Good metabolic control was achieved with $\mathrm{HbAlc}$ values varying from $6.6 \%$ to $7.8 \%$ (first and fifth year of treatment, respectively). BMI of the IT-CFRD returned to normal value after a year of treatment, demonstrating the impressive anabolic effect of insulin. Episodes of severe hypoglycemia were extremely rare: 2 over 42 years/patient observation time. The insulin regimen more often used was two daily injections of a mixture of short and intermediate acting insulin $(n=10)$ given with the insulin pen. Conclusion: The clinical status of the CF patients who will need insulin therapy deteriorates before the onset of the treatment. In the diabetic CF patients, insulin therapy allows an excellent anabolic effect and a good metabolic control with few hypoglycemia. Prospective randomized trial with small dosage of insulin during the prediabetic period in glucose intolerant CF patients is needed to study the benefit of early treament in the course of the disease.

\section{P2-483 Diabetes and Glucose Metabolism \\ Human Embryonic Pancreas Xenograft in NOD/SCID Mouse: A Model to Understand Human Pancreatic Development \\ M. Castaing $^{* 1}$; P. Czernichow ${ }^{1} ; R$. Scharfmann ${ }^{* 1} ;$ Introduced by M. Castaing}

1INSERM U457 Robert Debré Hospital, Paris, France

Aim: To define an experimental model allowing the study of pancreatic development in human. Methods: Human embryonic pancreases (6-10 weeks of gestation) were grafted under the kidney capsule of immunocompromized NOD/SCID mice. At different time points ( 7 days to 9 months), the grafts were removed. The presence and the maturity of the endocrine and the exocrine tissue were analyzed by immunochemistry. Results: The tissue increased in term of size in the NOD/SCID mice. Indeed, while the volume of the tissue was around $1 \mathrm{~mm}^{3}$ before the graft, it reached $1 \mathrm{~cm}^{3} 6$ months later. Both endocrine and exocrine tissue developed in this model. Before transplantation, exocrine cells were not detected, and endocrine cells were extremely rare, immature and found dispersed. After 3 months of grafting, a lot of exocrine cells (expressing lipase or trypsin) were detected. Moreover, a very important number of endocrine cells (around 10\% of the total cell number), expressing insulin or glucagon were detected. Those cells were found associated into islets of Langerhans. Moreover, the insulin cells that developed in the NOD/SCID mice seem mature, based on their expression of markers such as PDX-1, Nkx 6.1, GAD 65 , and the proconvertase PC 1/3. Conclusion: In this work, we have shown that human pancreatic development can occur when early embryonic pancreatic tissue is grafted in NOD/SCID mice. This experimental model will be useful to understand human pancreatic development. 
P2-484 Diabetes and Glucose Metabolism

\section{Neonatal Diabetes Mellitus: Clinical Description of a} Large Cohort of Permanent and Transient Cases

C. Metz ${ }^{* 1} ; B$. Gueguen-Giroux ${ }^{* 1} ;$ M. Polak ${ }^{2}$; on behalf of the NDM

French study group

${ }^{1}$ Department of Pediatrics, Hôpital Morvan, Brest; ${ }^{2}$ Pediatric

Endocrine Unit, Hôpital Robert Debré, Paris, France

Neonatal diabetes (NDM) is a rare but potentially devastating condition in a newborn. NDM has been essentially divided in two groups: the transient (TNDM) and the permanent (PNDM) form according to insulin dependence during the early course of the disease. Through a multicenter study by questionnaire in France, patients were included according to the following criteria: insulin treatment before three months of age, insulin therapy for more than 7 days and more than 18 months of survey. We identified a case as TNDM if insulin could be stopped within 18 months of its beginning. 32 cases were recruited according to these criteria: 15 TNDM and 17 PNDM. Intrauterine growth retardation (IUGR) was present in 24 (75\%) (TNDM, 14 and PNDM, 7). TNDM cases had significantly lower birth weight than PNDM cases (1820 \pm 451 versus $2444 \pm 636 \mathrm{~g} ; \mathrm{p}<0.05)$ for identical mean gestational ages $(38.5$ versus 39.2 weeks; $p=0.36$ ). Mean age at diagnosis was 9.2 days in TNDM versus 27.6 in PNDM $(\mathrm{p}<0.001)$. Mean blood glucose at diagnosis, was $5.07 \pm$ $3.15 \mathrm{~g} / \mathrm{l}$, ketonuria was present in $1 / 3$ of the cases and insulin levels were low (mean $7.6 \pm 6.16 \mathrm{mU} / \mathrm{l}$ ) in comparison with the glucose levels (no difference in glucose and insulin levels between both groups). No evidence of type I diabetes mellitus markers were found: anti-islets antibodies were negative (tested in 28) and HLA typing did not show type 1 DM related haplotypes (tested in 16). Pancreatic ultrasonography was normal in 15 subjects and detected a profound pancreatic hypoplasia in 1 case of PNDM. Initial treatment consisted in rehydration and insulin therapy. Mean initial insulin dose was 0.9 units $/ \mathrm{kg} / \mathrm{day}$ (0.16-2) and not different in TNDM and PNDM. Insulin therapy was then delivered either by continuous subcutaneous infusion (pump $n=13$ ) or by discontinuous insulin injections $(\mathrm{n}=21)$. Insulin therapy could be stopped in TNDM at a mean age of 5 months (0.5-15.3 months). Permanent hyperglycemia and insulin dependence occured in two cases of TNDM at 10 and 14 years of age (out of three cases of more than 8 years at the time of the survey). The mean observation time in TNDM is 31 months. Conclusion: even though in PNDM, IUGR is less often present and age at diagnosis is greater than in TNDM, it is impossible for the clinican to distinguish PNDM from TNDM in the neonatal period on a pure clinical basis. Recurrence is present after 'TNDM' and long term follow-up of these children is necessary. Advances in the genetic understanding of the disease may help to distinguish the two groups of patients in the neonatal period.

\section{P2-485 Diabetes and Glucose Metabolism}

Growth and IGFs in Children with IDDM at Onset of Disease. Evidence for Normal Growth, Age Dependency of the IGF System Alterations and Presence of a Small ( 18 kDa) IGFBP-3 Fragment in Serum

S. Cianfarani ${ }^{1}$; R. Bonfanti*2; M.-L. Manca Bitti*1; D. Germani*1; S. Boemi*3;G. Chiumello ${ }^{2} ;$ B. Boscherini*3

${ }^{1}$ Tor Vergata University, Rome, Italy; ${ }^{2}$ Scientific Institute

H 'San Raffaele', Milan, Italy; ${ }^{3}$ 'S.Eugenio' Hospital, Rome, Italy

Data on growth of children with IDDM prior to the onset of disease are conflicting and, though the IGF system has almost invariably been found altered at diagnosis, most of previous studies are affected by the small number of patients investigated. We have studied 60 IDDM children at onset of disease comparing their stature with target height, normal growth standards and height of 102 sexand age-matched controls. Furthermore, we have assessed serum IGF-I, IGF-II and IGFBP-3 levels and IGFBP-3 circulating forms. IDDM children were subdivided into two groups according to an age above $(n=26)$ or below $(n=34)$ six years. The values of endocrine variables of diabetics older than six were compared with those of 34 age-matched controls. Although height of diabetics was higher than growth reference values (mean height \pm SD: $0.64 \pm 1.4 \mathrm{z}$-score) and their target height (mean target height \pm SD: $0.1 \pm 0.84$ z-score, $p<$ 0.005 ), no significant difference in height was found between IDDM children and controls (mean height \pm SD: $0.64 \pm 0.95$ z-score) even analyzing the two age groups separately. HbAlc values were significantly higher in patients older than 6 years $(12.1 \pm 2.1 \%$ vs. $10.4 \pm 1.8 \%, p=0.001)$, and age at diagnosis correlated with $\mathrm{HbA1c}(\mathrm{r}=0.46, \mathrm{p}<0.001)$. Overall, IDDM children showed reduced levels of IGF-I (mean \pm SD: $-0.65 \pm 1.9 \mathrm{z}$-score), and normal levels of IGF-II (mean \pm SD: $-0.05 \pm 1.2$ z-score) and IGFBP-3 (mean \pm SD: $-0.06 \pm$ $1.2 \mathrm{z}$-score). However, whereas patients younger than 6 showed normal values of IGF-I, IGF-II and IGFBP-3, these peptides were significantly reduced in older subjects in comparison with either younger IDDM children or controls $(\mathrm{p}<0.01)$. IGFBP-3 immunoblot analysis revealed the presence of $\mathrm{a} \sim 18 \mathrm{kDa}$ fragment of IGFBP-3 in addition to the major $\sim 29 \mathrm{kDa}$ fragment and the intact form $(\sim 42-39 \mathrm{kDa})$ in $46 / 60$ IDDM patients, whereas the $\sim 18 \mathrm{kDa}$ band was absent in all 34 control sera. No relationship was found between the endocrine variables and stature at diagnosis. Diabetics with circulating $\sim 18 \mathrm{kDa}$ fragment showed increased IGFBP-3 proteolysis and IGF-I/IGFBP3 ratio, and reduced body mass index. Our results indicate that IDDM children at onset of disease are not taller than healthy peers and show that the IGF system is normal in younger diabetics and it deteriorates with age probably for the longer time elapsing between onset of disease and diagnosis in older subjects. Finally, we have demonstrated the presence of a small fragment of IGFBP-3 $(\sim 18 \mathrm{kDa})$ in serum of patients with more severely impaired nutritional status.

P2-486 Diabetes and Glucose Metabolism

\section{Hypoglycaemia and Elevated Urine Ethylmalonic Acid in a Child with Short-Chain Acyl-CoA Dehydrogenase 625G $\rightarrow$ A Gene Variation}

N.H. Birkebaek*1; N. Gregersen ${ }^{2}$; Introduced by K.E. Petersen

${ }^{1}$ Skejby Hospital, Aarhus, Denmark; ${ }^{2}$ Aarhus, Denmark

Elevated urinary ethylmalonic acid (EMA) is a marker of functional deficiency of short-chain acyl-CoA dehydrogenase (SCAD). In patients with EMA aciduria we have shown a homozygous prevalence of $60 \%$ of the SCAD gene variation $625 \mathrm{G} \rightarrow \mathrm{A}$, resulting in a variant Gly $185 \mathrm{Ser}$ SCAD protein with reduced stability compared to the control SCAD protein. As SCAD is a key enzyme in the oxidation of fatty acids, which serve as substrates for gluconeogenesis, reduced SCAD activity may predispose for fasting hypoglycaemia. We report on a 14 months old Danish boy, who after few days fever and an upper airway infection presented with hypoglycaemia. He had previously been healthy with normal psychomotoric development. On admission blood glucose was $2.1 \mathrm{mmol} / \mathrm{l}$ and base excess -11 . After two days on normal food he was clinically and biochemically normalised. An overnight fasting blood glucose was $3.0 \mathrm{mmol} / \mathrm{l}$. A thorough biochemical examination did not reveal the cause of hypoglycaemia. No DNA mutation in the medium chain acyl-CoA dehydrogenase (MCAD) gene was found, but homozygosity for the SCAD gene variation $625 \mathrm{G} \rightarrow \mathrm{A}$, was demonstrated. The EMA concentration in two morning urine samples were 15 and $24 \mu \mathrm{mol} / \mathrm{mmol}$ creatinine, indicating mild EMA aciduria (controls less than $10 \mu \mathrm{mol} / \mathrm{mmol}$ creatinine). The child was treated with frequent meals. Twenty months old he was admitted due to gastroenteritis. After $200 \mathrm{ml}$ juice, blood glucose was $4.3 \mathrm{mmol} / \mathrm{l}$, base excess was -7 , and EMA was $41 \mu \mathrm{mol} / \mathrm{mmol}$ creatinine. His father was homozygous for the $625 \mathrm{G} \rightarrow \mathrm{A}$ gene variation, while his mother was heterozygous. The father informed us that when fasting for hours he sometimes felt weak, tired and very hungry. Symptoms disappeared after a meal. In contrast to the known risk of severe fasting hypoglycaemia in patients with MCAD deficiency, SCAD deficiency is less well characterised. In our patient reduced function of the SCAD protein may result in intracellular accumulation of the cytotoxic butyric acid, which is converted to, and excreted in urine as EMA. Further, gluconeogenesis is compromised due to lack of substrates from oxidation of short-chain fatty acids in the fasting state; this may explain the predisposition for fasting hypoglycaemia in our patient. 
P2-487 Diabetes and Glucose Metabolism

\section{Mitochondrial Bioenergetics in Circulating} Lymphocytes from Type 1 Diabetic Patients

G. Lenaz ${ }^{* 1} ;$ G. Sgarbi*3; M.D'Aurelio*1; M. Cavazzoni*1. A. Bernacchia*1; A. Biondi*1; C. Bovina*1; G. Biagini*3; L. Ragni*2.

S. Salardi ${ }^{2}$;E. Cacciari ${ }^{2}$; Introduced by E. Cacciari

Departments of ${ }^{1}$ Biochemistry and ${ }^{2}$ Pediatrics, University of

Bologna, ${ }^{3}$ Institute of Morphology, University of Ancona, Italy

Mitochondrial alterations in diabetic patients in peripheral tissues are possible consequences either of insulin deprivation or of secondary effects of oxidative stress. Objective: Lymphocytes are a good model to study mitochondrial function in diabetes, since modification of energy supply might explain the immunological impairment in diabetic patients. Patients and Methods: Twenty IDDM patients aged from 9 to 21 years and 20 age-matched controls were used. They were under insulin treatment; however, most were hyperglycemic at the time of blood withdrawal and all had values of glycated hemoglobin higher than normal. Peripheral blood lymphocytes were isolated and respiration was measured by a micro-oxygen electrode either in intact cells or in digitonin-permeabilized cells. Mitochondrial membrane potential $\Delta \Psi$ mit was measured in intact cells by flow cytometry. Plasma membrane oxidoreductase (PMOR) was measured with endogenous NADH and the dye DCIP as acceptor. Results: Respiratory activities in permeabilized cells, normalized to cytochrome oxidase, using both NAD-linked substrates and succinate, as well as glucose-supported respiration in intact cells, were decreased in patients. The $\Delta \Psi$ mit was normal, but in patients it exhibited higher sensitivity to uncoupling, so that the slope of a plot of uncoupling vs. uncoupler concentration was significantly increased. The finding is interpreted as a decreased input of protons by the respiratory chain. The PMOR activity was increased in patients, suggesting its up-regulation in order to release cytosolic NADH accumulated as a consequence of mitochondrial impairment, and possibly to reduce quinones to their antioxidant forms. Conclusion: Most changes observed appear to depend on the state of oxidative stress accompanying diabetes.

P3-488 Diabetes and Glucose Metabolism

\section{Does Humoral Immunity against Insulin (Insulin Autoantibodies, IAA) Promote Lipodystrophy in Children and Adolescents with Type 1 Diabetes? \\ K. Raile ${ }^{* 1} ;$ V. Noelle*2; R. Landgraf*3; H.P. Schwarz ${ }^{2}$ \\ ${ }^{1}$ University Children's Hospital, Munich; ${ }^{2}$ Munich; ${ }^{3}$ University of Munich, Germany}

Introduction: In children and adolescents with type 1 diabetes the development of lipodystrophy, i.e lipohypertrophy and lipoatrophy, are common problems at frequently used injection sites. Especially lipohypertrophic areas cause irregular and prolonged absorption of injected insulin and therefore lead to high fluctuations of blood glucose. So far, the underlying mechanism for the formation of lipodystrophy is not clear. Lipohypertrophy consists of both fibrous and fat tissue and growth is explained by local trophic effects of injected insulin. Lipoatrophy is rarely seen in children, and its formation is explained by local immunological reactions against fat and fibrous tissue. Patients and Methods: In 81 randomly surveyed children and adolescents with type 1 diabetes on regular injections of insulin antibodies, specific for type 1 diabetes (IAA, IA-2, GAD65) and clinical parameters were recorded. To classify lipodystrophy, a clinical score was used as follows: grade $0=$ no hypertrophy of the injection site; grade $1=$ moderate hypertrophy of fat and no increased density of tissue; grade 2 = increased density of tissue; grade $3=$ lipoatrophy. Results: Out of 81 examined children, 2 had lipoatrophy (grade 3 ), 10 severe lipohypertrophy (grade 2), 17 moderate (grade 1) lipohypertrophy and 52 no alteration (grade 0 ) of at least one injection site. Markedly elevated IAA levels were present in patients with grades 2 and 3 compared with patients with grades 0 and 1 lipodystrophy $(\mathrm{p}=0.017$, t-test). No significant difference between both groups was found regarding age, duration of diabetes, insulin dose and episodes of severe or moderate hypoglycaemia. Linear regression analysis displayed a significant correlation between the levels of IAAs and the degree of lipodystrophy $(\mathrm{p}=0.005)$. IA-2 levels correlated negatively with duration of diabetes $(\mathrm{p}=0.005)$ and GAD antibodies showed no correlation at all. Conclusions: The correlation of lipodystrophy and IAA levels suggests involvement of humoral immunity against injected insulin in the development of lipodystrophy. This process is independent from autoimmune beta cell inflammation, reflected by levels of IA-2 and GAD antibodies. Nevertheless, the causal link between IAA levels and lipodystrophy remains unproven and stipulates further longitudinal studies.
P3-489 Diabetes and Glucose Metabolism

\section{Ten Years Experience in Management of Diabetic Ketoacidosis and Ketosis: 140 Episodes at the Paediatric Age}

N. Yordam ${ }^{1}$; E.N. Gönc *1

${ }^{1}$ Hacettepe University, Ankara, Turkey

Diabetic ketoacidosis (DKA) is still a cause of morbidity and mortality in childhood. The unconsciousness at presentation is a sign of severity in DKA. Whatever the exact risk factors for unconsciousness are in debate. Objective: To elucidate the clinical and laboratory risk factors of unconsciousness on admission and to analyse our ten years experience on DKA and diabetic ketosis (DK) treatment protocol as far as the morbidity and mortality are concerned. Patients and Methods: The clinical and laboratory findings on admission and during therapy including degree of dehydration, consciousness and serum glucose, ketone, $\mathrm{pH}$, bicarbonate, blood urea nitrogen, electrolytes of 140 episodes (96 DKA, $44 \mathrm{DK}$ ) of 112 patients ( 58 males, 54 females) were retrospectively analysed. Following one to two hours of saline infusion, all patients were treated by i.v. insulin $(0.05-0.15 \mathrm{U} / \mathrm{kg} / \mathrm{h})$ and fluid consisting of $2.5 \%$ dextrose and $75 \mathrm{mEq} / 1 \mathrm{NaCl}(280 \mathrm{mOsm} / \mathrm{l})$. Results: Mean age of $97(69.3 \%)$ new onset diabetes cases was $9.11(4.32)$ years $(1.1-16.3)$ whereas it was $12.93(2.85)$ years $(4.8-19.2)$ in 43 established diabetes cases. Unconsciousness at presentation was not observed in DK cases but $31 \%$ of DKA cases. Levels of consciousness of the patients were negatively correlated with serum bicarbonate and positively correlated with the degree of dehydration, serum ketone and glucose levels. Bicarbonate level less than $15 \mathrm{mmol} / \mathrm{l}$ was not found to be a risk factor for the existence of unconsciousness. There was no correlation between consciousness and serum effective osmolality. Effective osmolality more than 320 $\mathrm{mOsm} / \mathrm{kg}$ was not found to be a risk factor for the development of unconsciousness. Means of duration of i.v. insulin infusion were 15.18 (7.22) and 7.70 (3.81) hours in DKA and DK cases respectively. Deterioration or loss of consciousness as well as mortality during treatment were not observed. Hypoglycaemia was seen in three and hypocalcaemia in one case. Conclusions: Biochemical analysis of 140 episodes revealed that at admission serum effective osmolality did not appear to be a predictive factor for the presence of unconsciousness. Whereas the severity of dehydration, acidosis, ketosis and hyperglycaemia correlated positively with the level of unconsciousness. The absence of clinical brain oedema during treatment seemed to be related with the composition of the i.v. fluid.

P3-490 Diabetes and Glucosè Metabolism

\section{Is the Composition Different in Prepubertal Diabetic Children from Their Healthy Counterparts?}

F. Gözübenli*1; i. Arslanoğlü ${ }^{* 1} ; F$. Çiloğlu*2; P. Isgüven ${ }^{1}$; M. Yıldız*1; S. Ozçay ${ }^{1}$

${ }^{1}$ Division of Pediatric Endocrinology, Göztepe Educational Hospital

of SSK, and ${ }^{2}$ Marmara University Sports Academy, Istanbul, Turkey

Little is known about the body composition in childhood diabetes and which method is appropriate for its determination. Objective: To evaluate body composition in prepubertal nonobese diabetic children and to compare the value of some methods for clinical use. Patients and Methods: We studied bioelectrical impedance analysis (BIA) and compared it with other fatness parameters, i.e. body mass index (BMI), skinfold thickness (ST, biceps, triceps, subscapular, suprailiac, femoral and their sum), body circumference (upper arm, femoral, waist, hip, waist/hip) measurements and serum leptin in prepubertal Type 1 diabetic children and healthy controls. Twenty-five patients with diabetes duration $>2.5$ years were matched by age, sex and BMI with 24 healthy controls (age $8.6 \pm 1.5$ vs. $8.3 \pm 1.3$ years, M/F 14/11 vs. 13/11, BMI $16.4 \pm 1.5$ vs. $15.6 \pm 1.5 \mathrm{~kg} / \mathrm{m}^{2}$ respectively). Results: Total ST and waist circumference were significantly increased in the patient group in comparison to the normal controls ( $p=0.008$ and 0.02 respectively). Fat mass $\%$ in BIA however showed large intragroup variation, and no significant intergroup difference occurred $(p=0.5)$. Leptin was significantly correlated with BMI, total ST, waist circumference and BIA $(\mathrm{p}<0.001,0.001,0.01$ and $=0.012$ respectively). Conclusions: The problem of obesity as defined increased body fat occurs even in diabetic children with normal BMI before puberty, but BIA is not helpfull enough in its determination whereas carefull skinfold thickness and body part circumference measurements were found superior to BIA. 
P3-491 Diabetes and Glucose Metabolism

Metabolic Control and Insulin Regimens in Young Danish Patients with Diabetes

B.S. Olsen ${ }^{* 1}$; H.B. Mortensen ${ }^{1}$; P. Hougaard*2;

${ }^{1}$ Glostrup University Hospital, Glostrup, Denmark; ${ }^{2}$ Novo Nordisk,

Bagsværd, Denmark

A Danish nationwide cohort of children and adolescents was followed up for 6 years (1989-1995) to evaluate the change in metabolic control and insulin regimen over time. In total 353 patients $(50.1 \%$ of the inception cohort) were included in both study years. In 1995 mean age was 20.7 years and mean diabetes duration was 13.2 years. Mean HbAlc was 9.5\% in 1989 (normal range 4.3-5.8) and $9.7 \%$ in 1995 . HbAlc increased to $10 \%$ between the ages of 12 to 20 years followed by a gradual decrease to $8.6 \%$ after 25 years of age. In 1989 $55.2 \%$ of the patients were treated with 3 or more daily insulin injections compared to $87.5 \%$ in 1995 . Among the patients treated with 1 or 2 injections in $1989,79 \%$ increased the number of daily insulin injections in 1995 , while $21 \%$ remained on the same regimen. Among the patients treated with 3 or more injections in $1989,10 \%$ received more daily injections, $76 \%$ remained on the same regimen while $14 \%$ received fewer injections in 1995 . Mean daily insulin dose was $1 \mathrm{IU} / \mathrm{kg} / 24 \mathrm{~h}$ at the age of 15 years and subsequently decreased to 0.75 $\mathrm{IU} / \mathrm{kg} / 24$ hours at the age of 25 years. Between 15 and 20 years girls received more insulin than boys while males tended to receive more insulin from the age of 20 years. Females had significantly higher BMI than males and even the age of 25 years the BMI values of both sexes continued to increase. Up to the age of 16 years about half of the young patients were treated with pre-mixed insulin (all types), the other half with a mixture of intermediate and shortacting insulin. After the age of 16 years more than $90 \%$ of the patients had a mixture of short and intermediate-acting insulin. Among the patients on twice insulin daily, $89 \%$ were treated with premixed insulin, either alone or in combination with intermediate-acting insulin, while $53 \%$ of the patients on 3 daily injections were treated with pre-mixed insulin in combination with short- and intermediate-acting insulin. Of the patients on 3 or 4 injections $98.5 \%$ received a mixture of short- and intermediate-acting insulin. Metabolic control is still unsatisfactory in young Danish patients with diabetes. Interestingly, the poor metabolic control seem to continue into young adulthood. In the treatment centres intensified insulin regimens are used particularly in the older adolescents. Nevertheless the goal of near normoglycaemia is achieved in only a few. This suggest that factors such as patient-education and self-care behaviour may be of greater importance for improved metabolic regulation than insulin regimens.

P3-492 Diabetes and Glucose Metabolism

\section{Leptin in Children and Adolescents with Type 1}

Diabetes: A 1 Year Longitudinal Study

R. Bonfanti*1; P. Brambilla2 ; S. de Poli2 ; S. Mora ${ }^{2}$; E. Calzi ${ }^{2}$.

E. Bognetti ${ }^{2}$; F. Meschi' ${ }^{2}$;. Barbieri2 ; G. Chiumello ${ }^{2}$

1 Università degli studi di Milano, Milan, Italy; ${ }^{2}$ Clinica Pediatrica III,

Milan, Italy

Type 1 diabetic patients show an important variation in body weight, during the first year of insulin treatment. Insulin is directly and undirectly involved in the regulation of leptin secretion. However, discordant results have been described in patients at onset and during the first year of diabetes. Aim of this study was to evaluate the correlations between auxologic and metabolic parameters and leptin variations in a cohort of patients with type 1 diabetes mellitus at onset and during the first year of treatment. From a cohort of 300 diabetic patients followed regularly during the first year of disease, we investigate 26 patients $(14 \mathrm{M} / 12 \mathrm{~F})$, with a mean age $12 \pm 2.5$ yrs (range $6-15.8) .10$ patients presented at onset a weight loss ( rbw $<85 \%)$ and 16 patients a normal weight (rbw 85-115\%). We evaluated metabolic parameters (HbAlc, pH, C-peptide, insulin dose), auxologic parameters (weight, height, relative body weight, height sds, bmi) at the onset and at 6 and 12 months of insulin treatment. Leptin was measured by radioimmunoassay at onset of diabetes within 6 days from first insulin administration and after correction of DKA if present at each time points of follow up. Results: At onset, log transformed leptin levels, normalized by bmi, sex, age, were in the lower part of normal reference range $\left(3-50^{\circ}\right.$ centile), mean $0.54 \pm 0.29 \mathrm{ng} / \mathrm{ml}$. Leptin levels showed a decrease at 6 months and increased thereafter (at 6 months $0.49 \pm 0.3$, at 12 months $0.56 \pm$
$0.34, p=0.01$, Anova). Leptin levels showed a significant correlation between each others at each time point. Leptin levels were not different between patients who did and did not gain weight during follow up (respectively 0.6 vs. $0.45,0.54$ vs. $0.41,0.6$ vs. $0.42 \mathrm{ng} / \mathrm{ml}$ ). There were no correlations between variation in leptin levels and variation in bmi. Leptin values were correlated significantly to bmi, insulin dose and sex at onset and not to degree of acidosis, and at 12 months of disease. However, at 6 months leptin levels were related to bmi but also to residual beta-cell function (C-peptide) and degree of metabolic control ( $\mathrm{HbAl}$ ). Conclusion: We confirm that in IDDM patients at onset of disease leptin levels are in the normal range, and then showed a decrease at 6 months followed by an increase thereafter. Leptin levels are mainly explained by BMI and sex variation as in normal subjects, but at 6 months exists a negative relation to residual insulin secretion, which can explain the decrease in leptin levels. These data suggest a role of $\mathrm{C}$-peptide in the regulation of leptin secretion, and this new issue needs to be confirmed by larger studies.

P3-493 Diabetes and Glucose Metabolism

Relationship between Early Feeding Habits and Childhood Type 1 Diabetes mellitus during 1995-1999 in Sweden and Lithuania

V. Sadauskaite ${ }^{* 1} ;$ E. Jasinskiene ${ }^{* 1} ;$ Z. Padaiga*1; U. Samuelsson*2 ; J. Ludvigsson*2

${ }^{1}$ Kaunas University of Medicine, Kaunas, Lithuania; ${ }^{2}$ Linköping

University Hospital, Linköping, Sweden

Background: A relationship between type 1 diabetes mellitus (DM) and other immune diseases has been described. Early introduction of flour, containing gluten, in relation to coeliac disease and early introduction of egg, a cause for atopic status, are interesting as potential risk factors in causal relationship with type $1 \mathrm{DM}$ in children. Early introduction of meat as a source of nitrites that increase risk for type $1 \mathrm{DM}$ could also influence development of type $1 \mathrm{DM}$ later in life. Aim: To compare differences in feeding habits between children with diabetes and healthy controls in Sweden and Lithuania, where incidence of childhood type 1 DM is quite different (1997: 31.9 and 10.3/100 000, respectively). Methods: A case-control study involved all newly diagnosed children, 0-15 years, with type 1 DM (as cases) in South-East of Sweden during 1995$1999(\mathrm{n}=403)$ and in Lithuania during 1996-1999 $(\mathrm{n}=234)$. Three age and sex matched randomly selected population based healthy children were invited as controls. Control response rates were $74.8 \%$ in Sweden and $84.6 \%$ in Lithuania. Information on risk factors important for the development of type $1 \mathrm{DM}$ was collected using a structured questionnaire at the time of diagnosis in both countries. Results: There were no differences in the time of introduction of flour and egg between children with diabetes and controls in both countries, except for meat, which was introduced significantly later among healthy children in both countries altogether and in the 5-9 years age group in Sweden and in the 5-9 years and 10-15 years age groups in Lithuania.

Table. Month of introduction of food items in age groups

\begin{tabular}{lllll}
\hline & $\begin{array}{l}\text { 0-4 years } \\
\text { cases/controls }\end{array}$ & $\begin{array}{l}\text { 5-9 years } \\
\text { cases/controls }\end{array}$ & $\begin{array}{l}\text { 10-14 years } \\
\text { cases/controls }\end{array}$ & $\begin{array}{l}\text { Total } \\
\text { cases/controls }\end{array}$ \\
\hline $\begin{array}{l}\text { Sweden } \\
\text { Flour }\end{array}$ & $6.2 / 6.3$ & $5.7 / 6.3$ & $5.8 / 5.6$ & $5.8 / 6.0$ \\
Egg & $8.7 / 8.7$ & $8.9 / 8.7$ & $7.4 / 7.6$ & $8.4 / 8.3$ \\
Meat & $6.6 / 6.6$ & $6.6 * / 7.1$ & $6.9 / 6.7$ & $6.7 * / 6.8$ \\
& & $(\mathrm{p}=0.003)$ & & $(\mathrm{p}=0.004)$ \\
\hline Lithuania & & & & \\
Flour & $4.8 / 5.4$ & $5.6 / 5.5$ & $5.4 / 5.2$ & $5.4 / 5.3$ \\
Egg & $5 . / 6.2$ & $5.2 / 5.5$ & $5.4 / 5.7$ & $5.4 / 5.7$ \\
Meat & $6.5 / 6.7$ & $6.4 * / 6.6$ & $6.2 * / 6.4$ & $6.3 * / 6.5$ \\
& & $(\mathrm{p}=0.013)$ & $(\mathrm{p}=0.002)$ & $(\mathrm{p}=0.001)$ \\
\hline
\end{tabular}

Conclusions: Early introduction of meat products might contribute to a risk for development of type 1 DM later in life both in Sweden and Lithuania. 
P3-494 Diabetes and Glucose Metabolism

Antibodies to Tissue Transglutaminase $\mathbf{C}$ in Newly-Diagnosed and Long-Standing Type 1 Diabetes

R. Lorini 1 ;.A. Avanzini*2; E. Lenta*2; M. Cotellessa*1; C. De Giacomo*2; P. Barbieri*1; G. d'Annunzio ${ }^{* 2}$

${ }^{1}$ Department of Pediatrics, University of Genoa, Gaslini Institute,

Genoa; ${ }^{2}$ Department of Pediatrics, IRCCS Policlinico San Matteo,

Pavia, Italy

Tissue transglutaminase C (tTG) has been identified as an autoantigen target of antiendomysium antibodies (EMA), the serological marker of coeliac disease (CD). We detected tTG-IgA by immunoenzymatic method, IgA antiendomysium (EMA) by immunofluorescence, and serological markers of type 1 diabetes (IA-2A and GADA) by RIA in 68 type 1 diabetes patients ( $36 \mathrm{M}$ and $32 \mathrm{~F}$, aged 1-25.7 yrs, 31 newly-diagnosed and 37 with disease duration 1.1-16 years). TTG values $>14 \mathrm{U} / \mathrm{ml}$ (mean $+3 \mathrm{SD}$ of 56 controls) were defined as positive. In 18/68 patients (6/31 newly-diagnosed, ND and 12/37 long-standing, LS) high levels of tTG were observed, with a frequency higher than controls $(0 / 56)(\mathrm{p}=0.0000)$. In $2 / 6 \mathrm{ND}$ patients positive for tTG EMA were also found, and jejunal biopsy revealed CD. In 10/12 LS patients positive for tTG EMA were also found, and jejunal biopsy showed CD in 9 and partial villous atrophy ('latent' CD) in 1. Results of immunological markers of diabetes are reported in the table. TTG have been suggested as a sensitive test replacing EMA detection for diagnosis and screening of CD. Since multiple immunological abnormalities have been reported at clinical diagnosis of diabetes, the detection of tTG in newly-diagnosed patients could reflect an abnormal immunological response in the early stage of diabetes, with increased production of antibodies by specific clones of B-lymphocytes, instead of markers of coeliac disease.

\begin{tabular}{|c|c|c|c|c|c|c|}
\hline \multirow[t]{2}{*}{ Antibodies } & \multirow[t]{2}{*}{$\begin{array}{l}\text { ND pts } \\
(\mathrm{n}=31)\end{array}$} & \multirow[t]{2}{*}{$\begin{array}{l}\text { LS pts } \\
(\mathrm{n}=37)\end{array}$} & \multicolumn{2}{|c|}{ EMA positive } & \multicolumn{2}{|c|}{$\begin{array}{l}\text { Abnormal } \\
\text { biopsies* }\end{array}$} \\
\hline & & & ND & LS & ND & LS \\
\hline TTGA-GADA-IA2A & 3 & 5 & 1 & 5 & 1 & 5 \\
\hline TTGA-GADA & 1 & 5 & 0 & 3 & - & 3 \\
\hline TTGA-IA2A & 1 & 1 & 0 & 1 & - & 1 \\
\hline GADA-IA2A & 12 & 4 & 0 & 0 & - & - \\
\hline TTGA & 1 & 1 & 1 & 1 & 1 & 1 \\
\hline GADA & 4 & 2 & 0 & 0 & - & - \\
\hline IA2A & 4 & 6 & 0 & 0 & - & - \\
\hline No antibodies & 5 & 13 & 0 & 0 & - & - \\
\hline
\end{tabular}

* Only EMA positive patients were biopsied.

P3-495 Diabetes and Glucose Metabolism

Molecular Genetic Analysis in 230 Families with MODY. An Italian Experience

R. Lorini ${ }^{1}$; G. d'Annunzio*2; A. Alibrandi*2; M. Cotellessa*1 L. Vitali*2 $^{*}$;P. Barbieri*1; O. Massa*3; I Carbone ${ }^{* 1}$;E. Bonifacio*3 C. Bellanné-Chantelot ${ }^{*} ;$ C. Klersy*5; F. Barbetti*3

${ }^{1}$ Genoa University, Genoa, Italy; ${ }^{2}$ Pavia University, Pavia, Italy;

${ }^{3}$ IRCCS HS. Raffaele, Milan, Italy; ${ }^{4}$ Fondation Jean Dausset-CEPH,

Paris, France; ${ }^{5}$ IRCCS Policlinico S.Matteo, Pavia, Italy

Maturity-onset diabetes of the young (MODY) is an autosomal dominant form of non-insulin dependent diabetes with onset before $25 \mathrm{yr}$ of age. In 1994 we started a study of MODY in Italy. The screening procedures include immunologic, metabolic and genetic investigations according to the flow-chart for incidental hyperglycaemia established by the Italian Pediatric Diabetes Study Group. Until January 2000 we registered 250 subjects with clinical diagnosis of MODY, including familial type 2 diabetes, consistent with an autosomal dominant inheritance, and without type 1 diabetes autoimmunity. After a complete screening of the glucokinase gene (MODY2) in 180 families and an almost complete screening in another 50 families, 89 MODY2 mutations were found. In contrast a complete screening of the HNF-1 $\alpha$ gene (MODY3) in 46 families and an almost complete screening in another 110 families led to the identifica- tion of only 9 MODY3 mutations. In 35 of these subjects the presence of MODY4 was excluded. Regarding metabolic evaluation, among MODY2 subjects tested OGTT was normal in 29/79 (37\%), impaired in 37/79 (47\%) and diabetic in 13/79 (16\%); FPIR was below the first percentile in 17/75 (23\%). Among MODY3 subjects tested OGTT was normal in 3/7 (43\%) and diabetic in $4 / 7$ (57\%), FPIR was below the first percentile in 4/9 (44\%). Our results underline the importance of investigating children with incidental hyperglycaemia and familial type 2 diabetes, consistent with an autosomal dominant inheritance, without type 1 diabetes autoimmunity. Moreover, genetic analyses revealed that mutations in glucokinase gene are the most frequent cause of MODY in Italy.

\section{P3-496 Diabetes and Glucose Metabolism \\ Normal Regulation of Insulin Secretion in the Carriers of an Inactivating $\boldsymbol{\beta}$-Cell K-ATP Channel Mutation \\ H. Huopio*1;I. Vauhkonen*1; J. Komulainen ${ }^{1 ;}$ L. Niskanen*1; \\ T. Otonkoski2 ; M. Laakso ${ }^{1}$; Introduced by T. Otonkoski \\ ${ }^{1}$ Kuopio University Hospital, Kuopio, Finland; ${ }^{2}$ Helsinki, Finland}

Recessive autosomal mutations in the sulfonylurea receptor SUR 1 gene are the major cause of congenital hyperinsulinemia (CHI). Despite of the hereditary nature of $\mathrm{CHI}$, detailed studies of glucose metabolism in heterozygotes have not been reported. To investigate this we determined the glucose metabolism of eight parents of $\mathrm{CHI}$ patients, heterozygous for the K-ATP channel inactivating SUR1 mutation V187D, and ten matched control individuals. Oral and intravenous glucose tolerance tests and both hyperinsulinemic euglycemic and hypoglycemic clamp studies were performed. In addition, we used a symptom questionnaire to evaluate the subjective symptoms during the hypoglycemic clamp study. The results show that there are no differences between the V187D carriers and controls in parameters of insulin secretion or glucose tolerance. The counterregulatory hormone responses during hypoglycemia did not differ either. The only difference between the groups was the better awareness of hypoglycemic symptoms in the V187D-carriers. Our results conclude that heterozygosity for SUR1 mutation causing the recessively inherited $\mathrm{CHI}$ does not impair the regulation of insulin secretion or tissue sensitivity to glucose.

P3-497 Diabetes and Glucose Metabolism

Is Increased Body Fat a Risk Factor in Prepubertal, Nonobese Diabetic Children?

i. Arslanoğlu*1;F. Gözübenli*1; P. Issgüven*1;M. Yıldız*1;

S. Ozçay*1; Introduced by $P$. Isgüven

${ }^{1}$ Division of Pediatric Endocrinology, SSK Göztepe Educational

Hospital, and Marmara University Sports Academy, Istanbul, Turkey

The shift in body composition towards fat is a problem in the management of diabetes, even in childhood. Objective: To investigate potential risks and clinical correlates of relatively increased body fat. Patients and Methods: We studied prepubertal, nonobese Type 1 diabetic children for the relationship of body fat mass parameters such as bioelectrical impedance analysis (BIA), body mass index (BMI), skinfold thickness (ST, biceps, triceps, subscapular, suprailiac, femoral and their sum), body circumference (upper arm, femoral, waist, hip, waist/hip) measurements, serum leptin and compared them with clinical parameters such as age, gender, height SDS, age at disease onset, diabetes duration, glycemic control, insulin dose, serum lipids and microalbuminuria (MA). Twenty-five patients with diabetes duration $>2.5$ yrs were included (age $8.6 \pm$ $1.5, \mathrm{M} / \mathrm{F} 14 / 11$, BMI $16.4 \pm 1.5 \mathrm{~kg} / \mathrm{m}^{2}$ ). Results: Sum of ST and leptin were significantly increased in diabetic girls than boys $(p=0.03$ and 0.02$)$. Leptin was significantly correlated with insulin dose and MA $(\mathrm{p}<0.001)$. MA was also correlated with sum of ST $(p<0.01)$, waist circumference $(p<0.02)$ and $\%$ fat mass in BIA $(p<0.05)$. Conclusions: Our findings supported the view that gender and insulin are important regulators of leptin even before puberty. Also we concluded that increased body fat is related with microalbuminuria for which the causality remains to be explained. 
P3-498 Diabetes and Glucose Metabolism

Exercise Stress Test and Left Ventricular Function in Adolescents and Young Adults with Insulin Dependent Diabetes mellitus (IDDM)

H. Tsoka-Gennata 1; V. Pailopoulos*1; A. Kafourou*1;

G. Papadopoulos*1;K. Kassiou*1

${ }^{1}$ Aghia Sophia Children's Hospital, Athens, Greece

Diabetic patients are at increased risk of cardiovascular disease. Since there is today a possibility of patient directed intervention it is important to know the age of the disease onset before the development of clinical symptoms and signs. Objective: To evaluate the response to the exercise stress as well as the cardiac function of our older patients and their relation to known risk factors for cardiovascular disease. Patients and Methods: Thirty IDDM patients, 17 boys and 13 girls, with a mean age of 16.7 years (range 10-25) have been studied. A symptom-limited exercise test has been performed and exercise time, work performed, maximum heart rate, maximum systolic and diastolic blood pressure and ST segment on electrocardiogram have been recorded. A Doppler echocardiography has also been done and left ventricular systolic and diastolic function has been assessed. All these parameters have been correlated with common risk factors for cardiovascular disease in diabetes: age, disease duration, smoking, BMI, HbAlc, dyslipidemia and microalbuminuria. Thirty normal subjects have served as controls for the heart tests. Results: Mean age at diagnosis of IDDM was 9.6 years $(2.7-15.3)$ and mean disease duration 7 years $(2.7-15.3)$. Mean BMI was 22.7 (16.8-12.9) and mean HbAlc of the past two years $8.86 \%$ (6.0-12.7). Seven patients had dyslipidemia. Four patients had elevated urinary microalbumin excretion and one had diabetic retinopathy. All patients had a normal exercise test but in four cases a slightly elevated systolic and diastolic pressure has been recorded and in another two cases a significantly high heart rate has been noted. One patient had a reduced diastolic function (E/A 1) on Doppler echocardiography. The above results did not differ from the results of the control group. In the study group there was a statistically significant correlation between DMI and shorting fraction $(\mathrm{p}=0.050)$, work performed $(p=0.009)$ and maximum diastolic pressure $(p=0.017)$. Conclusions: In this group of patients our study did not reveal any significant cardiovascular abnormality. Further studies are required in order to clarify the relation between risk factors and the age of onset of cardiovascular disease in IDDM patients.

P3-499 Diabetes and Glucose Metabolism

\section{Superoxide Dismutase in Peripheral Blood Mononuclear Cells of Type 1 Diabetic Children and Their Siblings}

J. Boiko ${ }^{1}$; M. Zafranskaya*2; E. Anisovetz*2

${ }^{1}$ Belarusian State Institute for Medical Studies, Minsk, Belarus;

${ }^{2}$ International Sakharov Environmental University, Minsk, Belarus

Free oxygen radicals play a key role in pathogenesis of type 1 diabetes. It is known that the condition of beta-cells antioxidant defense influences the degree of their destruction during the disease development. At the first stages the amplified oxidative stress takes place, that necessitates antioxidant therapy in these patients. Using the antioxidants in a regulation of cell destruction cycle was shown in vitro on immune cells and endotelium. Objective: To study the natural antioxidant enzyme, superoxide dismutase (SOD), concentration in peripheral blood mononuclear cells (PBMC) of young diabetics and their siblings, as a criterion of general antioxidative defense status of organism including beta-cells. Materials and Methods: PBMC of 18 diabetics $(13.8+4.0 \mathrm{yrs}$; mean age of diabetes: $4.5+1.3 \mathrm{yrs}$ ), 21 their non-diabetic siblings (mean age: $14.4+3.8 \mathrm{yrs}$ ) and 15 similar age healthy controls were allocated on a FicollVerografinum beta-1.077 gradient. SOD was determined in PBMC lysate (conc. $2 \times 10^{6} / \mathrm{ml}$ ) by indirect spectrophotometry method. Results: SOD inhibition in PBMC was $40.36+4.2 \%(140.4+11.8 \mathrm{U} / \mathrm{ml})$ in control group. No significant difference was observed in diabetic children and their siblings. However, in $44.4 \%$ of diabetics the SOD contents in PBMC was reduced more than on 1.5 sigma. In their healthy siblings the SOD concentrations were also lower than those in controls $(\mathrm{p}<0.05)$. All children were treated with antioxidants. After the course of therapy ( 2 months), a significant decrease of amount of diabetics and their siblings having early SOD in low concentration was found $(44.4 \%$ vs. $28.5 \%)$. Conclusion: Our data suggest that natural antioxidant defense is decreased in non-diabetic children with family history of type 1 diabetes and confirm its prevention by an antioxidant therapy.

\section{P3-500 Diabetes and Glucose Metabolism \\ Partial Remission Phase and Metabolic Control in Type 1 Diabetic Children and Adolescents \\ A. Buyukgebiz 1 ;E. Bober*1; B. Dundar*1 \\ ${ }^{1}$ Dokuz Eylül University, Izmir, Turkey}

The remission phase while residual beta cell function is still present in recently diagnosed type 1 diabetes is very important because of the potential for pharmacological intervention to preserve this function. Objective: To evaluate the natural course and characteristics of remission phase with metabolic control in children and adolescents with IDDM. Patients and Methods: A retrospective study was performed on patients diagnosed with IDDM under the age of 18 years during the years 1991-1998. Sixty-two patients whose medical reports were available were included into study. We collected data from hospital records at diagnosis and from following charts of the first two years after diagnosis. The duration of history of symptoms, history of infection, presence of diabetic ketoacidosis (DKA), hospitalization period, basal C-peptid levels, insulin doses at diagnosis and weight, insulin doses and $\mathrm{HbAlC}$ concentrations at each visits were recorded. Entrance time and duration of remission was calculated in months based on three monthly intervals of follow-up visits and occasional intermediate visits and phone calls in case of problems; hypoglycemia, infection etcWe defined partial remission as insulin requirement $0.5 \mathrm{U} /$ $\mathrm{kg} /$ day and total remission as $0.1 \mathrm{U} / \mathrm{kg} /$ day. Results: Thirty-five patients entered partial remission (56.5\%). We observed similar remission rates in those aged $<10$ and $\geq 10$ years at diagnosis and in boys and girls. History of infection and presence of DKA were associated with lower occurrence rate of remission $(\mathrm{p}=0.001$ and $\mathrm{p}=0.001)$ and observed more under the age of 10 years $(\mathrm{p}=$ 0.0001 and $p=0.0001)$. The average insulin requirement per $\mathrm{kg}$ body weight calculated at diagnosis decreased with increasing age $(\mathrm{r}=-0.31, \mathrm{p}=0.012)$. Entrance time to remission was about $1.36 \pm 1.03$ months after diagnosis and correlated with insulin requirement at discharge of hospital $(\mathrm{r}=0.63, \mathrm{p}=$ $0.000)$. Duration of remission was about $11.67 \pm 5.82$ months and much longer in boys than girls $(\mathrm{p}<0.05)$. Six patients entered total remission for $3.80 \pm$ 3.73 months and all of them were boys. Median HbA1C concentrations throughout the first year of disease was significantly lower in patients who entered remission $(7.31 \pm 1.24 \%$ vs. $8.24 \pm 1.47 \%, p<0.05)$. However this difference was not observed in the second year of disease. Conclusion: History of infection and presence of DKA were associated with young age and these were the most important factors with decreased remission rate in newly diagnosed IDDM patients. Patients in remission group showed better metabolic control with less exogenous insulin requirement during the remission period. 
P3-505 Diabetes and Glucose Metabolism

\section{Type 1 Diabetes and Celiac Disease in a Pediatric} Catalan Population

C. Farre ${ }^{* 1} ;$ M. Torres ${ }^{* 1} ;$ C. Pavia ${ }^{1} ;$ M. Hernandez ${ }^{* 1} ;$ V. Varea ${ }^{* 1}$; P. Vilar*1

${ }^{1}$ Hospital Sant Joan de Déu, Barcelona, Spain

Type 1 diabetes (DM1) and celiac disease (CD) are both immunologic disorders that could be present in genetically susceptible individuals. Their association is relatively frequent and different serological markers are used with the aim to detect silent CD in DM1 patients. Objective: 1) To study the frequency of silent CD in our paediatric DM1 population using serological markers previous intestinal biopsy. 2) To compare the reference qualitative and manual serological method against two differents quantitative and automated Elisa methods. Patients and Methods: In a cohort of 233 DM1 patients aged ranged from 0.9 to 18 years, we measured $\operatorname{IgA}$ (or IgG class in patients with IgA deficiency) antiendomysium antibodies (AEA) using monkey oesophagus as substrate of indirect immunofluorescence, that serves as reference method. Besides, we analyze IgA (or IgG) antigliadin antibodies (AGA, Pharmacia Co) in 121 of the 233 patients, and $\mathrm{IgA}$ (or IgG) tissue transglutaminase antibodies (Quanta Lite ${ }^{\mathrm{TM}} \mathrm{t}-\mathrm{TG}$, Inova Diagnostics Inc) in the remaining 112 cases. Antigliadin and tissue transglutaminase antibodies were analysed using a quantitative and automated Elisa method. Results: We found positive IgA (or IgG) AEA in 15 of the 233 DM1 patients $(6.4 \%, 15 / 233)$. Ten of them $(4.3 \%, 10 /$ 233) were diagnosed by $C D$ using intestinal biopsy. In two AEA positive patients, biopsy is not already performed. In the last three AEA positive patients, intestinal biopsy showed minimal abnormalities of the intestinal villous, these patients have to be controlled in order to detect CD activation. Whether we compare AGA and t-TG concentration with AEA results, we can see that AGA concentration is normal in five AEA positive DM1 patients and elevated in ten AEA negative. Moreover, t-TG ab is normal in two AEA positive DM1 patients and elevated in nine AEA negative. Six DM1 patients had IgA deficiency $(2.6 \%, 6 / 233)$ and one of them was diagnosed by celiac disease. Conclusions: The frequency of celiac disease in our diabetic population ranges $4.3 \%$ to $6.4 \%$. From our results it seems that t-TG not improve the accuracy and specificity of AGA, and non automated AEA is the best serological marker to detect silent or latent CD in DM1 patients.

\section{P3-506 Diabetes and Glucose Metabolism}

Day/Night Variations in the Ambulatory Blood Pressure Monitoring in Children and Adolescent with Type 1

Diabetes

R. Cardani*1;A. Salvatoni*1;R. Rovelli*1;E. Piantanida*1

C. Orsatti*1; L. Nespoli*1; Introduced by P. Brambilla

${ }^{1}$ Insubria University, Varese, Italy

A reduction in the day-night variations of blood pressure has been reported in IDDM patients as a sign of incipient hypertension. Objective: To compare the day-night variation of blood pressure in children and adolescents with different IDDM duration with that of healthy controls and to find correlations, if any, between blood pressure and insulin requirement, metabolic control, AER and cotecholamines in plasma and urines. Patients and Methods: We studied 28 subjects with IDDM and 16 healthy controls (12 boys and 4 girls) aged. The duration of IDDM was less than 5 years (Group 1) $(2.0 \pm 1.5$ years) in 14 subjects ( 9 boys and 5 girls aged $11.1 \pm 4.4$ ) and more than 5 years (Group 2) $(9.4 \pm 3.2)$ in 14 subjects ( 9 boys and 5 girls aged $17.5 \pm 5.7)$. All patients were in treatment with 2-4 daily subcutaneous injections of short- and intermediateacting human insulin. The metabolic control was assessed by daily monitoring of blood glucose and by measurement of HbA1c (DCA2000, Bayer) every two months. 24 hrs ambulatory blood pressure monitoring (ABPM) was measured by oscillometric and auscultatory technique using a TM2421 equipment (AND \& D Company Ldt, Tokio, Japan). Urinary and plasmatic noradrenaline, adrenaline and dopamine were measured by HPLC. Student's t test, ANOVA, simple, multiple and stepwise regression were used for statistical analysis. Results: Mean systolic and diastolic ABPM, adjusted for age, height and weight were similar in Group 1, Group 2 and Controls; however Group 2 showed a lower drop of diastolic BP during night-time compared to controls $(10 \pm 12 \%$ vs. $18 \pm 7 \% ; p<0.05)$. Direct significant correlations were found between $\mathrm{HbA} 1 \mathrm{c}$ and mean nocturnal diastolic blood pressure $(\mathrm{p}>0.05)$, insulin requirement and plasmatic adrenaline, noradrenaline and dopamine $(\mathrm{p}<0.5)$ and mean nocturnal/diurnal diastolic blood pressure ratio and urinary dopamine. Conclusion: Our results confirm the observation that patients with IDDM for more than 5 years have a reduction in the day-night variation of blood pressure and suggest that an increase in dopaminergic system activity may be responsible for this condition.

P3-507 Diabetes and Glucose Metabolism

Cross-Sectional and Longitudinal Evaluation of Fat and Bone Mass in IDDM Patients by DXA

R. Rovelli*1; A. Salvatoni*1; M. Broggini*2;C. Orsatti*1;

S. Salvatore ${ }^{* 1} ;$ R. Cardani*1; L. Nespoli*1; Introduced by P. Brambilla

${ }^{1}$ Insubria Universityy, Varese, Italy; ${ }^{2}$ Ospedale di Circolo, Varese, Italy

Increase in fatness and bone mineral loss has been reported in IDDM patients. Objective: To answer the following questions: Which is the impact of IDDM on fat and bone mass?, are fat and bone mass of diabetic children and adolescents different from those of their healthy mates?, which is the influence of insulin treatment and metabolic control on fat and bone mass? Patients and Methods: We studied 42 children and adolescents (13 girls and 29 boys) with IDDM. The duration of the disease was $14.6 \pm 5.1$ years. 24 of them were also studied at the onset of the disease. We collect for each patients the following parameters: height, weight, BMI, pubertal stage, insulin requirement and HbAlc. Fat mass (FM) and bone mineral content (BMC) were evaluated by DXA using a DPX Lunar equipment. The patients were compared with 76 healthy controls matched for age and sex. The sample size was calculated in order to achieve a power of $>0.90$ in detecting at least $10 \%$ variation in BMC (estimated SD $179 \mathrm{~g}$ ), and 5\% variation in fat mass (estimated SD 7.07\%). Therefore we recruited at least 16 patients for the longitudinal study. Multiple regression analysis, ANOVA, paired Student's t-test and simple regression were used for statistical analysis. Results: BMC was adjusted for weight and FM for age and gender. After adjustment BMC and FM values were similar in patients and controls and within the group of IDDM patients at the onset of the disease and thereafter. We found a statistically significant correlation $(\mathrm{p}<0.05)$ between BMC adjusted for weight and duration of IDDM only in boys. Insulin requirement and $\mathrm{HbA1} \mathrm{c}$ were not correlated with BMC and FM. Conclusion: IDDM does not influence FM and BMC in children and adolescents. A reduction in BMC in male patients with IDDM of long duration must be confirmed.

\begin{tabular}{llll}
\hline & IDDM (onset) & IDDM (thereafter) & Controls \\
\hline & $\mathrm{n}=24$ & $\mathrm{n}=42$ & $\mathrm{n}=76$ \\
$\mathrm{BMC}^{\wedge}$ & $1401 \pm 198$ & $1392 \pm 201$ & $1372 \pm 180$ \\
$\% \mathrm{FM}^{\S}$ & $19.4 \pm 10.9$ & $18.1 \pm 7.0$ & $20.1 \pm 6.9$ \\
\hline Longitudinal study $(n=16)$ & & \\
$\mathrm{BMC}^{\wedge}$ & $1429 \pm 222$ & $1414 \pm 197$ & $\mathrm{t}=0.307 ; \mathrm{p}>0.05$ \\
$\% \mathrm{FM}^{\S}$ & $19.6 \pm 11.0$ & $17.8 \pm 7.4$ & $\mathrm{t}=0.756 ; \mathrm{p}>0.05$
\end{tabular}

$\wedge$ Adjusted for weight; $\S$ adjusted fot age and gender. 
P3-508 Diabetes and Glucose Metabolism

HLA-DQ2 in Catalan Type 1 Diabetes Population

C. Farre*1; T. Marques*1; M. Hernandez*1; C. Pavia ${ }^{1} ;$ M. Torres ${ }^{* 1}$;

P. Vilar*1; V. Varea*1

${ }^{1}$ Hospital Sant Joan De Déu, Barcelona, Spain

The likely explanation for the frequent simultaneous occurrence of celiac disease (CD) in Type I diabetes (DM-I) is that both of them have a similar genetic background. Class-II HLA genes encode cell-surface molecules which bind and present antigenic peptides to $\mathrm{T}$ cells and have a major role in the pathogenesis of many autoimmune disorders. It is known that DQA $1 * 0501$ and DQB $1 * 0201$ alleles (HLA-DQ2 haplotype) are primarily related to CD. Objective: To study the frequency of DQ2 haplotype in a sample of Catalan Type 1 diabetes population. Patients and Methods: We studied eight DM1 patients with positive antiendomysium antibodies (AEA). CD was confirmed by intestinal biopsy. Thirty-five DM1 patients with negative AEA, sixty CD patients and sixty-four ethnically matched controls from the general population. DQ2 haplotype was determined by SSCP-PCR. Whole genomic DNA was isolated by phenol extraction and ethanol precipitation, Gene Amp PCR2400 system (Perkin Elmer) was used for amplification and PCR products were electrophoresed on a $1.5 \%$ agarose gel. Examination under UV light confirmed amplification efficiency. This method detects the presence or absence of at least one copy of DQA $1 * 0501$ and DQB $1 * 0201$. Results: Five of the eight AEA positive diabetic patients were diagnosed of celiac disease by intestinal biopsy. The three remaining, DQ2 positive, showed minimal non-specific abnormalities of the intestinal villous.

Frequency of DQ2 haplotype in DM1/AEA+, DM1, CD and Controls

\begin{tabular}{lllll}
\hline & DM1/AEA+ & DM1 & CD & Controls \\
\hline DQA1*0501 & $87 \%(7 / 8)$ & $77 \%(27 / 35)$ & $95 \%(57 / 60)$ & $48 \%(31 / 64)$ \\
DQB1*0201 & $75 \%(6 / 8)$ & $77 \%(27 / 35)$ & $98 \%(59 / 60)$ & $40 \%(26 / 64)$ \\
DQ2 & $75 \%(6 / 8)$ & $68 \%(24 / 35)$ & $93 \%(56 / 60)$ & $25 \%(16 / 64)$ \\
\hline
\end{tabular}

Conclusions: We suggest that the three diabetic patients with positive antiendomysium antibodies, positive DQ2 and minimal abnormalities in the jejunal biopsy, have latent $\mathrm{CD}$. The frequency of DQ2 haplotype obtained in our DM1 population confirms the higher genetic susceptibility to $\mathrm{CD}$ in these patients.

P3-509 Diabetes and Glucose Metabolism

\section{Occurrence of Coeliac Disease after the Onset of Insulin-Dependent Diabetes mellitus: A Six Years Follow-Up}

R. Bonfanti*1; G. Barera ${ }^{2}$; M. Viscardi ${ }^{2}$;E. Bognetti ${ }^{2}$;F. Meschi' ;

C. Bianchi ${ }^{2}$; G. Chiumello2; Introduced by C. Giuseppe

${ }^{1}$ Istituto Scientifico H San Raffele, Milano, Italy; ${ }^{2}$ Italy

The prevalence of coeliac disease (CD) as determined by screening programs among children and adolescents with insulin-dependent diabetes (IDDM) is higher than in general population, with rates of $1.1-7.8 \%$. These figures are probably underestimated, since many patients with positive antibody tests don't agree to small-bowel biopsy. Only occasionally CD precedes the onset of IDDM; more often it is diagnosed at IDDM onset or shortly after. Few studies focused on the occurrence of CD at the onset of IDDM and after the clinical onset by long-term longitudinal study. The aim of this study was to evaluate the occurrence of CD at the clinical onset of IDDN and the development of new cases of CD during a six years follow-up. We studied 275 patients $(116 \mathrm{~F}$, $159 \mathrm{M}$; mean age $8.4 \mathrm{yrs}$; range $0.3-26.7 \mathrm{yrs}$ ) at the onset of IDDM. One patient was on gluten free diet because a previous diagnosis of $\mathrm{CD}$. A longitudinal prospective evaluation was performed in $223 \mathrm{pts}$ at $1 \mathrm{yr}, 170 \mathrm{pts}$ at $2 \mathrm{yrs}, 117$ pts at 3,73 pts at 4,44 pts at 5,22 pts at 6 yrs from onset of disease. IgAantiendomysium antibodies (AEA) test on monkey's oesophagus was performed at onset and at each year of follow-up. Serum IgA vere also determined, IgG AEA and IgG antigliadine antibodies were tested in patients with IgA deficiency $(<0.05 \mathrm{~g} / \mathrm{l})$. Results: At onset were found 14 AEA positives with 7 biop- sies all athrophy; At 1 year 4 AEA+, 2 biopsied, at 2 yrs 6 AEA+ with 4 biopsies, at 3 yrs 7 AEA+ with 4 biopsies, at 4 yrs 4 AEA+ with 3 biopsies, no more positives at 5 and 6 yrs follow-up. Two patients were IgA deficient but IgG test negative. Two patients with CD diagnosis at follow-up were already positive at onset. Conclusion: Our data confirm an increased prevalence of CD in IDDM population $(6.2 \%)$ with an annual incidence of $1.38 \%$ (respectively $0.9 \%, 1.2 \%, 2.56 \%, 2.74 \%, 0 \%$ and $0 \%)$. A small group of patients $(11 / 274)$ had a variable week AEA positivity that changed during time. Our study suggests the opportunity to screen IDDM patients for CD with AEA, at the onset and also during years near onset. Same patients show fluctuation of AEA levels as expression of $\mathrm{CD}$ latency.

P3-510 Diabetes and Glucose Metabolism

\section{Bile Formation and Transporter Proteins in}

Streptozotocin-Induced Diabetic Rats

W. van Waarde ${ }^{* 1} ;$ H.J. Verkade ${ }^{* 1} ;$ H. Wolters ${ }^{* 1} ;$ R. Havinga*1

M. Müller ${ }^{* 2} ;$ P.J. Sauer ${ }^{* 1} ;$ F. Kuipers ${ }^{* 1}$; Introduced by R.J. Odink

${ }^{1}$ Beatrix Children's Hospital, Groningen, ${ }^{2}$ Groningen University

Hospital, Groningen, The Netherlands

Diabetes mellitus is associated with changes in bile formation, such as increased biliary secretion of bile salts and cholesterol. As cholesterol is excreted from the body almost exclusively via bile, either as free compound or after conversion to bile salts, bile formation is important for cholesterol homeostasis. Underlying mechanisms for the changes in bile formation in diabetes are unknown. Several ATP-Binding Cassette (ABC) transporter proteins at the bile canalicular membrane are involved in hepatobiliary transport of bile constituents. Objective: We studied the effects of streptozotocin-induced diabetes (STZ) on hepatic expression of specific transporter proteins and on related changes in bile formation in rats. Methods: In control, STZ and insulintreated diabetic (STZ-INS) rats, bile formation was studied under basal conditions and during stepwise increasing i.v. doses of taurocholate to determine bile salt secretory rate maximum (SRm). Hepatic messenger RNA (mRNA) and protein levels of transporters were studied by reverse-transcription polymerase chain reaction, immunoblotting and immunohistochemistry. Results: In STZrats, basal biliary secretion rates of bile salts $(+260 \%)$, cholesterol $(+25 \%)$ and phospholipids $(+520 \%)$ were increased, compared to controls, while glutathione secretion was reduced $(-97 \%)$. The SRm of taurocholate in STZ-rats was increased compared to controls $(+130 \%)$. Expression of the multidrug resistance P-glycoprotein type $2(\mathrm{mdr} 2)$ transporter, essential for phospholipid secretion, was increased at mRNA $(+100 \%)$ and protein $(+500 \%)$ level in STZ rats, while protein levels of the canalicular bile salt export pump (bsep) were unchanged. Protein levels of the multidrug resistance associated protein 2 $(\mathrm{mrp} 2)$, involved in glutathione transport, were decreased $(-55 \%)$ in STZ-rats compared to controls. Insulin treatment partially reversed the effects of STZ on bile formation and transporter expression. Conclusions: Streptozotocin-diabetes is associated with changes in expression of specific ABC transporter proteins and altered bile formation in rats. Increased mdr2expression, leading to phospholipid hypersecretion may protect against bile salt-induced cholestasis and, thereby, lead to higher taurocholate SRm in STZ-induced diabetic rats. 
P3-511 Diabetes and Glucose Metabolism

\section{Type 1 Diabetic Children: Long Term Follow-Up by Fluorescein Angiography Beginning in the First Months of Disease}

S. Salardi ${ }^{1}$;F. Rubbi ${ }^{2}$; R Puglioli*2; S. Zucchini ${ }^{1}$; L. Ragni*1;

A. Brancaleoni*2; L. Bacchi-Reggiani*3; C. Fabiano*1; E. Cacciari ${ }^{1}$

${ }^{1}$ Department of Pediatrics, ${ }^{2}$ 2nd Oculistic Clinic, ${ }^{3}$ Department of

Cardiology, University of Bologna, Bologna, Italy

Little is known about minimal retinal lesions occuring in the first months of disease in diabetic children. Objective: To detect any early retinal change and to evaluate its progression in children diagnosed of type 1 diabetes mellitus at our Pediatric Clinic. Patients and Methods: From 1979 to 1997 we examined at diagnosis or within 15 months from the diabetes onset by fluorescein angiography 130 young diabetic patients (mean age at diagnosis $10.08 \pm 2.62$ yrs). In 112 cases a patient follow-up by fluorescein angiography was performed every 1.26 years with a mean of 5.41 fluorescein angiographies/patient. The stage of retinopathy was graded to detect minimal lesions. Results: At first examination 14 out of $127(11 \%)$ readable angiographies showed minimal retinal changes. There was no statistically significant difference between the patients with or without lesions for all the parameters considered. The 112 patients examined during follow-up were divided as follows. Group A: No retinopathy at first examination; Group A1: no retinopathy during follow-up; Group A2: retinal changes during follow-up; Group B: retinal changes from the first examination. Mean HbA1 value evaluated during the whole follow-up was lower in Group A1 than group A2. HbA1 levels at onset of the disease was significantly different in the 3 groups: in Group A1 it was lower than in Group A2 and in Group B. Conclusions: The presence of early lesions in the first year of disease in $11 \%$ is probably due to the method of examination, which may detect even minimal retinal changes. This may be correlated with the acute metabolic failure present at onset of disease. The prolonged follow-up seems to demonstrate that the early changes are not necessarily a negative prognostic factor in the evolution of diabetic retinopathy. We confirm that duration of diabetes and metabolic control are the main factors influencing the course of retinopathy in these young patients.

P3-512 Diabetes and Glucose Metabolism

Early Signs of Polyneuropathy in Adolescents with Type 1 Diabetes

P. Riihimaa* ${ }^{* 1} ; K$. Suominen ${ }^{2} ; U$ Tolonen ${ }^{* 2} ; M K_{\text {nip }}{ }^{3} ; P$ Tapanainen ${ }^{4}$ ${ }^{1}$ University of Oulu, Oulu, Finland; ${ }^{2}$ Department of

Neurophysiology, University of Oulu, Oulu, Finland; ${ }^{3}$ Department of Pediatrics, University of Tampere, Tampere, Finland; ${ }^{4}$ Department

of Pediatrics, University of Oulu, Oulu, Finland

Impact of peripheral nerve conduction velocity (NCV) and autonomic nerve system (ANS) in adolescent patients with type 1 diabetes was studied. Objective: To evaluate the peripheral neurological function in adolescents with type 1 diabetes (age 9-19 yr, diabetes duration over 2 yr). Patients and Methods: One hundred adolescent patients (21 in pubertal stage T I; 60 in T II-IV; 19 in $\mathrm{T} \mathrm{V}$ ) and 100 healthy age and sex matched controls (15 in T I; 56 in T II-III; 29 in $\mathrm{T} \mathrm{V}$ ) participated in the study. The motor and sensory NCV in right median nerve and peroneal and sural nerves was measured bilaterally and adjusted for the mean height and skin temperature. ANS was evaluated with heart rate variability in rest, deep breath and during active standing. Distal polyneuropathy was defined as NCV 5th percentile of the healthy controls in three or more nerves. The mean duration of diabetes was $7.0 \mathrm{yr}$ and the mean HbAlc was $8.5 \%$. Results: Eleven patients $(5 \mathrm{~F} / 6 \mathrm{M})$ had distal polyneuropathy. Three of these 11 patients were prepubertal, three in T II-IV and five in T V. NCV in the median, peroneal and sural nerves were significantly lower in the whole study group of the patients than in the healthy controls. There were no significant differencies in heart rate in rest, in DB or during active standing between the patients and the controls. The patients with $\mathrm{HbAlc}$ over $8.5 \%$ had significantly slower peroneal NCV bilaterally than the patients with HbA1c $8.5 \%$ or below. No such difference was observed between the patients with disease duration over $7.0 \mathrm{yr}$ and below $7.0 \mathrm{yr}$. Conclusion: Adolescent patients with type 1 diabetes had obvious signs of mixed sensorimotor polyneuropathy. No clear difference in ANS function tests were observed between the patients and the controls. This implies that ANS may be more resistant to diabetic neuropathy or factors other than those involved in the development of sensorimotor neuropathy may contribute to autonomic neuropathy.

\section{P3-513 Diabetes and Glucose Metabolism \\ Two Mutations on Exon 9 of the Glucokinase Gene Associated with Hyperinsulinemia}

L. Guazzarotti*1;I. Testa*2; P. Fumelli*3; C. Bellanne-Chantelot*4; E. Bartolotta* ${ }^{*} ;$ Introduced by $R$. Lorini

${ }^{1} \mathrm{~S}$. Lucia Hospital, Recanati, Italy; ${ }^{2}$ University of Ancona, Ancona, Italy; ${ }^{3}$ Department of Diabetology-INRCA, Ancona, Italy; ${ }^{4}$ Fondation Jean Dausset-CEPH, Paris, France

Maturity Onset Diabetes of the Young (MODY) is normally associated with early, mild or severe, impaired insulin secretion and late insulin resistance. However, a subtype of MODY associated with insulin resistance has been reported, but no mutations of known MODY genes have been found in the affected patients. Moreover, a mutation on exon 10 of the glucokinase (GCK) gene associated with familial hyperinsulinemia has been described. We studied MODY2 and MODY3 gene mutations in two children (1 pubertal and 1 prepubertal) with impaired fasting glucose associated with hyperinsulinemia (studied with oral and i.v. glucose load) and family history of NIDDM or late-onset IDDM. Two missense mutations on exon 9 of the GCK gene were found in both subjects. One of these patients was a 16 year old female, with normal body weight, who suffered from hypoglycemia during childhood. Her father, who showed the same mutation, had an impaired fasting glucose not associated with clinical symptoms of hyperinsulinemia; and the grandfather, who was not genotyped, had NIDDM. The gene mutation was an aminoacid substitution at the codon 404 (I404S). This mutation was also found in another of our MODY families, but in this case it was not associated with hyperinsulinemia. The second patient was a 5 year old boy, with normal body weight, who showed very high values of plasma insulin for his age (FPIR after IVGTT $=299 \mu \mathrm{U} / \mathrm{ml}$ ). The father, who developed insulin-dependent diabetes at the age of 27, did not show the mutation. Therefore the mutation of the proband, a novel aminoacid substitution very close to the first one, at the codon 407 (G407S), was a de novo mutation. No diabetes was present in the third generation of this family, so the father probably has a type 1 diabetes (HLA-DR3 + and GAD/IA2 +/-) unrelated to the hyperglycemia of the son. The only other mutation found until now in the GCK gene, related to hyperinsulinemia, was an aminoacid substitution at the codon $455(\mathrm{~V} 455 \mathrm{M})$. This mutation, when expressed in vitro, was shown to increase the affinity of glucokinase for glucose, resulting in a higher rate of glycolysis at low glucose concentrations and therefore in a higher rate of insulin secretion. The mutations described here, located in the same GCK gene region, could have a similar activating role on the glucokinase enzyme. The different phenotype associated with the I404S mutation could represent a variable penetrance. A secondary $\beta$-cell failure should be the long-term effect of these mutations, leading to overt diabetes. 
P3-514 Diabetes and Glucose Metabolism

Genotype May Not Predict Phenotype in Pedigrees Carriyng the R272H Mutation of the HNF-1 $\alpha$ Gene

L. Guazzarotti*1; F. Panicari*2; C. Sgattoni*1; G. Mariani*1;

R. Pecora*1;E. Bartolotta*1; Introduced by R. Lorini

${ }^{1}$ S. Lucia Hospital, Recanati, Italy; ${ }^{2}$ Laboratory of Clinical Pathology,

Recanati, Italy

It is known that the severity and age of diagnosis of diabetes varies widely within families with genetic diagnosis of Maturity Onset Diabetes of the Young (MODY). We describe two families carrying a missense mutation at the codon 272 of the HNF-1 $\alpha$ gene responsible for MODY type 3, where the variety of the phenotype was so extensive that the clinical predictive value of the genetic testing was impaired. One of the families was a large pedigree, of which $16 \mathrm{mem}$ bers were genotyped. A weak correlation between phenotype and genotype was found. In fact, a young member of the pedigree, a 22 year old man, already developed overt NIDDM, while his sister, 8 years older and with a previous pregnancy, showed only an impaired fasting glucose. Another young member of the family a 24 year old man, had NIDDM, while two of his siblings, aged 17 and 20 years and carrying the mutation, had a normal fasting and post-oral load glucose. In contrast, one adult member of the same family, 47 years old and exhibiting an impaired glucose tolerance (IGT), was not a carrier of the mutation. In the second family we studied two brothers who showed the same mutation: the younger developed an insulin-dependent diabetes at the age of 14, and the older, showed only an IGT at the age of 37. The father developed NIDDM at the age of 50. HLA antigens and $\beta$-cell antibodies (GAD, IA2, IAA) were studied in the IDDM sibling. He showed the DR3 HLA antigen, which is related to type 1 diabetes, and anti-insulin antibodies and a very low titer of antiIA2 antibodies were also present. Factors affecting this phenotype variation would most likely not be environmental since it is observed in siblings. In conclusion, although genetic predictive testing is now possible for the majority of MODY families and is of clinical benefit, caution must be taken in pediatric and adolescence ages regarding the prediction of the clinical evolution of this metabolic disorder.
P3-516 Diabetes and Glucose Metabolism

Uncommon Early-Onset Acute Painful Neuropathy in a Boy with Type 1 Diabetes mellitus

M.V.B. Pescador*1; L.C. Fortes ${ }^{* 1} ;$ I.V. Brandi*2; M.C. Geiger*3, L. De Lacerda*3; W.O. Arruda*2;M.C.S. Boguszewski3 ; Introduced by M.C.S. Boguszewski

${ }^{1}$ Curitiba, Brazil; ${ }^{2}$ Neuromuscular Diseases Unit, Curitiba, Brazil;

${ }^{3}$ Pediatric Endocrinology Unit, Curitiba, Brazil

An acute neuropathy rarely occurs early in the course of insulin-dependent diabetes mellitus (IDDM). A 14-year-old boy was admitted at the Intensive Care Unit with diabetic ketoacidosis (DKA) when the diagnosis of IDDM was made. He reported a profound weight loss (approximately $10 \mathrm{~kg}$ ) during the months preceding onset. He developed an acute painful symmetrical distal neuropathy some days after his admission. He presented distal muscle weakness and he could not walk. Reflexes were diminished or absent in ankle and knee. Nerve conduction study showed absence of motor and sensory action potentials in both legs. Needle electromyography evaluation showed signs of denervation in distal muscles of the lower limbs. The patient was treated according to the routine for DKA and received carbamazepine to alleviate the painful paresthesis. A good glycemic control and weight gain occurred after beginning treatment with insulin. Six months after the diagnosis, the patient was pain free and paresthesia in feet remained as the only symptom. Comments: Acute painful neuropathy is a rare condition that may affect young diabetic patients at the start or shortly after development of the disease. Although a nerve biopsy was not performed, we can suggest that acute disequilibrium of the metabolic state and/or the autoimmune process may facilitate the occurrence of neuropathy in these patients.
P3-515 Diabetes and Glucose Metabolism

\section{Defective Function of Fas in Patients with Type I (Insulin-Dependent) Diabetes mellitus Associated with Other Autoimmune Diseases}

S. De Franco*1; S. Bonissoni*1; A. Petri*1; M. Bragardo*1,

F. Cadario ${ }^{* 1}$;A. Aguzzi*1;F. Sacco*1;F. Cerutti*2;A. Corrias ${ }^{* 2}$.

U. Dianzani*1; G. Bona ${ }^{1}$; Introduced by G. Bona

${ }^{1}$ University of Eastern Piedmont, Novara, Italy; ${ }^{2}$ Regina Margherita

Hospital, Turin, Italy

Aims: Fas (CD 95) triggers programmed cell death. It is also involved in cellmediated cytotoxicity and in shutting off the immune response. Inherited lossof-function mutations of its gene or downstream alterations of its signaling pathway cause the immune/lymphoproliferative syndrome (ALPS). This study evalutes Fas function in patients with diabetes associated with other autoimmune diseases (autoimmune polyreactive patients). Patients and Methods: Cell death induced by anti-Fas monoclonal antibody was investigated in T cells from three group of patients with Type I diabetes mellitus (IDDM T-1): IDDM T-1 only $(n=13)$, IDDM T-1 plus thyroiditis $(n=13)$ and IDDM T-1 plus other autoimmune diseases $(n=6)$, and 19 patients with thyroiditis alone. The study was extended to parents of four patients with IDDM T1 plus thyroiditis. Results: Frequency of resistence to Fas-induced cell death was significantly higher in patients with IDDM T-1 plus thyroiditis (77\%) and plus other autoimmune diseases $(67 \%)$ than in normal controls $(3 \%)$; and in patients with IDDM T-1 plus thyroiditis than in thyroiditis alone (16\%) and IDDM T-1 alone $(23 \%)$. Fas was always expressed at normal levels and no mutations were detected in one IDDM T-1 plus thyroiditis and in one IDDM T-1 plus autoimmunitis Fas-resistant patient. The defect was likely to due to genetic component since parents were Fas- resistant even if healthy. Conclusion: These data suggest that Fas defects may be a genetic factor involved in the development of other autoimmune diseases in patients with IDDM T-1.

\section{P3-517 Diabetes and Glucose Metabolism}

IPF-1 Gene Mutations as a New Possible Cause of Neonatal Diabetes mellitus: A Case Report

A. Maret ${ }^{1}$; V. Schwitzgebel*2; B. Ritz-Laser*2;D. Balmer*3; J. Philippe *2; G. Theintz ${ }^{1}$

1 University Hospital of Lausanne, Lausanne, Switzerland;

2 University of Geneva, Geneva, Switzerland; ${ }^{3}$ University of Zürich,

Zürich, Switzerland

Introduction: Neonatal diabetes mellitus (NDM) is a very rare condition of variable outcome (transient vs permanent forms). Whereas the origin of most cases is still obscure, some are part of complex syndromes (Pearson, WolcottRallison) or associated with paternal uniparental disomy of chromosome 6 , particularly the transient form. We describe a case with permanent NDM and pancreatic exocrine insufficiency with preliminary results suggesting a novel mutation in the IPF-1 gene. Case Report: This term female baby was born with intra-uterine growth retardation $(2,140 \mathrm{~g} / 44 \mathrm{~cm})$ after a pregnancy marked by slight gestational diabetes. She was admitted at 12 days of age for poor weight gain despite marked appetite, followed by steatorrhea. Investigations: glycaemia: $43.7 \mathrm{mmol} / \mathrm{l}$; pH: 7.46; $\mathrm{BE}$ : $+0.8 \mathrm{mmol} / \mathrm{l}$; amylase: $4 \mathrm{U} / \mathrm{l}$; lipase: $15 \mathrm{U} / \mathrm{l}$; stool elastase: $13 \mu \mathrm{g} / \mathrm{l}$ (normal: > 200); ICA (baby + parents): negative; CT-scan: marked isolated pancreatic hypoplasia; genetic studies: suggest two point mutations in the IPF-1 gene (compound heterozygocity). Chromosome 6: normal biparental inheritance. Outcome: continuous subcutaneous insulin infusion and enzyme replacement therapy are required at 21 months of age; normal developmental milestones. Discussion: Endocrine and exocrine pancreatic failure suggest abnormal pancreatic development as confirmed by CTscan imaging. The homeodomain protein IPF-1 is critical for pancreas development: in transgenic mice, knock-out studies resulted in pancreatic agenesis. A point deletion of the IPF-1 gene in a patient with pancreatic agenesis has been identified recently (Stoffers et al, Nat Genet 1997;15:106). In the absence of clinical markers to differentiate transient from permanent NDM forms, genetic investigations represent a use-ful tool: there is a strong association between the transient NDM and paternal uniparental disomy for chromosome 6. On the other hand, IPF-1 gene anoma-lies point to permanent NDM with pancreatic hypoplasia/aplasia. 
P3-518 Diabetes and Glucose Metabolism

\section{Occurence of Microangiopathy after Long-Term Follow-Up (12-18 Years) in 40 Patients with Type 1 Diabetes of Childhood Onset \\ S. Rouleau*1; B. Bouhanick*1; H. Raguin*1; R. Coutant*1. \\ V. Rohmer*1; J.M. Limal \\ 1 University Hospital, Angers, France}

Objectives: To estimate the outcome of patients with type 1 (immune mediated) diabetes followed in one university hospital during at least 12 years. Methods: Retrospective study of medical records of 40 patients (24 F, 16 M) with type 1 diabetes from 1979 to 1997 . Results: Mean age at diabetes onset was $8 \pm 4$ years (1-16 years), and follow-up lasted $16 \pm 4$ years. Ketoacidosis revealed diabetes in $42 \%$ of the children. Mean HbAlc was $8.5 \pm 1.3 \%$ during the whole study time. Mean HbAlc was less than $7.5 \%$ in $17 \%$ of the patients, $7.5-8.5 \%$ in $38 \%$, and over $8.5 \%$ in $45 \%$. After 5,10 , and 15 years, $15 \%, 50 \%$, and $81 \%$ of the patients received multiple daily injections (2/day), respectively. The mean age at onset of puberty (Tanner stage II) was $12 \pm 1$ years in girls and $13 \pm 1$ years in boys. Mean HbAlc was $7.9 \pm 1.2 \%$ during the year before puberty onset, and $8.7 \pm 1.1 \%$ in the following 3 years, corresponding to a $10 \%$ pubertal increase of $\mathrm{HbAlc}$. Mild nonproliferative retinopathy was seen in $57.5 \%$ of the patients, at a mean age of $21 \pm 4$ years, after $13 \pm 5$ years diabetes duration. Proliferative retinopathy was seen in $10 \%$ of the patients, at a mean age of $25 \pm 2$ years, after $20 \pm 6$ years diabetes duration. No retinopathy appeared before the age of 15 years. Mean HbAlc was $9.6 \pm 1.8 \%$ in patients with proliferative retinopathy, $8.4 \pm 1.2 \%$ in patients with nonproliferative retinopathy, and $8.2 \pm 1.5 \%$ in those with no retinopathy $(\mathrm{p}<0.05$ proliferative vs. no retinopathy, $\mathrm{p}>0.05$ non proliferative vs no retinopathy). Persistent microalbuminuria (Albumin excretion rate $20 \mathrm{mg} / \mathrm{l}$ on 3 different urine collections) was diagnosed in $30 \%$ of the patients, at a mean age of $21 \pm 3$ years, after $11 \pm 4$ years diabetes duration. Mean HbAlc was $9.2 \pm 1.2 \%$ in patients with persistent microalbuminuria versus $8.2 \pm 1.3 \%$ in those with normal AER ( $p=$ 0.002 ). No patient with persistent microalbuminuria had mean HbAlc below $7.5 \%$. Twenty-five percent of the patients had both retinopathy and nephropathy. Conclusions: Glycemic control was similar in patients with non proliferative retinopathy and those with no retinopathy. Whereas proliferative retinopathy and nephropathy were both related to the level of glycemic control, we observed a trend for nephropathy to be more prevalent, occur earlier for lower HbAlc than proliferative retinopathy.

P3-519 Diabetes and Glucose Metabolism

Glucose Tolerance and Insulin Secretion in Children with IUGR before and during Two-Year Treatment with Growth Hormone

C.G. Hadjiathanasiou ${ }^{1}$; C. Theodoridis ${ }^{1} ;$ A. Papathanasiou ${ }^{1}$; C. Kyrou*1;A. Fotinou ${ }^{* 1} ;$ M. Foustoukou*1

${ }^{1}$ Department of Endocrinology, Kyriakou Childrens Hospital, Athens, Greece

Growth hormone (rhGH) has been used in recent years in clinical trials for the treatment of short children born small for gestational age. IUGR, however, is associated with increased risk of development of insulin resistance, hyperglycemia and non-insulin dependent diabetes mellitus in adult life. We wished to investigate whether this risk might be increased by rhGH treatment because of its potential detrimental effects on glucose metabolism. We studied prospectively 10 prepubertal children ( 6 boys, 4 girls) with IUGR (birth length and/or weight $<-2$ SD for gestational age), aged 2.2-9.2 years (mean chronological age $6.0 \pm 2.8$ years) during a 2-year period of rhGH therapy. All children had a baseline height $<3$ rd percentile, normal body weight, normal GH secretion in at least one provocative test and normal insulin like growth factor I levels. None of the children had a history of congenital infection, chromosomal abnormality, chronic disease or diabetes mellitus. GH was given at a dose of $1.4 \mathrm{IU} / \mathrm{kg} / \mathrm{wk}$ $(0.47 \mathrm{mg} / \mathrm{kg} / \mathrm{wk})$, in daily SC injections. Oral glucose tolerance test (OGTT) and $\mathrm{HbAlc}$ measurement were performed at the initiation and at the end of the 1st end 2 nd year of GH therapy (table).
OGTT and HbA1c in children with IUGR before and during rhGH therapy

\begin{tabular}{|c|c|c|c|c|c|c|c|c|c|}
\hline & \multicolumn{3}{|c|}{ Glucose (mg/dl) } & \multicolumn{3}{|c|}{ Insulin $(\mu \mathrm{U} / \mathrm{ml})$} & \multicolumn{3}{|c|}{ Hb Alc (\%) } \\
\hline & $0 \mathrm{~m}$ & $12 \mathrm{~m}$ & $24 \mathrm{~m}$ & $0 \mathrm{~m}$ & $12 \mathrm{~m}$ & $24 \mathrm{~m}$ & $0 \mathrm{~m}$ & $12 \mathrm{~m}$ & $24 \mathrm{~m}$ \\
\hline \multirow[t]{2}{*}{$0^{\prime}$} & 83 & 91.4 & 91.8 & 6.1 & 13.6 & 10.3 & 5.1 & 5.4 & 5.3 \\
\hline & \pm 6.4 & \pm 7.3 & $\pm 4.4^{*}$ & \pm 2.1 & $\pm 13.4^{*}$ & $\pm 3.0^{*}$ & \pm 0.6 & \pm 0.5 & \pm 0.7 \\
\hline \multirow[t]{2}{*}{$120^{\prime}$} & 114.9 & 117 & 113 & 20.9 & 41.2 & 34.2 & & & \\
\hline & \pm 27.2 & \pm 30.1 & \pm 10.8 & \pm 14.6 & $\pm 36.8^{*}$ & $\pm 18.2^{*}$ & & & \\
\hline
\end{tabular}

$* \mathrm{p}<0.05$.

Administration of rhGH for a period of 2 years in children with IUGR slightly increased fasting glucose levels, which however in every case remained within normal range. GH treatment did not alter either the 2-hour post OGTT glucose levels nor did it affect HbAlc. However, a significant increase of both fasting and 2-hour post OGTT insulin levels was observed at the 1st and 2nd year, suggestive of increased resistance to insulin action. Whether continued treatment might lead to eventual exhaustion of b-cells and development of diabetes mellitus remains to be clarified.

P3-520 Diabetes and Glucose Metabolism

Increased Insulin Sensitivity following Anticholinergic Therapy in Adolescent IDDM Girls

M. Halldin ${ }^{* 1} ;$ K. Brismar*2; T. Tuvemo*1; J. Gustafsson ${ }^{1}$

${ }^{1}$ University Childrens Hospital, Uppsala, Sweden; ${ }^{2}$ Karolinska

Hospital, Stockholm, Sweden

Objectives: Abnormalities in the GH-IGF-I system with elevated GH levels and reduced IGF-I concentrations may be an important mechanism behind deteriorated metabolic control in adolescent IDDM girls. One way to improve glucose regulation would be to reduce excess $\mathrm{GH}$ secretion by anticholinergic therapy. The aim of this study was to evaluate the effect of three weeks of anticholinergic treatment on GH secretion, insulin sensitivity and lipolysis in adolescent girls with poorly controlled diabetes. Methods: Eleven IDDM girls in late puberty were treated with Pirenzepine (PZP), $100 \mathrm{mg}$ twice daily for three weeks. Before start, and after three weeks of PZP treatment HbA1c and IGF-I as well as 24-hour serum profiles of GH, insulin, glucose and IGFBP1 were analysed. Insulin sensitivity was evaluated with euglycaemic hyperinsulinaemic clamp technique. The rate of lipolysis was analysed by infusion of stable isoptope labelled glycerol during basal conditions $(n=11)$ and during a euglycaemic hyperinsulinaemic clamp $(n=6)$. Results: After three weeks of PZP therapy there was a significant improvement in glycaemic control (HbAlc $10.0 \pm 0.23 \%$ vs. $9.2 \pm 0.23 ; \mathrm{p}<0.0001)$. However, no difference in GH secretion or secretory pattern was observed. There was a significant increase in insulin sensitivity (glucose elimination $72.1 \pm 5.1 \mathrm{mg} / \mathrm{m}^{2} \times \min$ vs. $98.5 \pm$ $8.3 ; \mathrm{p}<0.003)$. The rate of lipolysis was unchanged after PZP treatment, but there was a clear reduction in the rate of lipolyis during hyperinsulinemic clamp. Conclusions: Anticholinergic therapy significantly improved insulin sensitivity in adolescent girls with diabetes. However, this effect did not seem to be mediated by a reduction in GH secretion or secretory pattern. Since GH stimulates lipolysis, the unchanged rate of glycerol production is in line with these findings. 
P3-521 Diabetes and Glucose Metabolism

Celiac Disease, Type 1 Diabetes and Autoimmune Thyroid Disease: A New Polyglandular Syndrome

M.C. Matteoli*1; C. Bizzarri*1; I.P. Patera*1;O. Porzio*1; G. Giannone $^{* 1} ;$ M. Cappa ${ }^{1}$; L. Lucentini*1

Dipartimento di Pediatria e Diabetologia, Ospedale Bambino Gesu,

Palidoro, Italy

An association between celiac disease (CD) and disorders of immunological aetiology has been reported in several studies. It has been demonstrated that $\mathrm{CD}$ patients are frequently affected by insulin dependent diabetes mellitus (IDDM) and autoimmune thyroid disease (ATD). We screened for CD and ATD all our IDDM population. Serum autoantibodies directed against thyroid peroxidase (TPO), thyroglobulin (HTG), gliadin (AGA), endomysium (EMA) and reticulin (ARA) were detected every six months. ATD was diagnosed in patients having persistently positive anti-thyroid antibodies (AAT) associated with clinical goiter or morphological abnormalities found at thyroid sonography. The diagnosis of CD was based on the ESPGAN criteria. The mean duration of follow up was $5 \pm 2$ years. Four hundred and twenty six IDDM patients were examined ( 221 males and 205 females, mean age $21 \pm 8$ years, mean age at diabetes onset $9.1 \pm 5$ years). AAT were persistently positive in 70/426 IDDM patients (16.4\%); 36/426 developed ATD (8.4\%). Four IDDM patient presented thyroid nodules without AAT. Forty three IDDM patients were affected by CD, 9/43 (20.9\%) presented persistent AAT, 8/43 (18.6\%) developed ATD. Two CD patients were affected by non autoimmune thyroid disease. AAT prevalence in IDDM-CD patients is higher (but not statistically different) than in IDDM patients. On the contrary ATD is significantly more frequent in IDDM-CD patients than in IDDM patients $(18.6 \%$ versus $8.4 \%, \mathrm{p}<$ $0.005)$. Thirty six subjects between AAT positive. IDDM patients $(51 \%)$ developed ATD whereas in the group of AAT positive-IDDM-CD patients $89 \%$ developed ATD. In conclusion AAT positive predictive value is higher in IDDM-CD patients. EMA persisted at significant titres in 50\% of IDDM-CDATD patients, after gluten withdrawal. Since the prevalence of ATD is higher in IDDM-CD patients, we can suppose that poor compliance with the gluten free diet act as a likely determinant on the onset of ATD in this group of subjects.

P3-522 Diabetes and Glucose Metabolism

From Incidental Hyperglycaemia to Maturity Onset Diabetes of the Young (MODY): 2 Case Reports

G. d'Annunzio*1;A. Alibrandi*1; M.A. Avanzini*1; F. Barbetti*3; M. Martinetti* ${ }^{2} ;$ B. Salati*1; R. Lorini ${ }^{4}$

1 Department of Paediatrics and ${ }^{2}$ Immunohaematology and

Transfusion Center, IRCCS Policlinico S. Matteo, Pavia, Italy; ${ }^{3}$ Unit of Molecular Pathology of Diabetes, IRCCS San Raffaele Hospital, Milan, Italy; ${ }^{4}$ Department of Paediatrics, G. Gaslini Institute, Genoa, Italy

Differential diagnosis of incidental hyperglycaemia $(>100 \mathrm{mg} / \mathrm{dl})(\mathrm{IH})$ includes MODY, an heterogeneous disease characterised by autosomal dominant inheritance, juvenile onset ( $<25$ years of age), non-insulin dependence, absence of ketosis. Among the 58 subjects with IH followed at our Department ( $40 \mathrm{M}$ and $18 \mathrm{~F}), 20$ showed persistent fasting hyperglycaemia, family history of type 2 diabetes, and absence of autoantibodies. Based on these data MODY was suspected, up to now confirmed by genetic analysis in II cases (8 MODY-2, 1 MODY-3, 2 MODY-not $2 /$ not3). Case n.1. R., with a maternal axis positive for type 2 diabetes, showed hyperglycaemia $(130 \mathrm{mg} / \mathrm{dl})$ at 3.2 years of age, then confirmed in absence of polyuria, polydypsia, weight loss and autoantibodies against $\beta$-cell (GADA, IA-2A). Regular growth and BMI were observed. Oral glucose tolerance test (OGTT) and intravenous glucose tolerance test (IVGTT) were normal. Genetic analysis showed mutation of the glucokinase gene (MODY-2). During follow-up, all clinical examinations were unremarkable, the child actually follows a normocaloric diet without simple sugars. Degree of metabolic control is good (HbA1c mean levels 5.8\%; n.v. 3.5-5.8\%). Case n.2. $\mathrm{J}$, an 11.3 year old girl, suffering from coeliac disease and following a glutenfree diet, showed IH during an admission to the hospital because of orthopedic surgery. Growth was higher than 97th percentile according to Tanner and BMI was 21.8 . Familial history reported type 2 diabetes mellitus in both parents' relatives. Hyperglycaemia was confirmed, in absence of both GADA and IA$2 \mathrm{~A}$. IVGTT showed first phase insulin response below the 1st percentile and OGTT was compatible with diabetes mellitus. HLA typing was A33,2; CW7,X; B49(W4),45(W6); DR11(5),4; DQ3,3. The girl was prescribed a simple sugarand gluten-free diet, and blood glucose levels returned to normal levels. Genetic analysis showed mutation in HNF-1 $\alpha$ gene, compatible with MODY-3. During follow-up, overnight albumin excretion rate and eye fundus examination were normal. The girl was taught about self-management of diabetes, regularly performed, and the degree of metabolic control is good (HbAlc mean levels 5.6\%). An IH with a familial history for type 2 diabetes mellitus could be a MODY. Since each form of MODY has different prognosis and outcome, a firm genetic diagnosis is mandatory for correct therapy as well as follow-up of vascular complications.

P3-523 Diabetes and Glucose Metabolism

\section{Diabetes Type 1 in Children under Five: Multicentre Study}

M. Chueca*1; M. Oyarzabal1; A. Sola*1; M. Aliaga*1; M.J. Lopez*2 ; C. Luzuriaga*3;I. Rica*4; M. Rodriguez*5

Pediatric Endocrinology Units, ${ }^{1}$ Pamplona, ${ }^{2}$ Valencia, ${ }^{3}$ Santander,

${ }^{4}$ Bilbao, Spain; ${ }^{5}$ Zaragoza Hospital, Zaragoza, Spain

Onset of diabetes type 1 occurs in early infancy in a significant number of patients. Recent epidemiologic studies show a peak incidence in this age group in which difficulties in management of the disease increase. Objectives: To compare the characteristics of children in this age group at disease onset between 1995 and 1999, and analyse metabolic control, acute complications and treatment regimen in both groups. Material and Methods: From January 1,1995 to December $31,1999,312$ children at the different centres suffered the onset of diabetes: $n=60<5$ years and $n=252$ between 5 and 15 years. Data analysed at onset are: clinical (Ketoacidosis [KAD]) and metabolic (basal and post-glucagon C-peptide) aspects; and during evolution: insulin treatment (number of injections and insulin doses [IU/ $\mathrm{kg} / \mathrm{d}]$, use of Lyspro), metabolic control (HbAlc) and acute complications (severe hypoglycaemias [HS] and KAD). Results: Nineteen percent of diabetes onsets correspond to the $<5$ years group; the Hospital of Valencia presented the most patients in this group $(56 \%)$ and the Hospital of Santander the least $(12 \%)\left[\chi^{2}: 13.9, p<0.01\right]$. No significant increase was observed over these years in this age group, with $\mathrm{C}$ peptide being lower $(\mathrm{p}<0.01)$ and onset more often in KAD $\mathrm{n}=30,52 \%\left(\chi^{2}\right.$ : $6.23 ; \mathrm{p}<0.05)$. HS were more frequent during evolution in younger patients (11 with 13 HS vs. 5 with $5 \mathrm{HS},\left[\chi^{2}: 9.71 ; \mathrm{p}<0.01\right]$, with similar metabolic control), but not KAD; no differences were found in the remaining variables studied, except in the dose of insulin $/ \mathrm{kg} /$ day which was lower in those $<5$ years/ $\mathrm{t}=2.33 ; \mathrm{p}<0.05$ ); the latter follows a regimen of 3 injections in $51 \%$ of cases and 2 in $42 \%$, compared with $61 \%$ with 3 injections and $27 \%$ with 2 in those over 5 years of age. Conclusions: 1 ) Children under 5 with diabetes constitute an important group, with greater severity and more ketoacidosis at onset. 2) Severe hypoglycaemias in this age group must be particularly warned against and forseen because of possible repercussions in cognitive development. 
P3-524 Diabetes and Glucose Metabolism

Atypical Course of IDDM in Children with Trisomy 21 M. Korpal-Szczyrska*1; H. Kaminska*1;D. Birholz*1; B. Dorant*1; Introduced by B. Rymkiewicz-Kluczynska

1 Pediatric Endocrinology and Diabetology Department, Medical

University, Gdánsk, Poland

The incidence of insulin dependent diabetes mellitus (IDDM) in trisomy 21 patients is higher than in the general population, but this association has rarely been reported. Nonketonic hyperosmolar diabetes coma is rare during childhood. This syndrome is associated with a $20-70 \%$ mortality rate. In the last 2 years in our department 3 children ( 2 girls and 1 boy aged 8-13 yr) with trisomy 21 and newly diagnosed IDDM were observed. At admission the diagnosis of hypermolar nonketotic coma was made on the basis of the physical findings and laboratory results. The children were in a coma, severely dehydrated and hypotensive. Laboratory findings revealed hyperosmolality in ranges $350-423 \mathrm{mmol} / \mathrm{kg}$, hyperglycaemia (60-135 mmol/l), sodium levels at $150-180 \mathrm{mmol} / 1$ and BUN $18-114 \mathrm{mmol} / \mathrm{l}$. In none of them ketoacidosis was noted ( $\mathrm{pH}$ 7.3-7.4). The guiding principles of acute period therapy in all children were rehydratation, which was more intensive than in patients with ketotic coma $(140 \mathrm{ml} / \mathrm{kg} / 24 \mathrm{~h})$ and a low dose of insulin infusion $(0.05 \mathrm{U} / \mathrm{kg} / 24 \mathrm{~h})$ given within $72 \mathrm{~h}$. Glycemia levels returned to normal in all patients after $36 \mathrm{~h}$, plasma sodium after $72 \mathrm{~h}$ and osmolality after $96 \mathrm{~h}$ of the treatment. In one of the patients (13 yr girl) the treatment was complicated on the third day by acute renal failure (creatinine level $6.4 \mathrm{mg} \%$ ) and respiratory failure. She needed hemodialysis and controlled ventilation for four days, after which normalisation of renal function was observed (creatinine level $0.7 \mathrm{mg} \%$ ). After the acute period all children were discharged from hospital in a good condition. The further course of IDDM in children with trisomy 21 was mild. In all of them diabetes is well-controlled inspite of the educational difficulties. $\mathrm{HbAlC}$ is below $7 \%$ in all of them, postprandial glycemia levels are below $10.5 \mathrm{mmol} / 1$. Their insulin requirements are very low $(0.3 \mathrm{U} / \mathrm{kg} / 24 \mathrm{~h})$. Evaluation of $\mathrm{C}$ peptide showed a level 0.44 $0.98 \mathrm{ng} / \mathrm{ml}$. Conclusions: The clinical characteristics of IDDM in children with trisomy 21 is different from subjects with classical IDDM. Hyperosmolar non ketotic coma as the initial manifestation of IDDM must be kept in mind especially in children with trisomy 21 . After the acute period diabetes in children with trisomy 21 is fairly easy to control.

\section{P2-525 Water Metabolism}

\section{Chronic Hyponatremia Due to an Abnormal Secretion of Antidiuretic Hormone (ADH)}

G.-A. Loeuille ${ }^{* 1} ;$ P. Tilmont*2; A. Savage*3; M.C. Vantyghem*4; Introduced by M.G. Forest

${ }^{1}$ Dunkerque, France; ${ }^{2}$ Calais Hospital, Calais, France; ${ }^{3}$ Dunkerque

Hospital, Dunkerque, France; ${ }^{4}$ Lille, France

Chronic hyponatremia $(\mathrm{CH})$ is a rare condition. We present hereby a patient with this disorder. Case Report: The boy was born after an uneventful gestation at 34 weeks of pregnancy. The infant had a midline cleft lip with no other abnormality. $\mathrm{He}$ did not have polydipsia nor polyuria. He presented constantly with $\mathrm{CH}$. He had normal renal, adrenal (cortisol, aldosterone, ACTH test) and thyroid (FT4, T3 and TSH) functions. Plasma renin activity was suppressed and serum levels of urea and urate were decreased. Detailed evaluation of this $\mathrm{CH}(119$ to $134 \mathrm{mmol} / \mathrm{l})$ with corresponding hypoosmolality ( 240 to $279 \mathrm{mosm} / \mathrm{kg}$ ) was performed at age $3,8,12$ years. Physical abnormality was confined to a microcephalia ( $-3,8$ SD). Magnetic resonance imaging displayed normal anatomy of the midline structures. Psychometric testing showed a quotient of 66 on the Brunet-Lézine intelligence scale. Investigation of Osmoregulation: Several testings were performed during a 10 day period, that is hypertonic saline solution infusion, water deprivation test with short desmopressin test, and standard water load. The child was fed first with a low sodium diet then with a supplementation of salt. Results: The patient responded appropriately to fluid restriction, fluid loading and sodium loading while maintaining serum sodium concentrations at low levels. Urinary osmolality and $\mathrm{ADH}$ concentrations were abnormally high when related to the hypotonicity of serum observed. Parental injection of DDAVP provoked a correct maximal renal concentration capacity. Plasma levels of atrial natriuretic peptide (ANP) were not increased. The normal regulation of serum tonicity took place at a lower osmolality threshold $(238 \pm 9 \mathrm{mosm} / \mathrm{kg})$ but the set point of the osmoreceptor may be changing or shifting, depending on time. Conclusion: The mechanism for the resetting of the osmostat is not understood: unlike other pediatric cases, our patient had no demonstrable lesion in central nervous system, and moreover we could exclude an abnormal ANP control.
P2-526 Water Metabolism

Essential Hypernatremia: Relapse Due to Withdraw of Chlorpropamide

E. Piantanida*1; A. Salvatoni*1;C. Orsatti*1; R. Rovelli*1;

R. Cardani*1; L. Nespoli*1; Introduced by P.Brambilla

${ }^{1}$ Insubria University, Varese, Italy

We present a case (F.S.) of a 11 years old boy with severe spastic quadriplegia, mental retardation and essential hypernatremia due to the outcome of a neurosurgical intervention for the removal of an astrocytoma of the third ventricle. Moreover impairment of body temperature and blood pressure regulation were occasionally observed. The initial diagnosis of essential hypernatremia was suspected at 2.8 years old ( 10 months after the intervention), on the basis of severe hypernatremia $(191 \mathrm{mEq} / \mathrm{l})$ resistant to DDAVP treatment, in absence of polyuria, weight loss and dehydration. ADH plasma level was in the normal-low range. Treatment with chlorpropamide was started $\left(60 \mathrm{mg} / \mathrm{m}^{2} /\right.$ dose twice a day) and natremic levels normalised within ten days. Following a hypoglycaemic event the dosage was reduced to $40 \mathrm{mg} / \mathrm{m}^{2} /$ dose twice a day. The patient was maintained in a prolonged treatment with chlorpropamide and natremia was constantly normal. After eight years the therapy was discontinued by the parents who considered it an unnecessary treatment. Three months later the patient was referred to the hospital because of swelling of the foot and irritability and severe hypernatremia $(195 \mathrm{mEq} / \mathrm{l})$ was demonstrated. The reintroduction of chlorpropamide was effective once again in reversing both symptoms and hypernatremia. Comment: Dose, frequency of administration and duration of chlorpropamide treatment for essential hypernatriemia is undefined. The present case confirms the effectiveness of chlorpropamide $(40 \mathrm{mg} / \mathrm{sqm} /$ dose twice a day) in acute and chronic treatment of hypernatriemia and shows the need for a life-long therapy.

P2-527 Water Metabolism

Reduced Processing and Endoplasmic Reticulum Retention of the AVP Prohormone by a TYR2HIS FNDI Causing Mutation of the AVP-NPII Gene

J. Hvarregaard*1; C. Siggaard*1; T.J. Corydon*2;M. Ozata*3;

N. Gregersen $^{*} 1$; L. Bolund ${ }^{* 2}$;S. Rittig*4; Introduced by $O$. Wolthers ${ }^{1}$ Aarhus University Hospital, Aarhus, Denmark; ${ }^{2}$ Aarhus University, Aarhus, Denmark; ${ }^{3}$ Gulhane School of Medicine, Ankara, Turkey;

${ }^{4}$ Aarhus, Denmark

Autosomal dominant familial neurohypophyseal diabetes insipidus (FNDI) is characterized by polyuria, polydipsia, and a deficient neurosecretion of the antidiuretic hormone, arginine vasopressin (AVP) and is caused by different mutations of the AVP-NPII gene. Purpose: To characterize the molecular mechanisms underlying the development of FNDI in a Turkish family carrying a T285C missense mutation in the AVP-NPII gene predicting a substitution of Tyr2 with His in the prohormone vasopressin moiety. Methods: A pcDNA3.1 plasmid containing a human wild-type or mutant AVP-NPII cDNA was transiently transfected into human teratocarcinoma Ntera2/D1 cells. Cell proteins were metabolically labeled, immunoprecipitated with rabbit anti-neurophysinII (NPII) or anti-vasopressin from cell lysates and medium, separated by SDS-PAGE, and analyzed. Cells were immunostained with rabbit anti-NPII and analyzed by confocal laser scanning microscopy. Co-localization with the endoplasmic reticulum (ER) was examined by staining with mouse anti-Grp78 recognizing the ER retention signal KDEL. Results: Ntera2/D1 cells expressing mutated AVP-NPII cDNA showed reduced ability to process the AVP prohormone compared to the wild-type cells, which on the other hand were capable of synthesizing AVP prohormone, partially processing it into vasopressin-neurophysin, and secreting both partially processed and unprocessed precursor hormone into the cell medium. Although a small amount of mutant prohormone was processed and subsequently secreted constitutively, the majority of mutant prohormone remained unprocessed and seemed to be retained exclusively within the cells. Immunostaining of the cells demonstrated that colocalization of anti-NPII reactive protein and the ER was profound in cells expressing the mutant AVP prohormone but not in those expressing the wild-type. Conclusion: Substitution of Tyr2 with His in the vasopressin moiety of the AVP prohormone does not seem to interfere with its synthesis but severely inhibits the intracellular processing and transport. These results support biochemical evidence that Tyr2 of the AVP hormone is important for proper AVP binding to NPII and for subsequent folding and dimerization of the precursor protein. Furthermore, the study supports the hypothesis that FNDI is caused by intracellular retention of abnormally folded precursor protein. 
P2-528 Water Metabolism

Morbidity and Mortality Associated with Vasopressin Replacement Therapy

V. Rizzo ${ }^{1} ;$ A. Albanese ${ }^{1} ;$ R. Stanhope ${ }^{1}$

1 Department of Paediatric Endocrinology, Great Ormond Street Hospital for Children and the Middlesex Hospital, London, United Kingdom

Objective: To asses the risk factors for adverse effects associated with DDAVP replacement treatment and to compare different routes of DDAVP administration. Patients and Methods: We have assessed 103 children (44 F, $59 \mathrm{M}$ ) with cranial diabetes insipidus (DI). The commonest aetiology was craniopharyngioma $(n=56)$. Mean age was 6.9 years (range $0.1-17.6)$ and follow-up was for a mean of 5.2 years (range 0.1-16.8). Intramuscular (IM) DDAVP was administered to 59 patients in the immediate postoperative period; 84 patients received intranasal (IN) DDAVP and 64 the oral (PO) formulation. 42 patients treated with PO DDAVP had been previoulsy treated with the in preparation. Results: Eight patients died during the follow-up. In at least two of them, this was due to documented water intoxication. Major complications of DDAVP therapy (symptomatic water overload with or without seizures) or asymptomatic hyponatraemia were observed in 38 patients. A significant higher incidence of total complications $(\mathrm{p}<0.01)$ was seen among 86 ACTH deficient patients, while receiving hydrocortisone replacement treatment. Major complications also occured more frequently $(\mathrm{p}<0.01)$ in patients on concomitant carbamazepine treatment. Incidence of side-effects was not different in patients treated with IM, IN or PO DDAVP. Conclusion: In cortisol deficient patients inadequate cortisol replacement therapy may increase the risk of water overload. The role of carbamazepine in potentiating the antidiuretic actin of DDAVP needs to be considered. Special caution is needed in managing patients who have both anterior and posterior pituitary failure, particularly during acute illness.

P2-529 Endocrine Consequences of Cancer

\section{Bone Mineral Density in Long-Term Survivors of}

Childhood Malignancies: Comparison between Haematological and Brain Tumours

A. Corrias ${ }^{* 1}$; S. Bertelloni ${ }^{2}$; R. Lala*1; G.I. Baroncelli*2 ; F. lanni*3. M. Andreo $* 1 ;$ G. Saggese 2

1 Paediatric Endocrinology, Regina Margherita Paediatric Hospital,

Torino, ${ }^{2}$ Division of Paediatrics, University Santa Chiara Hospital,

Pisa, ${ }^{3}$ Division of Endocrinology, V. Cervello Hospital, Palermo, Italy

Background: Low bone mineral density (BMD) may be a late effect of childhood cancer. Objective: To evaluate BMD after the attainment of final height in long-term survivors of paediatric haematological or brain tumours. Patients and Methods: Sixteen adolescents or young adults treated in childhood for cancer were studied. Patients were subdivided as group $1=$ haematological malignancies [mean age $19.5 \pm 2.8$ years $(\mathrm{ALL}, \mathrm{n}=5$; lymphoma, $\mathrm{n}=$ 5)] and group $2=$ brain tumours [mean age $19.6 \pm 3.4$ years (medulloblastoma, $\mathrm{n}=3$; ependymoma, $\mathrm{n}=2$; glioma in neurofibromatosis, $\mathrm{n}=1)]$. Areal BMD (aBMD) was measured at lumbar spine (L2-L4) by DXA and volumetric BMD (vBMD) was calculated. Data are in SDS according to age and sex. Results: $\mathrm{aBMD}$ and $\mathrm{vBMD}$ were reduced in both groups (table).

\begin{tabular}{crlll}
\hline & $\mathrm{n}$ & $\mathrm{M} / \mathrm{F}$ & $\mathrm{aBMD}$ & $\mathrm{vBMD}$ \\
\hline Group 1 & 10 & $6 / 4$ & $-2.7 \pm 2.7^{*}$ & $-2.4 \pm 3.0^{*}$ \\
Group 2 & 6 & $5 / 1$ & $-6.1 \pm 3.2^{* *}$ & $-1.9 \pm 4.2^{* * *}$ \\
\hline$* \mathrm{p}<0.02$ vs. $0 ;{ }^{* *} \mathrm{p}<0.04$ vs. 0 and $\mathrm{p}<0.05$ vs. gr. $1 ; * * * \mathrm{p}=$ NS vs. 0.
\end{tabular}

aBMD values were significantly reduced in group 1 in comparison with those of group 2, while vBMD values were not (table). Conclusions: In adolescent and young adult survivors of paediatric malignancies, lumbar aBMD and vBMD is reduced. The finding of lower aBMD in group 2 in comparison with group 1, while vBMD is not, might be explained by an impaired growth of vertebral bodies as a late effect of the high cranial irradiation in the former group.
P2-530 Endocrine Consequences of Cancer

Growth and Height in Children and Adolescents with

Bone Tumours: Effect of Chemotherapy and

Relationship to the Genotype for the Vitamin D Receptor Gene

E. Ruza*1; L. Sierrasesumaga*1;E. Sotillo*1; A. Patiño*1; C. Azcona ${ }^{1}$

Pamplona, Spain

Introduction: Association between tall stature and increased risk of developing bone tumours is, to date, a controversial matter. Nevertheless, it is widely accepted that tissues of fast turnover are particularly susceptible to cancer development. The Vitamin D Receptor gene (VDR) is involved in bone development and metabolism. Several polymorphisms in the coding sequence of this gene have been associated with bone mass acquisition and, more recently, with tall stature. The adverse effect of the chemotherapy overgrowth in children with cancer is well documented, and this effect is even more severe if chemotherapy is administered throughout the pubertal growth spurt. Objectives: (1) To establish if height at diagnosis in children and adolescents with bone tumours differs from the control group. (2) To characterise the polymorphism of the VDR gene (FokI) that has been associated with tall stature. (3) To evaluate the effect of chemotherapy over the growth rate in patients with bone tumours. Materials and Methods: DNA was obtained from 111 paediatric patients with bone tumours (63 osteosarcomas (OS) and 48 Ewings Sarcomas (ES)) and from 88 healthy children. Height measurements have been adjusted by sex and age and converted into SDS (Standard Deviation Score) according to the British 1990 growth standards. Results were expressed as Mean (Confidence Interval), M (CI). The FokI polymorphism of the VDR gene was analysed by endonuclease analysis and genotypes were established $(\mathrm{FF}=\mathrm{CC}, \mathrm{Ff}=$ $\mathrm{CT}$ and $\mathrm{ff}=\mathrm{TT})$. Results: Patients with OS and ES are taller at diagnosis than controls (OS, $0.35(0.005 ; 0.6)$; ES, $0.56(0.13 ; 0.98))$. An increased frequency of the Ff genotype $(p=0.002)$ has been detected in the OS group. This genotype has been found to be more frequent among tall healthy individuals. Height gain SDS is statistically diminished in patients with bone tumours undergoing chemotherapy regimens $(-0.35(-0.50 ;-0.2))$. This reduction is specially marked in patients within the age range from 9 to 14 years $(-0.6(-0.78 ;-0.43)$ compared to the others $(-0.14(-0.36 ;-0.09))$. Conclusions: Patients diagnosed with OS or ES are taller at diagnosis than healthy children, age and sexmatched. This may support the hypothesis that the taller children, or those with higher growth rates, may beat risk for developing bone tumours. The growth rate of children undergoing antitumoral treatments is decreased, which suggests that chemotherapy agents induce growth inhibition that is specially noticeable when the pubertal growth spurt takes place. 
P2-531 Endocrine Consequences of Cancer

\section{Height in Patients Treated for Acute Lymphoblastic Leukaemia (ALL). A Meta-analysis of the Years 1966-1998}

J.H. Brämswig ${ }^{1}$; K.A. Bergmann*1; K. Kiese*1; A. Heinecke*1 ${ }^{1}$ University Childrens Hospital and Department of Biomathematics, Münster, Germany

Introduction: Normal height as well as short stature have been reported in patiens treated for ALL Objective: To evaluate the effects of different treatment modalities on height in ALL patients by reviewing published data of the years 1966-1998. Methods: We explored the databases Medline and Current Contents for studies reporting height-SDS (HSDS) of ALL patients during the years 1966-1998. A random-effects meta-analysis (J.L. Fleiss 1993) was performed to calculate the effect sizes and confidence intervals of the HSDS with respect to different doses of cranial irradiation. The results at the end of therapy and at adult height were compared to the HSDS before therapy and to the population mean. Results: Between the start and the end of therapy there is a significant loss of the effect sizes of HSDS in all three treatment groups with -0.27 SDS,-0.51 SDS and -0.76 SDS in patients receiving $0 \mathrm{~Gy}, 18-20 \mathrm{~Gy}$ or 24-25 Gy cranial irradiation. The loss of HSDS increases further to -0.49 SDS, -0.71 SDS and -1.28 SDS in each respective group, when adult height is reached. The effect size of HSDS, when compared to the population mean, is normal at diagnosis with +0.02 SDS $(0 \mathrm{~Gy})$ or significantly above normal with +0.17 SDS (18-20 Gy) and + 0.27 SDS (24-25 Gy). At the end of therapy HSDS is still within the normal population range irrespective of the dose of cranial irradiation. Final height is normal in patients with $0 \mathrm{~Gy}$ and $18-20 \mathrm{~Gy}$, but significantly below the population mean with -0.59 SDS (CI -0.94 to -0.13 $\mathrm{SDS}$ ) in the $24 \mathrm{~Gy}$ irradiation group resulting in a mean height difference of $2.8 \mathrm{~cm}$ in girls and $3.5 \mathrm{~cm}$ in boys when compared to the population mean. Conclusions: Children with ALL have a significant height loss at the end of therapy and at adult height when HSDS is compared to HSDS before therapy. Comparing adult height to the population mean only high doses of cranial irradiation (24-25 Gy) have a significant impact on final height. For most patients this height loss is clinically irrelevant.

P2-532 Endocrine Consequences of Cancer

\section{Endocrine Late Effects after BMT in Children and} Adolescents

C.P. Schwarze ${ }^{* 1} ;$ R. Dopfer ${ }^{* 1} ;$ T. Klingebiel ${ }^{* 1} ;$ D. Niethammer ${ }^{* 1}$; D. Fuchs*2;C. Vilser*2; L. Patzer*2; F. Zintl*2;C. Bender-Götze*3. E. Frey ${ }^{*} ;$ C. Peters ${ }^{* 4} ;$ H. Gadner ${ }^{* 4}$;M.B. Ranke

University Children's Hospitals, 1 Tübingen, ${ }^{2}$ Jena, ${ }^{3}$ München,

Germany; ${ }^{4}$ St. Anna Kinderspital, Vienna, Austria

Objective: To evaluate late effects concerning growth and endocrine function in children and adolescents after bone marrow transplantation (BMT). Patients and Methods: 318 patients treated with BMT from 1996 to 1999 were investigated for late effects. The underlying diseases were ALL 21\%, AML $13 \%$, CML 7\%, MDS 8\%, lymphoma 4\%, aplastic anaemia $8 \%$, neuroblastoma $11 \%$, solid tumours $15 \%$ and others $13 \%$. They were followed-up according to a standardised protocol: determination of weight, height (HT), sitting height, leg length, arm-span, head circumference and pubertal status. Analysis of TSH, T 4 and $\mathrm{fT}_{4}, \mathrm{LH}, \mathrm{FSH}$, estradiol and testosterone, in addition, TRH and GnRH testing was performed. Evaluation of the $\mathrm{GH}$ axis included measurement of IGF-I and IGFBP-3 and an arginine test. Patients were examined before BMT and 3, 6, 12, 24 and 36 months after BMT. Results: HT-SDS before BMT was significantly, $\mathrm{p}<0.005$, reduced compared to target HT $(\mathrm{n}=145)$ at -0.52 SDS (median) (range -4.02 to 2.71 ) versus 0.11 SDS $(-2.43$ to 2.18$)$. HT-SDS significantly decreased further to -0.67 SDS $(-5.03$ to 2.94$), \mathrm{p}<0.05$, and -1.03 SDS ( -4.17 to 0.16$), \mathrm{p}<0.05$, after 24 and 36 months. IGF-I and IGFBP-3 had no predictive value for changes in HT-SDS. Individual changes in HT-SDS ranged from -2.02 to 1.93 after 12 to 36 months. Growth hormone deficiency was diagnosed in 6 patients. Before BMT, primary hypothyroidism was seen in $13.4 \%(23 / 172)$ and after BMT in $18.7 \%(44 / 235)$. Central hypothyroidism was suspected in $64.4 \%$ (96/149) with low values for iT4 and/or T4 and a 'normal' TSH. This was confirmed by low TSH peaks in $25 / 119$ of the TRH tests. After
BMT, 64.9\% (124/191) had low iT4 and/or T4 levels and a 'normal' TSH. Thyroxin was substituted in 15 patients. Hypergonadotropic hypogonadism was detected in $21.9 \%(14 / 64)$ female (f) and $17.7 \%(26 / 147)$ male (m) patients before BMT. GnRH tests, however, revealed a much higher rate of gonadal dysfunction: $50.0 \%(32 / 64)$ and $27.9 \%(41 / 147)$, respectively. Longitudinal data in $28 \mathrm{f}$ and $73 \mathrm{~m}$ patients showed normal LH/FSH levels before and elevated levels after BMT in $28.6 \% \mathrm{f}$ and $22.0 \% \mathrm{~m}$ patients. Replacement therapy was given in 8 patients. Conclusions: Growth failure and endocrine deficiencies are very frequent after BMT. The right diagnostic tools have to be applied for detection of developing dysfunction. The time and order of their occurrence cannot be predicted. To ensure normal growth and development each patient has to be followed individually. Investigations must be performed according to a standardised protocol with co-operation of the paediatric haematologist and the paediatric endocrinologist.

P2-533 Endocrine Consequences of Cancer

\section{Irradiation Damage to Hypothalamic Control of Water} Balance

V. Gorenberg ${ }^{* 1} ;$ Z. Hochberg ${ }^{1}$

${ }^{1}$ Rambam Medical Center, Haifa, Israel

Therapeutic irradiation of patients with brain tumors results in hypothalamic damage to the control of anterior pituitary functions. Water balance is regulated from nearby centers and includes vasopressin (VP) synthesis and release, osmotic control and thirst sensation. To evaluate possible damage to water balance, 4 patients aged 10-22 y, with proven hypothalamic damage (GH-deficiency, $n=4$ and central precocious puberty, $n=2$ ) $6-15$ y after irradiation, but normal adrenal and thyroid functions, were studies and compared to 6 normal age-matched controls. To evaluate susceptibility to extreme changes, they were studied after $14 \mathrm{~h}$ fast and after a water load $(20 \mathrm{ml} / \mathrm{kg})$. To determine the osmotic threshold for VP release, the water load was followed by hypertonic $\mathrm{NaCl}$ infusion under conditions of maintained high plasma volume. Threshold was defined by a sudden drop in free water clearance (FWC). After $14 \mathrm{~h}$ water deprivation, plasma and urinary osmolality (Posm/Uosm) were $293 \pm 6 / 917$ $\pm 73 \mathrm{mosm} / \mathrm{kg}$ in the Pts and $298 \pm 6 / 852 \pm 79 \mathrm{mosm} / \mathrm{kg}$ in Ctr (n.s.). FWC at that point was $-0.2 \pm 0.8 \mathrm{ml} / \mathrm{min}$. In the Pts $83 \pm 9 \%$ of the water load was excreted within $3 \mathrm{~h}$ and in $\mathrm{Ctr} 72 \pm 16 \%$ (n.s.). The osmotic threshold for VP release was $290.5 \pm 1.3$ in the Pts and $286.7 \pm 1 \mathrm{mosm} / \mathrm{kg}$ in $\mathrm{Ctr}(\mathrm{p}<0.0001)$. During $\mathrm{NaCl}$ infusion, with Posm as high as $295-302 \mathrm{mosm} / \mathrm{kg}$, thirst was denied in 3/4 patients whereas Ctr reported thirst at Posm 291-294 mosm $/ \mathrm{kg}$. Maximal Uosm was $917 \pm 73$ and $764 \pm 118 \mathrm{mosm} / \mathrm{kg}$ in Pts and Ctr resp. (n.s.). It is concluded that therapeutic irradiation may cause 6-15 years later mild hypothalamic damage in water regulation, insensitivity to thirst and mild increase in osmotic threshold. Pts have to be aware of inadvertent change in tonicity and susceptibility. 
P2-534 Endocrine Consequences of Cancer

Evaluation of Total Body Irradiation (TBI) or Busulfan Conditioning Regimens on the Thyroid Function after Bone Marrow Transplantation

B. Lebon-Labich ${ }^{* 1}$; L. Clement*2;F. Gebhard*2; P. Bordigoni ${ }^{2}$;

B. Leheup ${ }^{2}$

${ }^{1} \mathrm{CHU}$ de Brabois, Vandoeuvre les Nancy, France; ${ }^{2} \mathrm{CHU}$ de Nancy,

Vandoeuvre les Nancy, France

The incidence of thyroid abnormalities in relation to total body irradiation (TBI) or Busulfan conditioning regimens after bone marrow transplantation (BMT) was evaluated in a series of 64 patients among a cohort of 95 followed up in the paediatric haematology department. The study was retrospective. 27 patients received an autologous BMT and 37 an allogenic BMT. These patients received BMT for haematologic $(n=54)$ or metabolic or immune disorders $(n=$ 10). Pre-BMT conditioning consisted of TBI in 28 patients $(44 \%)$ and Busulfan in 36 patients $(56 \%)$. Median follow-up period after BMT was 6.4 years (range 0.6 to 14.9 years). Thyroid dysfunction was seen in 19 patients $(30 \%)$, of whom 2 exhibited transient dysfunction. Of these, 12 patients had normal thyroid function before BMT, 3 patients had thyroid dysfunction, and there was no information about pre BMT thyroid function for 4 patients. 17 patients developed compensated hypothyroidism (elevated TSH with normal thyroxine index). Two patients developed a true primary hypothyroidism. Thyroid dysfunction was diagnosed 0.7 to 4.5 years after BMT (mean: 2.2 years). 15 patients still receive thyroid hormone replacement therapy. The incidence of elevated TSH was $12 / 28(43 \%)$ in the patients who received TBI and $7 / 36$ $(19 \%)$ in those who received Busulfan. The difference between the two groups is significant $(\mathrm{p}=0.04)$. There was no difference between those two groups concerning the pre-BMT thyroid function. We did not find any difference between autologous and allogenic BMT. In conclusion, thyroid dysfunction is seen in $30 \%$ of patients after BMT. Its prevalence is significantly higher after TBI than Busulfan conditioning. The place of thyroid substitution is still questionable regarding its long term effect on the prevention of secondary thyroid tumor.

P2-535 Endocrine Consequences of Cancer

\section{Gonadal Status in Male Survivors of Childhood Brain Tumors Treated with Radiotherapy (RT) +/- Chemotherapy (CT)}

M. Schmiegelow*1; S. Lassen*1; H.S. Poulsen*1;

K. Schmiegelow*1; H. Hertz ${ }^{* 1} ;$ J. Müller $^{1}$

${ }^{1}$ Rigshospitalet, Copenhagen, Denmark

Objectives: The effect of radiotherapy (RT) and chemotherapy (CT) on gonadal function was assessed in males treated with radiotherapy (RT) +/- chemotherapy (CT) for a childhood brain tumor not directly involving the hypothalamus/pituitary (HP) axis. Methods: All males $<15$ years at diagnosis and diagnosed from January 1970 through February 1997 in the Eastern part of Denmark and less than or equal to 18 years at time of follow-up. Thirty males fulfilled the criteria. The median age at time of RT was 9.0 years (range: 0.8 to 14.9 years) and length of follow-up was 18 years (range: 2.0 to 28.0 years). All thirteen children who were treated with $\mathrm{CT}$ received alkylating agents. RT was expressed in Gy as the biological effective dose (BED), which was determined to the HP region and to the spine. BED gives a means of expressing the biological effect of RT on normal tissue in a uniform way. Levels of serum FSH, LH, testosterone, and inhibin B were measured and a gonadotropin-releasing hormone stimulation test was carried out with determination of peak FSH and LH. The gonadal hormone inhibin B exerts negative regulation of pituitary production and secretion of FSH and serves as a direct serum marker of spermatogenesis. Results: Patients treated with RT + CT $(n=13)$ compared to patients treated with RT only $(\mathrm{n}=17)$ had a significantly higher median peak FSH $(8.33$ vs. $3.79 \mathrm{IU} / 1, \mathrm{p}=0.03)$ and median peak $\mathrm{LH}(20.0 \mathrm{vs} .12 .8 \mathrm{IU} / \mathrm{l}, \mathrm{p}=0.03)$, and a significantly lower median inhibin B $(86.0$ vs. $270 \mathrm{pg} / \mathrm{ml}, \mathrm{p}=0.03)$, and inhibin $\mathrm{B} / \mathrm{FSH}$ ratio $(12.8$ vs. $107.9, \mathrm{p}=0.04)$, which indicated gonadal damage. We found a significant inverse correlation between $\mathrm{FSH}$ and inhibin $\mathrm{B}$ and $\mathrm{FSH}$ and total testicular volume ( $\mathrm{rs}=-0.84 ; \mathrm{p}<0.0001)$, $(\mathrm{rs}=-0.67 ; \mathrm{p}<0.0001)$, respectively, and a significant correlation between inhibin B and total testicular volume ( $\mathrm{rs}=0.63 ; \mathrm{p}<0.0001$ ). Stepwise backward multiple linear regression analysis showed the best-fit model to predict inhibin B included total testicular volume $(p=0.002)$ and $C T(p=0.09)$. Conclusions: We suggest that adjuvant CT may cause gonadal damage in males with brain tumours.

\section{P2-536 Endocrine Consequences of Cancer \\ Growth Hormone Treatment after Bone Marrow Transplantation: First Year Results in 9 Patients}

B. Bakker*1; W. Oostdijk1 ;W.H. Stokvis-Brantsma*1; J.M. Vossen*1; J.M. Wit

${ }^{1}$ Leiden University Medical Center, Leiden, The Netherlands

Total body irradiation (TBI), part of the conditioning regime before bone marrow transplantation (BMT) leads to growth retardation and several endocrine and functional disorders. Final height is usually compromised, especially in single-dose TBI (sTBI). It is unknown whether GH treatment can improve growth in childhood and adult stature. Objective: To evaluate the effect of recombinant human growth hormone (rhGH) on growth after BMT for haematological malignancies in the first year of therapy. Patients and Methods: BMT-recipients with growth delay (decrease in height SDS 0.5 after BMT), a height $<-2.0$ SDS or a height SDS $>1.3$ SD below the target height SDS were included into a prospective study. After a 12-hour nocturnal GH secretion profile and at least one GH-provocation test, patients received a daily dose of 4 IU/ $\mathrm{m}^{2}$ of rhGH. Growth, bone age, and progression of puberty were closely monitored. Patients who had received $\mathrm{rhGH}$ for at least one year are included in this report ( 6 girls, 4 boys). One girl had a graft-versus-host disease of the liver and intestine. GH therapy was stopped after one year because of lack of response. Because of the severe malabsorption she was not included in the group analysis. Results: Median age at BMT was 3.8 (range 1.7 to 9.8) years and median height SDS for chronological age (HSDS-CA) was $-1.0 \mathrm{SD}(-2.7$ to +0.3$)$. Of the nine patients eight had an adequate response $(20 \mathrm{mU} / \mathrm{l})$ to $\mathrm{GH}$-provocation tests, whereas one patient had an impaired response $(13.3 \mathrm{mU} / \mathrm{l})$. The median nocturnal integrated 12-hour GH concentration was $5.1 \mathrm{mU} / \mathrm{l}$ (1.5 to 7.6, all below the lower limit of the reference data). Median age at start of therapy was 10.4 (7.6 to 13.2 ) years and median HSDS-CA was -1.8 SD $(-3.5$ to -0.5$)$. At the start of rhGH two patients had entered into puberty (Tanner stage G3 and B3 respectively). In four other patients puberty started during the first year of therapy. The median increase in height velocity was $3.0 \mathrm{~cm} / \mathrm{yr}(1.6$ to 4.5$)$ and the median increase in HSDS-CA was $0.4 \mathrm{SD}(0$ to 0.7$)$. Bone age advanced 1.2 years/year $(0.0$ to 1.8$)$ before therapy and 1.0 year/year $(0.2$ to 1.5$)$ during therapy. The median increase in height SDS for bone age was 0.5 SD (0 to 1.1). Conclusion: rhGH therapy increased height velocity in all patients. Evaluation of growth in the coming years is needed to evaluate the effect on final height. 
P2-537 Endocrine Consequences of Cancer

Fatty Liver in Childhood Associated with Hypopituitarism Secondary to Hypothalamic Tumour Resection

A. Drake*1;H. Evans*2;D. Kelly*2;P. McKiernan*2;E. Crowne ${ }^{1}$

${ }^{1}$ The Royal Hospital for Sick Children, Bristol, United Kingdom;

${ }^{2}$ Birmingham Children's Hospital, Birmingham, United Kingdom

Hypothalamic tumours in childhood may present with endocrine abnormalities and hypopituitarism secondary to resection is well recognised. Hypothalamic damage may also be accompanied by hyperphagia and rapid weight gain. We present three cases of children ( 2 male, 1 female) who had surgery for hypothalamic tumours ( 2 craniopharyngioma, 1 astrocytoma) with subsequent hypopituitarism, who developed fatty livers with progressive liver disease of unknown aetiology. Case 1 (female) presented with acutely elevated intra-cranial pressure and visual impairment secondary to a craniopharyngioma. She had surgical resection at the age of eight followed by further resection 9 months later. She subsequently developed panhypopituitarism with hyperphagia, massive weight gain and chronic hypernatraemia secondary to diabetes insipidus (DI) and adipsia. She received full pituitary hormone replacement therapy, although growth hormone was withdrawn after exacerbation of glucose intolerance. Worsening hyperglycaemia and insulin resistance have required treatment with large doses of insulin. Three years post surgery she developed abnormal liver function tests, hepatosplenomegaly and thrombocytopaenia. Liver biopsy demonstrated micronodular cirrhosis and fatty infiltration. Case 2 (aged 14) had a craniopharyngioma with antecedent DI and subsequent gonadotrophin, ACTH and TSH deficiency. He had episodes of hypernatraemia and excessive weight gain post-operatively. Case 3 had neurofibromatosis type 1 and had resection of an astrocytoma aged 3. Post-operatively he developed SIADH with serum sodium values of $123-151 \mathrm{mmol} / 1$. He also gained weight rapidly, and requires replacement thyroxine and hydrocortisone. Cases 2 and 3 developed abnormal liver function and hepatomegaly on days 10 and 12 postoperatively and have severe fatty infiltration of the liver. None of the cases received excessive blood products, and their viral serology has been negative. They did not receive hepatotoxic drugs and other causes of fatty liver and cirrhosis have been excluded. The association of fatty liver with hypothalamic tumours and subsequent hypopituitarism has not previously been reported. These children do have a number of risk factors, namely obesity, insulin resistance, growth hormone deficiency and hypernatraemia. The exact mechanism however, remains unclear and needs further investigation. It is clear that children with hypopituitarism secondary to hypothalamic tumours need monitoring, as liver disease can develop and may be progressive.

P2-538 Endocrine Consequences of Cancer

Auxoendocrine Sequelae in Patients with Medulloblastoma Treated with SNC91 Schedule

S. Einaudi*1; A. Corrias*1; J. Bellone ${ }^{1} ;$ M. Andreo ${ }^{1}$; U. Ricardi*1; F. Altare ${ }^{* 1}$. A Sandri*1. C. de Sanctis*1

${ }^{1}$ Regina Margherita Hospital, Turin, Italy

We have evaluated auxological and endocrine diseases in 13 patients ( 11 males, 2 females) treated for medulloblastoma with SNC91 schedule, including, after surgery, chemotherapy and radiotherapy. The latter differs from previous protocols in: hyperfractionation (36 Gy to the craniospinal axis and a $30 \mathrm{~Gy}$ boost on posterior fossa, administered as 1 Gy per fraction twice daily) and a lower junction between cranium and spine (C5-C6 or C6-C7 level). All patients were off-therapy for 2 years at least and were relapse-free during the observation. The mean age at diagnosis was 9.19 years (range $4-14$ years) and the patients were followed for 4.5 years (range 2.5-7.6 years); 12 were pubertal and 1 prepubertal at diagnosis. Yearly we evaluated height, sitting height $(\mathrm{SH})$, subischial leg lenghth (SLL), pubertal development, F-T3, F-T4, TSH, IGF1, cortisol and prolactin (PRL) secretion. Moreover, in patients aged 9-13 we evaluated FSH, LH in both sexes, testosterone (males),17-beta-estradiol (females). In 8 cases with severely affected growth, we evaluated also GH secretion (spontaneous nocturnal secretion and/or after GHRH+arginine stimulus); in patients with abnormal pubertal development, FSH, LH secretion after LHRH stimulus. Results: Statural growth and GH secretion: In all patients but one we observed a reduction of height SDS in respect to the height SDS at diagnosis. In 5 out of the 8 patients evaluated for $\mathrm{GH}$ levels, we registered a reduced $\mathrm{GH}$ secretion with one or both tests. In the 5 patients not tested for GH secretion IGF1 were constantly normal. In 11 patients SH/SLL ratio decreased gradually. Thyroid function: we registered primary hypothyroidism in 4 cases 3.5 yrs (range 1.54.7) from radiotherapy. Pubertal development. In 2 patients (1 male and 1 female) precocious puberty occurred. One girl complained of oligomenorrhea and was treated with progestogen which restored her cyclicity. With regard to testicular volume, it was never below the 10th centile, but in 8 cases it was below the 50th. Prolactin and cortisol secretion was normal in all cases. Conclusion: SNC91 radiotherapeutic schedule, which provides hyperfractionation and a lower junction between cranium and spine, can affect statural growth and endocrine function, particularly gonads and thyroid. Our preliminary data indicate that the auxoendocrine sequelae are less frequent than with previous protocols.

P3-539 Endocrine Consequences of Cancer

\section{De Novo and Secondary Neoplasia in GH Treated} Children. Experience from KIGS

P. Wilton ${ }^{1}$; P. Larsson ${ }^{1} ; K$. Albertsson-Wikland ${ }^{2}$; P. Chatelain ${ }^{3}$. W. Cutfield ${ }^{4}$; A.C. Lindgren ${ }^{1} ; D$. Price ${ }^{6} ;$ M.B. Ranke ${ }^{5}$ on behalf of the KIGS International Board

${ }^{1}$ Pharmacia \& Upjohn, Stockholm, Sweden; ${ }^{2}$ East Hospital,

Gothenburg, Sweden: ${ }^{3}$ Hôpital Debrousse, Lyon, France;

${ }^{4}$ University of Auckland, Auckland, New Zealand; ${ }^{5}$ Kinderklinik Sek

Päd Endokrinol, Tübingen, Germany; ${ }^{6}$ University of Manchester,

Manchester, United Kingdom

Whether GH treatment can influence the development of de novo or secondary neoplasia has been a question for many years. The pharmacoepidemiological survey of KIGS is a tool to study this question. Patients: 30,679 patients representing 77,682 years of $\mathrm{GH}$ treatment is the basis for this analysis. 1,895 patients had organic GHD after treatment for a malignant tumour or leukaemia, 15,022 patients had IGHD and 3,692 had TS. Results: In 16 patients a de novo neoplasia was reported at $0.2-7.1$ years of $\mathrm{GH}$ treatment at a dose of $0.5-1.02 \mathrm{IU} / \mathrm{kg} /$ week. The frequency of tumour was $4(0.27 \% 0)$ in IGHD, 4 $(0.91 \% 0)$ in OGHD, $3(0.84 \% 0)$ in TS and $5(0.69 \%)$ in other non-GHD. Type of neoplasia was $\mathrm{CNS}=3$, leukaemia $\mathrm{n}=3$, lymphoma $\mathrm{n}=2$ and single cases of eight other neoplasias. 10/1,387 $(0.72 \%)$ patients with a cranial tumour reported a secondary neoplasia (CNS $n=9$, squamous carcinoma $n=1$ ) with duration of GH $0.2-7.4$ years. Three of the patients had optic glioma and NF-I. A further three reported a neoplasia 2-12 months after GH was discontinued. $6 / 508(1.2 \%)$ patients with leukaemia as the reason for OGHD reported a secondary neoplasia (CNS n $=4$, squamous carcinoma and other type of leukaemia one each). In addition malignant melanoma and thyroid cancer were reported 2-3 years after discontinuation of GH. Conclusion: $1.1 \%$ of secondary neoplasia is in accordance with published data in survivors of neoplasia not treated with GH. The number of de novo neoplasia is still to few to calculate a relative risk compared to general population. Further follow-up is needed. 
P3-540 Endocrine Consequences of Cancer

\section{Thyroid Dysfunction after using 131|-Meta-Iodobenzylguanidine (131I-MIBG) in the Treatment of Neuroblastoma in Childhood}

H.M. van Santen*1; J. de Kraker*1; B. van Eck*1; T. Vulsma*1. Introduced by B.J. Otten

${ }^{1}$ Academic Medical Center, Amsterdam, The Netherlands

Thyroid dysfunction after therapeutic use of ${ }^{131}$ I-MIBG in children with neuroblastoma (NB) was seen. The incidence was, however, unknown. Objective: The specific uptake and storage of ${ }^{131}$ I-MIBG by neuro-ectodermal tissues makes it suitable for treatment in advanced stages of NB. A drawback of this treatment is that ${ }^{131} \mathrm{I}$ is cleaved off from the administered ${ }^{131} \mathrm{I}-\mathrm{MIBG}$ and taken up by the thyroid. The accumulation of ${ }^{131} \mathrm{I}$ by the thyroid is blocked by $100 \mathrm{mg}$ KI daily for fourteen days. Despite KI prophylaxis, thyroid damage in some patients was observed. Aside from possible clinical consequences, the risk of thyroid malignancies developing after a prolonged period of thyrotropin elevation (TE) after radiation has been suggested in literature. Also, an excessive risk of thyroid cancer in NB patients after external radiation has been described. The objective of this study was to describe the incidence of thyroid dysfunction in our population of ${ }^{131} \mathrm{I}-\mathrm{MIBG}$ treated patients. Patients and Methods: A retro-spective study was performed in 44 patients treated with ${ }^{131}$ I-MIBG as initial therapy during childhood. Thyroid Stimulating Hormone (TSH) levels were screened as a reflection of thyroid function. TE was defined as TSH $>4.5$ $\mathrm{mU} / 1$. Results: 22 patients $(50 \%)$ presented TE, which was transient in 4 . The average number of treatments with ${ }^{131} \mathrm{I}-\mathrm{M}$ IBG was 3.3 per patient. The average follow-up time of 27 long term survivors was 3.5 years (range 6 mo- 8 years). 16 of these 27 patients $(60 \%)$ developed TE with FT4 values still within the normal range. No changes were seen on ultrasound. Clinical signs of hypothyroidism were not observed, however growth retardation was detected in 10 of 23 patients. In total 7 patients received suppletion therapy with thyroxine. No correlation was found between TE and thyroidal uptake of ${ }^{131} \mathrm{I}^{-}$, the number of treatments received or inefficient KI prophylaxis. Conclusions: The incidence of TE after treatment with ${ }^{131} \mathrm{I}-\mathrm{MIBG}$ for NB in children is high, in spite of KI prophylaxis. Close follow-up of thyroid function and growth is required in these patients. A prospective study with a new prophylactic approach for thyroid protection during treatment with ${ }^{131}$ I-MIBG is being performed.

P3-541 Endocrine Consequences of Cancer

\section{Endocrinopathies Prior to Radiation Therapy for} Childhood Brain Tumors

S.R. Rose ${ }^{* 1}$; T.E. Merchant ${ }^{* 2}$; J.M. Smith*2; T. Williams*2; L.E. Kun*2; R.K. Danish*1; G.A. Burghen*1;R.H. Lustig*1; Introduced by $M$. Maes

${ }^{1}$ University of Tennessee, Memphis, United States; ${ }^{2}$ St Jude

Children's Hospital, Memphis, TN, United States

Children with localized primary CNS tumors were prospectively evaluated for endocrinopathy prior to radiation therapy. Tumor diagnoses included ependymoma $(n=21)$, low-grade astrocytoma (17), high-grade astrocytoma (6), craniopharyngioma (5), and germinoma (1). There were 22 infratentorial tumors. Among the supratentorial tumors $(n=28), 23$ were centrally located in the suprasellar region $(\mathrm{n}=6)$, hypothalamus (3), thalamus (6), and optic pathway (8). Methods: Fifty children (26 girls), ages 2-18 years, were admitted to the hospital for ATT, L dopa test, TSH surge, TRH, $1 \mu \mathrm{g}$ ACTH, metyrapone, and GnRH tests. X-ray of the left hand and wrist was obtained to determine bone age. Results: Thirty-five (70\%) had evidence of endocrinopathy prior to radiation therapy including $55 \%(12 / 22)$ of patients with posterior fossa tumors, $80 \%(4 / 6)$ with non-central supratentorial tumors, and $83 \%(19 / 23)$ with central supratentorial tumors. Conclusions: The incidence of endocrinopathy prior to radiation therapy in pediatric brain tumor patients is high including those with tumors not located in or adjacent to the hypothalamic-pituitary unit. These data reveal that the actual rate of radiation-induced endocrinopathy has been overestimated. The potential for radiation-induced endocrinopathy alone cannot be used as an argument for alternatives to radiation therapy for most patients. Baseline endocrine function should be determined for brain tumor patients enrolled in prospective trials assessing the benefit and side effects of advanced radiation delivery techniques. Patients will benefit from such early identification of endocrinopathy and initiation of therapy. Pre-irradiation endocrinopathy may be an early indicator of CNS damage that is likely to influence functional outcome unrelated to radiation therapy.
P3-542 Endocrine Consequences of Cancer

Unusual Cause of Complete Hypopituitarism

H. Jung ${ }^{1}$; C. Schneekloth ${ }^{* 1}$; M. Tatagiba*1; F. Brassel ${ }^{* 1}$;

J.H.H.Ehrich*1

${ }^{1}$ Medical School Hannover, Hannover, Germany

Growth failure is a common indicator of impaired pituitary function in children We report a female patient, 16 years of age, presenting with short stature, decreased height velocity and delayed puberty but without visual complaints. Hormonal analysis revealed growth hormone deficiency, impaired pituitary-gonadal axis, secondary hypothyroidism and hypocortisolism but no central diabetes insipidus. Magnetic resonance imaging detected a $2.5 \times 2.5 \times 3.0$ intra- and suprasellar tumor shifting the optic chiasm ventrally. The lesion showed inhomogeneous enhancement of contrast medium in MRI, while computed tomography revealed calcifications. These characteristics were consistent with the diagnosis of a craniopharyngeoma. On transcranial surgery the "craniopharyngioma' was found to be an aneurysmatic bone cyst. Neither residual pituitary tissue nor the pituitary stalk were seen. Because of bone-like structure and the intense bleeding complications, requiring several blood transfusions, only partial removal of the cystic process was performed. Postoperative arteriography confirmed an aneurysmatic bone cyst originating from the left carotid artery eroding the clivus and dorsum sellae. After partial surgical resection no recovery of impaired pituitary function was seen. In contrast, the patient developed central diabetes insipidus. Therefore complete substitution therapy of pituitary hormones was necessary. Endovascular embolisation of this malformation was performed twice without improvement of pituitary function. In Conclusion: An aneurysmatic bone cyst can mimick craniopharyngioma due to the appearance of calcifications. To our knowledge, this vascular malformation is a very rare condition inducing pituitary insufficiency. However, differential diagnosis should include such a rare pituitary tumor in order to avoid unexpected surgical complications.

P3-543 Endocrine Consequences of Cancer

\section{Nocturnal Leptin Secretion in Children after Cranial Irradiation for Medulloblastomas}

M. Marx*1; J. Dötsch*1; J.D. Beck*1;W.F. Blum² ;

G.G. Grabenbauer ${ }^{* 3} ;$ W. Rascher*1; H.G. Dörr ${ }^{1}$

1 University Hospital for Children and Adolescents, Erlangen,

Germany; ${ }^{2}$ University of Giessen, Giessen, Germany; ${ }^{3}$ University of

Erlangen, Erlangen, Germany

Objective: (1) To assess the impact of cranial irradiation on nocturnal leptin secretion profiles of children. (2) To assess the influence of idiopathic growth hormone deficiency on nocturnal leptin secretion. Patients and Methods: (values given as median [range]): Analysis of 10-hour spontaneous nocturnal leptin secretion profiles (blood sampling: 8:00 pm to 6:00 am, intervals: $20 \mathrm{~min}$ ) in six medulloblastoma patients (MED: $3 \mathrm{~m}, 3 \mathrm{f}$, Tanner stage 1, growth hormone deficiency: $\mathrm{n}=5$, neurosecretory dysfunction: $\mathrm{n}=1$ )) after cranial radiotherapy (doses to whole brain/hypothalamus: 35.3 [32.0-43.3] Gy, to posterior fossa: 54.0 [48.0-56.6] Gy; posttherapeutic interval: 3.21 [1.33-5.08] years) and in 12 children with idiopathic growth hormone deficiency (IGHD: $9 \mathrm{~m}, 3 \mathrm{f}$, Tanner stage 1). Control: 12 children with normal variant short stature (NVSS: $7 \mathrm{~m}, 5 \mathrm{f}$, Tanner stage 1). Body mass index (BMI) and chronologic age did not differ between patients (MED, IGHD) and the control group. Results: (values given as median [range]): MED patients showed a significantly higher mean nocturnal leptin concentration than NVSS (8.96 [3.04-22.4] vs. $1.63[0.65-10.12] \mathrm{ng} / \mathrm{ml}, \mathrm{p}<0.05)$, in 4/6 MED patients absolute values were above the 99 th BMI-related percentile. This difference was not observed comparing IGHD patients with NVSS. Mean nocturnal leptin concentration showed a positive correlation with BMI in NVSS $(r=0.715, p<0.01)$ and IGHD patients $(r=0.998, p<0.001)$, but not in MED patients. For qualitative analyses, the profiles were calculated as mean percent change of the 10-hour mean of leptin concentration. In NVSS and IGHD patients a significant decrease of mean leptin concentration was found towards the early morning hours; profiles did not differ between these groups. Profiles of MED patients differed significantly from those of NVSS showing no drop of leptin concentration in the morning hours. Conclusions: (1) In children after cranial irradiation for medulloblastomas the physiological circadian rhythm of leptin secretion seems to be disturbed. From our results it cannot be decided whether this is due to direct hypothalamic injury resulting in a leptin insensitivity or to an alteration of secretory mechanisms for leptin. (2) Children with IGHD showed no alterations in nocturnal leptin secretion. Thus changes in medulloblastoma patients cannot be ascribed to disturbances in growth hormone secretion. 
P3-544 Endocrine Consequences of Cancer

Familial Medullary Thyroid Carcinoma with the V804M Mutation in the Proto-Oncogene RET: Findings in One Family

C. Pavia ${ }^{1} ;$ J. Oriola*2; L. Kasprzak*3; C. Villabona*4; S. Nolet*3

W. Foulkes*3; F. Rivera-Fillat*2

${ }^{1}$ Hospital Sant Joan de Déu, Barcelona, Spain; ${ }^{2}$ Laboratori

Hormonal, Hospital Clinic, Barcelona, Spain; ${ }^{3}$ Royal Victoria

Hospital, Montreal, Canada; ${ }^{4}$ Barcelona, Spain

Medullary thyroid carcinoma (MTC) may occur in a sporadic or a hereditary form as part of multiple endocrine neoplasia type 2 (MEN2). Familial MTC (FMTC) occurs in families in the absence of other manifestation. Germline mutations in the proto-oncogene RET are associated with MEN2 and FMTC and if presents, prophylactic thyroidectomy is indicated in carrier members. Family: From the case of a patient aged 43 (propositus), diagnosed of MTC, we carried out the study in his family: 2 daughters aged 5 years 2 months (case 1) and 1 year 3 months (case 2), and a son aged 3 years 6 months (case 3 ). In none of them a goiter existed nor by palpation nor by ultrasonography. In the paternal branch other MTC affected relatives were also studied: a cousin and his mother. In this family all affected members had prominent corneal nerves. Method: In the propositus, molecular study of the proto-oncogene RET was carried out by means of amplifying exons 10, 11, 13, 14, 15 and 16 and subsequent automatic sequencing. Pentagastrin stimulation test $(0.5 \mu \mathrm{g} / \mathrm{kg})$ was performed only in cases 1 and 2 . Results: Propositus case carried the relatively rare V804M mutation in exon 14. In addition, he also carried a V778I mutation in exon 13 . When family members were analysed, all affected members carried the V804M mutation. The V778I variant cosegregated with V804M mutation indicating both mutations were in the same allele. Case 2 was found to be a carrier for both mutations and thyroidectomy was performed. No malignant histopathology was observed. Basal plasmatic calcitonin concentration was $6.1,15$ and $13 \mathrm{pg} / \mathrm{ml}$ in cases 1,2 and 3 respectively. The post-stimulus values in cases 1 and 2 were 8.5 and $23 \mathrm{pg} / \mathrm{ml}$, respectively. Comment: The relatively rare mutation V804M has previously only found in FMTC families. The V778I variant perhaps could be contributing to the particular phenotype observed in this family.

P3-545 Endocrine Consequences of Cancer

\section{Hypothalamic-Pituitary Function and Leptin after} Cranial Irradiation in Childhood

L. Adan $^{* 1}$; J.C. Souberbielle*1; C. Kalifa*1; J.M. Zucker*1; G. Leverger*1; R. Brauner ${ }^{1}$

${ }^{1}$ Hôpital Necker-Enfants Malades, Paris, France

Patients given cranial irradiation (Rx) during childhood frequently lack $\mathrm{GH}$ and need GH replacement to normalize their growth. The factors and markers indicating the severity of this deficiency in adulthood are debated. In addition, they are overweight. 85 young adults ( 49 males) having GH deficiency after Rx for disorders not involving the pituitary area were evaluated at $15.7 \pm 0.2$ years $(13.5-19)$, 1 month after the end of GH therapy. They were classified in groups 1, ALL (18 or $24 \mathrm{~Gy}) ; 2$, glioma and others (40-62 Gy); 3, retinoblastoma (23-52 Gy); 4, medulloblastoma (25-40 Gy).

\begin{tabular}{llllrlll}
\hline Group & $\mathrm{n}$ & BMI & $\begin{array}{l}\text { Insulin } \\
\mathrm{mU} / \mathrm{l}\end{array}$ & $\begin{array}{l}\text { Leptin } \\
\mathrm{ng} / \mathrm{ml}\end{array}$ & $\begin{array}{l}\mathrm{GH} \\
\mathrm{ng} / \mathrm{ml}\end{array}$ & $\begin{array}{l}\text { IGF-I } \\
\mathrm{U} / \mathrm{ml}\end{array}$ & $\begin{array}{l}\mathrm{BP}-3 \\
\mathrm{mg} / \mathrm{l}\end{array}$ \\
\hline 1 & 28 & $0.6 \pm 0.2$ & $9.7 \pm 1.0$ & $14 \pm 2$ & $4.8 \pm 0.5$ & $1.3 \pm 0.2^{\mathrm{c}}$ & $2.0 \pm 0.1$ \\
2 & 27 & $1.3 \pm 0.3^{\mathrm{a}}$ & $9.7 \pm 1.5$ & $12 \pm 3$ & $1.9 \pm 0.4^{\mathrm{b}}$ & $0.5 \pm 0.1$ & $1.7 \pm 0.1$ \\
3 & 10 & $0.2 \pm 0.3$ & $8.9 \pm 0.9$ & $16 \pm 4$ & $4.3 \pm 1.5$ & $0.7 \pm 0.2$ & $1.6 \pm 0.1$ \\
4 & 20 & $0.5 \pm 0.3$ & $7.6 \pm 0.7$ & $9 \pm 2$ & $3.5 \pm 0.6$ & $1.0 \pm 0.2$ & $2.2 \pm 0.2^{\mathrm{d}}$ \\
\hline
\end{tabular}

$\mathrm{p}<{ }^{\mathrm{a}} 0.05 ;{ }^{\mathrm{b}} 0.001 ;{ }^{\mathrm{c}} 0.02 ; \mathrm{d} 0.03$.

GH peak was correlated with IGF-I $(p=0.006)$ and BP-3 $(p=0.05)$ and all of them with the irradiation dose ( $\mathrm{p}=0.04$ for BP-3 and 0.0001 for the others). IGF-I was also correlated with BP-3 $(\mathrm{p}=0.0001)$. Insulin and leptin were correlated between them $(p=0.0003)$ and with BMI $(p=0.005$ and $p=0.0001)$. The other deficiencies were: TSH in 29, most of them in groups 2 and 3; partial or complete gonadotropin deficiency in 10, mostly in group 2 and following precocious puberty; ACTH in one, prolactin in 4 patients. Nine young men had increased FSH due to testicular irradiation $(n=4)$ or chemotherapy $(n=5)$. Conclusions: Residual GH secretion in this population depends upon the irradiation dose given in childhood. IGF-I and BP-3 also reflect this finding. Leptin and insulin only reflect the accumulation of adipose tissue suggesting that there is no resistance secondary to the irradiation.

\section{P3-546 Endocrine Consequences of Cancer}

Changes in Height and Weight after Bone Marrow Transplantation (BMT)

R. Brauner ${ }^{1}$; A.C. Couto-Silva*1 ${ }^{* 1}$ C. Trivin ${ }^{* 1} ;$ H. Esperou ${ }^{* 1}$; J. Michon*1;A. Fischer*1

${ }^{1}$ Hôpital Necker-Enfants Malades, Paris, France

Short stature can be a severe side effect of BMT. This study analyses the changes in height and body mass index (BMI) of 53 patients given BMT. Group $1(\mathrm{n}=22)$ was given $12 \mathrm{~Gy}$ total body irradiation $(\mathrm{TBI})$ as 6 fractions, group 2 $(\mathrm{n}=14) 10 \mathrm{~Gy}$ (one dose) TBI, group $3(\mathrm{n}=8) 6 \mathrm{~Gy}$ (one dose) total lymphoid irradiation, and group $4(n=9)$ chemotherapy alone. At the first evaluation, $13 / 36$ patients in groups 1 and 2 had low growth hormone $(\mathrm{GH})$ peaks after stimulation. The mean \pm SE plasma insulin-like growth factor I (IGF I) concentrations (zs) were similar in groups $1(-2.9 \pm 0.3)$ and $2(-2.5 \pm 0.3)$, and in groups $3(-1.4 \pm 0.3)$ and $4(-1.4 \pm 0.7)$. The GH peaks and plasma IGF I concentrations did not correlate with each other, nor with height changes since BMT. After TBI, the BMI and leptin concentrations were correlated positively with each other $(p=0.005)$, and negatively with the GH peak $(p=0.02$ for BMI and 0.007 for leptin). In the 13 patients given $\mathrm{GH}$ therapy for more than 1 year, growth rate during the first year increased from $4.1 \pm 0.4$ to $6.1 \pm 0.5 \mathrm{~cm}(\mathrm{p}=$ $0.02)$. Adult height $(-1.2 \pm 1.0 \mathrm{SD}, \mathrm{n}=15)$ was lower than height at BMT $(-0.3$ \pm 1.4 SD) and than target height $(0.2 \pm 0.9 \mathrm{SD}, \mathrm{p}<0.01)$. Conclusion: This study shows that TBI actually decreases the GH secretion and interferes with the leptin secretion, but the markers of the GH secretion in a given patient and the indications of $\mathrm{GH}$ treatment remain difficult to establish.

P3-547 Endocrine Consequences of Cancer

Gonadal Function after Bone Marrow Transplantation (BMT) during Childhood

A.C. Couto-Silva*1; C. Trivin ${ }^{* 1} ;$ E. Thibaud ${ }^{* 1} ;$ H. Esperou ${ }^{* 1}$;

J. Michon $^{* 1} ;$ R. Brauner ${ }^{1}$

1 Hôpital Necker-Enfants Malades, Paris, France

Conditioning for BMT may alter the viability of the germ cells and the production of gonadal hormones. We analyzed the factors of the gonadal risk (gender and age, type of conditioning) in 66 patients given BMT for leukemia $(\mathrm{n}=32)$, aplastic anemia $(n=21)$, neuroblastoma $(n=8)$ or lymphoma $(n=5)$. They were divided in: Group 1 given 12 Grays (Gy) total body irradiation (TBI) as 6 fractions, Group $210 \mathrm{~Gy}$ (one dose) TBI, Group $36 \mathrm{~Gy}$ (one dose) total lymphoid irradiation (TAI) and Group 4 chemotherapy alone. 26 females $(84 \%)$ and 32 males $(91 \%)$ were in pre-pubertal age $(<10$ years in females and $<12$ years in males) at BMT.

\begin{tabular}{lllllll}
\hline Groups & $\mathrm{n}$ & $\begin{array}{l}\text { Age } \\
\text { years }\end{array}$ & $\begin{array}{l}\text { FSH } \\
(\mathrm{U} / \mathrm{l})\end{array}$ & $\begin{array}{l}\text { LH } \\
(\mathrm{U} / \mathrm{l})\end{array}$ & $\begin{array}{l}\text { Testo } \\
(\mathrm{ng} / \mathrm{ml})\end{array}$ & $\begin{array}{l}\text { Test vol } \\
(\mathrm{ml})\end{array}$ \\
\hline Males & & & & & & \\
1 TBI 12 & 9 & $15.4 \pm 0.9$ & $19 \pm 6$ & $5.4 \pm 2$ & $2.5 \pm 0.6$ & $5.6 \pm 0.6$ \\
2 TBI 10 & 14 & $14.2 \pm 0.5$ & $37 \pm 11$ & $8.9 \pm 3$ & $2.4 \pm 0.6$ & $4.4 \pm 0.5^{*}$ \\
3 TAI & 9 & $15.9 \pm 0.5$ & $25 \pm 11$ & $6.4 \pm 3$ & $3.4 \pm 0.4$ & $8.2 \pm 1.0$ \\
4 CHEMO & 3 & $14.4 \pm 2$ & $3.8 \pm 2$ & $1.6 \pm 0.4$ & $1.8 \pm 1.7$ & $7.8 \pm 2.0$ \\
\hline Females & & & & & & \\
1 TBI 12 & 13 & $13.3 \pm 0.6$ & $65 \pm 12$ & $22.4 \pm 6$ & & \\
2 TBI 10 & 5 & $16 \pm 0.5$ & $52 \pm 23$ & $24 \pm 9.5$ & & \\
3 TAI & 7 & $14.8 \pm 06$ & $47 \pm 15$ & $10 \pm 3$ & & \\
4 CHEMO & 6 & $11.8 \pm 05$ & $21 \pm 13$ & $4 \pm 3$ & & \\
\hline
\end{tabular}

At the last evaluation: mean $\pm \mathrm{SE} ; * \mathrm{p}<0.05$ compared to group 3

Spontaneous menarche occurred in $52 \%$ of the females aged $>15$ years. Associated endocrine abnormalities were GH deficiency (35\%) in groups 1 and 2 and hypothyroidism $(42 \%)$ in groups $1-3$. There was no correlation between FSH and testicular volume, LH and testosterone. Conclusion: The germ cells of the testis are frequently altered particularly by irradiation, but the secretion of testosterone is preserved in many patients. Patients conditioned with chemotherapy that is toxic for the gonads, or those who had gonadal irradiation are seen for evaluation at pubertal age. 
P3-548 Endocrine Consequences of Cancer

Endocrine Disorders in Children with Neurofibromatosis Type 1 (NF1) and Optic Glioma A. Pierre-Kahn ${ }^{* 1} ;$ R. Brauner

${ }^{1}$ Hôpital Necker-Enfants Malades, Paris, France

Optic glioma (OG) occurred in 15\% of children with NF1 (Listemick, J Pediatr 1994). It may alter the hypothalamic-pituitary (HP) function directly and/or by the cranial irradiation $(\mathrm{Rx})$ used in its treatment. 20 patients with NF1 and OG were evaluated before $(n=11)$ and after $\mathrm{Rx}(45-55 \mathrm{~Gy})$ to determine the respective effect of $\mathrm{OG}$ and $\mathrm{Rx}$ on $\mathrm{HP}$ function. The $\mathrm{NFl}$ was diagnosed before the $\mathrm{OG}$ in 14 of them. For the whole population, the OG was revealed by ophthalmologic signs $(n=16)$, central precocious puberty $(C P P, n=2)$ or diagnosed on systematic neuroradiological evaluation in $\mathrm{NFl}(\mathrm{n}=2)$. Before $\mathrm{Rx}, 2$ had CPP, 1 had GH deficiency and 4 had both. After Rx, 2 additional CPP occurred. For the whole group followed 3.4-21.3 (10.9) years after Rx, the deficiencies occurred in $100 \%$ of cases for $\mathrm{GH}$, in $65 \%$ for TSH, in $35 \%$ for gonadotropins and in $1 \%$ for ACTH. Substitutive treatments were given, particularly $\mathrm{GH}$ in 18 and $\mathrm{GnRH}$ analogs in 12 patients, in association in 11. Adult height was reached in 14 cases. It was at -1.9 ( -4.5 to 1$) \mathrm{SD}$, lower than target height $(-0.4$ SD). Conclusion: The endocrine manifestation of OG is CPP, and less frequently $\mathrm{GH}$ deficiency in more invasive tumors. The Rx induces $\mathrm{GH}$ deficiency in all cases. The association of $\mathrm{GH}$ and $\mathrm{GnRH}$ analog treatments preserves the growth potential. Endocrine sequelae will probably change as chemotherapy is preferred to radiotherapy partly because of the occurrence of cerebral vasculopathy.
F. Monceaux ${ }^{* 1}$; J.C. Souberbielle*1; C. Kalifa*1; J.M. Zucker*1;

P2-550 Neuroendocrinology

Neurochemical Identity of Androgen

Receptor-Containing Neurons in the Arcuate Nucleus in the Adult Male Rat

M. Fodor*1; C.B.M. Oudejans*1; H.A. Delemarre-Van de Waal ${ }^{1}$

${ }^{1}$ University Hospital 'Vrije Universiteit', Amsterdam,

The Netherlands

Testosterone exerts important feedback effects on the hypothalamus to influence the gonadotropic and somatotropic axes in the adult male rat. Several studies have demonstrated that neurons within the arcuate nucleus respond to castration by increased and/or decreased mRNA levels of tyrosine hydroxylase $(\mathrm{TH})$, growth hormone-releasing hormone (GHRH), neuropeptide Y (NPY) and proopiomelanocortin (POMC)-related peptides. The altered mRNA concentration can be prevented completely or partly by the administration of testosterone or a nonaromatizable androgen (i.e., dihydrotestosterone). While these observations suggest that androgen receptors mediate the actions of gonadal steroids on arcuate neurons, they do not provide any information about the location of the androgen receptors involved in this process. To determine whether these neurons themselves express androgen receptors, we double immunolabeled hypothalamic sections from colchicine-pretreated male rats $(\mathrm{n}=5)$ using polyclonal antiserum to androgen receptor (PG21) and polyclonal antisera to either TH, GHRH, NPY or $\alpha \mathrm{MSH}$. Androgen receptor immunopositivity was detected in the nucleus of neurons throughout the hypothalamus. Double labeled immunohistochemistry revealed the presence of androgen receptor in only $2 \%$ of $\alpha \mathrm{MSH}$ neurons. Although, there was an overlap in the anatomical distribution of GHRH, NPY, TH and androgen receptor-containing cell bodies, none of the GHRH, NPY or TH immunolabeled cells we examined in each hypothalamus appeared to contain androgen receptors. The absence or very small population of double labeled neuroendocrine cells suggest that these neurons are not direct targets for androgens and therefore the effects of testosterone must be produced indirectly by some other neural or endocrine intermediary process.

P2-551 Neuroendocrinology

P2-549 Neuroendocrinology

The Effect of Cranial Irradiation for Tumours Distal to Pituitary on ACTH Secretion

H.A. Spoudeas ${ }^{1}$; E. Charmandari*1; C. Brook ${ }^{1}$

${ }^{1}$ University College London, London, United Kingdom

Background: Cranial irradiation is the main hope of cure for children with brain tumours (BT), but this is increasingly being avoided because it is blamed for long-term neuro-endocrine and cognitive morbidity. $\mathrm{GH}$ insufficiency results in the majority of patients treated for non-central BT but the prevalence of other anterior pituitary deficits is less clear. Adult studies have suggested a high prevalence of ACTH insufficiency but these involved pituitary tumours. Aims: To investigate the effect of cranial irradiation for non-central brain tumours on anterior pituitary secretion in adult survivors of childhood BT. Methods: We studied HPA integrity in 20 children (14 males, 6 females) who presented with posterior fossa tumours at $2.5-13.3$ years (median: 6.2 years). Median radiation doses to posterior fossa and hypothalamic-pituitary area were 50 and $40 \mathrm{~Gy}$, respectively. HPA function assessment was undertaken twice, at $0.5-2.5$ years (median: 1.4 years) and $4.4-14.6$ years (median: 9.0 ) after radiotherapy. Duration of follow up ranged from 4.5 to 21 years (median: 10.2 years). Results: All patients mounted an inadequate $\mathrm{GH}$ response to insulin-induced hypoglycaemia both at initial assessment (at growth failure) and at completion of growth and puberty. Cortisol response at first and last follow-up was appropriate (peak $>500 \mathrm{nmol} / 1$ with $>200 \mathrm{nmol} / 1$ increment) in $18(90 \%)$ patients; but two achieved only $\geq 350 \mathrm{nmol} / 1$. These last, however, had normal responses to low dose $\left(500 \mathrm{ng} / 1.73 \mathrm{~m}^{2}\right)$ ACTH stimulation (peak $>500 \mathrm{nmol} / 1$ ) and none required glucocorticoid substitution. During follow up an evolving anterior pituitary endocrinopathy was detected in only one patient $(5 \%)$ who developed secondary hypothyroidism. However, a further four $(20 \%)$ and 2 $(10 \%)$ had primary hypothyroidism and gonadal dysfunction, respectively, evidenced as a raised pituitary message. Conclusions: ACTH insufficiency is rare after $40 \mathrm{~Gy}$ pituitary irradiation. The previous reported high prevalence is most likely due to evolving neuronal damage resulting from tumour or previous surgery for pituitary masses. GH remains peculiarly sensitive and the deficit is permanent. Thyroid and gonadal dysfunction are much less common, and usually result from primary glandular toxicity. Cranial irradiation is not the only culprit in neuro-endocrine morbidity; this observation is important in determining future potentially curative treatments for children with BT.

39th Annual Meeting of the ESPE Macroprolactinoma in Childhood

K.P. Ullrich ${ }^{* 1} ;$ K. Gerlach ${ }^{* 2} ;$ E. von Büren ${ }^{* 3} ;$ K. Klemp ${ }^{* 1} ;$ Introduced by W. von Petrykowski

${ }^{1}$ Children's Hospital Helios Clinic Gotha, Gotha, Germany;

${ }^{2}$ Research Center of Medical Technology, Erfurt, Germany;

${ }^{3}$ Schwarz Pharma AG, Monheim, Germany

Macroprolactinoma in childhood are very seldom. We report on a 10-year treatyears. Prior to treatment the size of the tumor was $2 \times 2 \times 2 \mathrm{~cm}$. Several hypophyseal bleedings occurred after start of bromocriptin therapy leading to secretion deficiency of growth hormone and of gonadotropins. During the following 6 months the size of the tumor decreased to $1 \times 1 \times 1 \mathrm{~cm}$. After switching medication to quinagolid the serum prolactin values decreased to normal and the size of the tumor has been remaining stable at $3 \times 3 \times 3 \mathrm{~mm}$ for the last 4 years (fig. 1). The binasal visus impairment receded completely. The conservative long-term treatment of a prolactinoma in childhood is an appropriate alternative to surgical intervention.

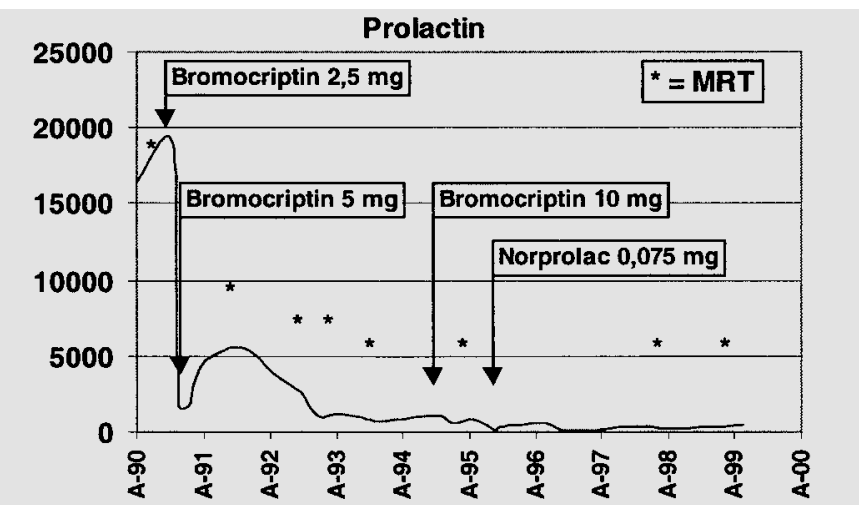

Fig. 1. ment with bromocriptin and quinagolid (Norprolac) in a girl with an age of 21 
P2-552 Neuroendocrinology

Overnight Profiles of Melatonin, LH, FSH, GH and Prolactin Combined with Polysomnography in Children and Adolescents

K.P. Ullrich ${ }^{* 1}$; K. Klemp*1;A. Hoffmann*2; M. Suhr*2;

E. von Büren ${ }^{* 3}$; S. Nikolaus ${ }^{* 4} ;$ K. Gerlach ${ }^{* 2}$; Introduced by W. von Petrykowski

${ }^{1}$ Children's Hospital Helios Clinic Gotha, Gotha, Germany;

${ }^{2}$ Research Center of Medical Technology, Erfurt, Germany;

${ }^{3}$ Schwarz Pharma AG, Monheim, Germany; ${ }^{4}$ Ferring Arzneimittel

$\mathrm{GmbH}$, Kiel, Germany

Aim: To detect relationships between overnight profiles of melatonin (MLT), LH, FSH, GH, prolactin and polysomnography. Methods: Blood sampling was taken every 30 minutes from 20.00 to 08.00 using the Con-flow-pump (Swemed). Polysomnography was recorded with Alice III. Analysis of hormones was performed with Pulsar (Wachter) and Ancopuls (Albers). Patients: 29 children aged 7-18:17 with growth hormone deficiency, 12 without hormonal deficiencies, and 3 had hypothalamic disorders. Results: In the polysomnography of all children (pre- and pubertal) MLT-profiles were found to relate to the periods of sleep onset and awake time. A positive correlation of MLT and GH was found at onset of sleep and prior to wake up. In the 2 nd half of the sleep MLT and GH dissociate. No correlation was found between GH and sleep phase. Low hormonal secretion does not appear to influence sleep architecture and MLT secretion in hypothalamic disorders.

P2-553 Neuroendocrinology

The Response of Variant (v) and Wild Type (wt)

Luteinizing Hormone (LH) Secretion to GnRH

Stimulation in Heterozygous Subjects

H. Virtanen ${ }^{* 1} ;$ C. Nilsson ${ }^{* 1} ;$ M. Kaleva*1; A. - M. Haavisto ${ }^{* 1}$;

K.Pettersson ${ }^{* 1}$; J. Toppari*1; I. Huhtaniemi*1; Introduced by

N.E. Skakkebæk

1 University of Turku, Turku, Finland

A variant form of $\mathrm{LH}$ occurs with high frequency in the Finnish population (28\% homo- or heterozygotes). v-LH has two point mutations in the LH $\beta$ subunit gene, both changing amino acid (Trp8Arg and Ile15Thr). v-LH has been shown to have increased in vitro bioactivity and a decreased half-time in vivo, and the v-LH $\beta$ promoter is more active than that of the wt allele. However, the molar proportions of secretion of these two $\mathrm{LH}$ forms are not known either basally or in response to GnRH stimulation. Objective: We wanted to evaluate whether the relative proportions of v- and wt-LH alter after GnRH stimulation, as a sign of differences in their intracellular processing and secretion. Subjects and Methods: A GnRH stimulation test was performed on 10 males aged 19-29 years and 7 females aged 18-23 years, known to be LHb heterozygotes. In the females, GnRH test was performed in late follicular phase, between days 9-11 of cycle. All females had had at least one normal menstrual cycle if they had previously used contraceptive pills. None of the subjects had medication known to affect the function of hypothalamus-pituitary-gonadal axis. A catheter was placed in a forearm vein and 4-ml samples of blood were taken $10 \mathrm{~min}$ and immediately before, and 10,20,40,60,120, and $180 \mathrm{~min}$ after an iv injection of $50 \mu \mathrm{g} \mathrm{GnRH}$ (Relefact ${ }^{\circledR}$, Hoechst). Serum wt and total LH concentrations were determined using two immunofluorometric assays (IFMA), one recognizing only wt-LH, the other recognizing both forms equally. All samples from an individual subject were run in duplicate in the same assay. The relationship between v- and wt-LH secretion was assessed by calculating the ratios of LH measured by the two IFMAs. One-way analysis of variance, followed by Tukey's multiple comparison test, was used for statistical analysis. Results and Conclusions: In male vLH heterozygotes, the proportion of wt-LH increased significantly at 40 (mean 5.7\%, p < 0.05), 60 (mean $6.0 \%, \mathrm{p}<0.01$ ), and 120 (mean $6.2 \%, \mathrm{p}<0.01$ ) min after GnRH injection, in keeping with the known shorter half-life of v-LH. In females, no significant differences were observed in the proportions after GnRH stimulation. These findings suggest that there are sex differences in the intracellular processing and secretion between $\mathrm{v}$ - and wt-LH.
P2-554 Neuroendocrinology

The Spectrum of Optic Nerve Hypoplasia-Septo-Optic Dysplasia: Clinical, Hormonal and Radiological Characterisation

P.E. Clayton 1 ; S. Sinha*1; N. Wright*2; D.A. Price 1; I.C. Lloyd*3

${ }^{1}$ University of Manchester, ${ }^{2}$ Royal Manchester Children's Hospital,

${ }^{3}$ Royal Eye Hospital, Manchester, United Kingdom

Septo-optic dysplasia (SOD) describes the association of optic nerve hypoplasia $(\mathrm{ONH})$, agenesis of midline structures (particularly the septum pellucidum and corpus callosum) and hypopituitarism. A spectrum of abnormality is recognised, from ONH as the only entity to SOD with panhypopituitarism. SOD has been considered a sporadic condition. However, a homozygous null mutation in the pituitary development gene HESX-1 in two siblings with SOD has been reported. The diverse range of abnormality in this condition implies that a number of candidate genes may be involved. We have therefore characterised all patients $(n=54$, male 31, female 23) presenting to ophthalmology and/or endocrine services with a clinical diagnosis of $\mathrm{ONH}$. The age at presentation ranged from birth to 11.3 years with eye signs (nystagmus, poor vision, strabismus) being the commonest presentation. $10(19 \%)$ had a primary endocrine presentation. On the basis of MR/CT scan reports $(\mathrm{n}=40), 3$ groups were defined: (1) Clinical diagnosis of $\mathrm{ONH}$ but 'normal' scan, $\mathrm{n}=9$; (2) clinical ONH, confirmed on scan but no midline problem, $\mathrm{n}=12$, and (3) clinical and radiological ONH and midline abnormality, $\mathrm{n}=19$. The ages at presentation (mean, range) and sex ratios were: (1) 1.3, 0-9.6 years, 1.25 (2) 2.4, $0.1-7$ years, 3 and (3) 1.4, $0-10.1$ years, 0.9 . The prevalence of endocrine disturbance was $22 \%$ in group $1,67 \%$ in group 2 and $74 \%$ in group 3 . GH and gonadotropin $(\mathrm{Gn})$ deficiencies were the main abnormalities in group 2 , with panhypopituitarism $(26 \%)$, diabetes insipidus $(26 \%)$ and precocious puberty $(26 \%)$ the main problems in group 3 . The sizes of the optic nerves on sagittal MR views were assessed in 26, and compared to those in 18 control children with isolated GH deficiency or congenital hypopituitarism. Only 1 child in groups 2 and 3 and all the controls had an optic nerve height $1.9 \mathrm{~mm}$. We may conclude: (1) ONH-SOD covers a wide spectrum of disorder. (2) MR scan is vital in assessment, and criteria need to be applied to define ONH. (3) All patients should have endocrine evaluation. (4) Those with ONH alone appear to be distinct from those with SOD - older at presentation, more likely to be male and to have $\mathrm{GH}$ and $\mathrm{Gn}$ deficiencies but not diabetes insipidus. This might imply differences in aetiology.

P2-555 Neuroendocrinology

Parturition Triggers the Fetal Hypothalamic GnRH Pulse Generator

A.-S. Parent*1; M.-C. Lebrethon ${ }^{1} ;$ A. Gerard ${ }^{* 1} ;$ V. Matagne ${ }^{* 1}$; J.-P. Bourguignon ${ }^{1}$

1 University of Liège, Liège, Belgium

Using explants of the retrochiasmatic hypothalamus from male and female rats, pulsatile secretion of Gonadotropin-releasing hormone $(\mathrm{GnRH})$ can be observed from the first postnatal day and progressively increases in frequency between day 5 and day 25 , before onset of puberty. In late fetal life, spontaneous pulsatile $\mathrm{GnRH}$ secretion is not observed. This does not result from immaturity of GnRH neurons since intermittent incubation with N-methyl-D-aspartate causes GnRH release to mimic the spontaneous secretion from mature explants. Our aim was to investigate the factors accounting for the occurrence of $\mathrm{GnRH}$ pulsatility perinatally. We first considered the withdrawal from inhibitory placental factors. This became unlikely since explants obtained from rats born by cesarean section after the onset of labor did not show any pulsatile secretion, in contrast to explants obtained one hour after natural parturition. Thus, we hypothesized the role of endocrine events occurring during parturition and we studied the effects of oxytocin and prostaglandin E2 (PG E2). Using explants obtained in late fetal life (20-21 days of gestation) and incubated with oxytocin $\left(10^{-8} M\right)$ and PG E2 $\left(10^{-6} M\right)$, GnRH pulsatility occurred with an interpulse interval of $120 \mathrm{~min}$. Using explants from neonatal rats on day 1 , the mean (SD) $\mathrm{GnRH}$ interpulse interval $(88 \pm 4 \mathrm{~min})$ was significantly increased $(+25 \%)$ by $10^{-7} \mathrm{M}$ of an oxytocin antagonist (110 $\left.\pm 4 \mathrm{~min}\right)$. Using explants from female rats at day 15 and 50 , the GnRH interpulse interval $(59 \pm 5$ and $37 \pm 4$ min, respectively) was significantly reduced $(-17 \%$ and $-6 \%)$ by oxytocin $(49 \pm 4$ and $35 \pm 4 \mathrm{~min})$ and significantly increased $(+22 \%$ and $+15 \%)$ by the oxytocin antagonist (72 \pm 4 and $43 \pm 4 \mathrm{~min}$ ). In conclusion, the parturition-related factors oxytocin and PG E2 may initiate pulsatile GnRH secretion perinatally. Endogenous oxytocin may be further involved with a potency decreasing throughout postnatal development. 
P2-556 Neuroendocrinology

High Rate of Recurrence for Childhood Cushing's Disease Treated by Transphenoidal Surgery

L. Levitsky*1; B. Swearingen*2; Introduced by R.I. Hintz

${ }^{1}$ Mass. General Hospital for Children, Boston, MA, United States;

${ }^{2}$ Boston, MA, United States

The recurrence rate for Cushing's disease in adults successfully treated by transphenoidal surgery is low. A recurrence rate of $7 \%$ after a mean of 5.7 years was reported in 136 adult patients from this institution. In contrast, recurrence rate for childhood Cushing's was as high as $50 \%$ in one series in which $5 / 22$ patients had relapses within 6 months of initial treatment, and 7 relapsed during longterm follow-up. Objective: To examine the outcome of Cushing's disease in a childhood series treated successfully with transphenoidal surgery without early relapses. Patients and Methods: We carried out a retrospective review of 10 children with Cushing's disease managed at this institution since 1989 of whom 8 had more than 1 year follow-up. Mean age was $13.1 \pm 0.9$ years (range 7.316.4 ) at surgery and follow-up was $4.2 \pm 1.0$ years (range $0.1-10.3$ ). Diagnostic evaluations, immediate outcomes of surgery, residual endocrine deficits, and recurrences of hypercortisolism were documented. Results: Five patients had normal pituitary anatomy on MRI, 3/4 patients with MRI evidence of tumor had tumor localization consistent with MRI findings, 1 patient had a small anterior pituitary with an ectopic posterior pituitary. Six patients without MRI localization underwent petrosal sinus sampling. One required repeat sampling to define a pituitary ACTH source. In 5/6 patients, surgery confirmed lateralization. Post-surgery, diabetes insipidus was a transient finding in 7 . Three patients required a second surgery within 1 week because of persistent cortisol secretion. One has residual anterior hypopituitarism. Of the $8 / 10$ children followed for 1 year, 2 have had recurrent hypercortisolism (after 2.1, 3.2 years) requiring intervention, and 1 has borderline hypercortisolism after 7.0 years. Therefore the risk of recurrence after a mean follow-up of $5.2 \pm 1.0$ years (range 1.0-10.3) is 25-35\%. Conclusions: The risk of recurrent hypercortisolism in childhood Cushing's disease is increased compared with the adult risk even when the surgical procedure is not associated with short-term recurrences.

\section{P3-557 Neuroendocrinology}

\section{Cell Lines from Pediatric Sarcomas of Neuronal Origin} Express the Insulin-Receptor-Related Receptor (IRR) Highly

M.W. Elmlinger ${ }^{* 1} ;$ B.S. Schütt ${ }^{1} ;$ E. Koscielniak ${ }^{* 2} ;$ W. Kiess $^{3}$; M.B. Ranke

1 University Children's Hospital, Tübingen, Germany; ${ }^{2}$ Olga Hospital, Stuttgart, Germany; ${ }^{3}$ University Children's Hospital, Leipzig, Germany

Introduction: In respect to its structure and tyrosine kinase signaling the insulin-receptor-related receptor (IRR) is a typical member of the insulin-receptor family. In parallel to the nerve growth factor receptor trkA, IRR mRNA is primarily expressed in neuronal crest-derived sensory neurons during embryogenesis. However, so far no ligand and thus no function for the IRR is known. Methods: In order to elucidate its function, IRR mRNA expression (RTPCR) was compared in 18 pediatric tumor cell lines from neuronal (neuroblastoma, Ewing- and neurofibrosarcoma) with that in sarcoma of other origin. Since IGF-I-receptor (IGF-I-R) expression was found altered in some sarcoma, the IGF-I-R mRNA was measured in parallel. Results: First, high IRR mRNA, i.e. $10 \%$ of GAPDH mRNA expression (range: 11-67 units) was found in all 7 neuroblastoma and in 2 of the 5 sarcoma of neuronal origin. Second, a high correlation $(\mathrm{r}=0.82)$ of the level of IRR mRNA with IGF-I-R mRNA was found in tumor cells of neuronal origin. Conclusions: The tight coupling of IRR and IGF-I-R expression suggests a functional association with the IGFsignaling system. Since the IRR is also known to correlate with the nerve growth receptor trkA which is a proto-oncogen, a role for IRR and its prospective ligand in tumorigenesis and in differentiation of neuronal cells is likely.
P3-558 Neuroendocrinology

\section{Growth Hormone Secretory Pattern in Childhood} Pituitary Microadenoma

A. Grossi*1;P. Cambiaso*1;S. Benedetti*1; G.L. Natali*1;

G. Fariello*1; P. Borrelli 1 ; Introduced by P. Borrelli

${ }^{1}$ Bambino Gesu Pediatric Hospital, Rome, Italy

Pituitary adenomas occur infrequently in childhood and adolescence, representing less than $2 \%$ of all intracranial tumors in this age group, and less than $10 \%$ of all pituitary adenomas in most surgical series. Objective: To evaluate possible alterations of $\mathrm{GH}$ secretion in children with Magnetic Resonance Imaging (MRI)-demonstrated intrasellar pituitary adenomas. Patients and Methods: A group of 14 children ( 9 males, 5 females, age 7-15.8 years), with non-secreting (NSAs, 13 patients) and PRL-secreting (1 patient) adenomas was studied. Symptoms included short stature (10 patients), precocious puberty ( 2 patients), secondary adrenal insufficiency (1 patient), hypogonadotropic hypogonadism (1 patient). The following tests were performed: (A) Insulin (ITT); (B) Clonidine (CLO); (C) GHRH + Pyridostigmine (GHPD); (D) Arginine (ARG). Results: ITT GH peak $(5.59 \pm 3.27 \mathrm{ng} / \mathrm{ml})$ and AUC $(403 \pm$ 187) were significantly lower than CLON $(10.8 \pm 3.55$ and $696 \pm 187, \mathrm{p}<$ $0.001)$. ARG GH peak $(6 \pm 4.74 \mathrm{ng} / \mathrm{ml})$ was significantly lower than CLON $(\mathrm{p}<0.05)$. No significant difference was found in both $\mathrm{GH}$ peak and AUC between ARG and ITT. GHPD showed significantly higher GH peak and AUC compared to ITT, CLON and ARG. Conclusions: These data show a reduction of GH response to ITT and to ARG, suggesting: a) ITT is a sensitive test for $\mathrm{GH}$ secretion and it seems to be altered before other usually performed GH stimulation tests; b) a low ITT-induced (and ARG-induced) GH could allow to hypothesize the presence of pituitary NSAs; c) for this reason an MRI examination has always to be performed before any GH replacement therapy; d) the lower ITT (and ARG)-induced GH response suggests alterations at hypothalamic rather than pituitary level in these patients.

\section{P3-559 Neuroendocrinology \\ Hormones May Predict Neurones: A Clinical and Genetic Study on the Cause and Course of Septo-Optic-Dysplasia \\ H. Krude ${ }^{1}$; N. Haufs ${ }^{2}$; H. Frisch ${ }^{3}$; S. Schwarz ${ }^{* 4}$; A. Grüters ${ }^{2}$ ${ }^{1}$ Charite-Children's Hospital, Germany; ${ }^{2}$ Berlin, Germany; \\ ${ }^{3}$ Vienna, Austria; ${ }^{4}$ Innsbruck, Austria}

Septo-optic-dysplasia (SOD) is one of the most variable endocrine diseases due to the extent of hypothalamic-pituitary malformations in association with different CNS abnormalities like absence of septum pellucidum and hypoplasia of the optic nerves. The clinical course of 20 nonrelated patients with SOD was studied propectively and a significant correlation of unfavourable mental and neurological outcome was found to the severity of the endocrine defects although hormone replacement therapy was started early and was closely monitored. Therefore the degree of the endocrine defect seems to reflect the severity of the developmental defect of the CNS. In addition we studied in this cohort of SOD patients the coding region of the HESX1 gene which has been shown to be mutated in one familial case of SOD. Although for the first we could identify the genomic sequence of an additional alternatively spliced 5-prime exon we were unable to demonstrate gene variations. Therefore we consider alternative molecular mechanisms as the cause of SOD. 
P3-560 Neuroendocrinology

Two New Mutations in the Vasopressin-Neurophysin-II Gene Associated with Familial Neurohypophyseal Diabetes insipidus

S. Rittig ${ }^{1}$; C. Siggaard ${ }^{* 1}$; V. Hána*2; L. Kovacs ${ }^{* 3}$; Introduced by O. Wolthers

${ }^{1}$ Skejby University Hospital, Aarhus, Denmark; ${ }^{2}$ University Hospital of Prague, Prague, Czech Republic: ${ }^{3}$ Comenius University Medical School, Comenius, Slovakia

Familial neurohypophyseal diabetes insipidus (FNDI) is a rare autosomal dominant disorder due to a deficient pituitary secretion of arginine vasopressin (AVP) and characterized by chronic polyuria and polydipsia. The deficiency of AVP secretion develops during early childhood and there is evidence that the deficiency is caused by degeneration of magnocellular neurons in the supraoptic nuclei. Since 1991 the disease has been linked to more than 30 different mutations in the gene encoding the vasopressin-neurophysin II protein. The exact mechanism by which the mutations cause the disease is still unclear. Purpose: To investigate the molecular genetic background in two kindreds (Ho and $\mathrm{Co}$ ) with clinical evidence of FNDI. Methods: A sequencing method was used for sequenase dye-terminator sequencing directly on single-stranded PCR-amplified genomic DNA obtained from a blood sample. The sequencing results were confirmed by restriction enzyme digestion analysis. Material: In family Ho, 3 affected individuals from three generations were analyzed and in family $\mathrm{Co}, 4$ affected from four generations were studied. All affected subjects had severe polyuria and polydipsia since childhood. Results: The results showed that the affected subjects in each of the 2 families had a new and so far unreported single base mutation in the coding region of the AVP-NPII gene. In the Co family, the 4 studied affected members all had a mutation in exon 2 (T1907 $\rightarrow$ G, predicting Cys73 $\rightarrow$ Gly) located in the highly preserved part of NPII. This mutation, which eliminates an important disulphide bridge in the carboxyterminal end of the hormone precursor protein, co-segregated perfectly with the disease phenotype in this family. The Ho kindred had a missense mutation in exon $1(\mathrm{G} 227 \rightarrow \mathrm{A}$, predicting a deletion of 4 amino terminal residues of the signal peptide, SP-19-16). This mutation is likely to severely interfere with proper targeting and cleavage of the signal peptide moiety. Conclusion: In summary, we have identified 2 new gene mutations that are likely to induce conformational changes of the three-dimensional NPII structure that could impair the normal intracellular processing of the pro-hormone. This is consistent with the hypothesis that FNDI is caused by a toxic effect of abnormally folded precursor protein.

\section{P3-561 Neuroendocrinology}

\section{Hypogonadotrophic Hypogonadism in an Obese Boy Having a Large Arachnoid Cyst (AC) of the Posterior Fossa}

C. Procopiuc $^{1}$; D. Hortopan 1 ; A. Caragheorgheopol ${ }^{1}$,

C. Dumitrescu ${ }^{1}$; M. Popa ${ }^{1}$

${ }^{1}$ Department of Pediatric Endocrinology, Institute of Endocrinology, Bucharest, Romania

Obesity and endocrine disturbances are usual in children bearing suprasellar and eventually anterior or middle cranial fossa ACs. Posterior fossa location seems to be devoid of any impairment of endocrine function. Case Report: A 14-year-old boy presented for overweight and very small genitalia. Medical history revealed a normal birth and birthweight and confused episodes of fainting at $1-3$ years. Physical examination revealed an obese (BMI $>30$ ) boy, a bit macroskelic and having a normal stature and a large head (circumference 60 $\mathrm{cm})$. The fat was female-type deposited. No axillary and pubic hair was noted. The testes were both $2 \mathrm{ml}$ and hard. The extended flaccid penis length was $<20$ $\mathrm{mm}$. No visceral or neurologic signs or syndromes were noted. Visual fields and FO were normal as well as cognitive skills. Bone age was 11 and the skull lateral roentgenography showed a double contoured floor of the sella turcica. CT scan showed a large arachnoid cyst of the posterior fossa with compression on the aqueduct of Sylvius and dilatation of 3rd and lateral ventricles. Secondary empty sella and a small, compressed pituitary were also noted. Results of Hormonal Investigations: Both FSH and LH were under $1 \mathrm{mU} / \mathrm{ml}$. TRH test showed a normal TSH response. ITT showed a normal but not brisk GH (peak $>15 \mu \mathrm{U} / \mathrm{ml}$ ) and a normal cortisol (peak $>30 \mu \mathrm{g} / \mathrm{dl}$ ) responses. Chlorpromazine test $(0.3 \mathrm{mg} / \mathrm{kg})$ revealed no prolactin response (peak $<15 \mathrm{ng} / \mathrm{ml}$ ) and a blunted response of GH (peak level 11.5). The hCG test resulted in a ten times increase of testosterone in serum. Conclusions: A large cyst of the posterior fossa associated with internal hydrocephalus and secondary empty sella is presumably linked to a hypothalamic dysfunction manifesting as obesity and hypogonadotrophic hypogonadism, probably irreversible. Though not described until now, it is conceptually easy to accept such association in many other cases of cysts of the posterior cranial fossa. So, such cysts should be carefully screened for endocrine disease, especially for hypothalamic dysfunction.

\section{P3-562 Neuroendocrinology \\ Endocrine Outcome in 67 Children after Radical Removal of Craniopharyngioma}

N. Mazerkina*1 $^{*}$;S. Gorelyshev*1; A. Tiulpakov ${ }^{2}$;E. Kuznetzova*2 ; V. Peterkova ${ }^{2}$

1 Moscow Neurosurgery Institute, Moscow, Russian Federation;

${ }^{2}$ Moscow Endocrinological Centre, Moscow, Russian Federation

We have examined endocrine function in 67 children ( 40 boys, 27 girls, age 3.8 to 17.9 years, mean \pm SD $12.7 \pm 3.8$ ) after radical surgical removal of craniopharyngioma (CF) with endosellar (ES; $n=32$ and suprasellar $(\mathrm{SS} ; \mathrm{n}=35$ ) location. Despite of severe $\mathrm{GH}$-deficiency (peak $\mathrm{GH}$ after clonidine-test $2.5 \mathrm{ng} / \mathrm{ml}$ ) $5 / 21$ children with ES CF and 10/19 with SS CF grew normally (growth velocity $5-12 \mathrm{~cm} /$ year), and growth velocity significantly correlated with area under curve insulin secretion after oral glucose load (INSAUC): $r=$ $0.352, p=0.008$. Insulin hypersecretion was accompanied by obesity: correlation between INSAUC and BMI (body mass index): $r=0.637, p<0.001$. Obesity and normal growth was more common for SS CF.

\begin{tabular}{lcll}
\hline Location & Height SDS & $\begin{array}{l}\text { Height velocity } \\
\text { cm/year }\end{array}$ & $\begin{array}{l}\mathrm{BMI} \\
\mathrm{kg} / \mathrm{m}^{2}\end{array}$ \\
\hline ES & $-1.9 \pm 1.6$ & $3.9 \pm 2.5$ & $17.4 \pm 2.1$ \\
SS & $-0.8 \pm 1.4$ & $5.3 \pm 2.7$ & $24.5 \pm 2.3$ \\
$\mathrm{P}$ & 0.02 & 0.1 & $<0.001$
\end{tabular}

Endocrine deficit was revealed more frequently in patients with ES CF, except for diabetes insipidus. Results:

\begin{tabular}{lllll}
\hline \multirow{2}{*}{ Location } & \multicolumn{4}{l}{ Patients with pituitary deficit } \\
\cline { 2 - 5 } & GH & TTH & ACTH & ADH \\
\hline ES & $30 / 30100 \%$ & $30 / 3294 \%$ & $27 / 3284 \%$ & $23 / 3272 \%$ \\
SS & $31 / 31100 \%$ & $29 / 3388 \%$ & $20 / 3361 \%$ & $27 / 3382 \%$ \\
\hline
\end{tabular}

In Conclusion: Postoperative examinations showed high incidence of hypopituitarism in children with CF, especially in ES location. Hyperinsulinemia may be involved in mechanism of postoperative weight gain and growth of children with CF. 
P3-563 Neuroendocrinology

Panhypopituitarism, Deficiency of Factors V, VIII, and vWf - an Uncommon Association

C.M. Tanaka*1;E.A. D'Amico*1; F.D.A. Carneiro*1;

V. Dichtchekenian*1; D. Damiani*1; N. Setian ${ }^{* 1} ;$ Introduced by

M.O. Savage

1 Pediatric Endocrinology Unit, Instituto da Criança, São Paulo

University Medical School and Hemotherapy Division, São Paulo, Brazil

Introduction: Combined multiple clotting deficiencies are rare and, when present, factor V and VIII is the commonest association. Although it is known that hypothyroid patients may have a decrease in von Willebrand's factor (vWf) and factor VIII, there have not been reports of panhypopituitarism associated with vWf and factor V and VIII deficiencies. Aim: To call attention to this unusual association. Case Report: This 9.6-year-old prepubertal boy, born to consanguineous parents, came because of short stature, microphallus $(1.2 \mathrm{~cm})$, and pallor. His $\mathrm{GH}$ peaked at $0.9 \mathrm{ng} / \mathrm{ml}, \mathrm{TSH}=0.6 \mathrm{mU} / 1$, free $\mathrm{T} 4=$ $0.2 \mathrm{ng} / \mathrm{dl}$. He had low TSH and PRL under TRH stimulation. Factor VIII $<1 \%$, Factor $\mathrm{V}=12 \%, \mathrm{vWf}=28 \%$. MRI revealed a partially empty sella, with pituitary hypoplasia. Treated with L-T4 he improved clinically and vWf increased to $68 \%$. At first, his pallor had been attributed to the bleeding disorder but, in fact, it was the result of hypothyroidism and disappeared upon treatment. Under hrGH he grew $12.7 \mathrm{~cm}$ in the first year (catch-up growth). It is noteworthy that this child has a 3-year-old brother with the same clotting defects, and his pituitary function is being checked. Discussion and Conclusion: As the genes responsible for pituitary development (pit 1, on the chromosome 3 ), vWf (on the 12), factor V (on the 1), and factor VIII (on the X) are on different chromosomes, it is difficult to suppose a common genetic defect explaining all the findings. It is more reasonable to suppose that this boy has a primary combined pituitary deficiency (Pit 1?) which, secondarily induces von Willebrand's disease and deficiency of factors V and VIII. To our knowledge, this association has not been reported in the medical literature.

P3-564 Autoimmunity, Syndromes and Ethics

\section{Endocrinopathies in Patients with Disorders of} Mitochondrial Energy Generating Metabolism

J. Kytnarová*1; L. Wenchich*1;H. Hansikova*1; J. Zeman ${ }^{*}$; Introduced by J. Lebl

${ }^{1}$ Charles University, Prague, Czech Republic

Inherited mitochondrial disorders of energy metabolism represent a large group of heterogeneous disorders, which may also manifest with different endocrinopathies. It is suggested that dysfunction of the respiratory chain complexes due to mutation in mitochondrial DNA may be found in 2-3\% of patients with diabetes mellitus type II. Not much is known about the prevalence of other endocrinopathies associated with disorders of mitochondrial energy generating systems. Objective: To evaluate the prevalence of endocrinopathies in patients with mitochondrial encephalomyopathies associated with dysfunction of respiratory chain and pyruvate dehydrogenase complexes. Patients and Methods: A retrospective study was performed in 53 patients in the age between 2 and 50 years with mitochondrial disorders of energy generating systems diagnosed in our department on enzymatic or molecular level. Cytochrome c oxidase (COX) deficiency was present in 26 patients, pyruvate dehydrogenase complex (PDH) deficiency in 5 patients, ATPase deficiency in 3 and deficiencies in other respiratory chain complexes in 4 patients. Investigation in mtDNA revealed MELAS syndrome in 5 patients, MERRF in 3 patients, Kearns-Sayre syndrome in 5 persons and Pearson syndrome in 1 patient. Results: Growth retardation (defined as height below - 2SDS) was observed in 25 patients (18 from 26 with COX deficiency, 1/5 with PDH deficiency, 2/5 persons with MELAS, 3/5 persons with Kearns-Sayre syndrome and 1/3 patients with ATPase deficiency). Growth retardation was the first symptom in 4 patients, in 2/26 with COX deficiency and 2/5 with Kearns-Sayre syndrome. In 1 child the growth hormon deficiency was proved (Kearns-Sayre syndrome). In 10 from 26 patients with COX deficiency intrauterine growth retardation was observed. Diabetes mellitus type 2 developed in 3 from 5 patients with MELAS syndrome (A3243G) and 1 from 5 patients with Kearns-Sayre syndrome. Hypothyreosis was found in one patient with MELAS. Hypertrichosis was observed in 8 from 26 patients with COX deficiency with Leigh syndrome and in 2 patients with PDHc deficiency. Conclusions: Endocrine symptoms may occur quite frequently in patients with mitochondrial encephalomyopathies. The mechanism of potential endocrine dysfunction remains unknown.

\section{P3-565 Autoimmunity, Syndromes and Ethics \\ Mutations in the COL11A1 Gene in a Family with Marshall Syndrome in Four Family Members}

D. Veimo*1; L. Tranebjaerg*2; Introduced by 0 . Trygstad

${ }^{1}$ Children's Department, Nordland Central Hospital, Bodoe, Norway; ${ }^{2}$ Department of Medical Genetics, University of Tromsoe, Tromsoe, Norway

The Marshall and Stickler syndromes are autosomal dominant disorders characterized by typical facial, ocular, articular, auditory and statural features. Because of the overlapping phenotypes there has been continous debate whether they are distinct entities or just different manifestations of the same syndrome. Stickler syndrome has been linked to the COL2A1 gene locus, but there has also been reported changes in the COL11A1 gene in one patient with this syndrome. There has now been identified at least 20 mutations in the two locuses, eight in the COL2A1 gene and 12 in the COL11A1 gene, and the data suggest that the phenotype can in part be predicted from the genotype. Genotypic-phenotypic comparisons have revealed an association between the Marshall syndrome phenotype and splicing mutations of $54 \mathrm{bp}$ exons in the Cterminal region of the COL11A1 gene. This gene is over $200 \mathrm{~kb}$ and consists of 68 exons, and codes for the $\alpha 1$ chain of collagen IX. Here is presented a family with four members with Marshall syndrome, the mother and all her three children, two boys and one girl. They all have the key features of the syndrome, with a hypochondroplasia-like short stature, midfacial hypoplasia and a depressed nasal bridge, a short nose with anteverted nostrils, high myopia, hearing loss, lowered auricles and a minor hypertelorism. Skeletal anomalies are also present, with a lacking widening of the spinal interpeduncular distance, frontal thickening of the calvarium in the mother, but no articular skeletal anomalies. The statural shortness is present from birth, as are the facial anomalies, and obviously the visual and auditory problems. None of them has, until now, presented retinal detachments, which is a more common feature of the Stickler syndrome. The oldest boy is tested for growth hormone deficiency, which he has not, but is now on trial with hGH-therapy for the last year. There has been found a novel mutation in the COL11A1 gene in all four members of this family, a mutation separate from other mutations found in the gene. There is a $\mathrm{C} \rightarrow \mathrm{A}$ substitution in position +3 e 50 , which has been proven to have an effect on the splicing of the nearby exons. 
P3-566 Autoimmunity, Syndromes and Ethics

\section{Kabuki Syndrome: Two Cases Presenting with} Endocrine Problems

A. Bereket ${ }^{* 1} ;$ S. Turan*2; Introduced by F. Darendeliler

${ }^{1}$ Marmara University, Istanbul, Turkey; ${ }^{2}$ Istanbul, Turkey

Kabuki syndrome (KS) is a mental retardation-malformation syndrome affecting multiple organ systems with a broad spectrum of abnormalities. Endocrine abnormalities are rarely seen in Kabuki syndrome. We present two new cases of KS from Turkey presenting with endocrine problems. Case 1: A 5-year-old male was referred for evaluation of history of hypoglycemia. History was remarkable for delivery by $\mathrm{C}$-section due to breech presentation at term, cleft palate detected at birth, recurrent episodes of respiratory problems and seizures starting at 2 months of age. Physical examination revealed long palpebral fissures with eversion of the lateral third of the lower eyelids, bilateral ptosis, blue sclerae, prominent ears, congenital alopecia areata, a systolic murmur, bilateral undescended testes and severe motor and mental retardation. He was found to have atrial septal defect, horseshoe kidney and cerebellar atrophy by imaging studies. Based on characteristic facial features, neuromotor retardation and other congenital abnormalities, diagnosis of KS was established. This patient had two documented episodes of severe hypoglycemia between 2.5 and 3.5 years of age. A fasting challenge did not reveal underlying etiology for hypoglycemia which did not recur after 3.5 years of age. Case 2: A 16-month-old girl was referred for evaluation of breast enlargement. History was remarkable for delivery by C-section, neonatal polycythemia and developmental delay. The breast development was first noticed at 11 months of age. Physical examination revealed typical facial features as in patient 1 , in addition to high arched palate, widely spaced teeth, a hyperpigmented keratotic lesion at lumbar region and bilateral breast enlargement (Tanner III). There was no pubic or axillary hair, excessive growth or estrogen effect on vaginal mucosa. The bone age was 18 months. Baseline FSH, LH and Estradiol concentrations were normal. She was found to have hydronephrosis and bilateral vesicoureteral reflux during workup for urinary tract infection. Diagnosis of KS was established based on facial features, developmental delay and urinary tract abnormalities. A follow-up examination 4 months later did not show any progression or regression of the breast enlargement consistent with benign premature thelarche. Although premature thelarche has been described in $23 \%$ of girls with KS, hypoglycemia has been reported in a single case previously. Patient 2 represents the second case in the literature with neonatal polycythemia. Cerebellar atrophy, congenital scalp defect and keratotic skin lesion observed in our patients are features not previously reported in $\mathrm{KS}$.

P3-567 Autoimmunity, Syndromes and Ethics

\section{Phenotype-Genotype Correlation in Slovenian Patients with Autoimmune Polyglandular Syndrome Type I (APS-I) \\ T. Battelino ${ }^{1} ;$ K. Trebusak*1; N. Bratanic ${ }^{* 1} ;$ B. Repic-Lampret ${ }^{* 1}$. C. Krzisnik ${ }^{1}$ \\ ${ }^{1}$ University Children's Hospital, Ljubljana, Slovenia}

Autoimmune polyglandular syndrome type 1 (APS-I) is a rare autosomal recessive disease. It is characterised by presence of two of the three major clinical symptoms: hypoparathyroidism, Addison's disease and mucocutaneous candidiasis. Several other disorders may be present, such as ectodemal dystrophy, gonadal failure, IDDM, hypothyroidism. The disease usually occurs in childhood, but new tissue-specific symptoms may appear throughout life. The aetiology of APS-I is associated with mutations in the AIRE (autoimmune regulator) gene. Objective: To determine phenotype-genotype correlation in 5 APS-I patients from 4 unrelated Slovenian families. Patients and Methods: Clinical characteristics are summarised in the table.

\begin{tabular}{llllllllll}
\hline \multirow{2}{*}{ ID } & \multirow{2}{*}{ Sex } & YOB & \multicolumn{6}{c}{ Age at the outset of the symptom (y) } \\
\cline { 3 - 8 } & & & HP & Add & MCC & AI & ED & HA \\
\hline 1 A & m & 1979 & 14 & 10 & - & - & - & - \\
$1 \mathrm{~B}$ & $\mathrm{~m}$ & 1980 & 7 & - & 18 & 7.5 & - & - \\
2 & $\mathrm{f}$ & 1990 & 8 & 8 & - & - & - & - \\
3 & $\mathrm{~m}$ & 1990 & 6.5 & 6.5 & 6.5 & - & 6.5 & - \\
4 & $\mathrm{~m}$ & 1986 & 11 & 13 & 11 & - & - & 11 \\
\hline
\end{tabular}

HP = Hypoparathyroidism; Add = Addison's diasease MCC = mucocutaneous candidiasis; $\mathrm{Al}=$ alopecia; $\mathrm{ED}=$ ectodermal dystrophy; $\mathrm{HA}=$ hypoaldosteronism.

Exons of the AIRE gene were individually amplified with PCR and directly sequenced. Sequences were compared to the GeneBank Access. No. AB006684. Results: All patients were homozygous for mutation R257X in exon 6, which was confirmed by TaqI restriction digestion and FRLP analysis. Conclusion: Although all patients had the so called Finnish mutation which causes a truncated protein, the clinical presentation varied markedly from patient to patient, even between the bothers from the family 1 . No phenotypegenotype correlation was found. In contrast to the Finnish cohort of APS-I patients, ED was observed only in one patient. Other genetic and/or environmental factors must influence the phenotype and disease progression of individual APS-I patients.

\section{P3-568 Autoimmunity, Syndromes and Ethics \\ Informed Consent for Human Genetics Research \\ L.L. McCabe*1;W.W. Grody*1;B. Henker*1;C. Jaenicke*1; \\ S. Peckman ${ }^{* 1} ;$ R.S. Sparkes ${ }^{* 1} ;$ F. Wiley*1; E.R.B. McCabe ${ }^{* 1}$; \\ Introduced by R.G. Rosenfeld \\ ${ }^{1}$ UCLA School of Medicine, Los Angeles, CA, United States}

Objective: Our objective was to provide guidance for clinical investigators in the use of human samples for genetic research. Background: Genetic investigation involves all areas of clinical research, since genetic information may be quite sensitive, and is contained in sources as diverse as family histories and pathological specimens. Informed consent for human genetic research involves complex issues for the research subjects and the investigators. These issues include the nature of genetic information, privacy and confidentiality of genetic information, sharing tissue and/or information with other investigators, feedback to research participants, and sample ownership. Design and Methods: The Executive Vice Chancellor for UCLA created a Subcommittee of the Human Research Policy Board to develop a consistent and complete approach to prospective human tissue research. Over a six month period, the Subcommittee met to discuss published reports and guidelines from professional organizations on genetic testing, and to develop an appropriate informed consent policy to cover genetic research on human material. Results: The Subcommittee included standard language in the Consent Form to explain the information contained in DNA, RNA and protein. No relative would be contacted without the research subject's permission. If there is tissue left over at the end of the study, research subjects can specify on a checklist if they would like to have this material shared with other researchers or not. Participants can indicate their preference on a checklist to receive information about their sample, general information about the study results, or no information. In signing the Consent Form, subjects acknowledge that the University of California, or its designee, owns their sample. Conclusions: The Subcommittee prepared standard language for Informed Consent Forms for use with human tissue. UCLA investigators undertaking such research are asked to incorporate this language into their protocols, resulting in a more informative and consistent consent process. The work of the Human Subjects Institutional Reviews Boards has been enhanced by this unified approach to genetic research. Investigators who are not geneticists have been sensitized to the issues of genetic testing of tissue samples. While the Subcommittee recognized that issues of genetic testing are evolving, the standard language was an attempt to deal with current issues and to anticipate future concerns. 
P3-569 Autoimmunity, Syndromes and Ethics

Molecular Findings in a 6,8-Year-Old Turkish Boy with

\section{Triple A Syndrome}

K. Schmittmann-Ohters ${ }^{* 1} ;$ A. Huebner ${ }^{* 2}$; A. Richter-Unruh ${ }^{* 1}$; B.P. Hauffa ${ }^{1}$

${ }^{1}$ University Hospital Essen, Essen, Germany; ${ }^{2}$ University Childrens

Hospital Dresden, Dresden, Germany

The triple A syndrome, encompassing adrenal insufficiency, achalasia and alacrima, is caused by a yet unknown molecular defect in the region of chromosome $12 \mathrm{q} 13$. Associated features like neurological and dermatological symptoms are often seen with a large variability. Here we report a 6,8-year-old boy, who was born as the 13 th child of healthy, related parents from Turkey after an uncomplicated twin pregnancy. At the age of one year the twin brother died after a routine vaccination. Another brother died unexpectedly, when he was 3 years old. During his first year the boy presented with relapsing vomiting and failure to thrive. At the age of 4 years the diagnosis of congenital adrenal hyperplasia was made in Turkey, and therapy with hydrocortisone and fludrocortisone was initiated. The boy was 6,7 years old when he was hospitalized during a visit in Germany for repeated postprandial vomiting. Here he was diagnosed as having achalasia and primary adrenocortical hypofunction. Additionally, history and clinical examination (Schirmer's test) showed that the boy was unable to produce tears (alacrima). Thus, the clinical diagnosis of triple A syndrome was made. Molecular analysis showed homozygosity of 10 chromosome 12q13 markers (D12S1629-D12S1707), supporting a defect in the triple A region. One male sibling was also examinated and proved to be a heterozygous carrier of the disease. Examination of the parents will follow. Molecular genetic studies in families with triple A syndrome are needed to elucidate the nature of the underlying gene defect. 